\title{
Electrochemical detection of fentanyl using screen-printed carbon electrodes with confirmatory analysis of fentanyl and its analogs in oral fluid using liquid chromatography-tandem mass spectrometry
}

Colby E. Ott

West Virginia University, ceo0009@mix.wvu.edu

Follow this and additional works at: https://researchrepository.wvu.edu/etd

Part of the Analytical Chemistry Commons, and the Toxicology Commons

\section{Recommended Citation}

Ott, Colby E., "Electrochemical detection of fentanyl using screen-printed carbon electrodes with confirmatory analysis of fentanyl and its analogs in oral fluid using liquid chromatography-tandem mass spectrometry" (2019). Graduate Theses, Dissertations, and Problem Reports. 7470.

https://researchrepository.wvu.edu/etd/7470

This Thesis is protected by copyright and/or related rights. It has been brought to you by the The Research Repository @ WVU with permission from the rights-holder(s). You are free to use this Thesis in any way that is permitted by the copyright and related rights legislation that applies to your use. For other uses you must obtain permission from the rights-holder(s) directly, unless additional rights are indicated by a Creative Commons license in the record and/ or on the work itself. This Thesis has been accepted for inclusion in WVU Graduate Theses, Dissertations, and Problem Reports collection by an authorized administrator of The Research Repository @ WVU. For more information, please contact researchrepository@mail.wvu.edu. 
Electrochemical detection of fentanyl using screen-printed carbon electrodes with confirmatory analysis of fentanyl and its analogs in oral fluid using liquid chromatography-tandem mass spectrometry

Colby Ott

Thesis submitted

to the Eberly College of Arts and Sciences at West Virginia University

in partial fulfillment of the requirements for the degree of

Master of Science in

Forensic and Investigative Science

Luis Arroyo, Ph.D., Chair

Tatiana Trejos, Ph.D.

Nianqiang Wu, Ph.D.

John Richardson, Ph.D.

Department of Forensic and Investigative Science

Morgantown, West Virginia

2019

Keywords:

Electrochemistry, Screen-printed electrodes, Fentanyl, Tandem Mass Spectrometry, Forensic Science, Novel Psychoactive Substances

Copyright 2019 Colby E. Ott 


\begin{abstract}
Electrochemical detection of fentanyl using screen-printed carbon electrodes with confirmatory analysis of fentanyl and its analogs in oral fluid using liquid chromatography-tandem mass spectrometry

Colby Ott
\end{abstract}

Utilizing screen-printed carbon electrodes (SPCEs), a fast, simple, and sensitive approach toward the detection, identification, and quasi-quantitation of fentanyl was achieved both in an electrochemical cell and as a drop on the electrode surface. Electro-oxidation of fentanyl at the electrode was demonstrated using adsorptive stripping square-wave voltammetry between $-0.5 \mathrm{~V}$ and $+1.6 \mathrm{~V}$ with $100 \mathrm{mM}$ Tris- $\mathrm{HCl}$ buffer at $\mathrm{pH} 8.5$ as supporting electrolyte. Parameter optimization was conducted during method development to include supporting electrolyte and $\mathrm{pH}$, electrochemical technique, pre-treatment and equilibration time, and various surface modifications. The simplest method utilizing an unmodified SPCE was determined to be appropriate for the identification of fentanyl. Electro-oxidation of the fentanyl compound was observed to occur as an irreversible process due to both diffusion to the electrode surface and oxidation of adsorbed species on the working electrode.

The resulting voltammograms demonstrated the presence of two oxidation peaks at 750 $\mathrm{mV}$ (peak I) and $880 \mathrm{mV}$ (peak II) versus a pseudo- $\mathrm{Ag} / \mathrm{AgCl}$ reference. Fentanyl oxidation was observed at concentrations of $\sim 76 \mathrm{ng} / \mathrm{mL}$ in cell and $\sim 300 \mathrm{ng} / \mathrm{mL}$ in a $100 \mu \mathrm{L}$ drop. Statistical limits of detection were determined to be slightly above the observable oxidation peaks with limits of detection of $145 \mathrm{ng} / \mathrm{mL}$ for the cell method and $530 \mathrm{ng} / \mathrm{mL}$ for the drop method. Reproducibility between electrodes, assessed as the average relative standard deviation (RSD), for peak I and peak II in the cell was $12 \%$ and $18 \%$, respectively. RSD in the drop was $13 \%$ and $15 \%$ for peaks I and II.

Accuracy of the detection method was determined in the cell by analyzing single-blind samples prepared in the laboratory and demonstrated better accuracy in lower concentrations of fentanyl versus higher concentrations. The effects of interfering compounds were considered due to the likelihood of fentanyl being found in mixtures. Quinine and cocaine were found to interfere with peak II, while peak I remained identifiable except when present with large concentrations of 
interferent. Methamphetamine was observed to have a similar effect although drastically reduced in comparison to both quinine and cocaine. Acetaminophen and caffeine did not produce interfering signals. Analysis at various ratios of the compounds demonstrated that the identification of fentanyl could still be achieved through the presence of peak I. The oxidative mechanism of fentanyl was proposed based on the literature available for the oxidation of amines and voltammetric data present for fentanyl and related compounds. The proposed mechanism rejects some previously hypothesized oxidation mechanisms of tertiary amines where the presence of two peaks was observed. It was suggested that a two-step oxidation process of the tertiary amine followed by the oxidation of the newly formed secondary amine product resulted in the two observable peaks. However, this work agrees with literature supporting the effect of adsorption of the tertiary diamine to the electrode surface. This mechanism is presented herein, whereby the observed oxidation peaks result from the adsorbed species and the diffusion of the species to the electrode surface, owing to the difference in peak potentials for peak I and peak II.

A confirmatory LC/MS/MS method for the analysis of fentanyl and fentanyl analogs in oral fluid was developed and validated. Optimization of fragmentor voltage, collision energy, and fragmentation ions was achieved and used in the construction of a dynamic multiple reaction monitoring (dMRM) method. Chromatographic separation demonstrated resolution between 13 fentanyl-related compounds along with 7 internal standards. The calibration model used was linear with a weighting of $1 / \mathrm{x}$ between the range of $0.1 \mathrm{ng} / \mathrm{mL}$ to $50 \mathrm{ng} / \mathrm{mL}$. The limit of detection for the majority of drugs was determined to be $0.01 \mathrm{ng} / \mathrm{mL}$ with the limit of quantitation at the lowest calibrator of $0.1 \mathrm{ng} / \mathrm{mL}$ with correlation coefficients between 0.9992-0.9999. Bias, precision, matrix effects, recovery, and process efficiency were assessed and were within the guideline range for acceptability for the majority of analytes assessed using a solid-phase extraction procedure with spiked oral fluid. Twelve commonly encountered illicit drugs were used to assess selectivity. No interferences were found for fentanyl or its analogs. Stability was assessed for processed samples kept at room temperature in auto-sampler and the freezer, as well as, for freeze/thaw stability. The majority of analytes were considered stable under all conditions for up to 72 hours.

Together these two methods demonstrate the identification and quasi-quantitation of fentanyl through electrochemical oxidation and confirmatory analysis via liquid chromatographytandem mass spectrometry (LC/MS/MS). The combined use of these techniques seeks to emulate 
the SWGDRUG requirement, although electrochemistry has, to this point, not been included in the list of acceptable techniques.

Other work contained herein demonstrates assessment of various electrode modification techniques to improve the signal of fentanyl, attempts at enzymatic detection of codeine and fentanyl utilizing cytochrome P450 isozymes 2D6 and 3A4, and electrochemical detection of the synthetic cannabinoid PB-22. 


\section{ACKNOWLEDGEMENTS}

There have been many people throughout my life who have made a great impact on myself and on my education to whom I owe many thanks. Without these many people, this work and my own success would not have been possible.

First, I must thank my research advisor Dr. Luis Arroyo. His constant support, motivation, advice, and belief in me have allowed for the completion of this work and for my growth as an individual, researcher, and professional. His confidence in me, along with the knowledge I have gained from him, has led me to become a more independent and analytical thinker. Dr. Arroyo has continued to push me to be the best that I can be while providing me with opportunities I would not have had except for his help and mentorship. This mentoring and friendship have enabled both this research, the growth of our research group, and our deep respect for him.

I also must thank my committee. Their combined knowledge and experience has been an invaluable tool and resource toward the completion of this work and increasing my own knowledge and expertise. Their teaching has enhanced my experience in research and in the classroom and has given me insights into the many intricacies involved in research.

My research group and classmates are owed thanks for their support of the research project and of me. They have served as a valuable resource for figuring out problems, bouncing ideas off of, and hearing opinions other than my own. Of these people, I owe a special thanks to Joseph Cox whose knowledge and opinion has meant a great deal to me. The ability to discuss various problems, projects, and questions has allowed for success, friendship, and helped me find what I am passionate about.

I would also like to extend my appreciation to our colleagues in Spain, Dr. Julia ArcosMartínez and Dr. Hugo Cunha-Silva. Their support of both me and this project is greatly appreciated, and without which this project would have been much different. Their knowledge of electrochemistry has been an excellent teaching tool, helping me to become more skilled in the field and in research. Hugo's support and friendship have been invaluable, and both he and Dr. Arcos-Martínez have served as mentors to whom I greatly admire.

Of course, this work would be absent except for the many great teachers and professors of which I have had the pleasure of being in their classroom from my days in elementary to my time as a graduate student. Their love of learning, teaching, and for their students has instilled in me a drive for discovering new things and the will to never stop learning and growing. 
Lastly, I must thank my family for their tremendous support and encouragement throughout my life. Without their kindness, compassion, love, and support my journey through life would be much different. Family has always been a source of support and strength for me. I also owe a huge thank you to one of my biggest supporters and influences in my life: my grandma Ann. Her love of science, nature, and learning new things sparked that same love in me. From the time I was little, she always nurtured and helped grow the scientist living inside me and drove me to always do my best in school. Her love and support have been constant and always growing, serving as inspiration and motivation to pursue my dreams. Finally, thank you to my parents who have always been there for me no matter the need. Without their love and their selfless support, I could never have achieved all that I have. I am extremely lucky to have them as parents. Thank you for everything you do to bless and enrich my life.

Thank you all! 
Dedicated to the memory of my great-grandparents

Nanny and Pappy Miller and to my Grandma Pearl 


\section{TABLE OF CONTENTS}

List of Figures and Tables .......................................................................................xii

List of Abbreviations.............................................................................................xvi

Part I: Introduction........................................................1

Chapter 1: Purpose of Research and Objectives.....................................................1

1.1 - Problem Statement and Purpose of the Study ................................................

1.2 - Goals and Objectives..................................................................................

Part II: Electrochemical Analysis of Fentanyl.................................8

Chapter 1: Relevant Literature Review....................................................................................8

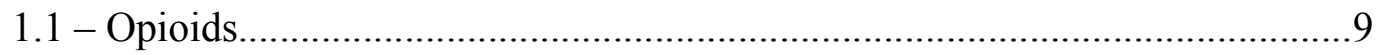

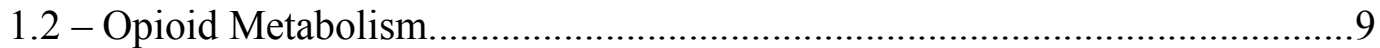

1.3 - Fentanyl and Fentanyl Analogs............................................................ 10

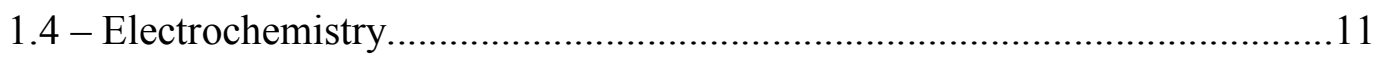

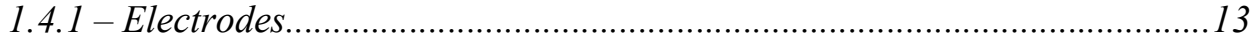

1.4.2 - Voltammetric Techniques...................................................................... 14

1.4.3 - Amperometric Techniques..................................................................17

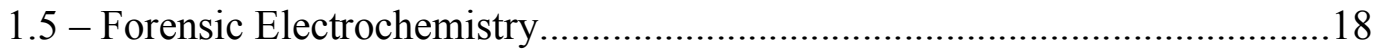

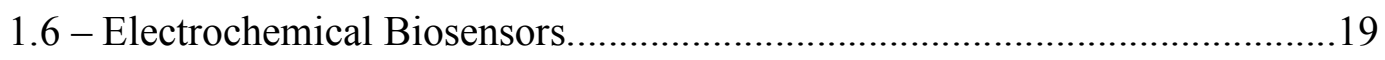

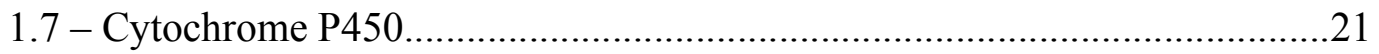

1.8 - Nanomaterials and Electrode Modifications..........................................24

1.9 - Method Validation for Qualitative Identification and Screening.............26

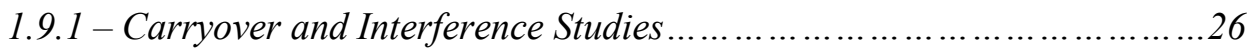

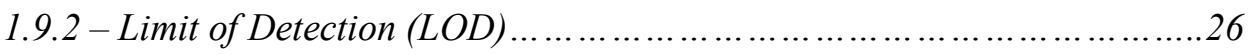

Chapter 2: Materials, Methods, and Experimental Design..............................................28

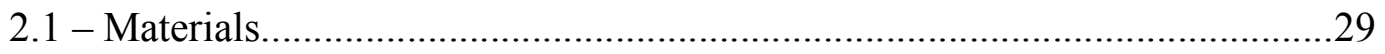

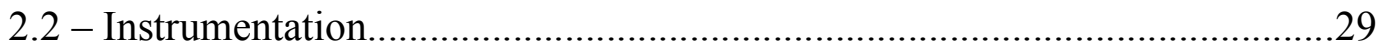


2.3 - Preparation of Standard Working Solution............................29

2.4 - Manufacture of Screen-Printed Carbon Electrodes.................................29

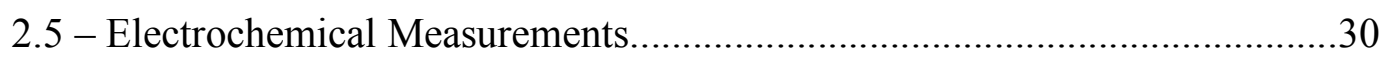

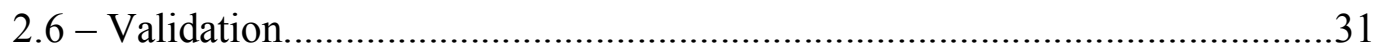

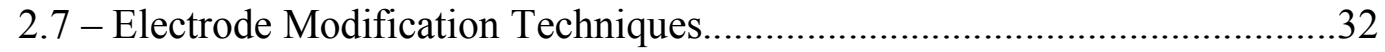

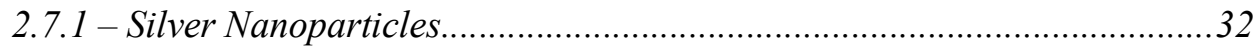

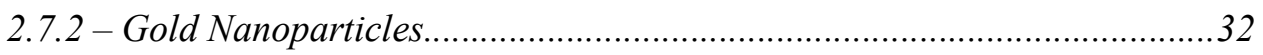

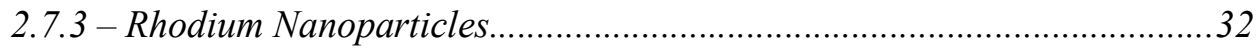

2.7.4 - Multiwalled Carbon Nanotubes..................................................................33

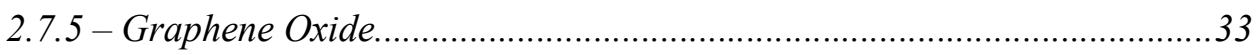

Chapter 3: Results and Discussion...................................................................... 34

3.1 - Electrochemical Behavior of Fentanyl..................................................35

3.2 - Optimization of Parameters and Techniques............................................36

3.3 - Electrochemical Detection of Fentanyl..............................................40

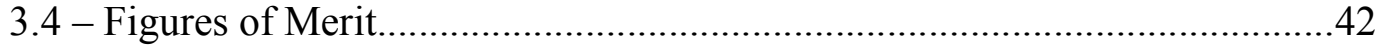

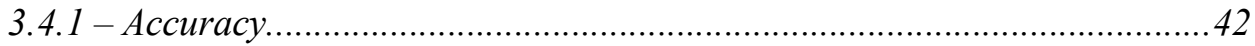

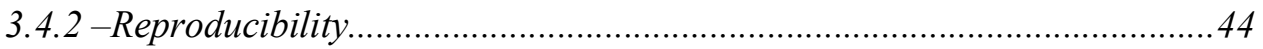

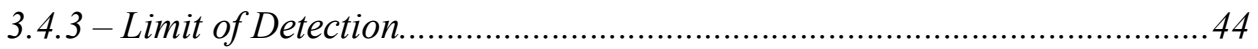

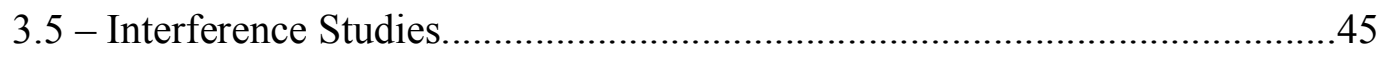

3.6 - Hypothesized Redox Mechanism...........................................................51

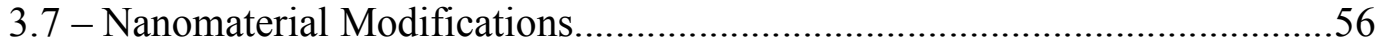

3.8 - Anodic Stripping Square-Wave Voltammetry......................................61

Chapter 4: Conclusions and Future Work............................................................6 63

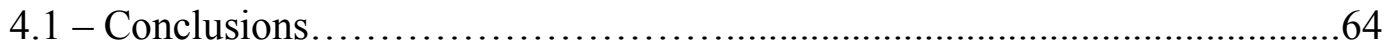

4.1 - Future Work...................................................................65

Part III: LC/MS/MS Analysis of Fentanyl and its Analogs in Oral Fluid...67

Chapter 1: Relevant Literature Review..........................................................67

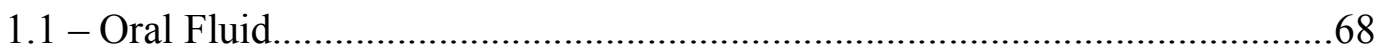


1.2 - Method Validation: LC/MS.......................................................................

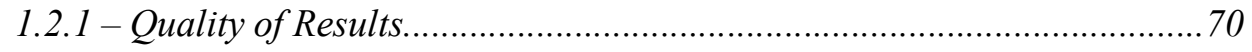

1.2.2 - Calibration Model and Carryover........................................................ 71

1.2.3 - Interference/Selectivity Studies.............................................................71

1.2.4 - Recover, Matrix Effects, and Process Efficiency..................................72

1.2.5 - Limit of Detection and Lowest Limit of Quantitation..............................73

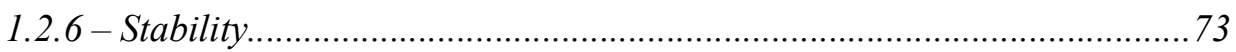

Chapter 2: Materials, Methods, and Experimental Design.............................................75

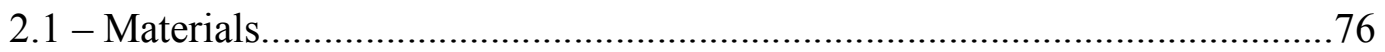

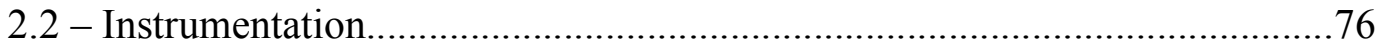

2.3 - Preparation of Standard Working Solutions................................................

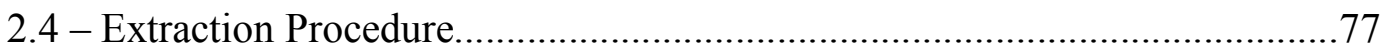

2.5 - Liquid Chromatography..............................................................................

2.6 - Mass Spectrometry................................................................................

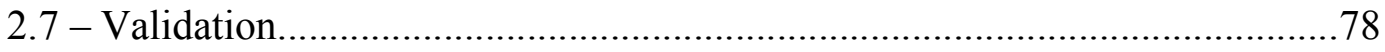

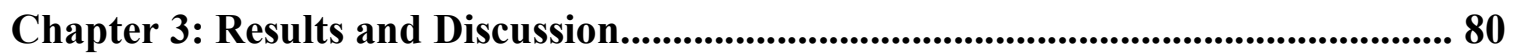

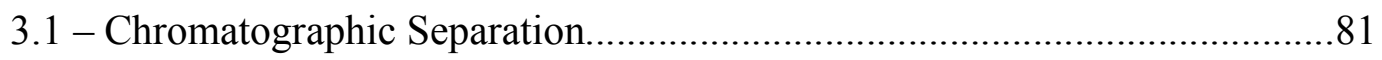

3.2 - LC/MS/MS Method Validation.................................................................

Chapter 4: Conclusions and Future Work.......................................................................... 89

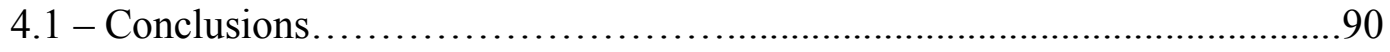

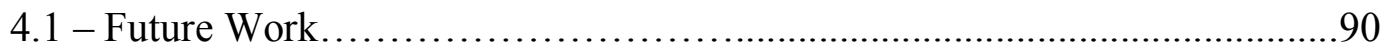

Part IV: Overall Conclusions................................................91

Chapter 1: Overall Concluisions..............................................................................................91

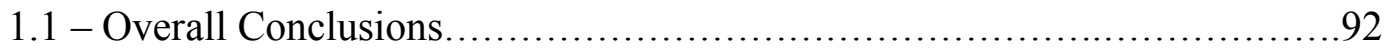

Part V: Other Electrochemical Methods.....................................93

Chapter 1: Other Methods..........................................................................................................93 
1.1 - Enzymatic Biosensor for Codeine: Preparation for Fentanyl...................94

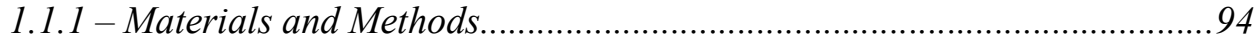

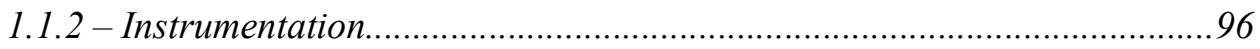

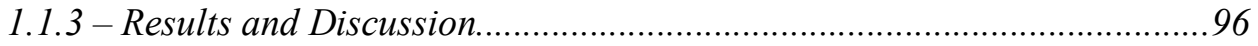

1.2 - Enzymatic Biosensor for the Detection of Fentanyl.................................98

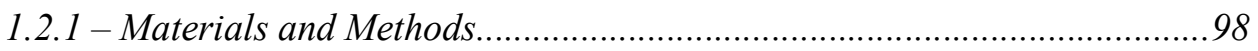

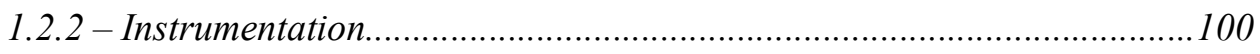

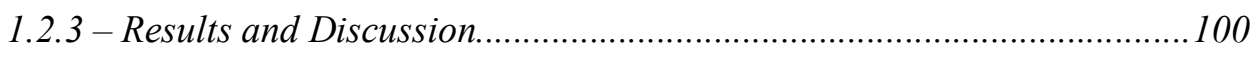

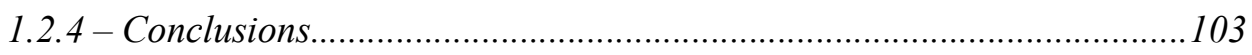

1.3 - Detection of the Synthetic Cannabinoid PB-22 ........................................ 103

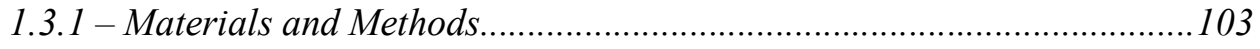

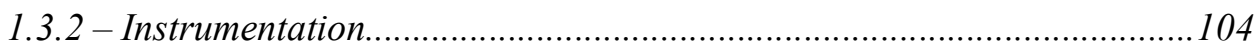

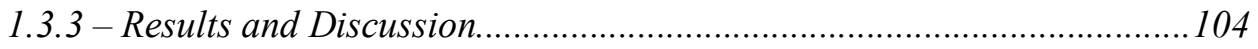

1.3 .4 -Conclusions ...................................................... 106

References.............................................................................................107

Appendix 


\section{LIST OF FIGURES AND TABLES}

\section{Figures:}

Figure 1: $\quad$ Potential modification sites of fentanyl

Figure 2: $\quad$ Fentanyl structure demonstrating scaffold regions as locations for modifications

Figure 3: $\quad$ Cell set-up utilizing conventional electrodes with an approximate height of $15 \mathrm{~cm}$ (left) and screenprinted carbon electrodes used in this work with an approximate size of $3.5 \mathrm{~cm} \times 1.5 \mathrm{~cm}$ demonstrating use in cell or with a drop (right).

Figure 4: $\quad$ Cyclic voltammogram for fentanyl at $1.46 \mathrm{mM}$ (left) and waveform demonstrating the cyclic nature of the potential applied (right).

Figure 5: $\quad$ Adsorptive stripping square-wave voltammogram for fentanyl at $3.62 \mu \mathrm{g} / \mathrm{mL}$ (left) and waveform demonstrating a pulsed signal superimposed on a staircase giving the forward and reverse waves (right).

Figure 6: $\quad$ Heme (iron protoporphyrin IX) group

Figure 7: $\quad$ Catalytic cycle of a generic CYP enzyme

Figure 8: $\quad$ CYP2D6 enzyme ribbon diagram

Figure 9: $\quad$ CYP3A4 enzyme ribbon diagram

Figure 10: $\quad$ Cyclic voltammetry of fentanyl at a SPCE in a $5 \mathrm{~mL}$ cell containing PBS buffer $\mathrm{pH} 7.4$ (left) and resulting calibration curve for $\mathrm{CV}$ performed in PBS supplemented with chloride.

Figure 11: Cyclic voltammetry of citrate in PBS buffer supplemented with chloride and nitrate demonstrating no response to citrate and response to only fentanyl.

Figure 12: $\quad$ Current response to $151 \mu \mathrm{g} / \mathrm{mL}$ fentanyl at differing $\mathrm{pH}$ values. Three replicates were performed per $\mathrm{pH}$ value. The analysis was performed in a drop on the SPCE surface.

Figure 13: Demonstration of peak improvement based on buffer $\mathrm{pH}$ showing improved peak current and twopeak definition at a $\mathrm{pH}$ of 8.5 in PBS.

Figure 14: Comparison of calibration curves for SWV (left) and DPV (right) methods demonstrating higher sensitivity with SWV.

Figure 15: $\quad$ Stirred equilibration time optimization in a cell containing Tris- $\mathrm{HCl}$ at a fentanyl concentration of $336 \mathrm{ng} / \mathrm{mL}$. Three replicates per time were tested with an RSD of approximately $9 \%$ at 320 seconds (left) and stirred equilibration time in $100 \mu \mathrm{L}$ drop of Tris- $\mathrm{HCl}$ at $336 \mathrm{ng} / \mathrm{mL}$ with RSD of approximately $4.5 \%$ for two replicates at $\mathrm{pH} 8.5$ (right).

Figure 16: $\quad$ AdS-SWV for the standard addition of fentanyl to a $5 \mathrm{~mL}$ electrochemical cell containing $100 \mathrm{mM}$ Tris-HCl pH 8.5 in triplicate (left) and constructed calibration curve for peaks I and II for fentanyl (right).

Figure 17: $\quad$ AdS-SWV for increasing concentrations of fentanyl in a $100 \mu \mathrm{L}$ drop of Tris- $\mathrm{HCl}$ on the surface of the SPCE (left) and constructed calibration curve for fentanyl in drop assessed in triplicate (right).

Figure 18: $\quad$ Adsorptive stripping square-wave voltammograms for the standard addition of methamphetamine (left) and caffeine (right) in a $5 \mathrm{~mL}$ electrochemical cell containing $100 \mathrm{mM}$ Tris- $\mathrm{HCl} \mathrm{pH} \mathrm{8.5.}$ 
Figure 19: $\quad$ Adsorptive stripping square-wave voltammograms obtained in a $5 \mathrm{~mL}$ cell with $100 \mathrm{mM}$ Tris-HCl $\mathrm{pH} 8.5$ for the analysis of fentanyl in the presence of methamphetamine at ratios of 0.3:10 (left), 0.3:20 (middle), and 1:10 (right), respectively.

Figure 20: $\quad$ Adsorptive stripping square-wave voltammograms for the standard addition of cocaine in a $5 \mathrm{~mL}$ electrochemical cell containing $100 \mathrm{mM}$ Tris- $\mathrm{HCl} \mathrm{pH} 8.5$ and the corresponding standard curve.

Figure 21: $\quad$ Adsorptive stripping square-wave voltammograms in a $5 \mathrm{~mL}$ cell with $100 \mathrm{mM}$ Tris- $\mathrm{HCl} \mathrm{pH} 8.5$ for the analysis of fentanyl in the presence of cocaine at ratios of 0.3:10 (left), 1:10 (middle), and 1:3 (right), respectively.

Figure 22: $\quad$ Adsorptive stripping square-wave voltammograms for the standard addition of quinine in a $5 \mathrm{~mL}$ electrochemical cell containing $100 \mathrm{mM}$ Tris- $\mathrm{HCl}$ pH 8.5 and corresponding calibration curve.

Figure 23: $\quad$ Adsorptive stripping square-wave voltammograms in a $5 \mathrm{~mL}$ cell with $100 \mathrm{mM}$ Tris- $\mathrm{HCl} \mathrm{pH} 8.5$ for the analysis of fentanyl in the presence of quinine at ratios of 0.3:10 (left), 1:10 (middle), and 1:3 (right), respectively.

Figure 24: Adsorptive stripping square-wave voltammograms for the standard addition of acetaminophen in a $5 \mathrm{~mL}$ electrochemical cell containing $100 \mathrm{mM}$ Tris- $\mathrm{HCl} \mathrm{pH} 8.5$ and corresponding calibration curve.

Figure 25: $\quad$ Adsorptive stripping square-wave voltammograms in a $5 \mathrm{~mL}$ cell with $100 \mathrm{mM}$ Tris- $\mathrm{HCl} \mathrm{pH} 8.5$ for the analysis of fentanyl in the presence of acetaminophen at ratios of 0.3:10 (left), 1:10 (middle), and 1:3 (right), respectively.

Figure 26: Cyclic voltammograms collected at varying scan rates (right) to demonstrate that the electrooxidation of fentanyl involves both diffusion (top left) and adsorption (bottom left).

Figure 27: $\quad$ Successive cyclic voltammograms on a $100 \mu \mathrm{L}$ drop of $1 \mathrm{mM}$ fentanyl in Tris-HCl $\mathrm{pH} 8.5$ demonstrating the irreversible electron transfer process.

Figure 28: $\quad$ Proposed mechanism for the electrooxidation of fentanyl and rejected mechanism

Figure 29: $\quad$ LC/MS/MS results following the electrochemical analysis of fentanyl demonstrating the increasing concentration of norfentanyl, supporting the proposed oxidation pathway.

Figure 30: $\quad$ AdS-SWV for increasing concentrations of fentanyl in Tris- $\mathrm{HCl}$ at a modified $\mathrm{SPC}_{\mathrm{Rhnps}} \mathrm{E}$ (left) and constructed calibration curve for fentanyl (right).

Figure 31: $\quad$ SWV for increasing concentrations of fentanyl in Tris- $\mathrm{HCl}$ at a modified $\mathrm{SPC}_{\mathrm{MWCNT}}$ (left) and constructed calibration curve for fentanyl (right).

Figure 32: $\quad$ SWV for increasing concentrations of fentanyl in Tris- $\mathrm{HCl}$ at a modified $\mathrm{SPC}_{\mathrm{rGO}} \mathrm{E}$ (left) and constructed calibration curve for fentanyl (right).

Figure 33: $\quad \mathrm{SWV}$ for increasing concentrations of fentanyl in Tris- $\mathrm{HCl}$ at a modified $\mathrm{SPC}_{\mathrm{Agnps}} \mathrm{E}$ (left) and constructed calibration curve for fentanyl (right).

Figure 34: $\quad$ SWV for increasing concentrations of fentanyl in Tris- $\mathrm{HCl}$ at a modified $\mathrm{SPC}_{\mathrm{Agnps} / \mathrm{MWCNTE}}$ (left) and constructed calibration curve for fentanyl (right).

Figure 35: $\quad$ Chromatographic separation of analytes of interest using dMRM.

Figure 36: Chromatographic separation of 25 drugs of abuse, including the 13 target analytes. 
Figure 37: Graphical depiction of the stability of all analytes over 72 hours for freeze/thaw (a), autosampler at room temperature (b), and freezer at $-20^{\circ} \mathrm{C}(\mathrm{c})$.

Figure 38: Covalent attachment strategy via self-assembled monolayer for CYP2D6 on the carbon surface of SPCE.

Figure 39: Attachment to the diazonium salt to the working electrode surface (left) and reduction of the nitro groups to amine groups(right).

Figure 40: Cyclic voltammograms in PBS buffer demonstrating the difference between the blank SPCE and the enzyme modified SPCE.

Figure 41: Chronoamperogram for the standard addition of $20 \mu \mathrm{L}$ of $30 \mu \mathrm{g} / \mathrm{mL}$ codeine to a $150 \mu \mathrm{L}$ drop of phosphate buffer (left) and resulting calibration curve (right).

Figure 42: $\quad$ Immobilization strategy via self-assembled monolayer for CYP3A4 on the gold surface of $\mathrm{SP}_{\mathrm{Au}} \mathrm{E}$.

Figure 43: $\quad$ Constructed chronoamperograms for fentanyl detection at a $\mathrm{SP}_{\mathrm{Au}} \mathrm{E}$ in a $100 \mathrm{uL}$ drop of $50 \mathrm{mM}$ PBS supplemented with $100 \mathrm{mM} \mathrm{NaNO}_{3}$ (left) and calibration curve (n=4) with RSD of $17 \%$.

Figure 44: $\quad$ SEM-EDS spectra for a bare $\mathrm{SP}_{\mathrm{Au}} \mathrm{E}$ (left), MPS modified $\mathrm{SP}_{\mathrm{Au} / \mathrm{MPS}} \mathrm{E}$ (middle), and PDDA modified $\mathrm{SP}_{\mathrm{Au} / \mathrm{MPS} / \mathrm{PDDA}} \mathrm{E}$ (right).

Figure 45: $\quad$ SEM images for the blank $\mathrm{SP}_{\mathrm{Au}} \mathrm{E}$ ( $\mathrm{a}$ and $\mathrm{b}$ ), the MPS modified electrode (c), and the MPS/PDDA modified electrode (d).

Figure 46: $\quad$ AFM images plotting the height of the blank $\mathrm{SP}_{\mathrm{Au}} \mathrm{E}$ (a), MPS modified electrode (b), and MPS/PDDA modified electrode (c).

Figure 47: Cyclic voltammogram in $0.5 \mathrm{M}$ sulfuric acid for electrochemical cleaning the platinum working electrode.

Figure 48: $\quad$ Differential pulse voltammograms for analysis of PB-22 in the cell (left) and the resulting calibration curve for 3 replicates (right).

\section{List of Tables}

Table 1: $\quad$ Literature review of fentanyl detection via electrochemical methods

Table 2: $\quad$ Relative standard deviation, as percent, for triplicate analysis of the $\mathrm{pH}$ range

Table 3: $\quad$ Accuracy of single-blind test samples containing fentanyl

Table 4: $\quad$ Single-blind test sample analysis

Table 5: $\quad$ Comparison between electrode modifications/detection methods

Table 6: $\quad$ Gradient elution for chromatography

Table 7: $\quad$ Optimized parameters for analysis of analytes by LC/MS/MS 
Table 8: $\quad$ Linearity, limit of detection (LOD), and lowest limit of quantitation (LLOQ) for the analytes

Table 9: $\quad$ Bias and precision data for the analytes tested

Table 10: $\quad$ Matrix effect, recovery, and process efficiency for analytes tested

Table 11: $\quad$ Freeze/thaw stability for three cycles over 72 hours for pooled fortified matrix

Table 12: $\quad$ Room temperature autosampler stability for processed samples over 72 hours for the pooled fortified matrix for analytes

Table 13: $\quad$ Freezer stability of processed samples over 72 hours for pooled fortified matrix 


\section{LIST OF ABBREVIATIONS}

AFM

$\mathrm{Ag} / \mathrm{AgCl}$

ASB

AdSV

AdS-SWV

CSWV

CV

CYP450 or CYP

CYP2D6

CYP3A4

DEA

dMRM

DPV

DUI

DUID

EDC

EDS

EIC

FA

FIA

FIBF

fSPCE

GC-MS

GCM-OPFP

HPLC-AD

IPA

ITO

LC-MS

$\mathrm{LC} / \mathrm{MS} / \mathrm{MS}$

LOD
Atomic Force Microscopy

Silver-Silver Chloride Reference Electrode

American Academy of Forensic Science Standards Board

Adsorptive Stripping Voltammetry

Adsorptive Stripping Square-Wave Voltammetry

Cyclic Square-Wave Voltammetry

Cyclic Voltammetry

Cytochrome P450

Cytochrome P450 Subfamily 2D6

Cytochrome P450 Subfamily 3A4

Drug Enforcement Administration

Dynamic Multiple Reaction Monitoring

Differential Pulse Voltammetry

Driving Under the Influence

Driving Under the Influence of Drugs

$N$-(3-dimethylaminopropyl)- $N$ '-ethylcarbodiimide

Energy Dispersive X-ray Spectrophotometry

Extracted Ion Chromatogram

Formic Acid

Flow Injection Analysis

para-Fluoroisobutyryl Fentanyl

Flexible Screen-Printed Electrode

Gas Chromatography-Mass Spectrometry

Glassy Carbon Microspheres N-octylpyridium tetrafluoroborate

High-Performance Liquid Chromatography Anodic Stripping

Isopropyl Alcohol

Indium Tin Oxide

Liquid Chromatography-Mass Spectrometry

Liquid Chromatography-Tandem Mass Spectrometry

Limit of Detection 
LLOQ

LSD

MRM

MWCNT

$\mathrm{NADH}$

$\mathrm{NADPH}$

NHS

NP

NPS

$\mathrm{OF} / \mathrm{P}$

PBS

RTIL

SAM

SEM

SPCE

SPE

SWGDRUG

SWV

TBAP

TIC

w.e.
Lowest Limit of Quantitation

Lysergic Acid Diethylamide

Multiple Reaction Monitoring

Multi-walled Carbon Nanotubes

Nicotinamide Adenine Dinucleotide

Nicotinamide Adenine Dinucleotide Phosphate

$N$-hydroxysuccinimide

Nanoparticles

Novel Psychoactive Substance

Oral Fluid-Plasma Partition Ratio

Phosphate Buffered Saline

Room Temperature Ionic Liquid

Self-Assembled Monolayer

Scanning Electron Microscope

Screen-Printed Carbon Electrode

Solid Phase Extraction

Screen-Printed Electrode

Scientific Working Group for the Analysis of Seized Drugs

Square-Wave Voltammetry

Tetrabutylammonium Perchlorate

Total Ion Chromatogram

Working Electrode 


\section{Part I: Introduction}

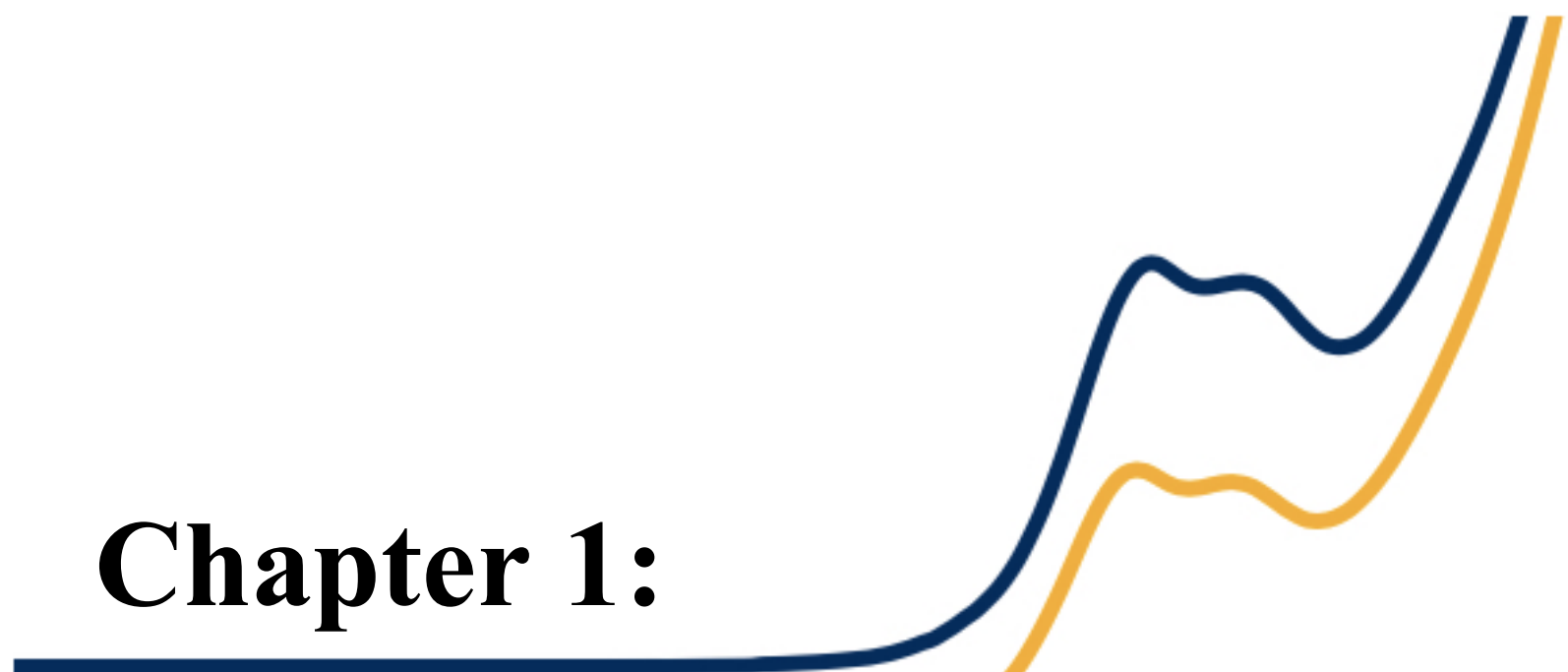

Purpose of Research and Objectives 


\subsection{Problem Statement and Purpose of the Study}

Opioids pose a significant threat to the health, order, and structure of society within the United States of America. Over the past several years, the increased use of opioids and novel psychoactive substances has resulted in what has been called the opioid epidemic, or the opioid crisis, and poses one of the most significant drug threats to the country. In response to the growing and staggering number of deaths, the United States Department of Health and Human Services declared a public health emergency. ${ }^{1}$ Although this emergency was first declared in 2017 , the public health emergency has been continuously renewed quarterly, the latest of which was in April 2019. ${ }^{2}$ Statistics regarding the nation's problem with opioids are overwhelming. It is estimated that over 130 people died every day from a drug overdose related to opioids in 2017 and 2018. Of the over 47,000 deaths, 15,000 were due to heroin while over 28,000 were attributed to synthetic opioids. On top of this problem, the misuse of prescription opioids has only exacerbated the problem. In 2016, over 11 million people misused prescription opioids contributing to their estimated involvement in opioid overdoses of $40 \%{ }^{1,3}$

This problem has ravaged the country, making it difficult to find an area that has not been affected by the opioid epidemic. However, West Virginia has suffered dramatically as the crisis has continued to unfold. In February 2018, as the struggle to curb the epidemic continued, West Virginia Governor, Jim Justice, requested the aid of the National Guard to combat the opioid problem. Justice stated that there is a "terrible drug epidemic," and said, "If we don't [stop the epidemic] it will cannibalize us." "4 In both 2016 and 2017, West Virginia had the highest per-capita drug overdose rate in the United States, growing from 52 to 57.8 deaths per 100,000 people. The difference between the second-highest state (Ohio) and West Virginia was greater than 10 deaths per $100,000 .^{5}$ The opioid problem has been prevalent in West Virginia for many years. In 2012, 96-143 prescription opioids were prescribed per 100 people and heroin use between 2014 and 2015 was higher than the national average. ${ }^{6}$ While the state, and country as a whole, struggle with heroin and prescription opioids, these more recognizable drugs have been overshadowed by the significant rise in novel psychoactive substances (NPSs).

NPSs are synthetic analogs of controlled substances that elicit a similar or heightened response compared to their controlled substance counterparts. These NPSs are prominent throughout a variety of drug classes including opioids, cannabinoids, and cathinones. ${ }^{7}$ NPSs are generally designed and synthesized by utilizing a core structure or another compound and making 
modifications to the core structure or compound. Among these NPSs, recent years have seen significant increases in fentanyl and fentanyl-related compounds, including many overdose deaths relating to these compounds. Due to the potency of fentanyl ( $\sim 100$ times more potent than morphine), fentanyl analogs (a category of NPSs) pose a significant threat to the country. ${ }^{8}$ In 2016 , 15 different fentanyl and fentanyl-like substances were seized by the DEA. Of these 15 substances, $9(60 \%)$ were reported for the first time. ${ }^{9}$ The appearance of new drug entities has not been an uncommon trend in recent years. In fact, due to the possible modifications of the core structure of fentanyl, it is estimated that there are approximately 2,000 potential fentanyl derivatives (Figure 1). ${ }^{8}$ These synthetic opioids (fentanyl analogs) have factored into the statistics mentioned above, resulting in a $107 \%$ increase in synthetic opioid-involved overdose deaths in West Virginia between 2015 and $2016 .^{10}$

The existence of a large number of isomeric forms poses many challenges for both the forensic science community and for the legal system as a result of the many modifications possible. First, due to the recent and ever-expanding discovery of new synthetic drugs, presumptive tests are either nonexistent, lack specificity, or are not safe as the drugs have to be handled with care. ${ }^{9}$ Scientific knowledge must be continuously updated regarding these compounds as more are discovered. The frequent incorporation of new entities into the illegal drug market often results in the lack of information concerning the testing and analysis of samples. Second, fentanyl analogs are generally found either as adulterants in low concentration and complex mixtures or in low purity. ${ }^{9}$ These compounds are commonly present in samples of heroin or cocaine to increase the effects of the drug. ${ }^{5}$ Lastly, there is a need for more research regarding the detection of these analogs for improved structure verification and identification. ${ }^{9}$ Current methods for achieving these identifications involve confirmatory analysis techniques that are complex, time-consuming, and expensive. Traditionally, chromatographic separation with mass spectrometry has been the analytical technique for confirmatory analysis in forensic laboratories because it provides excellent sensitivity and selectivity for differentiating multiple drugs simultaneously. However, there is a need for techniques that can minimize sample preparation, be performed outside of the laboratory setting, and can provide results quickly.

An analysis technique of this type would lend itself well toward a fast, qualitative analysis of drugs in the field with quasi-quantitative data, as well as, rapid analysis of samples and/or drivers for crime scene or driving under the influence of drugs (DUID) scenarios. Crime scene and 
DUID scenarios serve as useful models due to the need for immediate results that can provide information for officer and personnel safety, investigative leads, and cause for arrest. Furthermore, DUID cases could benefit from noninvasive collections of oral fluid at the scene, providing information about recent drug use and minimal time between collection and testing. The use of electrochemistry holds great promise for these types of applications due to the sensitivity, portability, low cost, and simplicity of instrumentation inherent in electrochemical methodologies, ${ }^{12}$ in addition to the use of disposable testing substrates preventing contamination. This type of methodology would prove useful in enhancing the forensic toxicology and seized drug workflow to reduce backlogs and cost, as well as, to speed up screening. The use of electrochemical methods for the analysis of drugs has been the topic of considerable interest to exploit the abovementioned qualities. The detection of drugs such as cannabis, ${ }^{13}$ lysergic acid diethylamide (LSD) ${ }^{14}$ cocaine,${ }^{15-17}$ synthetic cathinones, ${ }^{18}$ codeine, ${ }^{19}$ and heroin ${ }^{20}$ have been suggested. As such, electrochemical analysis offers a versatile platform that is currently not used to its full potential for detecting a wide array of substances. 


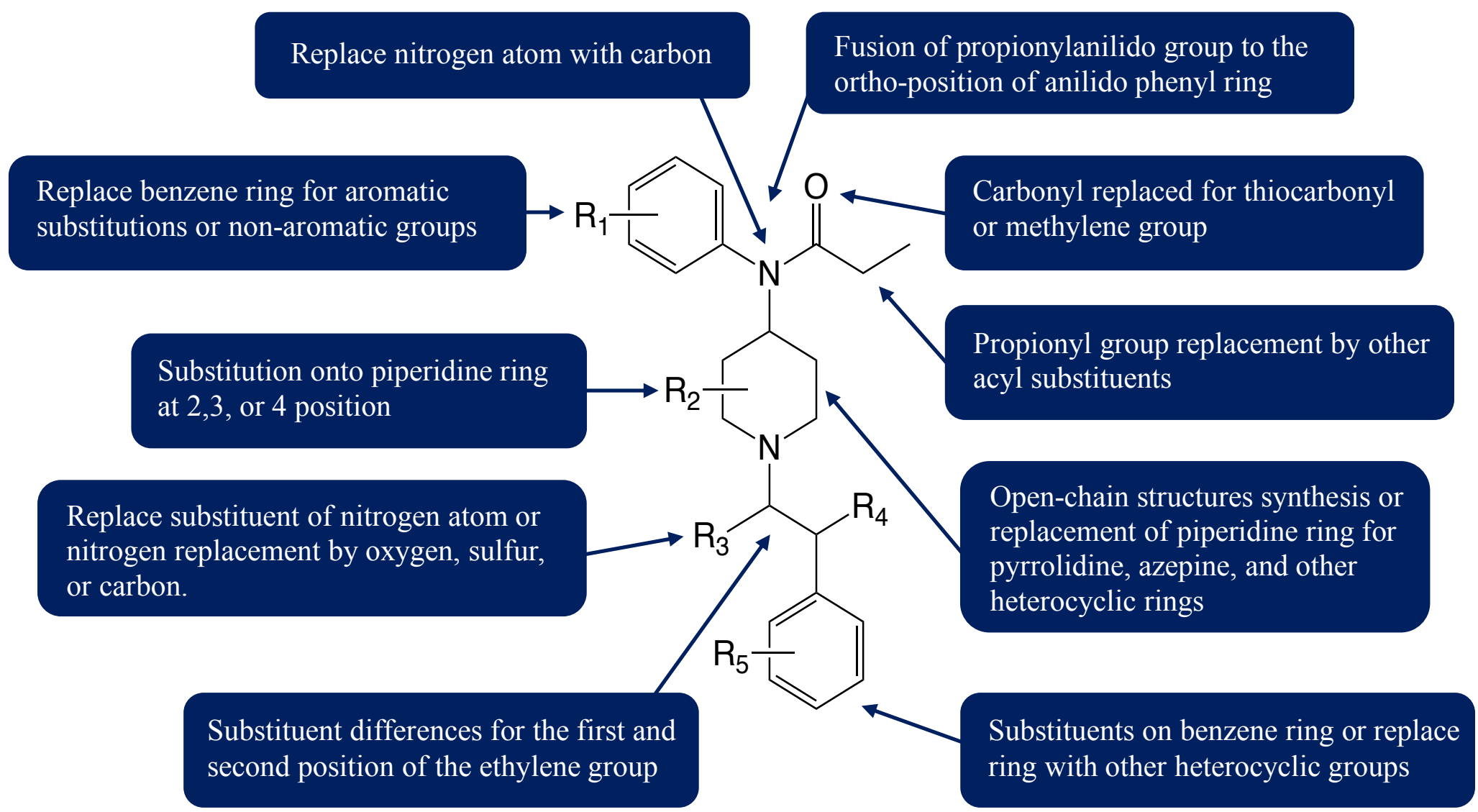

Figure 1: Potential modification sites of fentanyl. ${ }^{11}$ (Figure Adapted from Vardanyan et al.) 


\subsection{Goals and Objectives}

This study hypothesizes that the electrochemical oxidation/reduction process of opioids and novel psychoactive substances, including fentanyl and fentanyl analogs, is possible and will offer opportunities for rapid detection, identification, and field analysis. For this method to be relevant, accurate, qualitative information about the identification of a substance or sample must be acquired. The developed methodology should be capable of providing qualitative and quasiquantitative information about fentanyl alone and in the presence of other substances.

The development and testing of the electrochemical detection of fentanyl is presented in Part II of this thesis as a rapid and portable method. This method represents a simple, sensitive, and straightforward approach toward the analysis of the target analyte. Validation of the method, including figures of merit, is presented in chapter 3 of Part II along with the proposed electrooxidative mechanism for fentanyl under the methodology.

A confirmatory analysis method is proposed in Part III of this thesis utilizing liquid chromatography-tandem mass spectrometry for analysis of a suite of fentanyl and fentanyl analogs. The chosen matrix for this work was oral fluid to allow for applications in cases involving DUID. This matrix allows for non-invasive sampling and provides information about recent drug use. Chapters 2 and 3 of Part III outline the development, optimization, and validation of the confirmatory method in oral fluid according to the American Academy of Forensic Science Standards Board (ASB) following Standard 036.

The main objective of this study was to develop an electrochemical testing method for the identification of fentanyl, as well as, a confirmatory method for identifying fentanyl and its analogs in oral fluid using LC/MS/MS. This goal was achieved through the completion of the following sub-objectives:

1. Development of a rapid and straightforward electrochemical method that could be used in the field rather than in the laboratory.

2. Optimization of electro-analytical parameters to achieve optimal detection of fentanyl under seized drug case scenarios.

3. Adaptation of the analytical method to provide both qualitative and quasi-quantitative data for the identification of fentanyl. 
4. Validation of the analytical method, including the assessment of interfering compounds at various ratios.

5. Implementation of an extraction procedure in oral fluid for LC/MS/MS analysis.

6. Development and validation of a confirmatory analysis method with LC/MS/MS of oral fluid samples for an extended group of fentanyl analogs containing:
a. 2'-fluoro ortho-Fluorofentanyl
h. Furanyl fentanyl
b. 4-ANPP (Despropionyl fentanyl)
i. Methoxyacetyl fentanyl
c. Acetyl fentanyl
j. Norfentanyl
d. Acryl fentanyl
k. para-Fluroisobutyryl fentanyl
e. Carfentanil
1. U-47700
f. Despropionyl para-Fluorofentanyl
m. Valeryl fentanyl
g. Fentanyl

Without interference from:
a. 6-monoacetylmorphine
g. Hydrocodone
b. Amphetamine
h. Methadone
c. Benzoylecgonine
i. Methamphetamine
d. Buprenorphine
j. Morphine
e. Codeine
k. Norbuprenorphine
f. Ethylmorphine
1. Oxycodone

Lastly, Part IV outlines and summarizes the principal conclusions of the overall thesis and reiterates findings of both the electrochemical detection of fentanyl and the LC/MS/MS method for the suite of fentanyl and fentanyl-like substances, while Part V of this document demonstrates various other electrochemical approaches toward the detection of codeine, fentanyl, and PB-22 that were explored in the pursuit of this research study. 


\section{Part II: \\ Electrochemical \\ Analysis of Fentanyl}

Chapter 1:

Relevant Literature Review 


\subsection{Opioids}

For hundreds of years, people have been using natural opioids for their medicinal and psychological properties. Natural opioids, such as morphine, are derived from the poppy Papaver somniferum and have traditionally been referred to as narcotics. ${ }^{21}$ Although this term is still sometimes used, the terms opiates and opioids have become more commonplace, with "opioids" commonly being used to refer to all drugs within this class. This class of drugs contains the natural opioids, as well as, semi-synthetic opioids such as oxycodone, heroin, and hydrocodone which are derived from the natural opiates, and synthetic opioids manufactured entirely in the laboratory to mimic the effects of opioids. ${ }^{21}$

Although currently causing many problems in society, opioids are an especially useful tool in the medical field for pain relief ${ }^{22}$ and sedation. ${ }^{21}$ These drugs owe their effects to their ability to interact with the opioid receptors in the body and the brain to decrease the body's response to pain. However, these drugs also produce a euphoric effect ${ }^{23}$ in users and high physical and psychological dependence, which is responsible for their current use and abuse in society.

\subsection{Opioid Metabolism}

The majority of substances that enter the human body must eventually be excreted from the system. Xenobiotics, including opioids, must undergo the process of metabolism within the body. The metabolic process is the pathway for the biotransformation of drugs within the body, resulting in alterations from their original state. ${ }^{22}$ The metabolic process is of great importance and interest to many parties, including researchers and pharmaceutical companies who can target specific chemical conversions within the body for their products.

Although the body performs metabolic processes with the intent of removing the xenobiotic, the results of metabolism can be unwanted. The metabolism of some opioids can result in the production of active metabolites that possess properties sometimes to a higher degree than the pre-metabolized drug. ${ }^{22}$ The opioid metabolic process, and for most drugs, begins with firstpass metabolism, consisting of phase I and phase II metabolism. First-pass metabolism takes place in the liver, generally before the circulation of a drug throughout the body. ${ }^{22}$ Phase I results in the chemical modification of the opioid that includes $\mathrm{N}-, \mathrm{O}-, \mathrm{S}-$ dealkylation; aromatic, aliphatic, or $\mathrm{N}$ - hydroxylation; $\mathrm{N}$ - oxidation; sulfoxidation; deamination; and dehalogenation. ${ }^{22}$ These processes occur through a general class of enzymes called cytochrome P450s. Opioids are 
primarily metabolized by two cytochrome isozymes, CYP3A4 and CYP2D6. ${ }^{22}$ CYP3A4 has been demonstrated to be the primary cytochrome P450 enzyme responsible for the metabolism of fentanyl through $\mathrm{N}$ - dealkylation to norfentanyl. ${ }^{24,25}$ Phase II metabolism consists of a process by which drugs are made more hydrophilic, referred to as glucuronidation, which serves to allow the drugs to be easily excreted by the body. ${ }^{22}$

\subsection{Fentanyl and Fentanyl Analogs}

Fentanyl, a basic compound, is considered a synthetic opioid and has been used in medical practice for surgical anesthesia since $1963^{26}$ and for the treatment of pain, ${ }^{11}$ especially for individuals with advanced cancer. ${ }^{27}$ Fentanyl can produce symptoms ranging from respiratory depression and seizures to $\operatorname{comas}^{26}$ to causing death at amounts as low as $2 \mathrm{mg}^{28}$ Fentanyl is commonly administered intravenously, subcutaneously, or through transdermal patches. ${ }^{27}$ This drug poses a real risk to society due to its potency which is approximately 100 times that of morphine. ${ }^{8}$ The half-life of fentanyl is approximately 3-4 hours with $85 \%$ being excreted within 4 days through the urine as fentanyl or its metabolites of norfentanyl, hydroxynorfentanyl, hydroxyfentanyl, ${ }^{26}$ and despropionylfentanyl (4-ANPP). ${ }^{29}$

Fentanyl was first synthesized by Paul Janssen and has since been extensively studied with various synthetic approaches and changes to the structure to produce derivatives of fentanyl. ${ }^{11}$ The main structural components of fentanyl include an amide group, aniline ring, piperidine ring, and an N-alkyl chain. (Figure 2).

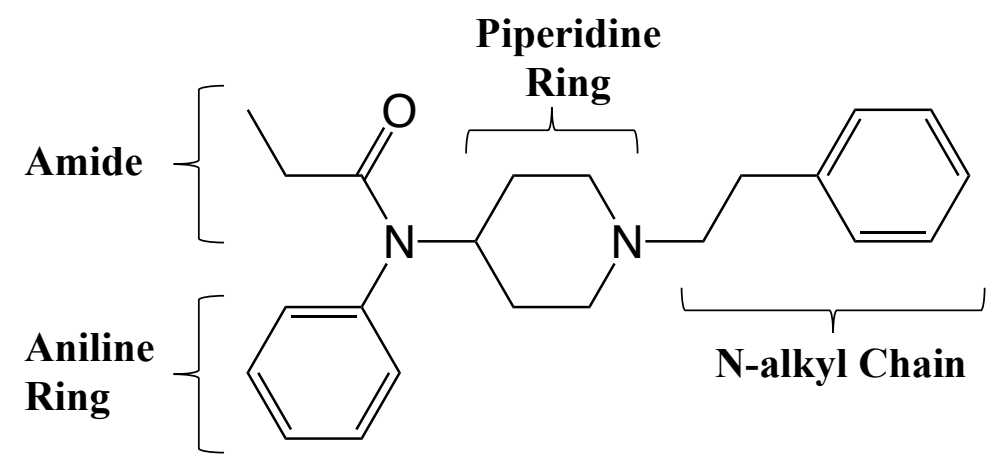

Figure 2: Fentanyl structure demonstrating scaffold regions as locations for modifications. ${ }^{29}$ (adapted from Cayman Chemical) 
As seen in Figure 2 above, there are many areas throughout the fentanyl structure allowing for modification and the development of a multitude of fentanyl analogs. Fentanyl acts through an affinity for the $\mu$-opioid receptors. ${ }^{11}$ Analog compounds are those compounds that illicit a similar or heightened response when compared to their parent molecule. One such fentanyl analog, carfentanil, has demonstrated heightened potency and is considered to be approximately 10,000 times more potent than morphine. ${ }^{8,11}$ Since approximately 2009, the number of newly reported novel psychoactive substances has increased drastically from previous years. ${ }^{8}$ Part of this increase can be attributed to the rise of these fentanyl analogs. As mentioned previously, due to the many locations within the fentanyl structure, it has been hypothesized that there are approximately 2,000 possible fentanyl analogs. ${ }^{8}$

Several groups have published data regarding the electrochemistry of fentanyl. These studies are summarized in brief within the table below (Table 1).

Table 1: Literature review of fentanyl detection via electrochemical methods

\begin{tabular}{ccccccc}
\hline Electrode & Technique & $\begin{array}{c}\text { LOD } \\
(\mu \mathrm{g} / \mathrm{mL})\end{array}$ & $\begin{array}{c}\text { Linear Range } \\
(\mu \mathrm{g} / \mathrm{mL})\end{array}$ & $\mathrm{R}^{2}$ & RSD (\%) & Reference \\
\hline GCM-OPFP & $\begin{array}{c}\text { CV/ Electrochemi- } \\
\text { luminescence }\end{array}$ & 0.003 & 0.003 to 33.6 & 0.9994 & $\begin{array}{c}1.9 \text { at } 1.7 \\
\mu \mathrm{g} / \mathrm{mL}\end{array}$ & 30 \\
Graphite SPE & HPLC-AD & 0.77 & 10 to 120 & 0.999 & 0.53 & 31 \\
Hg & ASV & 0.017 & 0.336 to 0.034 & 0.9992 & 3.6 & 32 \\
SPCE-RTIL & CSWV & 1.7 & 1.7 to 32 & 0.997 & -- & 33 \\
fSPCE-MWCNT/ & SWV & 3.4 & 3.4 to 33.6 & 0.992 & 3.2 & 34 \\
RTIL & AdS-SWV & 0.075 & 0.075 to 0.64 & 0.9948 & 12 & This work \\
SPCE & & & & & & \\
\end{tabular}

\subsection{Electrochemistry}

Electrochemistry refers to the study of the interactions between electricity and chemical changes. Many areas of chemistry utilize electrochemical concepts including synthesis, kinetics, nerve impulses, electron transport, and thermodynamics, among others. ${ }^{36}$ A specific type of chemical reaction, termed an oxidation-reduction (Redox) reaction, describes a chemical change 
resulting from the movement of electrons within a system. Redox reactions are characterized by the gain of electrons by one species (reduction) and the loss of electrons by another species (oxidation). Equation 1 demonstrates a simple example of a redox reaction:

$$
O+e^{-} \rightleftharpoons R
$$

where $O$ represents the oxidized species, $e^{-}$represents the number of electrons involved in the reaction, and $R$ represents the reduced species.

Electro-analytical measurements generally involve the measurement of current resulting from a chemical change, referred to as Faraday's Law. The use of Faraday's Law, the application of a Faradaic process, provides a relationship by which the concentration of the analyte in solution can be determined based on the amount of current flowing:

$$
Q=n F N
$$

where $\mathrm{Q}$ is the charge, $\mathrm{n}$ is the number of electrons in the reaction, $\mathrm{F}$ is Faraday's constant $(96,485$ $\mathrm{C} / \mathrm{mol}$ ), and $\mathrm{N}$ is the number of moles electrolyzed in the reaction. Through differentiating Equation 2, the relationship between current and analyte can be seen as:

$$
i=n F \frac{d N}{d t}
$$

where $\mathrm{i}$ is the current, and $\mathrm{dN} / \mathrm{dt}$ is the change in moles over the change in time. ${ }^{36}$

Electrochemistry is a mature analytical technique that has proven to be a versatile platform for analysis. Owing to this versatility is the variety of techniques and methods available, whose parameters of potential $(\mathrm{E})$, current $(\mathrm{I})$, charge $(\mathrm{Q})$, and time $(\mathrm{t})$ can be measured or controlled to provide the required information about the analyte in solution. Aside from the variety of methods, electrochemistry offers high sensitivity, accuracy, precision, ${ }^{37}$ and selectivity. For example, low detection limits are often possible with picomole analysis in some cases. ${ }^{36}$

However, to utilize electrochemistry for analytical purposes, the analyte of interest must be electroactive. Electroactivity can be thought of as the ability of a species to alter its oxidation state as a result of potential being applied. This redox process can be accomplished either through the innate properties of the analyte, employing a method to generate an electroactive species based on the analyte, or sensing the species indirectly with another electroactive mechanism. This idea 
results in one way to gain selectivity in electrochemical measurements. Standard reduction potentials describe the characteristic potential at which oxidation-reduction of specific species occurs.

\subsubsection{Electrodes}

Typically, electroanalytical techniques employ methods that utilize a potentiostat and three electrodes: the working electrode, the auxiliary/counter electrode, and the reference electrode. The potentiostat serves to control the potentials applied to the electrodes and also measures the resulting current within the chemical system, accomplished through the use of the three electrodes mentioned previously.

The working electrode serves as the location where the redox reaction of interest will take place. Working electrodes can be made of many different materials such as carbon, indium tin oxide (ITO), mercury, ${ }^{12}$ gold, platinum, etc. The choice of working electrode construction is tailored to the application of the investigator or the chosen technique. Working electrodes generally are inert materials with good conductivity and wide potential windows. ${ }^{36}$ The auxiliary electrode serves as the location of the complementary redox process to the working electrode. ${ }^{12}$ The auxiliary electrode is typically a non-polarizable metal such as platinum or palladium that allows current to flow freely across the electrode-electrolyte interface. Finally, the reference electrode serves to provide a constant potential from which the applied potential to the chemical system can be monitored and measured. ${ }^{12}$ These electrodes require a stable, highly reproducible and reversible half-cell potential and reaction. ${ }^{36}$ The most common form of reference electrode found today consists of silver-silver chloride $(\mathrm{Ag} / \mathrm{AgCl})$. Variations of these three types of electrodes allow for versatility in analysis procedures, sensitivity, and selectivity.

Traditionally, the field of electrochemistry has employed what is termed the conventional electrochemical cell (Figure 3). This type of system generally employs the three electrodes mentioned above, which are relatively large and cumbersome. While traditional electrodes can often provide very sensitive measurements, they usually require extensive cleaning and treatment between uses. Furthermore, the typical electrochemical cell requires large sample volumes for the electrodes to be immersed in the supporting electrolyte. Some limitations presented by the conventional electrochemical set-up have been addressed by the development of screen-printed electrodes (SPE). These electrodes encompass the three conventionally electrodes within a single 
platform that has a small footprint (Figure 3). Although SPEs can be manufactured using a variety of materials, a common form is the screen-printed carbon electrode (SPCE). SPCEs have many advantages over the traditional methods due to their small size including low cost, robustness, and disposability. ${ }^{38}$ Small volumes can be analyzed, leading to their use in analytical methods where large samples cannot be obtained or would be inappropriate/impractical. SPCEs also have good reproducibility between electrodes, allowing them to be disposable due to inexpensive manufacturing. Finally, the carbon substrate for the working electrode allows for many modifications and changes to be incorporated during or after the manufacturing process, supporting a wide range of applications. The small size of these electrodes makes them excellent candidates for point-of-care devices and sensors.
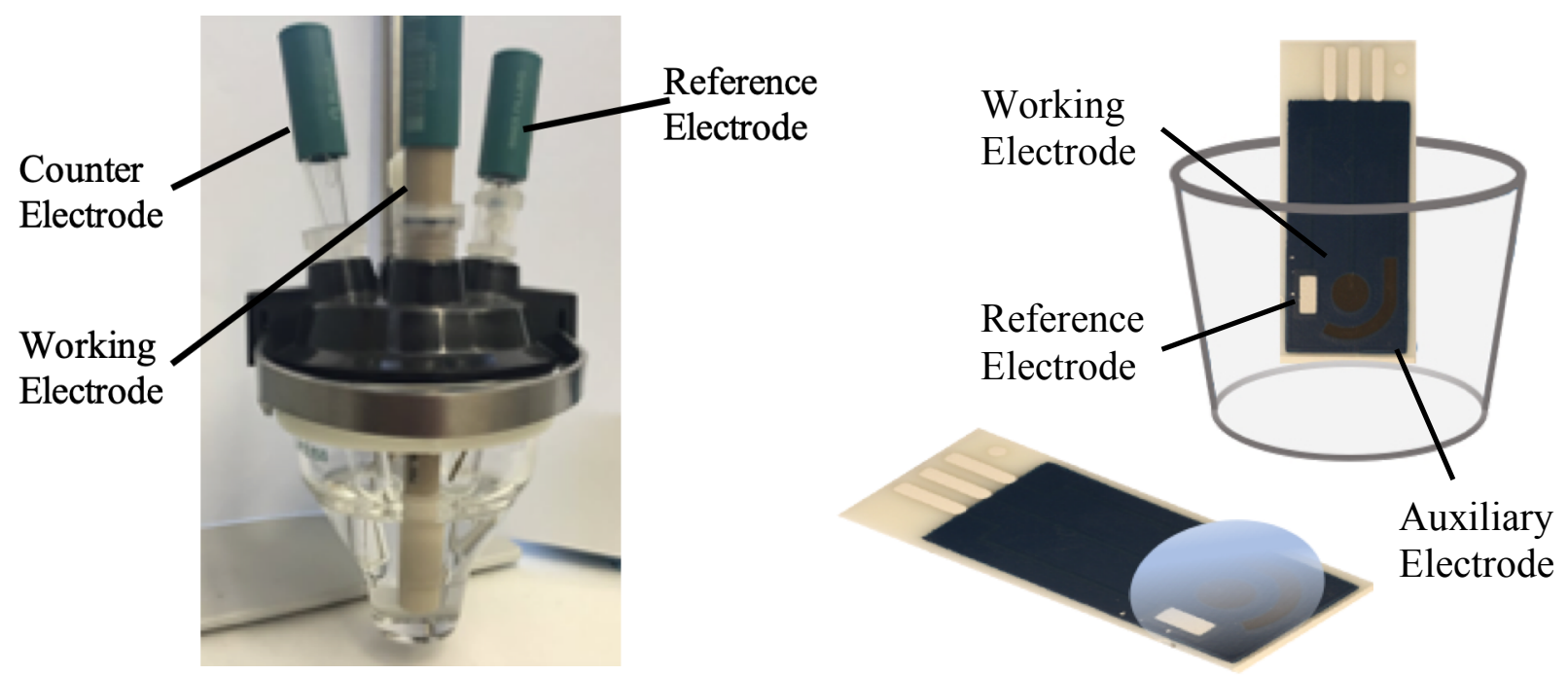

Figure 3: Cell set-up utilizing conventional electrodes with an approximate height of $15 \mathrm{~cm}$ (left) and screen-printed carbon electrodes used in this work with an approximate size of $3.5 \mathrm{~cm} \times 1.5 \mathrm{~cm}$ demonstrating use in cell or with a drop (right).

\subsubsection{Voltammetric Techniques}

Voltammetric techniques are of great importance and extensive use since they utilize a changing potential at a rate relative to the reference electrode and measure the resulting current between the working and reference electrodes termed a dynamic electroanalytical technique. ${ }^{36}$ Additionally, these methods can provide both qualitative and quantitative information about the species in solution. The resulting plot, termed a voltammogram, plots the current against the potential. Voltammetry is considered an active technique and has many parameters that can be varied for different purposes including waveform, stirring, time, and electrode parameters. ${ }^{36}$ 
Cyclic voltammetry (CV) is a commonly encountered voltammetric technique. CV utilizes a changing potential that is swept either in the positive or negative direction and then reversed and swept back in the other direction. Typically, this is a stationary technique where the solution is non-stirred (quiescent) and movement in solution is defined by diffusion. ${ }^{36}$ In this way, both reduction and oxidation processes can be observed that can provide the identification and characterization of an analyte. Due to the ability to quickly analyze a wide potential range, $\mathrm{CV}$ is often the first technique used during the investigation of an analyte. ${ }^{36}$ This approach can elucidate the electrochemical behavior of the analyte. CV operates through the use of a linearly changing potential. Figure 4 demonstrates an example of a cyclic voltammogram and the theoretical waveform used to generate the plot.
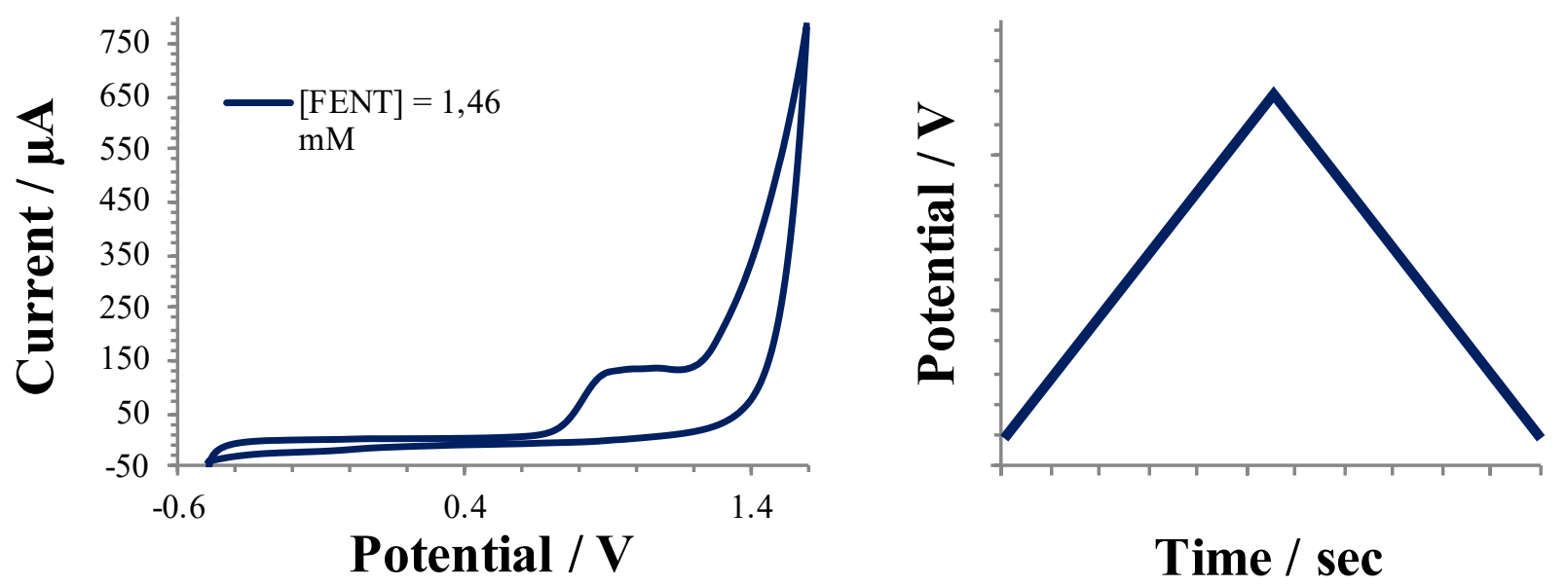

Figure 4: Cyclic voltammogram for fentanyl at $1.46 \mathrm{mM}$ (left) and waveform demonstrating the cyclic nature of the potential applied (right).

Two different pulse voltammetry techniques are commonly used in the analysis of species with electrochemistry: differential pulse voltammetry and square-wave voltammetry. These techniques rely on the pulsing of potentials to gain information about the analyte in solution. In summary, the potential is quickly increased to a value, held for a short time, and then dropped again. This process repeats with increasing potential, resulting in the probing across a potential range. ${ }^{36}$ These techniques are commonly used when low detection limits are desirable or required. Measurement of the current within the system occurs toward the end of each pulse, allowing the charging current to be minimized as it will decrease faster than the faradaic current. ${ }^{36}$ 
Differential pulse voltammetry (DPV) utilizes the pulse technique in a way that allows the potential to be scanned. Rather than dropping the potential back down to a baseline value, the potential drops less than the original pulse. ${ }^{36}$ In this way, the potentials of the pulse are increased continuously over the desired potential window. However, of interest to this study is square-wave voltammetry.

Square-wave voltammetry (SWV) is another pulse technique. As with other pulse techniques, SWV attempts to minimize the effect of charging current. This technique implements a potential waveform that can be thought of as constant incremental pulses superimposed on a staircase (Figure 5). ${ }^{36}$ This is to say that the pulse follows a step-wise increase in potential for both the forward pulse and reverse pulse. The effect of charging current is minimized through the sampling of current response near the end of the forward pulse and then again near the end of the reverse pulse. The voltammogram is then constructed by plotting the net current of the forward and reverse pulse, resulting in a peak. ${ }^{36}$ Utilizing SWV allows a single scan to achieve both oxidation and reduction (in the case of a reversible process) when the redox potential of the analyte falls between the potential step, allowing for the use of the net current generated. SWV is a widely used technique due to its ability for rapid scan rates and low limits of detection, often in the $10^{-7}$ $\mathrm{M}$ to $10^{-8} \mathrm{M}$ range.

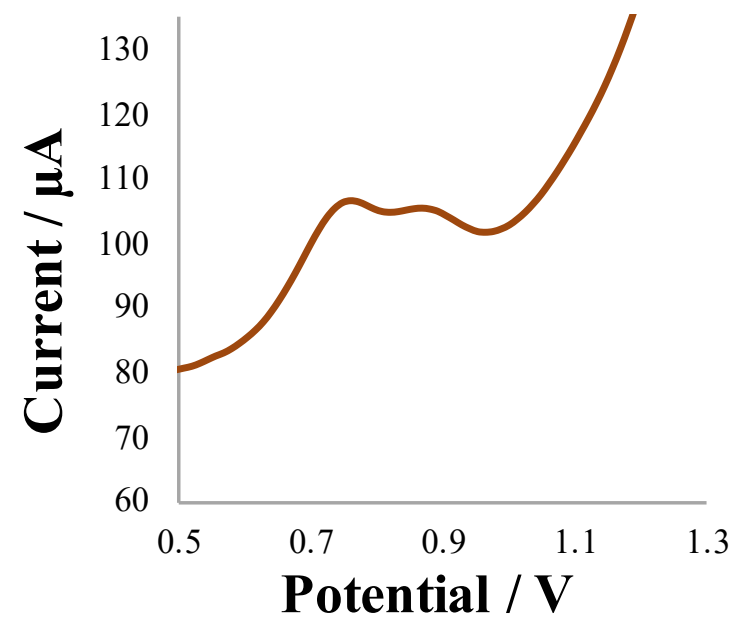

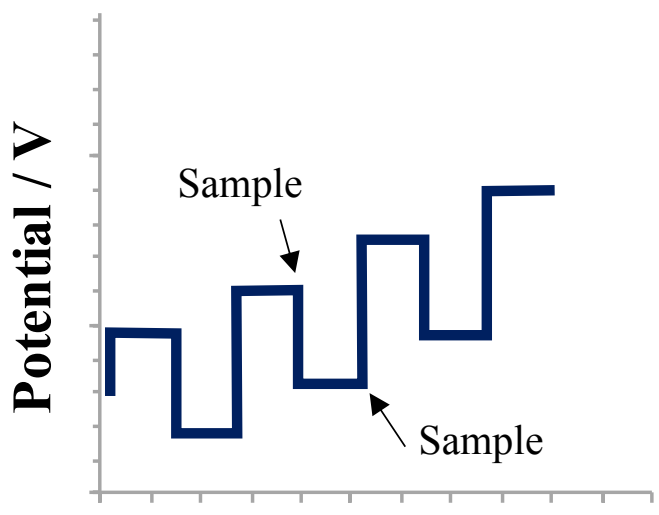

Time / sec

Figure 5: Adsorptive stripping square-wave voltammogram for fentanyl at $3.62 \mu \mathrm{g} / \mathrm{mL}$ (left) and waveform demonstrating a pulsed signal superimposed on a staircase giving the forward and reverse waves (right).

Lastly, electrochemistry and voltammetry rely on the ability of the current to move throughout the system. Therefore, the concept of using a supporting electrolyte is essential. The 
selection of the supporting electrolyte can be critical for the analysis being performed for several reasons. First, the electrolyte lowers the resistivity within the system. Low resistivity allows current to flow effortlessly throughout the system. ${ }^{36}$ Second, the electrolyte can minimize the effect of migration within the system that could affect mass transport. In order to achieve this, it is generally recommended that the supporting electrolyte be at a concentration approximately 100 times the concentration of the analyte. ${ }^{36}$ Lastly, the electrolyte can have an effect on the electrochemical processes and can be used to influence redox potentials in order to better separate analyte peaks. ${ }^{36}$

\subsubsection{Amperometric Techniques}

Amperometric techniques make use of a single potential and are thus considered a potential step technique, where the potential is "stepped" to a particular value and then held for a certain amount of time. ${ }^{36}$ Therefore, the waveform for the potential applied during chronoamperometry is a horizontal line. The current is then measured against time while the potential is held constant. While chronoamperometry generally makes use of a quiescent solution, amperometric detection can also be achieved for a stirred system. This technique can be useful for a standard addition approach for quantitative analysis due to the ability to see changing current at certain times. Amperometric techniques are commonly encountered in methods utilizing anodic or cathodic stripping voltammetry. Voltammetric stripping techniques utilize a constant applied potential for a set period to deposit the analyte onto the working electrode surface. A subsequent voltammetric technique then sweeps the potential in order to strip, or remove, the analyte from the working electrode through the redox process. This deposition of analyte material serves as a preconcentration step, allowing for the increased sensitivity that voltammetric stripping techniques enjoy. 


\subsection{Forensic Electrochemistry}

A pseudo-branch of electrochemistry, forensic electrochemistry describes the use of electrochemical methods and techniques to monitor response as a result of oxidation or reduction of an analyte relating to evidence found at the scene of a crime or used during a criminal act with the principle intention of providing qualitative or quantitative information. ${ }^{12}$ Electrochemistry proves useful in forensic applications due to its sensitivity, selectivity, low cost, and portability. Electrochemical techniques have already been reported for a wide range of forensic applications including poisons, gunshot residue, fingerprints, DNA, and drugs. ${ }^{12}$ Of interest to this thesis is the application of electrochemical techniques for the detection of drugs of abuse.

The utilization of electrochemical techniques for the detection of drugs of abuse has been shown in the literature. For examples, Jiang et al. reported a sensitive detection technique for cocaine using an "aptamer-based sandwich" along with a graphene/AuNP modified screen-printed carbon electrode (SPCE). The addition of multiple molecules including NADH, streptavidinconjugated alkaline phosphatase, and $p$-aminophenylphosphate, among others allowed for an assay format and redox-recycling that provided an estimated limit of detection of $1 \mathrm{nM} .{ }^{15}$ Oliveira et al. reported a fast, non-destructive voltammetric method for detecting cocaine. This approach utilized methoxy-substituted N,N'-ethylene-bis(salcylideneiminato)uranyl(VI) complexes to modify a carbon paste electrode along with cyclic voltammetry. The authors reported a working range of $1.0 \times 10^{-7} \mathrm{M}$ to $1.3 \times 10^{-6} \mathrm{M} .{ }^{16}$ Finally, Asturias-Arribas et al. reported cytochrome P450 2B4 based electrochemical biosensor for the detection of cocaine with an SPCE. Covalent attachment of the enzyme allowed the authors to achieve direct electron transfer to the enzyme and provided a detection limit of $0.2 \mathrm{mM} .^{17}$

Lowe et al. demonstrated that tetrahydrocannabinol could be detected utilizing an edge plane pyrolytic graphite electrode. This indirect detection of THC was performed at low potential compared to the typical $1.2 \mathrm{~V}$ and resulted in a limit of detection of $25 \mu \mathrm{M} .{ }^{13}$ Balbino et al. demonstrated the usefulness of electrochemical methods to decrease false positives. By utilizing a glassy carbon disk electrode and a pre-concentration step, the authors were able to detect $\triangle 9$-THC in the presence of the FBB color test reagent using cyclic voltammetry and obtained a limit of detection and quantitation of $1 \mathrm{ng} / \mathrm{mL}$ and $3.5 \mathrm{ng} / \mathrm{mL}$, respectively. ${ }^{39}$ 
Merli et al. developed a method utilizing stripping voltammetry for the determination of lysergic acid diethylamide (LSD). Adsorptive stripping voltammetry with a glassy carbon electrode allowed the authors to determine the presence of LSD quantitatively. A linear range of 1-90 ng/L with a limit of detection of $1.4 \mathrm{ng} / \mathrm{L}$ and limit of quantitation of $4.3 \mathrm{ng} / \mathrm{L}$ were obtained and were comparable to LC-MS. The authors also investigated potential interfering species commonly administered with LSD and saw no interferences at 50 times the LSD concentration. ${ }^{14}$

Smith et al. investigated the electrochemical behavior of synthetic cathinones using boron-doped diamond, glassy carbon, and screen-printed graphite electrodes. The authors utilized $( \pm)$-methcathinone, $( \pm)-4$ '-methylmethcathinone, $( \pm)$-mephedrone, and $( \pm)-4$ '-methyl$N$-ethylcathinone. The authors demonstrated the electroactivity of cathinone species; however, problems with the selectivity of mixtures were reported. ${ }^{18}$

Finally, of interest to this study, several articles have been published concerning the electrochemical behavior of opioids. Garrido et al. investigated the oxidative behavior of heroin and its metabolites using differential pulse voltammetry and characterized the electrochemical mechanisms, proposing a new mechanism for the oxidative production of a secondary amine and an aldehyde from heroin. ${ }^{20}$ Asturias-Arribas et al. demonstrated the ability to utilize an enzyme-modified screen-printed carbon electrode for the detection of codeine. The authors used the CYP2D6 isozyme as the biological recognition molecule and covalently bound the enzyme to a modified electrode surface for direct electron transfer. A limit of detection of $4.9 \mu \mathrm{M}$ and linear range of 4.9-45.4 $\mu \mathrm{M}$ was achieved. The authors also demonstrated the use of the technique in the analysis of spiked urine samples, as well as, the reproducibility and repeatability of the methodology. ${ }^{19}$

\subsection{Electrochemical Biosensors}

Biosensors consist of an analyte-specific biological molecule. The biosensor could be an enzyme, antibody, receptor, etc. They also contain a transducer which allows some change to occur that can be monitored by a detector. ${ }^{40}$ The study of biosensors for forensic science applications is relatively new. Immunoassays have been the preferred choice historically for illicit drug biosensors and have had much success. They have been integrated into chip forms with antibody-arrays for 
testing multiple drugs, enhancing selectivity and specificity, as well as, eliminating false-positives. Several commercial tests utilizing biosensors have seen cut-off levels for opiates ranging from 40 $\mathrm{ng} / \mathrm{mL}$ to $10 \mathrm{ng} / \mathrm{mL} .{ }^{40}$ However, other forms of biosensors have been explored and are currently being investigated in more detail.

Electrochemical biosensors based around enzyme coupled reactions utilize the enzyme to either produce an electroactive species or deplete an electroactive species in such a way that an electrical signal can be produced. ${ }^{41}$ In addition to sensitivity, simplicity, low cost, and fast response time, the use of enzymes imparts another layer of selectivity toward the targeted analyte but also determines the lifespan of the sensor. ${ }^{41-44}$

The utilization of CYP biosensors has taken many forms historically. The major problem surrounding these studies is how to make the CYP biosensor more effective. Some have used $\mathrm{NADPH}$ as the electron mediator. However, to eliminate the need for a mediator, immobilization of the CYP enzyme on the electrode surface has been recently suggested. The evolution of these electrodes has gone through three generations, according to Putzback. ${ }^{41}$ First and secondgeneration electrochemical biosensors suffered from poor electron transfer, leaching of mediators, interference problems, and the need for oxygen. Third generation biosensors have attempted to eliminate these problems, mainly by immobilizing the mediator and/or enzyme to the surface of the electrode. These sensors generally boast increased electron transfer efficiency, faster response times, and higher sensitivities. ${ }^{41}$ Further, electrode modifications can help achieve these characteristics and amplify them. ${ }^{41}$

Various groups have explored different strategies including adsorption on bare electrodes, layer-by-layer adsorption, adsorption to thin films, the use of screen-printed electrodes, encapsulation in polymers or gels, covalent attachment to self-assembled monolayers, and nanostructured materials. ${ }^{45}$ There have been many examples of the use of CYP biosensors and enzymes utilizing these techniques. However, immobilization of the enzyme may cause random enzyme orientation resulting in a less accessible active site with less favorable electron transfer ${ }^{41}$ or conformational changes to inactive forms. ${ }^{45}$

Nanoparticle modifications have been a popular choice due to their ability to increase the number of biological molecules on the surface of the electrode. ${ }^{41}$ The choice to use nanomaterials is made in part because of their size-tunable properties, high surface-to-volume ratio, shapedependent properties, reduced energy consumptions, etc. ${ }^{46}$ Nanoparticles have been shown to 
improve performance for biosensors due to their comparable size to biomolecules and analytes, and are useful in their ability to provide proper orientation, proper interfacing, to serve as electrodes, and to provide better electron transfer. ${ }^{46}$ These benefits demonstrate improved sensitivity, limit of detection, and selectivity of biosensors. ${ }^{46}$ Many groups have demonstrated the use of nanomaterials to stabilize the active forms of biomolecules and to immobilize these molecules to the electrode surface. ${ }^{46}$

A conventional method, due to its simplicity, is covalent attachment utilizing selfassembled monolayers (SAMs). The use of SAMs imparts functional groups $\left(-\mathrm{CH},-\mathrm{NH}_{2}\right.$, or - $\mathrm{SH}$ ) to the surface of the electrode for the covalent attachment of the enzymes. SAMs of long and short size have also been utilized to increase electron transfer rates. ${ }^{41}$ Yang et al. demonstrated one such use through an 11-mercaptoundecanoic acid and octanethiol SAM on a gold electrode for the sensing of warfarin. The authors eliminated the need for cofactors and mediators and demonstrated the direct electron transfer between the heme group of the covalently bonded CYP2C9 and the gold electrode. ${ }^{47}$ Another SAM technique utilizes amine coupling. This technique uses a monolayer with amine groups and the addition of EDC (1-Ethyl-3-[3-dimethylaminopropyl] carbodiimide hydrochloride) and NHS (N-hydroxysuccinimide) to covalently bond the enzyme to the monolayer. ${ }^{45}$

\subsection{Cytochrome P450}

The term Cytochrome P450 (CYP) describes a family of heme-containing enzymes that share a similar structure (Figure 6). Many isozymes, various forms of the CYP enzyme, have been discovered and studied. ${ }^{45}$ These enzymes were previously referred to as mixed-function oxidase enzymes. This name was based on the fact that they reside on the membranes of the endoplasmic reticulum within the liver and are the terminal oxidases of the mixed-function oxidase system. The current name represents the wavelength at which the enzymes absorb light (450 nm) with 'p' standing for the word pigment. ${ }^{48}$ CYPs play a critical role in the metabolism of many xenobiotics (foreign substances in the body, such as drugs). Metabolic processes mentioned earlier such as hydroxylation, epoxidation, and $\mathrm{N}-, \mathrm{S}-$, and $\mathrm{O}-$ demethylation are a result of CYP metabolism ${ }^{45}$ and an attempt to increase the water solubility of the drug. ${ }^{48}$ The pharmaceutical industry has invested much effort into the understanding of the inhibition and substrates of these 
enzymes. ${ }^{45}$ In mammals, 18 different CYP families have been discovered, and 43 subfamilies in humans with 57 individual enzymes have been found. These various isozymes are denoted by a combination of a number-letter-number system, where the first number and letter designate the amino acid sequence and the final number is the enzyme discovery number (i.e., CYP3A4). ${ }^{48}$ Of the various CYP enzymes detected, 90\% of drug oxidation is due to 6 enzymes, including 2D6 and $3 \mathrm{~A} 4$.

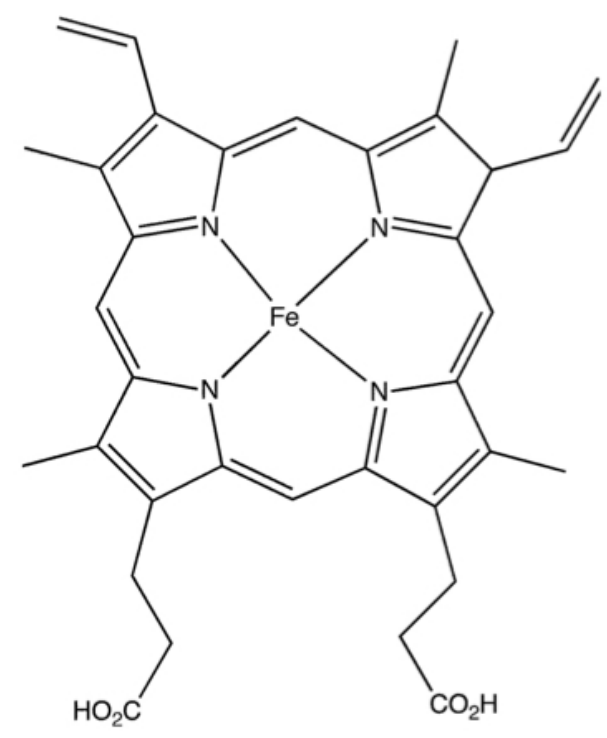

Figure 6: Heme (iron protoporphyrin IX) group. ${ }^{49}$

These CYP enzymes follow a typical catalytic cycle (Figure 7) due to the presence of the heme group, resulting in a two-electron reduction of the enzyme. Upon entering the active site of the enzyme, the substrate displaces the bound water ${ }^{50}$ inducing a high-spin $\mathrm{Fe}^{3+}$ from the low-spin resting state (although the spin shift is not always needed in all systems). ${ }^{51}$ This results in an out of plane iron by $0.3 \AA$ and a pentacoordinate heme iron (a). ${ }^{51}$ A more positive reduction potential results, allowing for electron transfer, and the ferric enzyme is reduced to a ferrous state (b). ${ }^{50}$ Oxygen then binds resulting in the ferric state and a low-spin hexacoordinated iron (c). ${ }^{51}$ Further reduction of the complex results in a peroxo-intermediate (d) followed by the protonation to a hydroperoxo-intermediate (e). ${ }^{50} \mathrm{~A}$ second protonation with heterolysis results in an oxoferryl species (highly reactive) (f). ${ }^{51}$ A substrate radical is then formed by the loss of substrate hydrogen 
to the oxoferryl species (g), which hydroxylates the substrate through radical rebound, resulting in its release from the enzyme and the low spin state of the enzyme (h). ${ }^{51}$

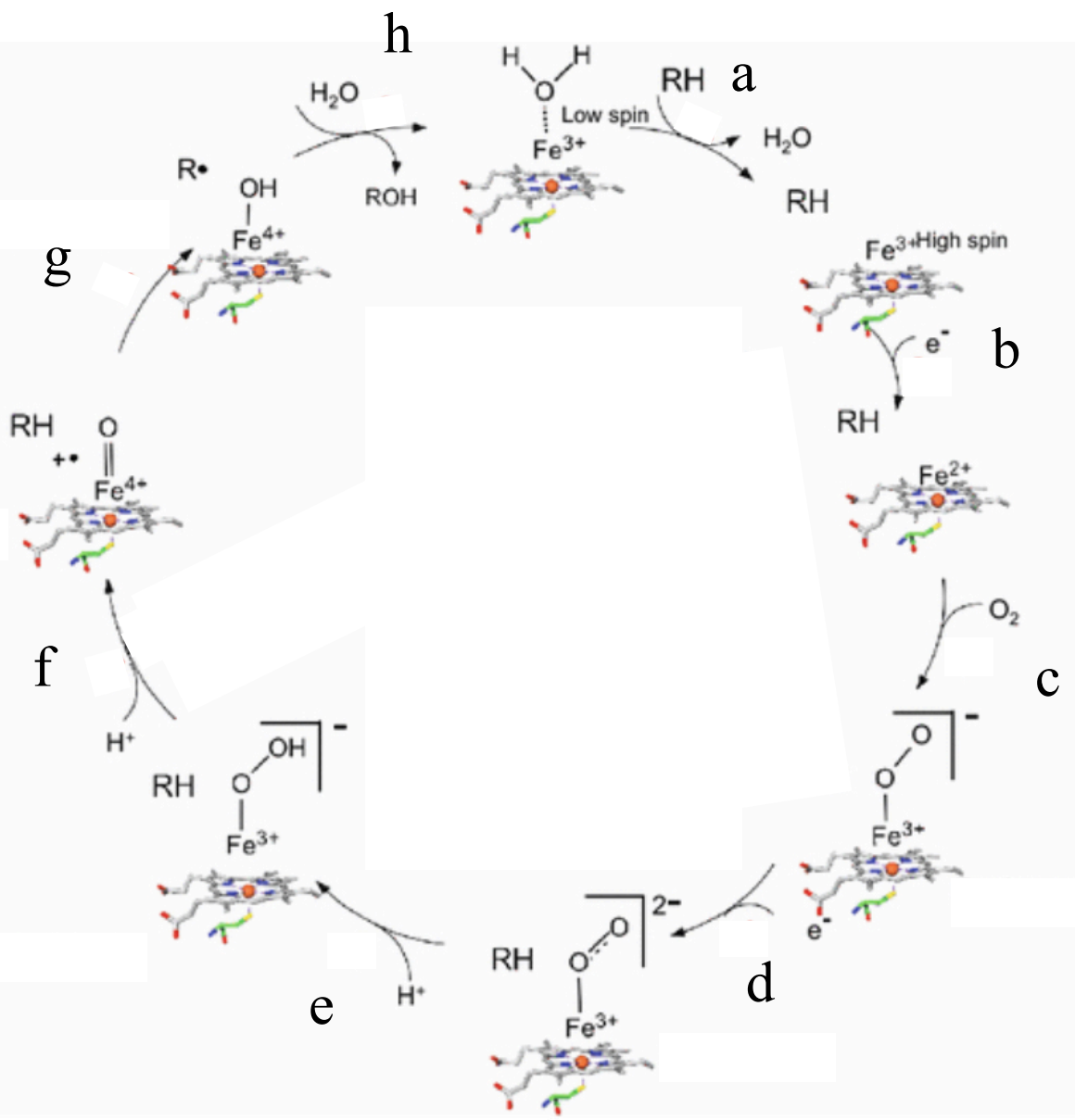

Figure 7: Catalytic cycle of a generic CYP enzyme. ${ }^{51}$ (Adapted from Gilardi and Nardo)

The 2D6 isozyme is responsible for the metabolism of approximately $25 \%$ of drugs prescribed such as antidepressants, antipsychotics, and antiarrhythmic, and is highly polymorphic within humans. ${ }^{52}$ Regarding opioids, CYP2D6 has been implicated in interactions with codeine, ethyl morphine, hydrocodone, hydromorphone, methadone, morphine, oxycodone, and oxymorphone. ${ }^{53}$ 
Part II: Electrochemical Analysis of Fentanyl

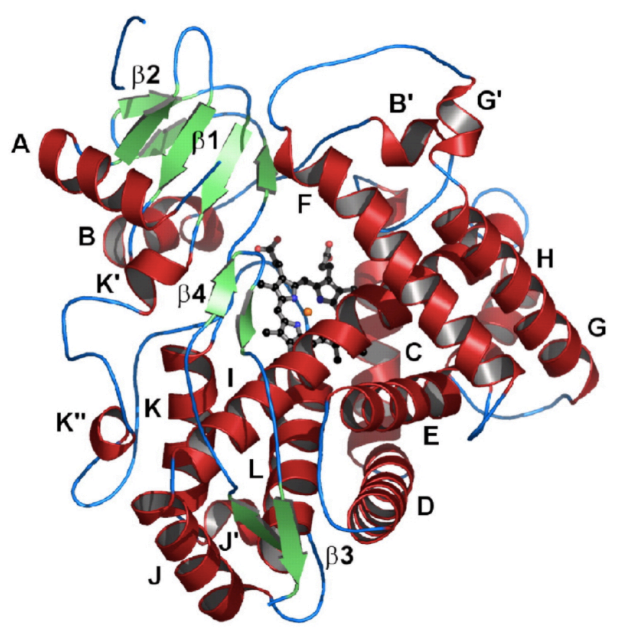

Figure 8: CYP2D6 enzyme ribbon diagram. ${ }^{54}$

The 3A4 isozyme is the most prevalent CYP enzyme in the liver (39\%) and within the small intestine (82\%). ${ }^{55}$ This enzyme metabolizes over 120 medications on the market. ${ }^{48}$ In terms of opioids, CYP3A4 has been implicated in interactions with alfentanil, buprenorphine, codeine, dihydrocodeine, fentanyl, hydrocodone, methadone, oxycodone, and sufentanil. ${ }^{56}$

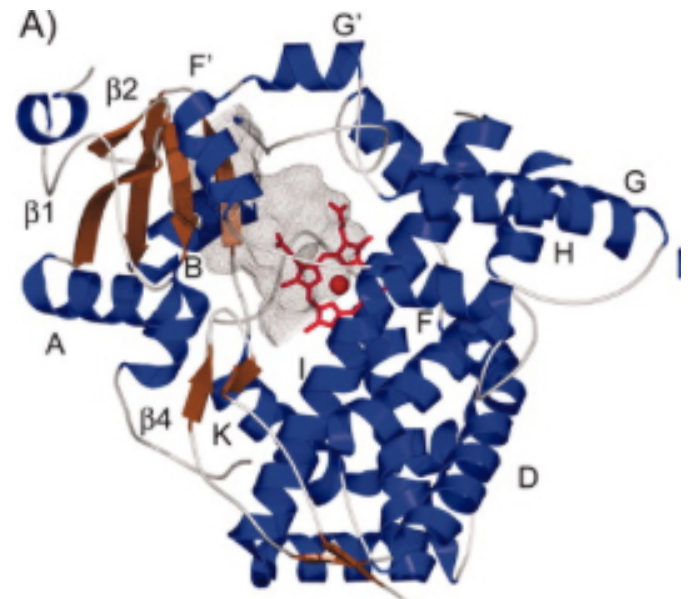

Figure 9: CYP3A4 enzyme ribbon diagram. ${ }^{57}$

\subsection{Nanomaterials and Electrode Modifications}

The field involving nanotechnology and nanoscale modifications has grown extensively, and this includes the use of nanomaterial modifications in electrochemistry. Electrochemistry has 
seen a wide variety of nanomaterial modifications including metal nanoparticles (NPs), carbonbased materials such as carbon nanotubes, enzymes, and self-assembled monolayers. These many modification techniques have been explored due to the ability of nanomaterials to improve response, biocompatibility, conductivity, and catalytic activity, ${ }^{43}$ as well as, the ability to control the size and structure..$^{58,59}$

Nanoparticles are generally considered to be between 1 and $100 \mathrm{~nm}$ particles. ${ }^{42}$ Metal nanoparticles are commonly encountered modification techniques in electrochemistry for many reasons. Metal NPs may be used as a method for enhancing the sensitivity of the detection method as they can provide bonding sites for some analytes ${ }^{60}$ and improve the electron transfer process. ${ }^{58}$ Furthermore, the use of nanoparticles on the surface of electrodes can significantly increase the surface area ${ }^{42,61}$ and improve interactions with enzymes. ${ }^{62}$ Metal NPs are also generally low cost, ${ }^{42}$ easy to immobilize on the surface of electrodes, and have high strength and chemical stability. ${ }^{63}$ Several different metals have been used for the development of nanoparticle and metal modifications including gold, silver, platinum, rhodium, and palladium. ${ }^{64}$

Other commonly encountered nanomaterials are carbon-based, such as single-walled carbon nanotubes (SWCNTs) and multi-walled carbon nanotubes (MWCNTs), as well as, graphene. These carbon structures boast high surface-area-to-volume ratios, demonstrate excellent electron transport properties over distances, ${ }^{58}$ and may act as semi-conductors or metallic conductors due to $\mathrm{sp}^{2}$ hybridization. ${ }^{41}$ SWCNTs can be thought of as a single tube-like structure formed by graphene sheets, ${ }^{41}$ while MWCNTs are an array of these structures. ${ }^{58}$ The drawbacks of using carbon nanotubes include some difficulty in manipulation and functionalization. ${ }^{41}$

Another frequently utilized modification technique is the production of self-assembled monolayers (SAMs). The technique of using SAMs involves the generation of some molecular layer forming on the surface of the electrode. The formation of the monolayers can be achieved on a variety of electrode surfaces and provide a variety of functional groups based on the SAM molecule, such as $-\mathrm{CN},-\mathrm{NH}_{2}$, and $-\mathrm{SH} .{ }^{41}$ The use of SAMs can be tailored to the required need and use single, multiple, or different sized molecules to achieve the desired effect and attempt to minimize possible problems due to the typically encountered dense monolayer formation. ${ }^{41}$

Many other electrode modification techniques are available and even more combinations of the above-mentioned modifications. However, these are outside the scope of this thesis. 


\subsection{Method Validation for Qualitative Identification and Screening}

The ASB has designated method validation guidelines concerning forensic toxicology methods. Validation ensures that a method meets the specified criteria for acceptance according to its fit for purpose. The parameters designated by ASB for validation provide an objective approach to evaluating a methodology. ${ }^{65}$ The purpose of developing the electrochemical detection method for fentanyl in this study was to provide the qualitative identification of fentanyl along with quasiquantitative data. The ASB has denoted guidelines based on the intent of the method under question (i.e., screening, qualitative, or quantitative). The required validation parameters for screening and qualitative methods are considered to be the limit of detection, interference studies, and carryover. When applicable ionization suppression/enhancement, dilution integrity, and processed sample stability should also be assessed. ${ }^{65}$

\subsubsection{Carryover and Interference Studies}

Carryover refers to a previous sample affecting the analysis of a subsequent sample. Due to the nature of the electrochemical measurements made in this study and the use of disposable screen-printed electrodes, the assessment of carryover was not performed. However, interference studies refer to the effects of matrix and other analytes on the detection capability and reliability of the method. Interferences must not affect the ability of the method to reliably identify the analyte of interest. ASB recommends that commonly encountered drugs be assessed when performing interference studies, as well as, drugs that are similar to the analyte. ${ }^{65}$

\subsubsection{Limit of Detection (LOD)}

ASB Standard 036 requires the assessment of the LOD for all types of methods and provides multiple approaches for its estimation. ${ }^{65}$ Two forms of LOD estimation are acceptable for the electrochemical analysis carried out in this study: estimation using the lowest non-zero calibrator and estimation using a linear calibration curve. Both methods imply the analysis of calibration curves by the methodology. The former may serve as a more conservative approach to 
determining LOD of a qualitative method as a positive signal must be seen and tested, while the latter is useful to assess LOD without the need to test the LOD value directly. 
Part II: Electrochemical Analysis of Fentanyl

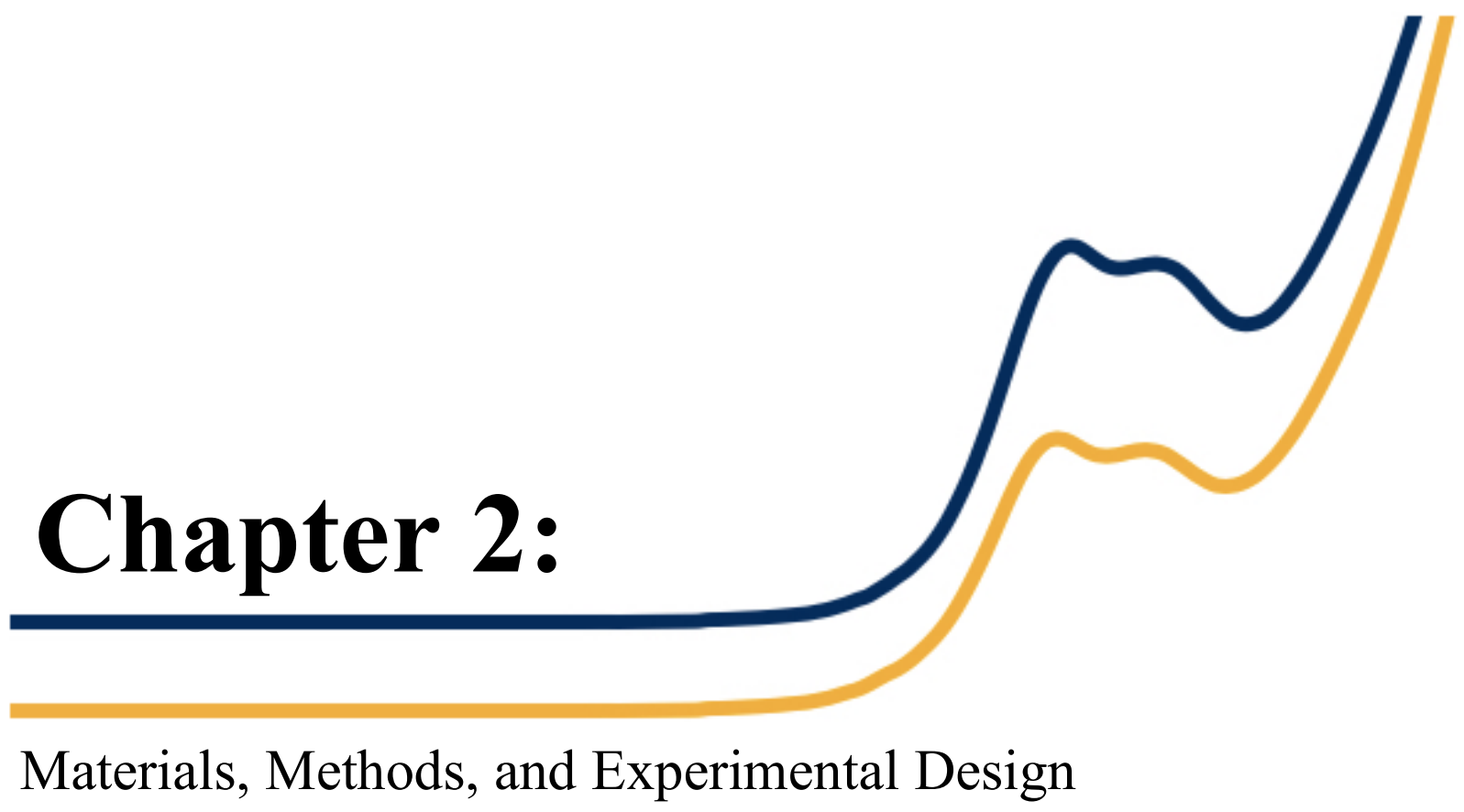




\subsection{Materials}

Fentanyl citrate was purchased from Cayman Chemical Company (Ann Arbor, MI). Acetaminophen was purchased from Sigma-Aldrich (St. Louis, MO). Methamphetamine, cocaine hydrochloride, quinine, acetaminophen, and caffeine were used for interference studies. Methanol $\left(\right.$ Optima $\left.{ }^{\circledR}\right)$ and concentrated Hydrochloric acid (Trace grade) were purchased from Fisher Scientific (Fair Lawn, NJ). Tris(hydroxymethyl)amino-methane, Monobasic sodium phosphate, dibasic sodium phosphate, sodium hydroxide, and potassium chloride were purchased from SigmaAldrich (St. Louis, MO). A Millipore Direct-Q ${ }^{\circledR}$ UV water purification system (Billerica, MA) was used to obtain purified water $(18.2 \mathrm{M} \Omega)$.

Screen-printed carbon electrodes were obtained from the Universidad de Burgos analytical chemistry department. SPCEs were fabricated using a DEK 248 screen-printing system (DEK, Weymouth, UK) with polyester screens with stencils. SPCEs contained conductive silver tracks, $\mathrm{Ag} / \mathrm{AgCl}$ pseudo-reference electrode, and carbon working $\left(0.126 \mathrm{~cm}^{2}\right)$ and counter electrodes.

\subsection{Instrumentation}

Electrochemical measurements were acquired using the PalmSens3 and PalmSens4 potentiostats with PSTrace software (Randhoeve, Netherlands). The $\mathrm{pH}$ of buffer solutions was determined using a FiveEasy Plus pH meter by Mettler-Toledo (Columbus, $\mathrm{OH}$ ).

\subsection{Preparation of Standard Working Solution}

A Fentanyl stock solution was prepared from fentanyl citrate for a final concentration of $3182 \mu \mathrm{g} / \mathrm{mL}$ for use in preparing subsequent concentrations for electrochemical analysis.

\subsection{Manufacture of Screen-Printed Carbon Electrodes}

SPCEs were provided by our colleagues in Burgos, Spain. A DEK 248 screen-printing system (Weymouth, UK) was used to manufacture the SPCEs. The sequential deposition of the appropriate inks was performed using a series of polyester screen stencils. The following layers 
were prepared: silver conductive tracks, $\mathrm{Ag} / \mathrm{AgCl}$ pseudo-reference electrode, carbon counter electrode, carbon working electrode $\left(0.126 \mathrm{~cm}^{2}\right)$, and an insulating layer.

\subsection{Electrochemical Measurements}

Voltammetric measurements were performed in phosphate-buffered saline $(50 \mathrm{mM})$ supplemented with $100 \mathrm{mM} \mathrm{KCl}$ or $\mathrm{NaNO}_{3}$ (PBS) and Tris- $\mathrm{HCl}$ (100 mM pH 8.5) as supporting electrolytes.

Cyclic voltammetry (CV) was performed to determine the electroactivity of fentanyl in PBS by analyzing increasing concentrations of fentanyl in a $5 \mathrm{~mL}$ electrochemical cell containing PBS pH 7.4 supplemented with chloride and another supplemented with nitrate. Voltammetric measurements were carried out between $-0.5 \mathrm{~V}$ and $1.6 \mathrm{~V}$ (vs. SPE $\mathrm{Ag} / \mathrm{AgCl}$ ).

Parameter optimization was conducted for $\mathrm{pH}$, supporting electrolyte, technique parameters, stirred equilibration time, and amperometric pre-treatment. Fentanyl was prepared to a concentration of $152 \mu \mathrm{g} / \mathrm{mL}$ in $\mathrm{pH} 5.5,6.0,6.5,7.0,7.4,8.0,8.5$, and 9.0. The above $\mathrm{CV}$ parameters were used to analyze a $100 \mu \mathrm{L}$ drop of each solution on the SPCE surface. Peak currents were monitored for oxidation peak I $(0.75 \mathrm{~V})$ and peak II $(0.88 \mathrm{~V})$. Square-wave voltammetry and differential pulse voltammetry were tested. Technique parameters were optimized to provide a maximum current response. PBS $(\mathrm{pH}$ 8.5) and Tris- $\mathrm{HCl}(\mathrm{pH}$ 8.5) were analyzed in the electrochemical cell through increasing concentrations of fentanyl to determine the optimal supporting electrolyte. The effect of equilibration time on current response was analyzed for times $0,10,20,40,80,160,320,640$, and 1280 seconds at a fentanyl concentration of $336 \mathrm{ng} / \mathrm{mL}$ in the electrochemical cell. Finally, anodic pre-treatment of the carbon surface was assessed to improve the current response. The potentials applied ranged between 1.0 and $1.6 \mathrm{~V}$ at times between 20 and 80 seconds.

Adsorptive stripping square-wave voltammetry of fentanyl and interfering drugs was conducted through the initial application of an anodic pre-treatment at $1.5 \mathrm{~V}$ for 40 seconds in Tris- $\mathrm{HCl}$ followed by a 320 second equilibration time to allow for adsorption. Analysis of analytes was accomplished through SWV between $-0.5 \mathrm{~V}$ and $1.6 \mathrm{~V}$ with a potential step $\left(\mathrm{E}_{\text {step }}\right)$ of 0.012 $\mathrm{V}$, an amplitude of $0.075 \mathrm{~V}$, and a frequency of $100 \mathrm{~Hz}$ either in a $5 \mathrm{~mL}$ electrochemical cell or $100 \mu \mathrm{L}$ drop. An equilibration time of 320 seconds was used for analysis. To this end, increasing 
concentrations of analyte were used for analysis in the cell, and individual solutions were used for analysis in drop. Calibration curves were constructed and assessed for linearity and reproducibility through relative standard deviation (RSD).

Interference studies were conducted on methamphetamine, caffeine, cocaine, acetaminophen, and quinine. The standard addition method with SWV procedure was used for the cell to determine any interfering species. Methamphetamine was analyzed between $149 \mathrm{ng} / \mathrm{mL}$ and $33.5 \mu \mathrm{g} / \mathrm{mL}$, caffeine between $193 \mathrm{ng} / \mathrm{mL}$ and $50.1 \mu \mathrm{g} / \mathrm{mL}$, cocaine between $303 \mathrm{ng} / \mathrm{mL}$ and 13.4 $\mu \mathrm{g} / \mathrm{mL}$, acetaminophen between $151 \mathrm{ng} / \mathrm{mL}$ and $13.8 \mu \mathrm{g} / \mathrm{mL}$, and quinine between $99 \mathrm{ng} / \mathrm{mL}$ and $9 \mu \mathrm{g} / \mathrm{mL}$. Fentanyl mixtures with cocaine, quinine, and acetaminophen were assessed for the following ratios: 0.3:10 $\mu \mathrm{g} / \mathrm{mL}, 1: 10 \mu \mathrm{g} / \mathrm{mL}$, and 1:3 $\mu \mathrm{g} / \mathrm{mL}$. A mixture between fentanyl and methamphetamine was assessed for the following ratios: $0.3: 10 \mu \mathrm{g} / \mathrm{mL}, 0.3: 20 \mu \mathrm{g} / \mathrm{mL}$, and 1:3 $\mu \mathrm{g} / \mathrm{mL}$.

An anodic stripping voltammetric approach was performed using an anodic preconcentration step, followed by the optimized square-wave procedure. Pre-concentration was achieved through the application of $0.1 \mathrm{~V}$ for 120 seconds. These parameters were optimized by varying the times and potentials to assess peak current and peak shape. During pre-concentration, the solution was stirred when performed in a cell with Tris-HCl. Prior to the application of the SWV method, the stirring was turned off to allow for a quiescent solution during SWV. Calibration curves were constructed utilizing this method and assessed for linearity and reproducibility.

\subsection{Validation}

Validation was achieved through the completion of the above-mentioned tasks to determine the limits of detection and any interfering compounds that could affect detection. The limit of detection was calculated based on the mean and the standard deviation of the y-intercept through the application of equation 4:

$$
L O D=3 \times \frac{s_{y}}{m}
$$

where $S_{y}$ is the standard deviation of the y-intercept and $m$ is the average slope. 


\subsection{Electrode Modification Techniques}

The following modification techniques were performed on SPCEs for an investigation into their ability to improve the signal for fentanyl in order to achieve lower limits of detection.

\subsubsection{Silver Nanoparticles}

Two varying concentrations of silver nitrate were investigated for use: $0.1 \mathrm{mM}$ silver nitrate and $5 \mathrm{mM}$ silver nitrate. Concentrations were prepared in Britton-Robinson buffer $\mathrm{pH}$ 2.0. Deposition of the nanoparticles on the carbon working electrode was achieved through the application of $-0.12 \mathrm{~V}$ for varying amounts of time to control the deposition process. The silver solution was stored protected from light.

\subsubsection{Gold Nanoparticles}

Gold chloride trihydrate was used to make $1 \mathrm{mM}$ solutions in $0.5 \mathrm{M}$ sulfuric acid. Deposition of gold nanoparticles onto the carbon working electrode was achieved through amperometric means by the application of $0.18 \mathrm{~V}$. Deposition of gold nanoparticles onto a gold working electrode surface was achieved through the amperometric application of $-0.4 \mathrm{~V}$. In both instances the deposition time was varied to control the amount of gold deposited onto the surface of the electrode.

\subsubsection{Rhodium Nanoparticles}

Rhodium nanoparticles were deposited onto the carbon working electrode surface using a solution of $0.1 \mathrm{mM}$ Rhodium (III) chloride crystals in $0.5 \mathrm{M}$ sulfuric acid. The deposition was achieved through the application of $-0.25 \mathrm{~V}$ for 480 seconds. Again, a variation of both the time and potential can be used to control the deposition of the nanoparticles. 


\subsubsection{Multiwalled Carbon Nanotubes}

A solution of $3 \mathrm{mg} / \mathrm{mL}$ of MWCNTs in DMF was prepared. The solution was allowed to sonicate for approximately 1 hour to remove aggregates. A small volume of the solution was placed onto the working electrode, being careful to keep the solution only on the w.e., and then allowed to dry before use.

\subsubsection{Graphene Oxide}

The desired concentration of graphene oxide was prepared from an aqueous solution of graphene oxide. This solution was placed on the working electrode and allowed to dry. Reduction of the graphene oxide surface was achieved by cyclic voltammetry in $0.1 \mathrm{M}$ potassium nitrate between $-0.2 \mathrm{~V}$ and $-1.5 \mathrm{~V}$ with a potential step of $0.003 \mathrm{~V}$, a scan rate of $0.05 \mathrm{~V} / \mathrm{sec}$, and 5 scans. 
Part II: Electrochemical Analysis of Fentanyl

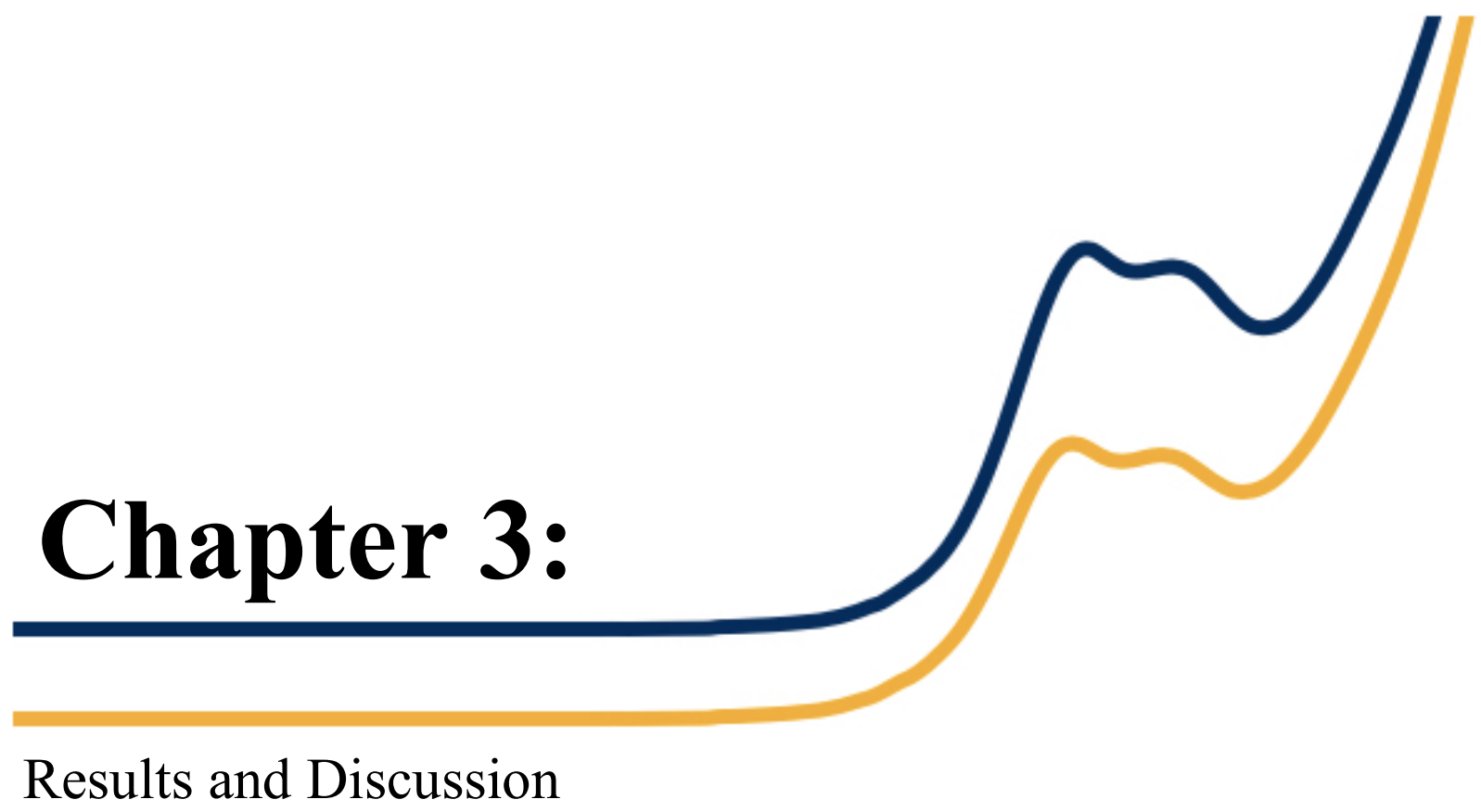




\subsection{Electrochemical Behavior of Fentanyl}

In order to demonstrate the electroactivity of fentanyl within a cell, cyclic voltammetry was performed between $-0.5 \mathrm{~V}$ and $1.6 \mathrm{~V}$. Cyclic voltammetry of fentanyl in PBS buffer demonstrated a broad oxidative peak between $0.8 \mathrm{~V}$ and $1.2 \mathrm{~V}$ (Figure 10). The lack of reduction peaks suggests that there was a non-reversible electron transfer taking place. As the fentanyl concentration was increased, it became apparent that two oxidative peaks were present, possibly suggesting two separate electron transfer steps taking place in the redox process of fentanyl. These two peaks became better resolved with higher concentration. Calibration curves were constructed from the CV experiments in PBS supplemented with chloride and PBS supplemented with nitrate. Figure 10 demonstrates a calibration curve constructed with the supporting electrolyte as PBS with chloride ion. Excellent linearity was observed for analysis of fentanyl in both buffers, with correlation coefficients above 0.99. Further analysis was focused on improving response to fentanyl in order to resolve these peaks and achieve lower detection capability.
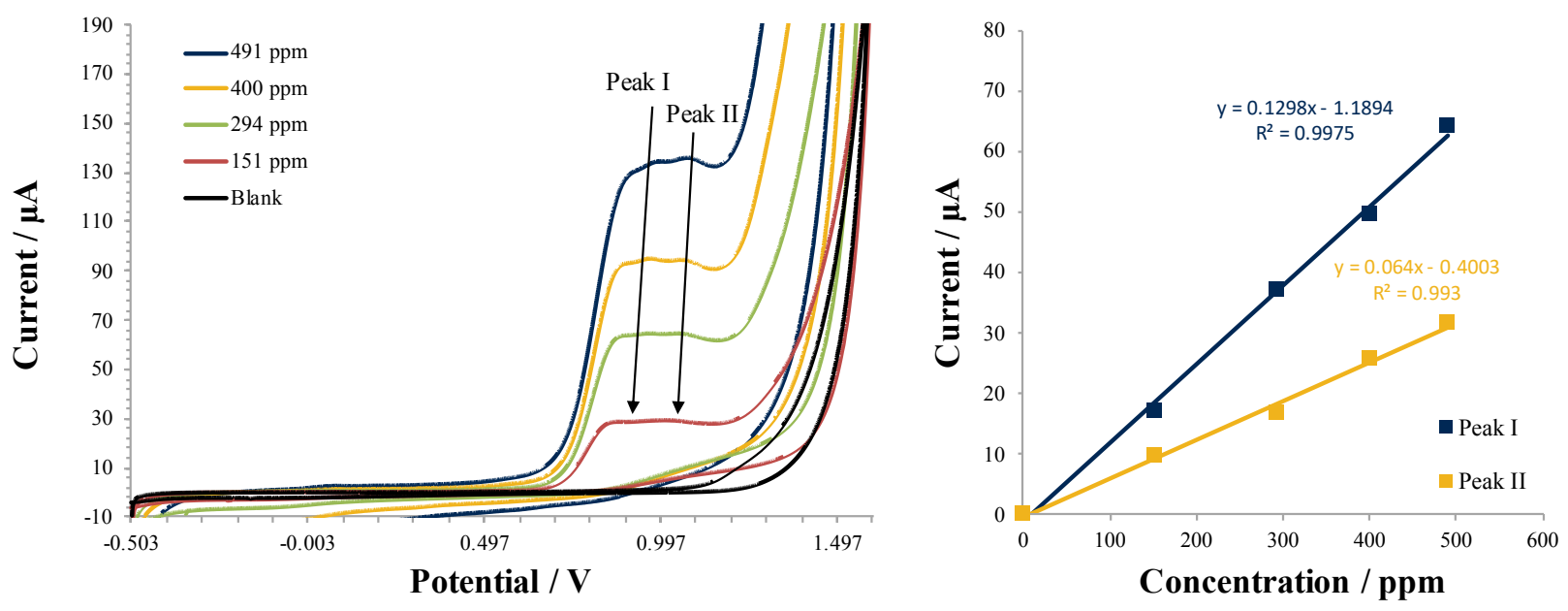

Figure 10: Cyclic voltammetry of fentanyl at an SPCE in a $5 \mathrm{~mL}$ cell containing PBS buffer pH 7.4 (left) and resulting calibration curve for $C V$ performed in $P B S$ supplemented with chloride.

A solution of citrate was prepared due to the fentanyl being present with citrate in the standard solution. Increasing concentrations of analyte solution were used to assess any electroactivity due to citrate using cyclic voltammetry. There was no current response for citrate concentrations between 0 and $1.46 \mathrm{mM}$ (Figure 11). 
Part II: Electrochemical Analysis of Fentanyl

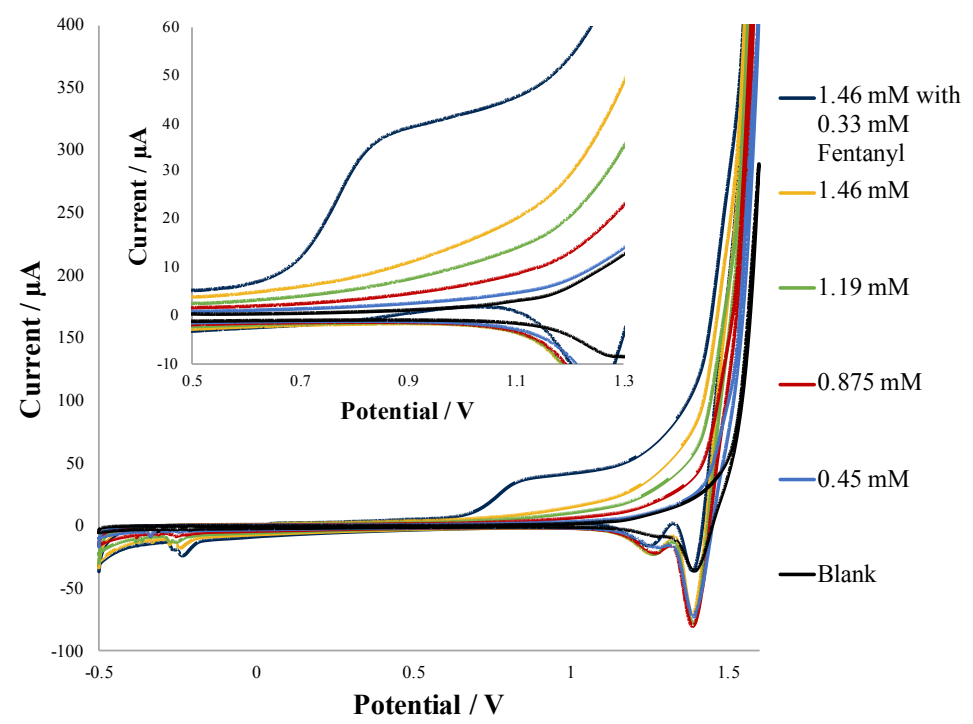

Figure 11: Cyclic voltammetry of citrate in PBS buffer supplemented with chloride and nitrate demonstrating no response to citrate and response to only fentanyl.

\subsection{Optimization of Parameters and Techniques}

Optimization of the $\mathrm{pH}$ of the supporting electrolyte demonstrated maximum current response at a $\mathrm{pH}$ of 8.5 (Figures 12 and 13). Current response peaked at this $\mathrm{pH}$ value and immediately began to decrease at more basic $\mathrm{pH}$ values. Optimization of the $\mathrm{pH}$ proved crucial for the improvement of the current response to fentanyl. This improvement in detection capability may be due to the closeness of the buffer $\mathrm{pH}$ with the $\mathrm{pK}$ a of fentanyl, which is $8.4 .^{26}$ This may allow for easier electron transfer with the molecule and electrode.

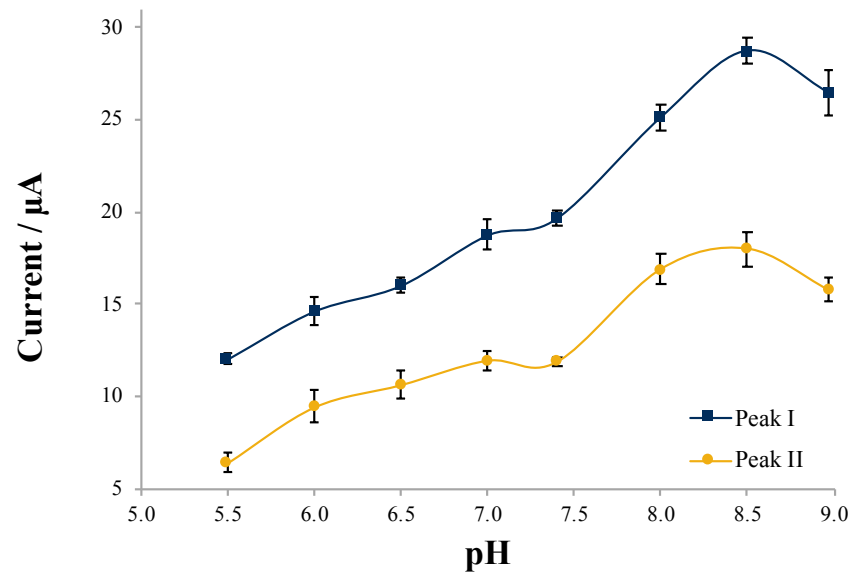

Figure 12: Current response to $151 \mu \mathrm{g} / \mathrm{mL}$ fentanyl at differing $p H$ values. Three replicates were performed per $p H$ value. The analysis was performed in a drop on the SPCE surface. 


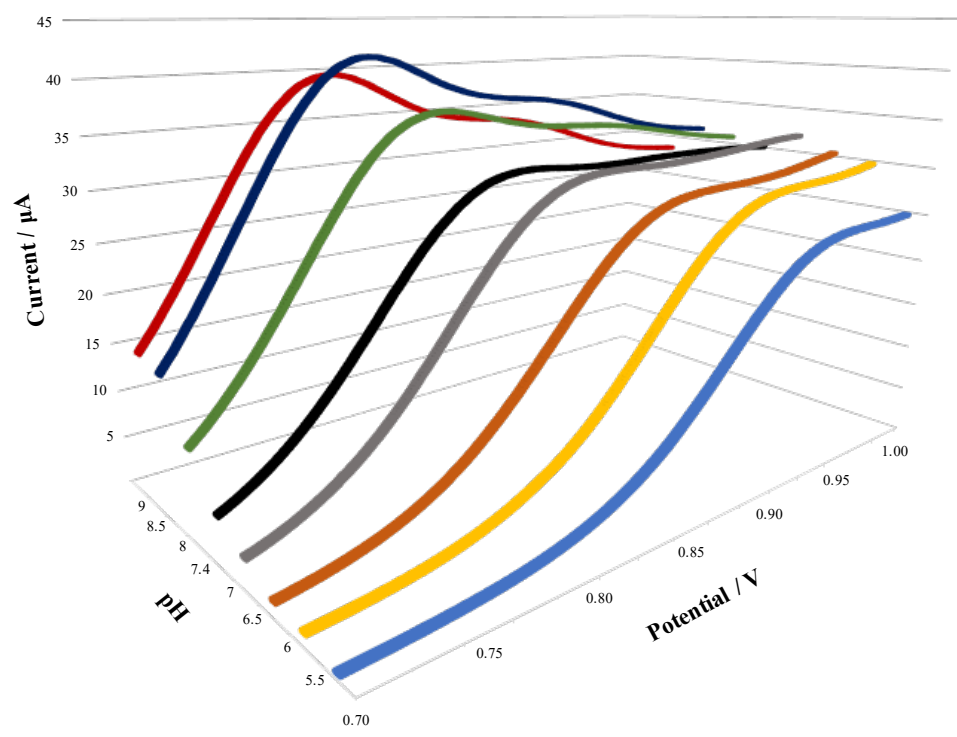

Figure 13: Demonstration of peak improvement based on buffer $\mathrm{pH}$ showing improved peak current and two-peak definition at a $\mathrm{pH}$ of 8.5 in PBS.

The optimal $\mathrm{pH}$ was tested using PBS buffer supplemented with chloride. The $\mathrm{pH}$ measurements were tested in triplicate and demonstrated good reproducibility throughout the range, with relative standard deviations of $2.5 \%$ for peak I and $5.3 \%$ for peak II at a $\mathrm{pH}$ of 8.5 (Table 2).

Table 2: Relative standard deviation, reported as percent, for triplicate analysis of the pH range.

\begin{tabular}{ccc}
\hline & \multicolumn{2}{c}{$\%$ RSD } \\
\cline { 2 - 3 } $\mathrm{pH}$ & Peak I & Peak II \\
\hline 5.50 & 2.6 & 7.4 \\
6.00 & 5.1 & 9.4 \\
6.50 & 2.8 & 6.8 \\
7.00 & 4.4 & 4.4 \\
7.40 & 1.8 & 2.0 \\
8.00 & 2.8 & 4.8 \\
8.50 & 2.5 & 5.3 \\
8.97 & 4.6 & 4.1 \\
\hline Average: & 3.3 & 5.5
\end{tabular}

The optimized $\mathrm{pH}$ was used to assess which electrochemical technique would prove most useful for analysis. Due to their high sensitivity in detecting low concentrations of analytes, squarewave voltammetry and differential pulse voltammetry were explored. Through the analysis of a single concentration of fentanyl, $151 \mu \mathrm{g} / \mathrm{mL}$, the parameters for both methods were optimized through the varying of technique parameters by monitoring peak current response for both peaks 
I and II. For SWV, the potential step ( $\left.E_{\text {step }}\right)$, amplitude, and frequency were varied for a scan between $-0.5 \mathrm{~V}$ and $1.6 \mathrm{~V}$. The optimal SWV parameters were determined to be a potential step of $0.012 \mathrm{~V}$, an amplitude of $0.075 \mathrm{~V}$, and a frequency of $100 \mathrm{~Hz}$. For DPV, the potential pulse, pulse time, and scan rate were varied for a scan between the same potential window as for SWV. The parameters chosen for DPV were a potential pulse of $0.103 \mathrm{~V}$, a pulse time of 0.01 seconds, and a scan rate of $0.6 \mathrm{~V} / \mathrm{sec}$. The optimal parameters for each method were chosen and calibration curves between $0.336 \mu \mathrm{g} / \mathrm{mL}$ and $16 \mu \mathrm{g} / \mathrm{mL}$ were constructed for each technique. Both methods appeared to perform similarly except that the DPV method was unable to see the lowest concentration. Based on calibration curves performed for the two methods, SWV resulted in a steeper slope which suggested that this method would allow for increased sensitivity, and thus, lower limits of detection for fentanyl (Figure 14). As such, SWV was chosen as the electrochemical technique for this analysis.
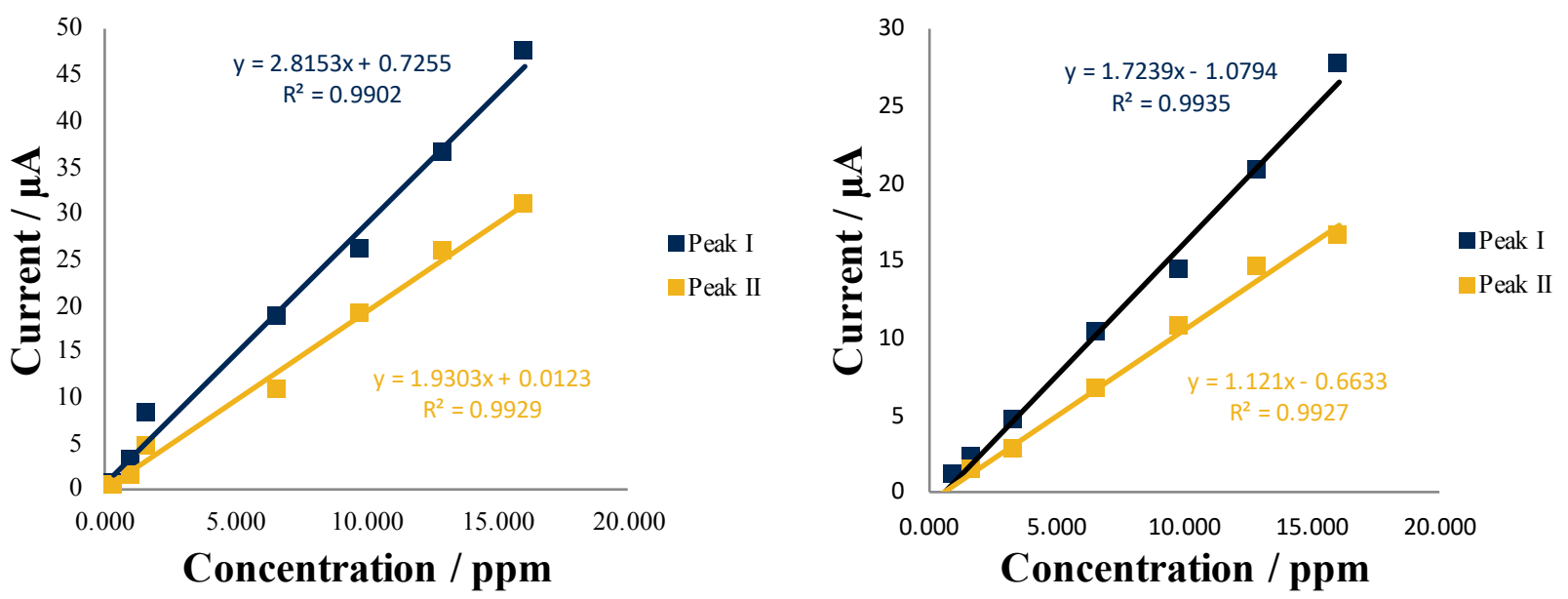

Figure 14: Comparison of calibration curves for SWV (left) and DPV (right) methods demonstrating higher sensitivity with $S W V$.

A voltammetric stripping approach was also investigated for use in conjunction with SWV through the application of varying potentials over a 10 -second interval. Calibration curves were analyzed for each of the potential changes and the resulting calibration curves were plotted. These curves generally demonstrated steeper slopes that SWV or DPV, however, linearity was slightly worse although still acceptable with average correlation coefficients greater than 0.98 . These results demonstrated that the use of stripping voltammetry might hold promise as another method for analysis of fentanyl; however, currents were similar to those produced using only SWV. 
Various buffers were explored for use as the supporting electrolyte. Comparison between $\mathrm{PBS} / \mathrm{Cl}$ and Tris-HCl, both at a $\mathrm{pH}$ of 8.5 , demonstrated higher current response in the lower concentrations of fentanyl when present in the Tris-HCl buffer. For this reason, Tris-HCl was used for further analysis, although both buffers proved to be acceptable supporting electrolyte solutions. The use of $\mathrm{NaOH}$ was also explored; however, as predicted by $\mathrm{pH}$ experiments, peaks were either absent or extremely poor. Amperometric pre-treatment of the carbon surface in Tris-HCl was explored due to observing an effect on the current response from fentanyl. After exploration of various potentials and times for pre-treatment, $1.5 \mathrm{~V}$ over 40 seconds resulted in the largest current response for both peak I and peak II at the lowest concentration. It was expected that this improvement in signal resulted from preparing the working electrode surface through the application of more positive potential than the oxidation of fentanyl. Finally, an effect in the current response was observed during the testing of equilibration time. Increasing the amount of time the solution was allowed to stir (for cell measurements) or sit on the electrode surface (for drop measurements) before analysis resulted in larger current response. Although increasing the amount of time on the electrode surface increased current response, the optimal time was chosen to be 320 seconds in order to maintain a fast analysis procedure (Figure 15). This behavior was attributed to the adsorption of fentanyl to the carbon working electrode, serving to pre-concentrate the analyte at the surface, thus improving response and appearing like an adsorptive stripping technique.
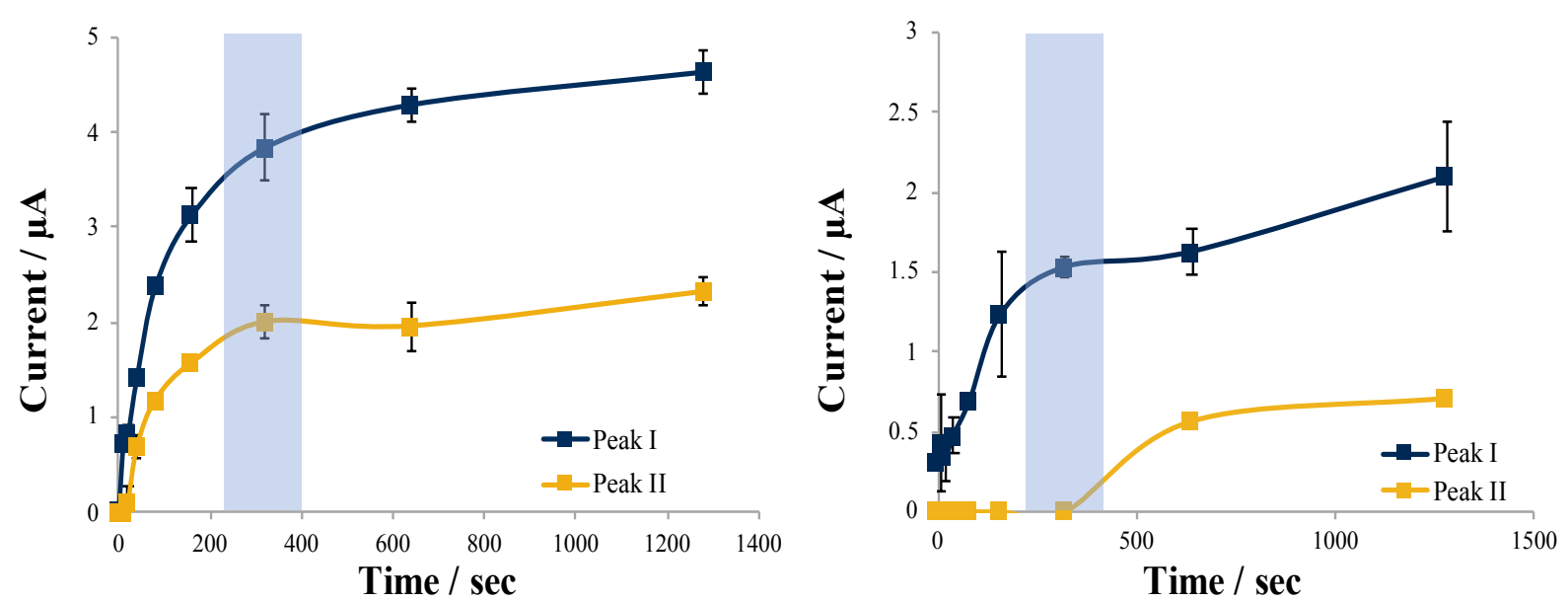

Figure 15: Stirred equilibration time optimization in a cell containing Tris-HCl at a fentanyl concentration of $336 \mathrm{ng} / \mathrm{mL}$. Three replicates per time were tested with an RSD of approximately $9 \%$ at 320 seconds (left) and stirred equilibration time in $100 \mu \mathrm{L}$ drop of Tris- $\mathrm{HCl}$ at $336 \mathrm{ng} / \mathrm{mL}$ with RSD of approximately $4.5 \%$ for two replicates at $\mathrm{pH} 8.5$ (right). 


\subsection{Electrochemical detection of fentanyl}

The optimized parameters were then used to construct calibration curves for the detection of fentanyl. The use of increasing concentrations of fentanyl to a $5 \mathrm{~mL}$ electrochemical cell was performed for analysis between $75 \mathrm{ng} / \mathrm{mL}$ and $6.88 \mu \mathrm{g} / \mathrm{mL}$. Fentanyl was able to be detected for the lowest concentration tested, $75 \mathrm{ng} / \mathrm{mL}$. Figure 5 demonstrates the improved resolution of the two oxidation peaks present and shows a small positive potential shift with increasing concentration. Peak I appeared at approximately $0.75 \mathrm{~V}$ and peak II appeared at approximately $0.88 \mathrm{~V}$. Construction of calibration curves for peak I and peak II demonstrated two areas of linearity, one for concentrations between $75 \mathrm{ng} / \mathrm{mL}$ to $637 \mathrm{ng} / \mathrm{mL}$ and the second between 1.31 $\mu \mathrm{g} / \mathrm{mL}$ to $6.88 \mu \mathrm{g} / \mathrm{mL}$. Two regions of linearity have been previously demonstrated within the literature for detection of other substance. ${ }^{66-69}$ This phenomenon is likely due differences in the oxidation reaction kinetics as a result of working electrode size, adsorption, and diffusion to the surface of the working electrode. Three replicates were performed at each concentration for the construction of calibration curves. Linearity was achieved for oxidation peaks I and II within linear ranges, as demonstrated in Figure 16.
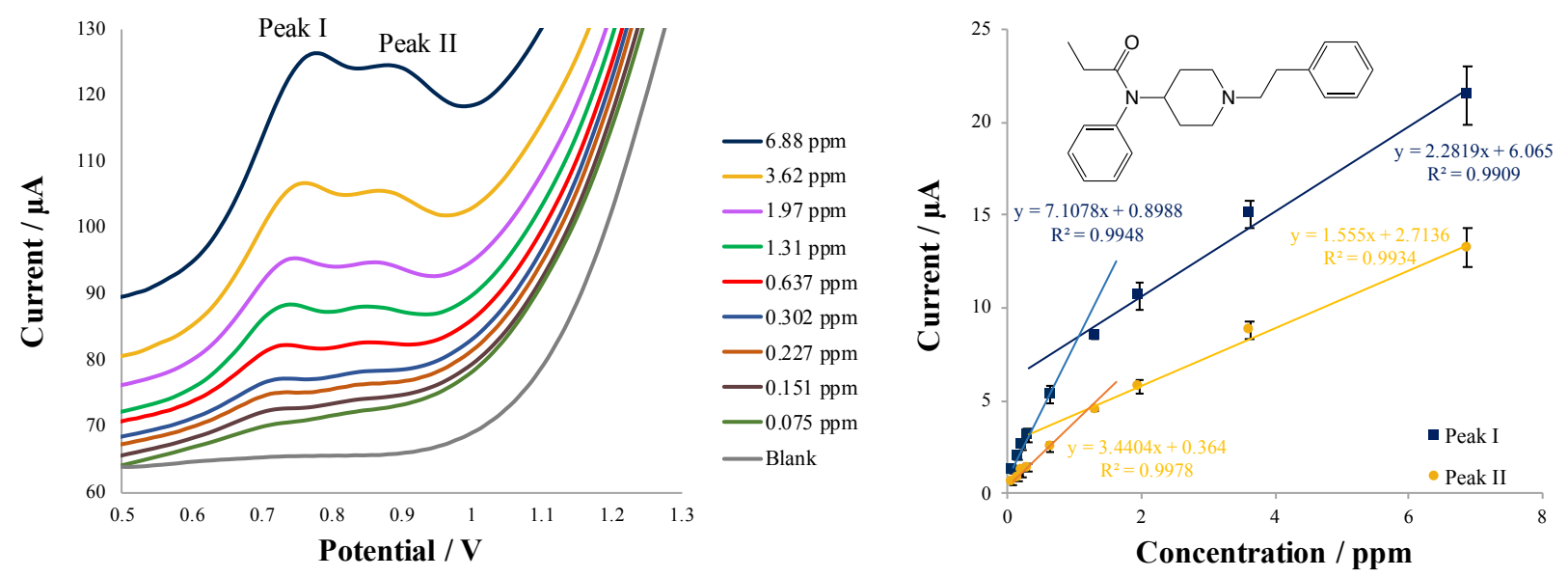

Figure 16: AdS-SWV for the standard addition of fentanyl to a $5 \mathrm{~mL}$ electrochemical cell containing 100 $m M$ Tris- $\mathrm{HCl} \mathrm{pH} 8.5$ in triplicate (left) and constructed calibration curve for peaks I and II for fentanyl (right).

Similar results were obtained for analysis of fentanyl in a 100 microliter drop. Concentrations for analysis ranged between $0.302 \mu \mathrm{g} / \mathrm{mL}$ and $6.88 \mu \mathrm{g} / \mathrm{mL}$ (Figure 17). The lowest 
concentration detected $(0.302 \mu \mathrm{g} / \mathrm{mL})$ using the drop technique was determined to be the limit of detection. Also, the drop method demonstrated a slightly noisier response; however, linearity was achieved over the concentration range (Figure 8). The correlation coefficients for peak I and peak II were 0.9977 and 0.9848 , respectively. Three replicates were performed per concentration and resulted in an average RSD of approximately $8 \%$ for peak I and peak II. The difference in the limit of detection can be attributed to solution dynamics within the two methods. When analyzed in a cell, the solution can be stirred before analysis. This mechanical action allows for the accumulation of the fentanyl via adsorption to the carbon surface and diffusion towards the working electrode. However, when the experiment is run as a drop, stirring is not possible, and the electroactivity of fentanyl may rely more on diffusion. Although, as explained before, under both the cell and drop method, both adsorption and diffusion processes are concurrently observed.
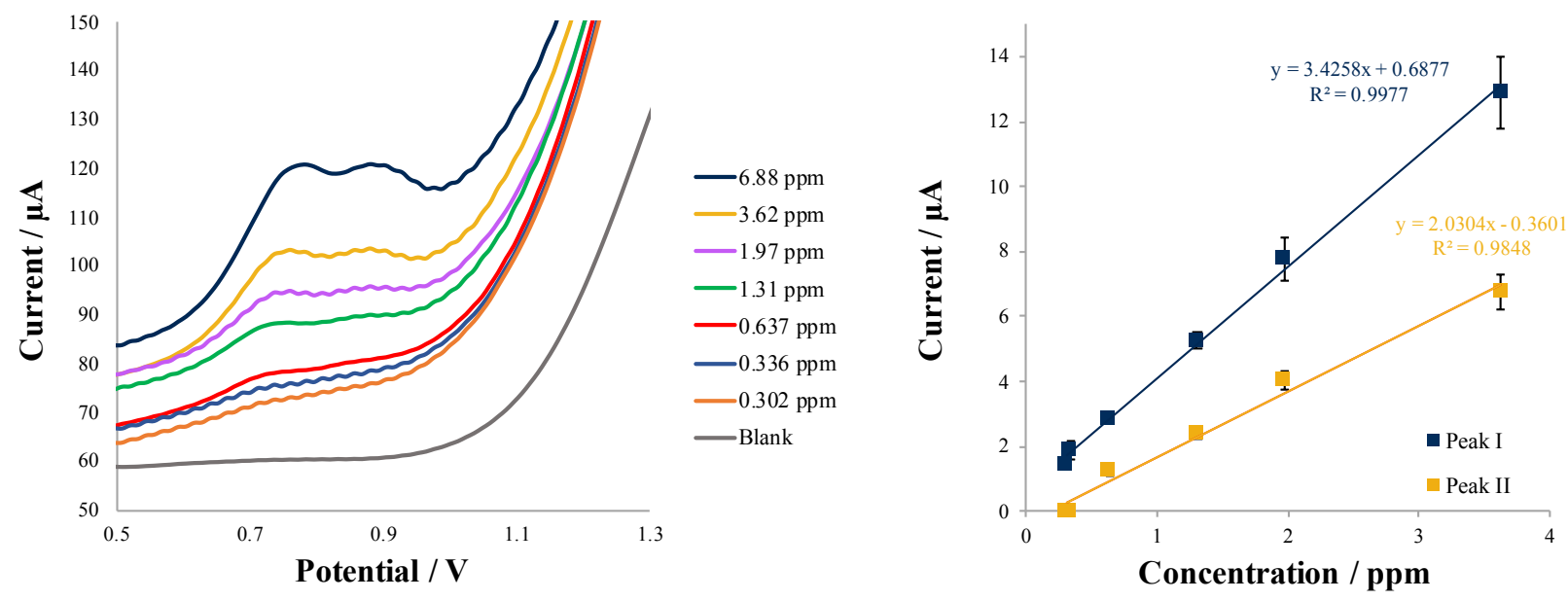

Figure 17: AdS-SWV for increasing concentrations of fentanyl in a $100 \mu \mathrm{L}$ drop of Tris-HCl on the surface of the SPCE (left) and constructed calibration curve for fentanyl assessed in triplicate (right).

Drop analysis differed from the results generated in a cell due to the presence of only a single linear calibration range as opposed to the two observed for the cell method. One explanation for this is where the limit of detection lies for the method. Since the LOD was larger when performed in a drop, the dynamic range does not extend as low as the one for the cell method. Therefore, it is possible that the differing linear range could not be observed, though it may still be present. Another explanation could be from the result of the diffusion-limited conditions. Due to the absence of stirring the solution, the working electrode surface may not have become saturated due to decreased adsorption from lack of fentanyl molecules reaching the surface. Therefore, the kinetics between the two methods could be much different. 


\subsection{Figures of Merit}

\subsubsection{Accuracy}

In order to evaluate the detection methodology chosen herein, the accuracy, precision, and limit of detection was determine using the optimized method parameters. This method was developed for qualitative identification of fentanyl in samples. As such, the accuracy of the method was determined through the analysis of interfering compounds and blank buffer. This information can be found in section 4.5 below. However, the method was assessed for use as a quasiquantitative method. To this end, samples were analyzed on different electrodes and compared to calibration curves obtained before analysis. Analysis of single-blind samples was conducted, including negatives, in a $5 \mathrm{~mL}$ cell containing Tris- $\mathrm{HCl} \mathrm{pH}$ 8.5. Negative samples were correctly identified as negative, containing no fentanyl, demonstrating a $0 \%$ false-positive rate $(\mathrm{n}=3$ for blank). Several samples were seen to have significant percent errors; however, these errors were in the larger concentrations of fentanyl while the lower concentrations demonstrated good accuracy for a presumptive identification technique (Table 3). This discrepancy between low and high concentrations could have been due to a single pre-treatment before performing calibration curves, demonstrating the increased sensitivity following the pre-treatment, which was lost in the calibration curves at higher concentrations, leading to larger currents during unknown analysis, consisting of a blank and a single measurement. In order to better observe the trend in accuracy for quasi-quantitation, more single-blind samples were prepared and subsequently analyzed by the method (Table 4). Again, the same trend was seen demonstrating better accuracy in the low concentrations compared to the high concentrations. Another reason for this discrepancy could be the effect of fentanyl adsorption on the electrode surface and saturation of the surface at higher concentrations. Furthermore, the use of a single electrode for the creation of calibration curves could have resulted in lost sensitivity from using the electrode surface multiple times. 
Table 3: Accuracy of single-blind test samples containing fentanyl ( $n=2, n=3$ for blank)

* Calculated concentration based on peak I only

$\mathrm{ND}=$ none detected

\begin{tabular}{lccccc}
\hline Sample Number & $\begin{array}{c}\text { Current Peak I } \\
(\mu \mathrm{A})\end{array}$ & $\begin{array}{c}\text { Calculated } \\
\text { Concentration } \\
(\mu \mathrm{g} / \mathrm{mL})^{*}\end{array}$ & $\begin{array}{c}\text { Prepared } \\
\text { Concentration } \\
(\mu \mathrm{g} / \mathrm{mL})\end{array}$ & $\begin{array}{c}\text { Error Peak I } \\
(\%)\end{array}$ & $\begin{array}{c}\text { RSD Peak I } \\
(\%)\end{array}$ \\
\hline Unknown 1 & 0.65 & 0.063 & 0.080 & -22 & 16 \\
Unknown 2 & 15 & 6.98 & 3.97 & 63 & 22 \\
Unknown 3 & 6.2 & 0.732 & 0.999 & -29 & 5.1 \\
Unknown 4 & 2.6 & 0.295 & 0.299 & -3.0 & 7.8 \\
Unknown 5 & $\mathrm{ND}$ & $\mathrm{ND}$ & $\mathrm{ND}$ & $\mathrm{ND}$ & $\mathrm{ND}$ \\
Unknown 6 & 1.4 & 0.164 & 0.150 & 2.4 & 15 \\
Unknown 7 & 20 & 10.8 & 5.99 & 82 & 4.1 \\
\hline
\end{tabular}

Table 4: Single-blind test sample analysis

\begin{tabular}{lccccc}
\hline Sample Number & $\begin{array}{c}\text { Current Peak I } \\
(\mu \mathrm{A})\end{array}$ & $\begin{array}{c}\text { Calculated } \\
\text { Concentration } \\
(\mu \mathrm{g} / \mathrm{mL})^{*}\end{array}$ & $\begin{array}{c}\text { Prepared } \\
\text { Concentration } \\
(\mu \mathrm{g} / \mathrm{mL})\end{array}$ & $\begin{array}{c}\text { Error Peak I } \\
(\%)\end{array}$ & \\
\hline Unknown 7 & $\mathrm{ND}$ & $\mathrm{ND}$ & 0.000 & $\mathrm{NA}$ & \\
Unknown 1 & 0.81 & 0.093 & 0.090 & 3.4 & Below LOD \\
Unknown 2 & 1.03 & 0.115 & 0.130 & -12 & \\
\hline Unknown 3 & 1.46 & 0.158 & 0.200 & -21 & Below \\
Unknown 5 & 2.94 & 0.309 & 0.330 & -6.3 & $1 \mu \mathrm{g} / \mathrm{mL}$ \\
Unknown 13 & 3.87 & 0.404 & 0.410 & -1.6 & \\
Unknown 6 & 3.67 & 0.384 & 0.500 & -23 & Above \\
Unknown 4 & 5.10 & 0.529 & 0.600 & -12 & $1 \mu \mathrm{g} / \mathrm{mL}$ \\
\hline Unknown 8 & 5.15 & 0.732 & 1.30 & -44 & \\
Unknown 11 & 6.83 & 2.34 & 2.00 & 17 & \\
Unknown 12 & 8.70 & 4.14 & 3.00 & 38 & \\
Unknown 9 & 11.4 & 6.76 & 5.00 & 35 & -11 \\
Unknown 10 & 10.0 & 5.35 & 6.00 & & \\
\hline
\end{tabular}

* Calculated concentration based on peak I only

$\mathrm{ND}=$ none detected 


\subsubsection{Reproducibility}

Reproducibility was assessed through the comparison of calibration curves generated through the standard addition method using differing electrodes. The compared calibration curves were performed with SPCEs cut from the same sheet and different sheets. The percent relative standard deviation was assessed. The average relative standard deviation for calibration curves performed in a $5 \mathrm{~mL}$ electrochemical cell was approximately $12 \%$ for peak I and approximately $18 \%$ for peak II. For the calibration curves performed as a drop on the electrode surface, the relative standard deviation was approximately $13 \%$ and $15 \%$ for peak I and peak II, respectively. It was apparent that the current response of peak I from the oxidation of fentanyl provided a more reproducible signal across electrodes and different days. Peak II was more variable, which could be expected due to the smaller size and absence of the peak for the lower concentrations.

\subsubsection{Limit of Detection}

Visual observation of the resulting voltammograms allowed peak I for the oxidation of fentanyl to be seen at approximately $75 \mathrm{ng} / \mathrm{mL}$ in cell and $300 \mathrm{ng} / \mathrm{mL}$ in drop. Three replicate calibration curves were performed to lower concentrations in the cell, and an oxidation peak for fentanyl was able to be seen at a concentration of approximately $46 \mathrm{ng} / \mathrm{mL}$ fentanyl. However, statistical analysis of calibration curves was conducted to determine the statistical limit of detection above the noise level according to:

$$
L O D=3 \times \frac{s_{y}}{m}
$$

where $S_{y}$ is the standard deviation of the y-intercept and $m$ is the average slope. Based on Equation 4, the limit of detection for analysis via the electrochemical cell method was $183 \mathrm{ng} / \mathrm{mL}$ according to peak I and $108 \mathrm{ng} / \mathrm{mL}$ for peak II, resulting in the average LOD of approximately 145 $\mathrm{ng} / \mathrm{mL}$. The same statistical calculations were performed for the drop method and resulted in an average LOD of approximately $530 \mathrm{ng} / \mathrm{mL}$. As mentioned previously, these statistical measures of the LOD proved to be higher than the visual assessment based on voltammogram peaks. This situation is likely attributed to the fact that Equation 5 takes into account the possibility for noise 
within the system resulting in the multiplication of the leading coefficient. Therefore, it is reasonable to utilize these statistical LOD values for the quasi-quantitation limits of the methods.

\subsection{Interference Studies}

As fentanyl is commonly encountered in mixtures of seized drugs, as well as being used with other drugs, ${ }^{60,70}$ interference studies were conducted in order to determine the ability to detect fentanyl in the presence of adulterants. Several possible interfering species were selected due to their frequent use as adulterants as described by professionals in the forensic chemistry and toxicology field, as well as other drugs identified as being increasingly encountered with fentanyl. These substances were the illicit drugs: cocaine and methamphetamine and the adulterants: caffeine, quinine, and acetaminophen. These substances were first characterized in the electrochemical cell environment with $100 \mathrm{mM}$ Tris- $\mathrm{HCl} \mathrm{pH} 8.5$ following the established methodology for analysis with adsorptive stripping square-wave voltammetry.

Methamphetamine was analyzed between $0.149 \mu \mathrm{g} / \mathrm{mL}$ and $33.5 \mu \mathrm{g} / \mathrm{mL}$. In general, no interfering peaks were present at potentials corresponding to fentanyl. However, in higher concentrations, the voltammogram shape began to change just after $0.9 \mathrm{~V}$. The development of a small and broad peak was observed at approximately $0.92 \mathrm{~V}$. Although there was no defined peak at interfering potentials, there was a change in shape resulting in the background current rising at earlier potentials. It was possible that this could have affected the ability to detect peak II and potentially peak I for fentanyl (Figure 18).

Caffeine was analyzed between $0.193 \mu \mathrm{g} / \mathrm{mL}$ and $50.1 \mu \mathrm{g} / \mathrm{mL}$. Larger concentrations of caffeine were analyzed due to the absence of any interfering signal at the lower concentrations. Regardless of the caffeine concentration, the shape of the voltammogram resulting from background current was unchanged and lacked any discernable peak at interfering potentials or throughout the potential range. In fact, the voltammograms were of the opposite concavity, concave up, further demonstrating the absence of any peaks (Figure 18). 
Part II: Electrochemical Analysis of Fentanyl
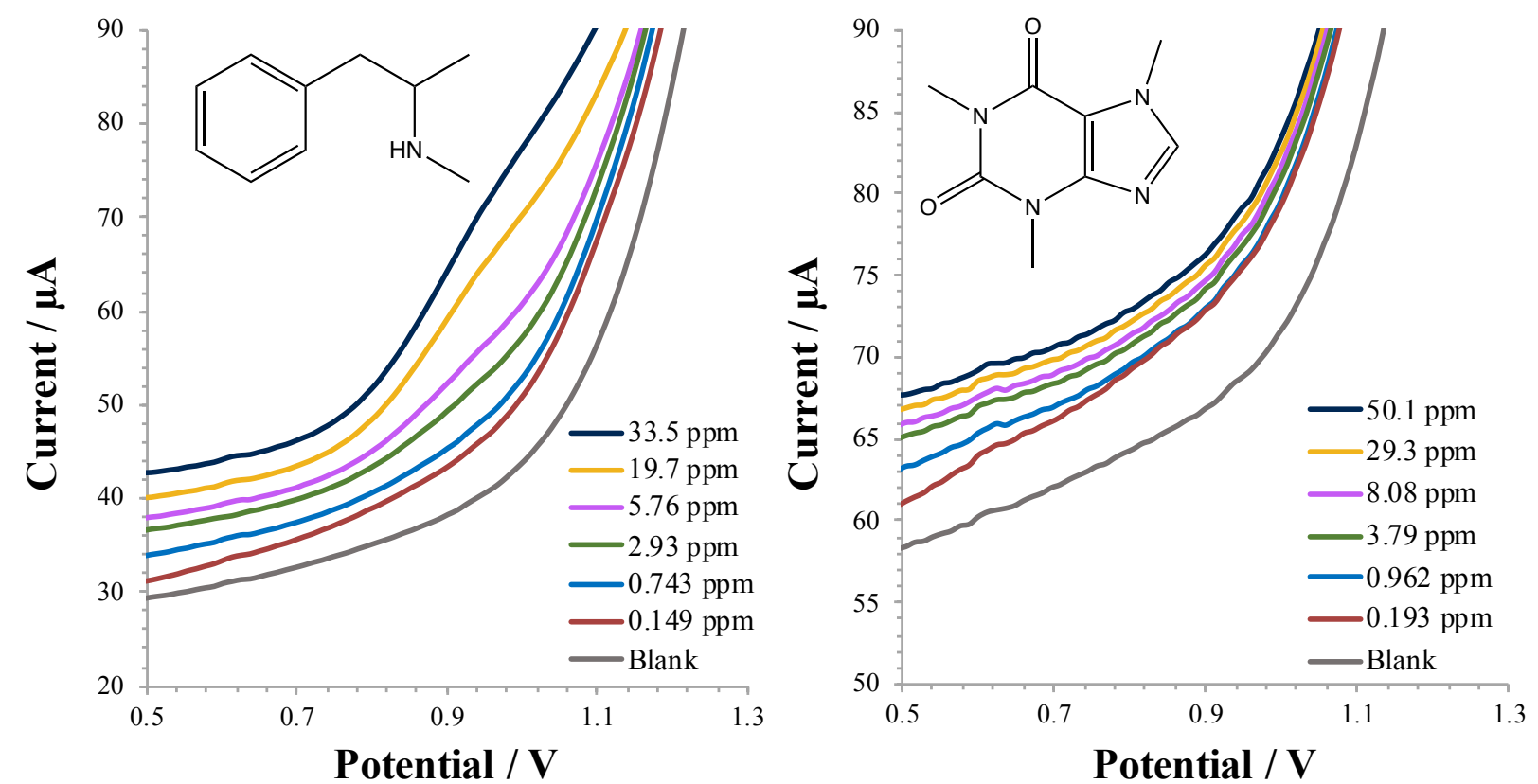

Figure 18: Adsorptive stripping square-wave voltammograms for the standard addition of methamphetamine (left) and caffeine (right) in a $5 \mathrm{~mL}$ electrochemical cell containing $100 \mathrm{mM}$ Tris- $\mathrm{HCl}$ pH 8.5.

An investigation into the effect of the change in the shape of the voltammogram at large concentrations of methamphetamine was conducted to determine if there would be an influence on the ability to detect fentanyl. Various ratios between fentanyl and methamphetamine were investigated. At a ratio of 0.3:20 (fentanyl:methamphetamine), fentanyl peak I was readily apparent, as was a small peak around $0.9 \mathrm{~V}$. This second peak could be attributed to the mixture between methamphetamine and fentanyl, resulting in only peak I being used for the identification of fentanyl. At a ratio of $0.3: 10$, a similar occurrence was noticed; however, peak II appeared to be slightly better resolved, although the peak was still small and broad. At a ratio of 1:10, both fentanyl oxidation peaks were apparent at their respective potentials, allowing for identification of fentanyl-based on both peaks (Figure 19). 

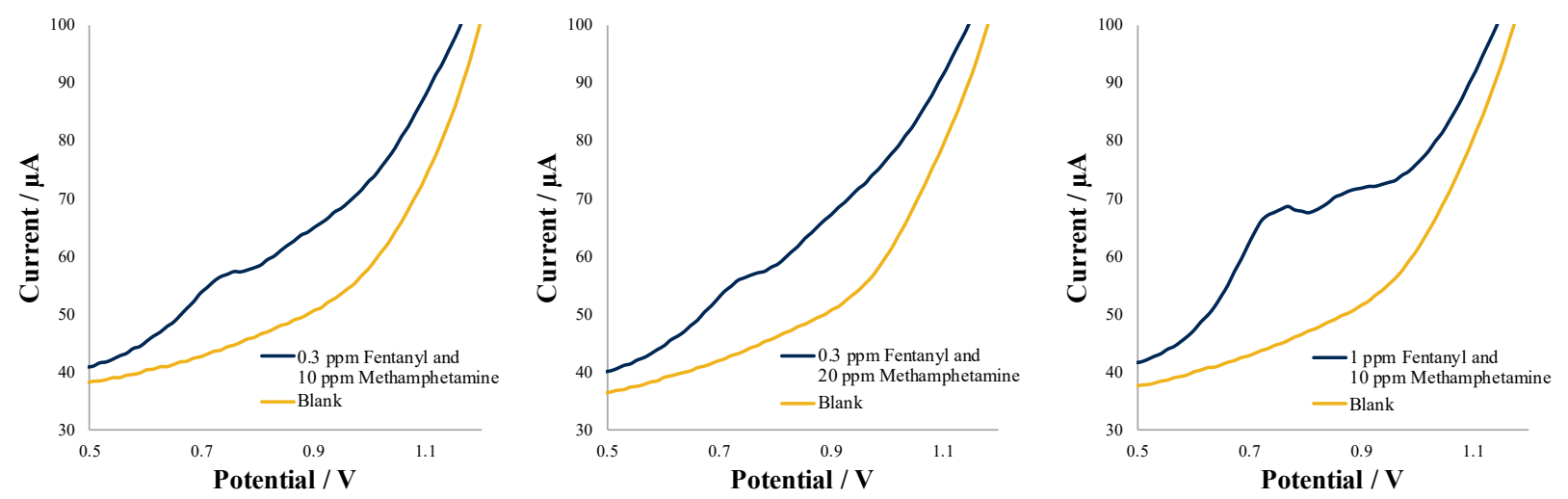

Figure 19: Adsorptive stripping square-wave voltammograms in a $5 \mathrm{~mL}$ cell with $100 \mathrm{mM}$ Tris- $\mathrm{HCl} \mathrm{pH} 8.5$ for the analysis of fentanyl in the presence of methamphetamine at ratios of 0.3:10 (left), 0.3:20 (middle), and 1:10 (right), respectively.

Cocaine was analyzed between $0.303 \mu \mathrm{g} / \mathrm{mL}$ and $13.4 \mu \mathrm{g} / \mathrm{mL}$. The presence of an interfering peak was immediately observed. The interfering cocaine oxidation peak was determined to be present between $0.88 \mathrm{~V}$ and $0.9 \mathrm{~V}$, the location of peak II for fentanyl. However, only a single peak was present with good resolution and peak shape, which could allow for the observation of peak I for fentanyl. Decent linearity was achieved for the concentration range tested with a coefficient of determination of 0.9784 (Figure 20).
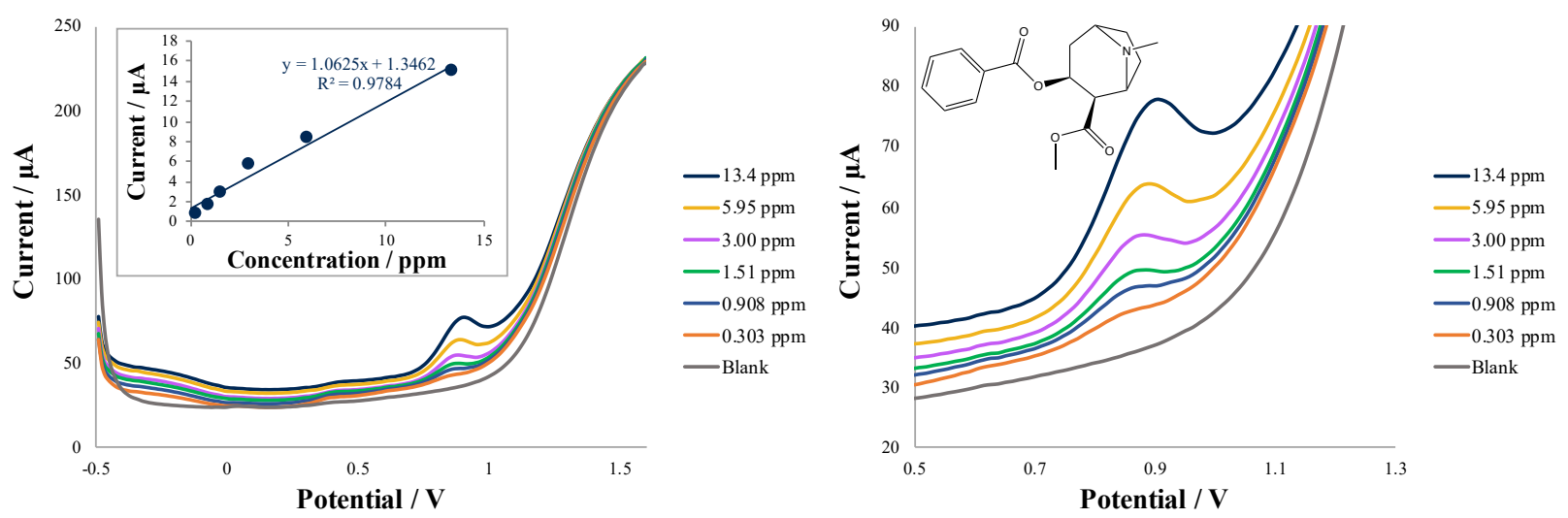

Figure 20: Adsorptive stripping square-wave voltammograms for the standard addition of cocaine in a 5 $\mathrm{mL}$ electrochemical cell containing $100 \mathrm{mM}$ Tris- $\mathrm{HCl} \mathrm{pH} 8.5$ and the corresponding standard curve.

In order to determine the effect of this cocaine peak, varying ratios of fentanyl to cocaine were analyzed. At $0.3: 10 \mu \mathrm{g} / \mathrm{mL}$, the cocaine oxidation peak overshadowed peak I for fentanyl. There was an indication of the fentanyl peak as a shoulder at $0.75 \mathrm{~V}$ (Figure 21). At a ratio of 1:10 
$\mu \mathrm{g} / \mathrm{mL}$, the presence of fentanyl peak I at $0.75 \mathrm{~V}$ became readily apparent as a shoulder (Figure 21). Finally, at a ratio of $1: 3 \mu \mathrm{g} / \mathrm{mL}$, the peak shape began to resemble that of just fentanyl with the exception of the largest peak being peak II. The fentanyl peak at $0.75 \mathrm{~V}$ became resolved from the overlapping peaks of cocaine and fentanyl peak II (Figure 21). Therefore, it was determined that peak I could be used for the identification of fentanyl when present in mixtures and that it could be possible to determine mixtures based on the ratio of peak height between peaks corresponding to the potentials of peak I and peak II for fentanyl. These results also suggest the possibility of performing a semi-quantitative analysis for the approximate concentration ratio between fentanyl and interfering compounds.
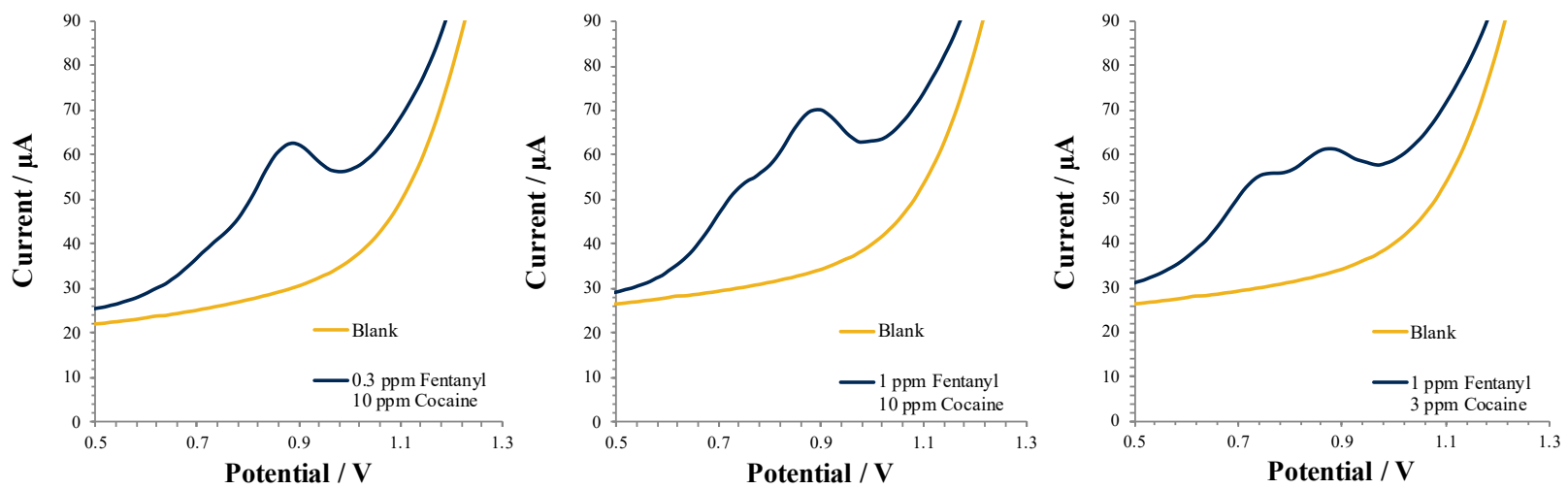

Figure 21: Adsorptive stripping square-wave voltammograms in a $5 \mathrm{~mL}$ cell with $100 \mathrm{mM}$ Tris-HCl $\mathrm{pH} 8.5$ for the analysis of fentanyl in the presence of cocaine at ratios of 0.3:10 (left), 1:10 (middle), and 1:3 (right), respectively.

Quinine was analyzed at concentrations between $0.099 \mu \mathrm{g} / \mathrm{mL}$ and $9.0 \mu \mathrm{g} / \mathrm{mL}$. Quinine was sparingly soluble in the aqueous Tris- $\mathrm{HCl}$ buffer, which was confirmed by the literature. Due to this insolubility, it could be possible that quinine may not be present as high concentrations in oral fluid or could be removed as an interfering adulterant during the analysis of powder samples when placed in the aqueous buffer. Regardless, an investigation into quinine was undertaken. After achieving solubility within the solution, increasing concentrations of quinine into the cell resulted in an oxidation peak present at $0.9 \mathrm{~V}$. Similar to cocaine; this peak would interfere with the detection of peak II for fentanyl. When compared with cocaine, the resolution of the quinine peak was better, particularly the resolution on the more positive potential side. This was an indication of higher sensitivity for quinine than cocaine, which could suggest larger interference. 
Construction of the resulting calibration curve demonstrated two regions of linearity, of which, the lower concentration region had a slope that was approximately eight times greater than the slope obtained for cocaine (Figure 22).
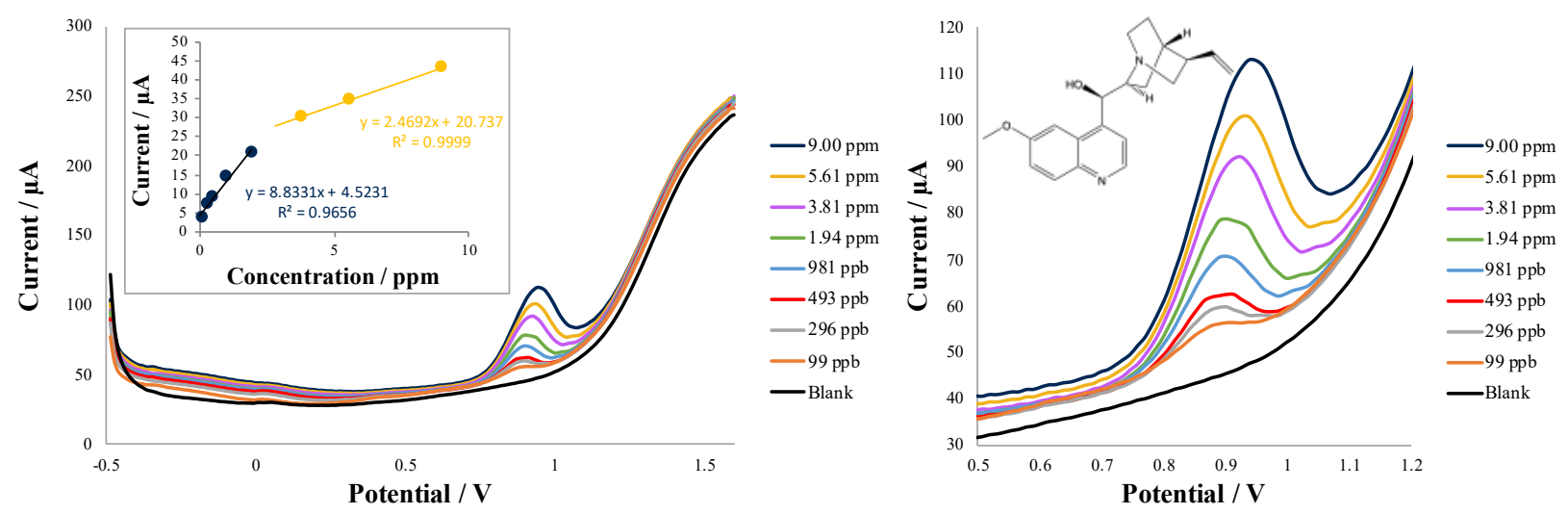

Figure 22: Adsorptive stripping square-wave voltammograms for the standard addition of quinine in a 5 $\mathrm{mL}$ electrochemical cell containing $100 \mathrm{mM}$ Tris-HCl pH 8.5 and corresponding calibration curve.

The effect of quinine on the detection capability of the method for fentanyl was tested in the same way as cocaine. The following ratios were analyzed between fentanyl and quinine: 0.3:10, $1: 10$, and $1: 3$. Unlike cocaine, no indication of fentanyl was seen at the $0.3 \mu \mathrm{g} / \mathrm{mL}$ fentanyl with $10 \mu \mathrm{g} / \mathrm{mL}$ quinine. The quinine peak was able to overshadow any electroactivity of the fentanyl within the cell. At a ratio of 1:10, peak I for fentanyl was observed as a small shoulder against the strong peak for quinine. Lastly, at a ratio of 1:3, fentanyl peak I was readily apparent at approximately $0.75 \mathrm{~V}$, resulting in the inversed peak ratio that was seen with cocaine and fentanyl (Figure 23). This ratio further provided evidence that peak I could be used for identification purposes while peaks present at the potential of fentanyl peak II could be useful indicators of the presence of interfering compounds. Similarly, should peak II be present as a smaller peak than peak I, it could indicate the presence of a pure fentanyl sample or a sample without interference and could serve as a secondary means of identification, providing stronger evidence for the presence of fentanyl in a sample. 
Part II: Electrochemical Analysis of Fentanyl
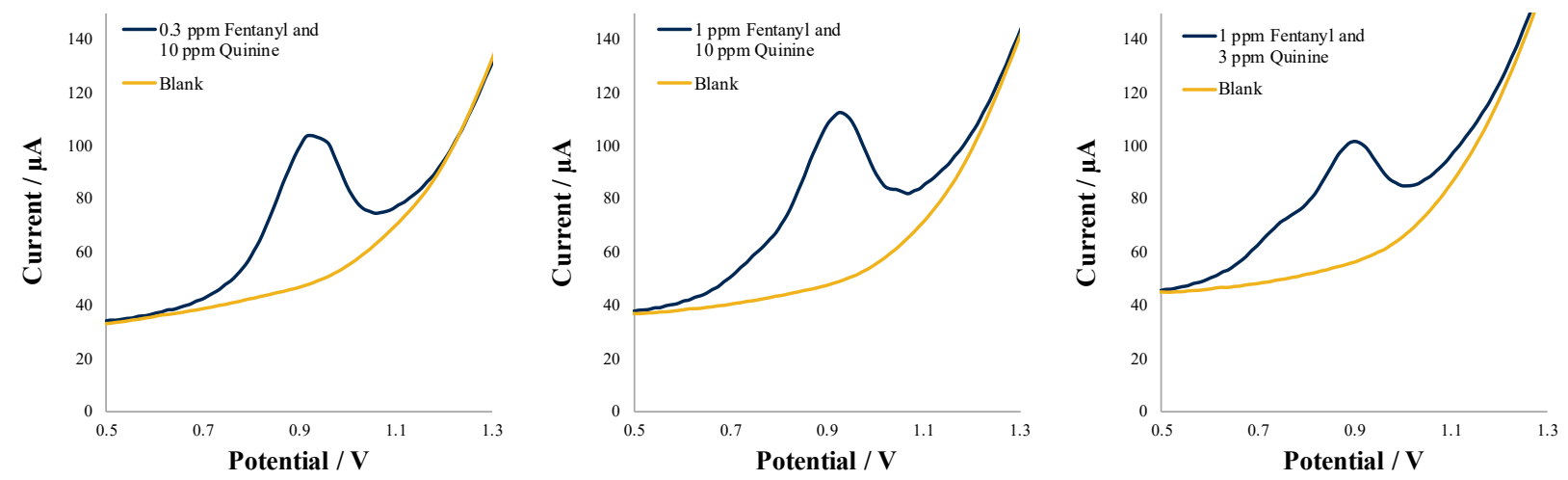

Figure 23: Adsorptive stripping square-wave voltammograms in a $5 \mathrm{~mL}$ cell with $100 \mathrm{mM}$ Tris- $\mathrm{HCl} \mathrm{pH} 8.5$ for the analysis of fentanyl in the presence of quinine at ratios of 0.3:10 (left), 1:10 (middle), and 1:3 (right), respectively.

Acetaminophen is a commonly encountered medicine for the treatment of inflammation and mild pain. Due to the general use of the compound, it is reasonable to expect that it may be naturally present in collected oral fluid samples or other biological samples. Furthermore, it is commonly used as an adulterant. Therefore, acetaminophen was analyzed between $0.151 \mu \mathrm{g} / \mathrm{mL}$ and $13.8 \mu \mathrm{g} / \mathrm{mL}$. After analysis of increasing concentrations of acetaminophen, it was observed that there was no interference at potentials corresponding to the oxidation of fentanyl. However, acetaminophen did demonstrate electroactivity through the presence of an oxidation peak generated at a potential of approximately $0.28 \mathrm{~V}$. Analysis of the calibration curve constructed demonstrated excellent sensitivity with a coefficient of determination of 0.9988 (Figure 24).
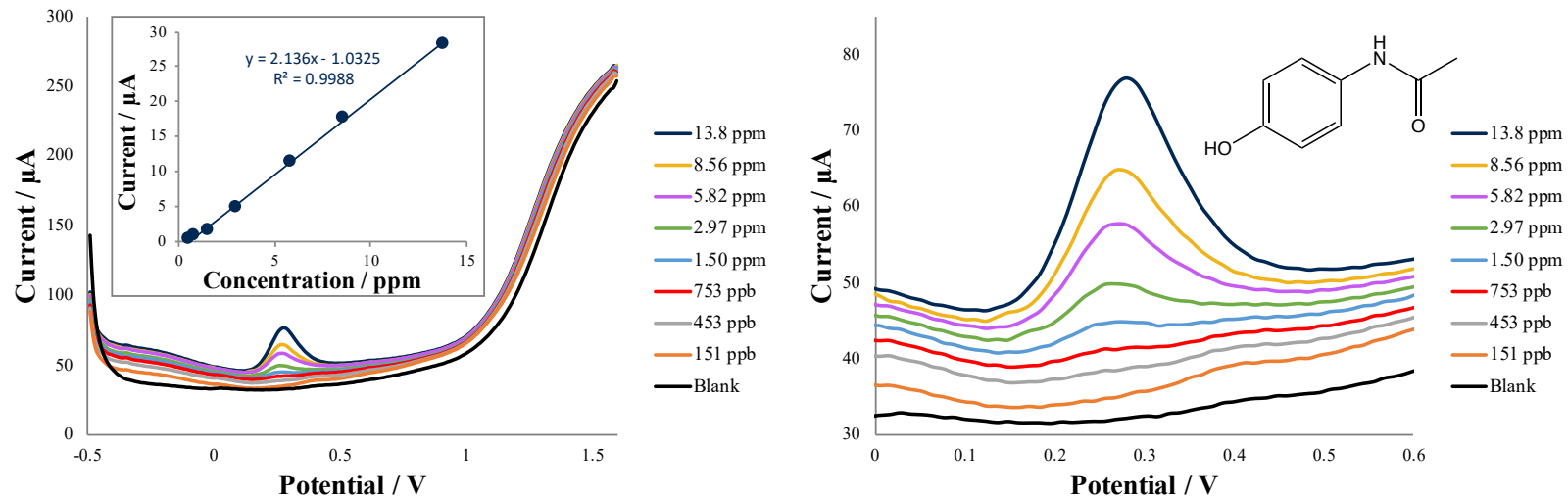

Figure 24: Adsorptive stripping square-wave voltammograms for the standard addition of acetaminophen in a $5 \mathrm{~mL}$ electrochemical cell containing $100 \mathrm{mM}$ Tris-HCl pH 8.5 and corresponding calibration curve. 
The electroactivity of acetaminophen, in this case, was considered to be fortuitous due to the potential of oxidation being centered at $0.28 \mathrm{~V}$. This potential was shown to not interfere with the signal for fentanyl while also allowing acetaminophen to be detected simultaneously (Figure 25). This behavior would prove to be a useful application when analyzing samples that may contain both compounds because, in addition to the identification of fentanyl, acetaminophen can also be identified in the sample, and possibly quantified. It was demonstrated that large concentrations of acetaminophen did not affect the ability of the method to detect both fentanyl oxidation peaks.
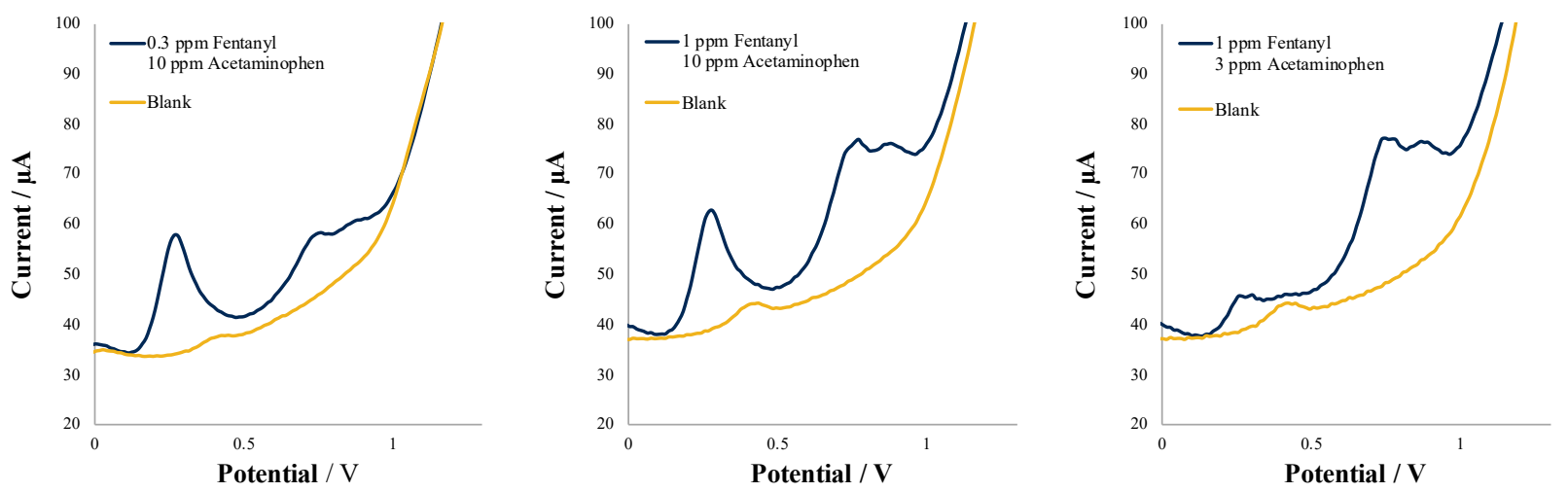

Figure 25: Adsorptive stripping square-wave voltammograms in a $5 \mathrm{~mL}$ cell with $100 \mathrm{mM}$ Tris- $\mathrm{HCl} \mathrm{pH} 8.5$ for the analysis of fentanyl in the presence of acetaminophen at ratios of 0.3:10 (left), 1:10 (middle), and 1:3 (right), respectively.

\subsection{Hypothesized Redox Mechanism}

In 2003, Garrido et al. reported the electrooxidation of heroin at a glassy carbon electrode with a glassy carbon rod auxiliary electrode and $\mathrm{Ag} / \mathrm{AgCl}$ reference electrode. The authors observed the occurrence of two peaks at basic $\mathrm{pH}$, as demonstrated in this work. ${ }^{20}$ The authors attributed these two peaks to the oxidation of the tertiary amine and the oxidation of the newly formed secondary amine. The authors reported that the peak present at less positive potential resulted from the oxidation of the tertiary amine and the peak at more positive potential was from the oxidation of the newly formed product, a secondary amine. ${ }^{20}$ Garrido et al. confirmed this through their electrochemical analysis of norheroin. This oxidation mechanism would also be supported by the direction of the applied potential, which would require the tertiary amine to be 
oxidized before the secondary amine. Furthermore, the larger peak could be expected to be attributed to the substance in higher concentration within the system.

The above-mentioned oxidation pathway has been supported in the literature. In a paper detailing the oxidation of amines, Masui et al. state the observance of two oxidation peaks for tertiary amines, a single peak for secondary amines, and the absence of oxidation peaks for primary amines. ${ }^{71}$ Furthermore, the authors provide evidence for the findings of this work and the previously mentioned work stating that amines are more easily oxidized within a basic solution, that there exists a linear relationship between the $\mathrm{pK}_{\mathrm{a}}$ of the compound and the peak potential $\left(\mathrm{E}_{\mathrm{p}}\right)$, and that oxidation of tertiary amines occurs at lower $E_{p}$ than the oxidation peak of secondary amines. ${ }^{71,72}$ The authors suggested a 2-electron oxidation process for triethylamine involving an aminium cation and reaction with water to form a secondary amine and aldehyde. ${ }^{71}$

However, these two proposed mechanisms partially disagree with Smith et al. who describe the oxidation process of substituted tertiary amines in basic solution with glassy carbon. ${ }^{73}$ Rather than describing the oxidation of a tertiary amine followed by the secondary amine, the authors proposed that both peaks were due to the same process, namely the oxidation of the tertiary amine. This phenomenon occurs due to the oxidation (at a less anodic potential) of the species adsorbed to the electrode surface and the oxidation of the analyte (at more anodic potential) due to diffusion to the electrode. ${ }^{73}$ The authors further demonstrate the analyte adsorption to the electrode through the lone pairs on the nitrogen atoms. ${ }^{73}$

Consideration of the papers mentioned above detailing possible mechanisms for the oxidation of amines, oxidation of cinnarizine by Hegde et al., ${ }^{74}$ and work demonstrating fentanyl oxidation by Goodchild et al., ${ }^{33}$ as well as this work, may aid in the elucidation of the oxidation mechanism of fentanyl. It was shown that norfentanyl did not present any oxidation peak while 4ANPP demonstrated two oxidative peaks with better peak resolution than fentanyl, confirming the location of the tertiary amine oxidation. ${ }^{33}$ We propose that peak I, seen in our work, for the oxidation of fentanyl cannot be due to oxidation of the tertiary amine present as the amide group in fentanyl as this process more likely occurs through hydrolysis in acidic conditions compared with the buffer $\mathrm{pH}$ of 8.5 used herein. Furthermore, we do not support the mechanism explained through the oxidation of the tertiary amine for peak I followed by the oxidation of the product secondary amine for peak II. Oxidation of the tertiary amine as N-dealkylation on fentanyl would result in norfentanyl. However, the suggestion of peak II arising from the oxidation of the 
secondary amine of norfentanyl is not supported in the literature, as Goodchild et al. demonstrated a complete lack of an oxidation peak. ${ }^{33}$ Therefore, both peaks must be due to the N-dealkylation of fentanyl to norfentanyl. As this work demonstrated, peak current increased as the amount of time stirred increased, suggesting that an adsorption process was occurring within the system. In addition, with much longer times, the difference in peak current decreased, further suggesting an adsorption process through the observable saturation of the electrode surface based on peak current versus time stirred. As such, it is proposed that the oxidative mechanism of fentanyl, resulting in peak I and peak II in this work, is due only to the oxidation of the tertiary amine through Ndealkylation. The presence of the two peaks can be explained through the oxidation processes of adsorbed species and species diffusing to the electrode surface. Cyclic voltammetry was utilized as a means to further support this claim. CV of fentanyl was analyzed under varying scan rates between $0.1 \mathrm{~V} / \mathrm{s}$ and $0.75 \mathrm{~V} / \mathrm{s}$. Plots of peak current versus the scan rate (adsorption) and the square root of the scan rate (diffusion) were analyzed. Linearity was observed in both plots suggesting that both processes were occurring (Figure 26).
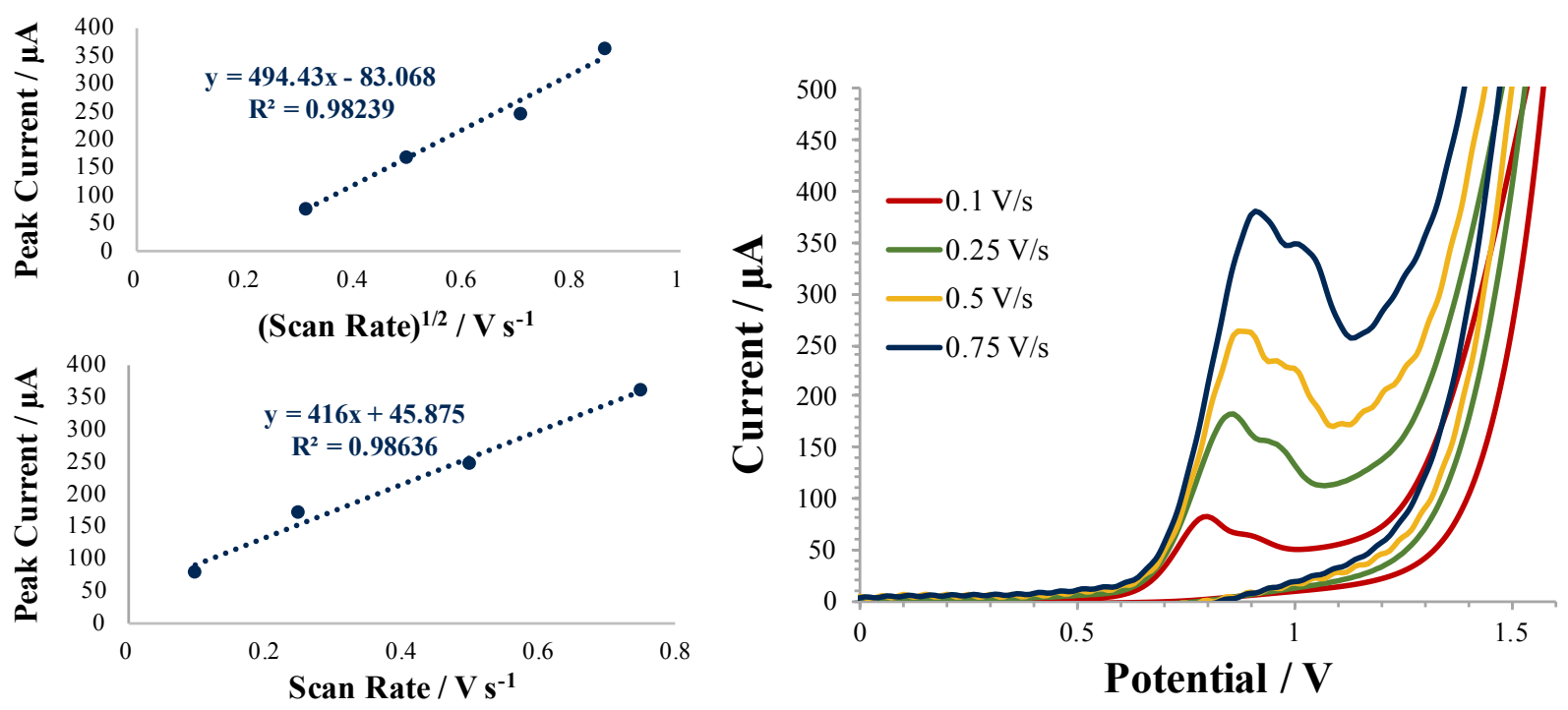

Figure 26: Cyclic voltammograms collected at varying scan rates (right) to demonstrate that the electrooxidation of fentanyl involves both diffusion (top left) and adsorption (bottom left).

Further support of both an adsorption and diffusion process is the presence of an oxidation peak in the analysis of 4-ANPP that demonstrates improved peak resolution for peak I and peak II. ${ }^{33}$ We suspect that this is due to a higher affinity of 4-ANPP for the electrode surface due to decreased steric hindrance and greater access to the lone pair as a result of the absence of the amide 
group. Lastly, this process was determined to be an irreversible electron transfer for fentanyl, which was supported through analysis of fentanyl in a drop by successive scans via cyclic voltammetry showing the decrease in peak current and shape with each successive scan (Figure 27). This experiment demonstrated the irreversible nature of the oxidation due to the lack of a reduction peak and the decreasing oxidation current, suggesting that the species is being used up rather than recycled in the process. The proposed electrooxidation mechanism of fentanyl can be seen in Figure 28 as well as the previously proposed mechanism.

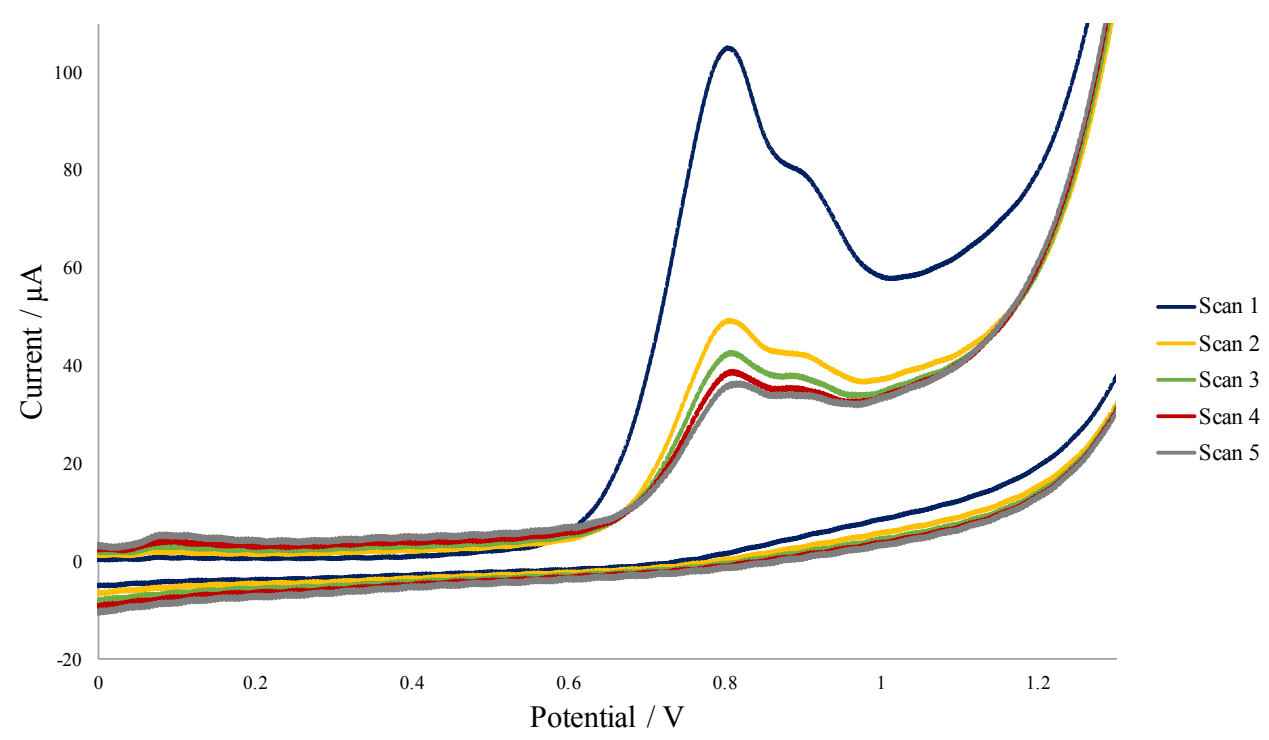

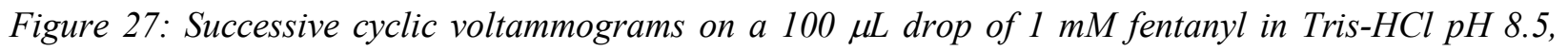
demonstrating the irreversible electron transfer process. 
Chapter 3: Results and Discussion

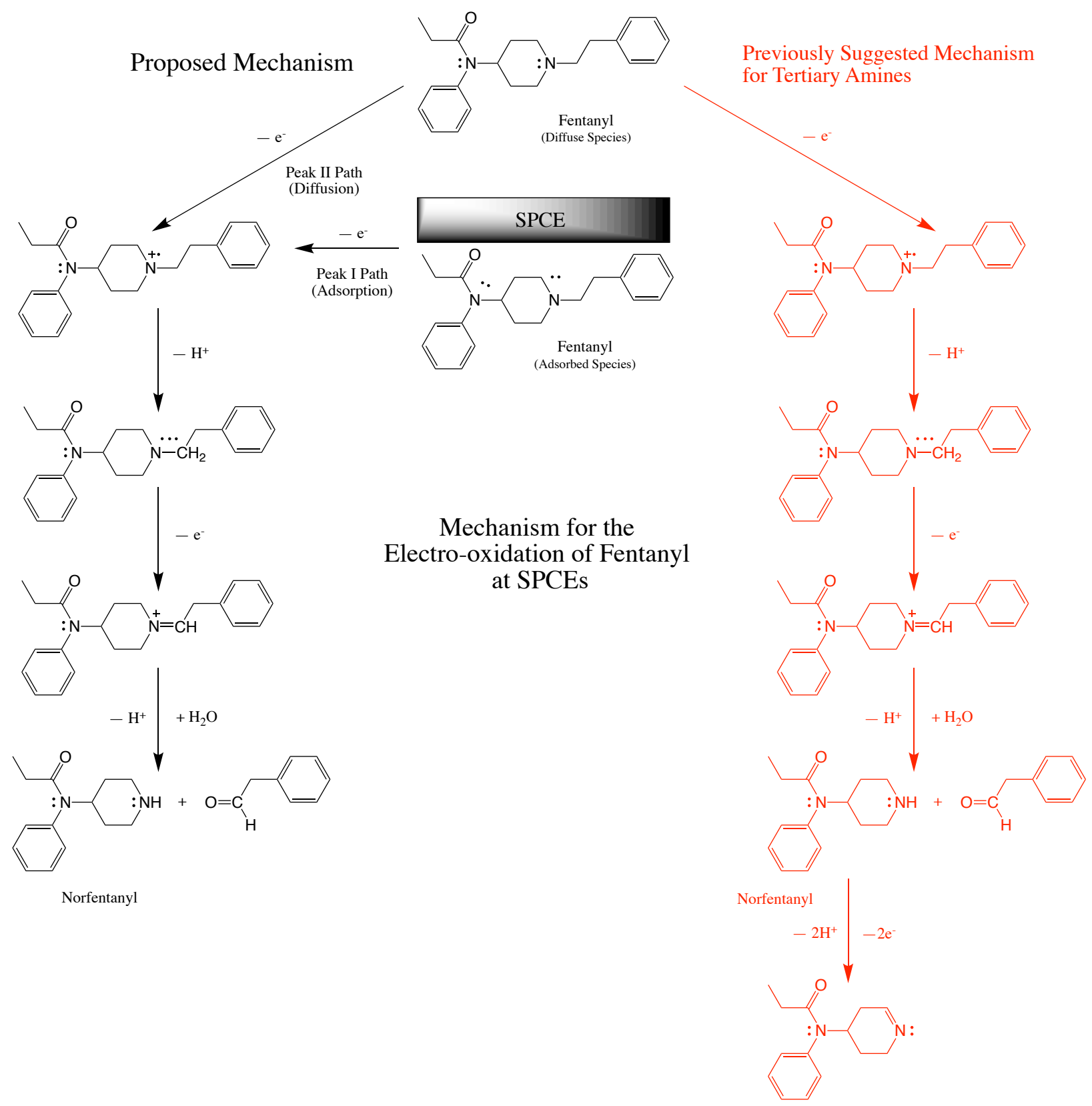

Figure 28: Proposed mechanism for the electro-oxidation of fentanyl and the previously suggested mechanism for tertiary amines. 
In order to provide further evidence about the electro-oxidation process occurring, samples of fentanyl analyzed as a drop on the surface were tested using the AdS-SWV procedure. However, drops were analyzed over a varying number of AdS-SWV scans $(0,10,20,40$, and 80 scans). These drops were then transferred to vials and analyzed using the developed LC/MS/MS method detailed in Part III of this thesis. Analysis of these samples demonstrated the presence of fentanyl and some 4-ANPP, but more importantly, the lack of norfentanyl in the 0-scan (fentanyl stock) sample. Upon an increasing number of scans, the amount of norfentanyl can be seen to increase, demonstrating the electro-oxidation of fentanyl to norfentanyl (Figure 29).

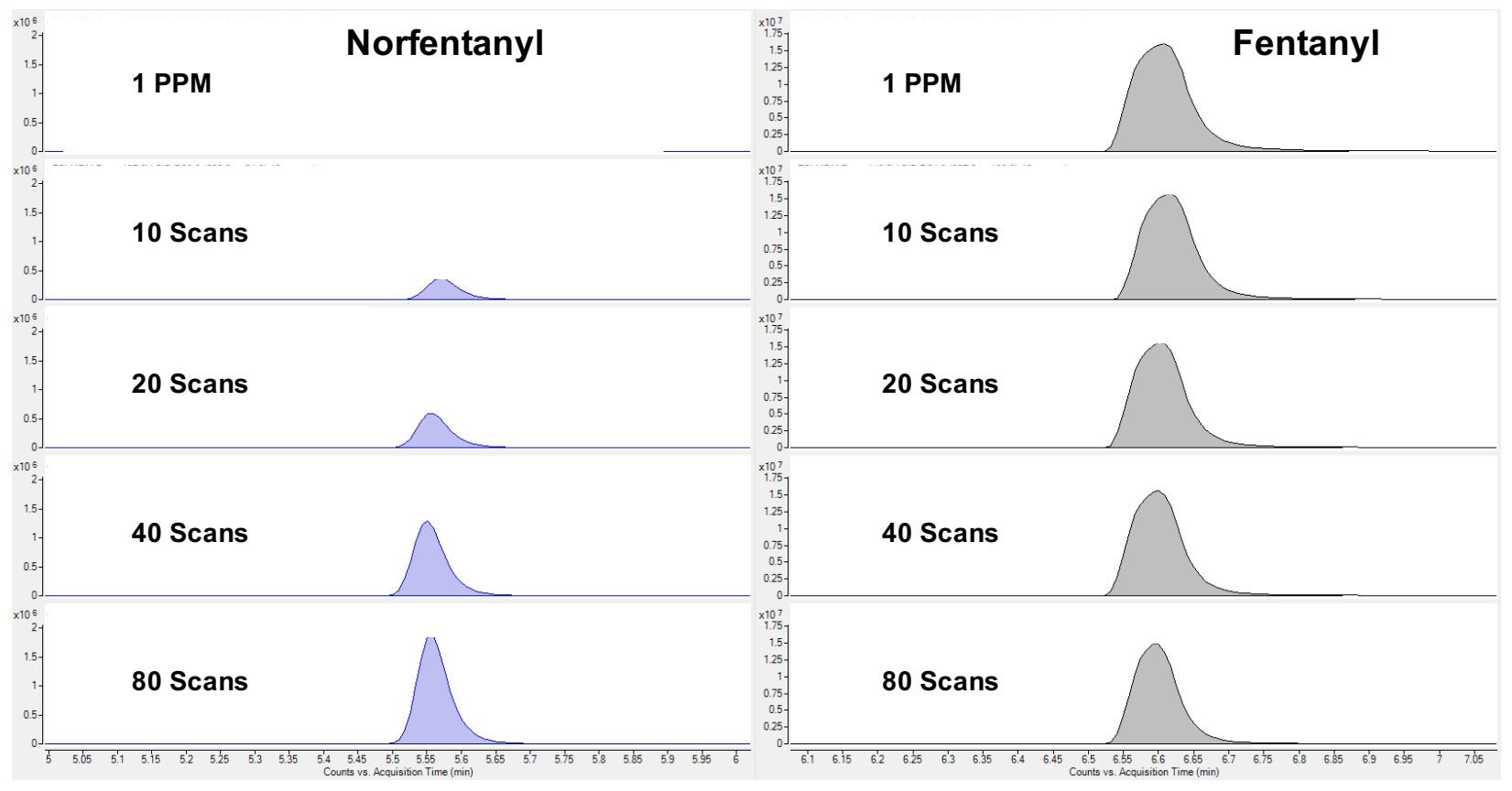

Figure 29: LC/MS/MS results following the electrochemical analysis of fentanyl demonstrating the increasing concentration of norfentanyl, supporting the proposed oxidation pathway.

\subsection{Nanomaterial Modifications}

Various types of nanomodifications were explored using screen-printed carbon electrodes to determine whether another type of surface could improve the sensitivity or linearity of the detection of fentanyl.

Rhodium nanoparticles were deposited on the working electrode surface $\left(\mathrm{SPC}_{\mathrm{Rhnps}} \mathrm{E}\right)$ through the application of $-0.25 \mathrm{~V}$ for 480 seconds. When utilizing the adsorptive stripping approach for 320 seconds with the optimized square-wave procedure, calibration curves were constructed between $0.076 \mu \mathrm{g} / \mathrm{mL}$ and $6.88 \mu \mathrm{g} / \mathrm{mL}$ of fentanyl. Pre-treatment of the working 
electrode surface was performed before deposition of the Rhodium onto the surface. Rhodium experiments demonstrated promise in their ability to detect fentanyl and exhibited two regions of linearity. An example calibration curve and voltammograms are shown in Figure 30. Only a single peak, corresponding to the potential of peak I, was observed for the majority of the concentrations. At higher concentrations, the second peak became apparent, but only peak I was assessed in the calibration curve.
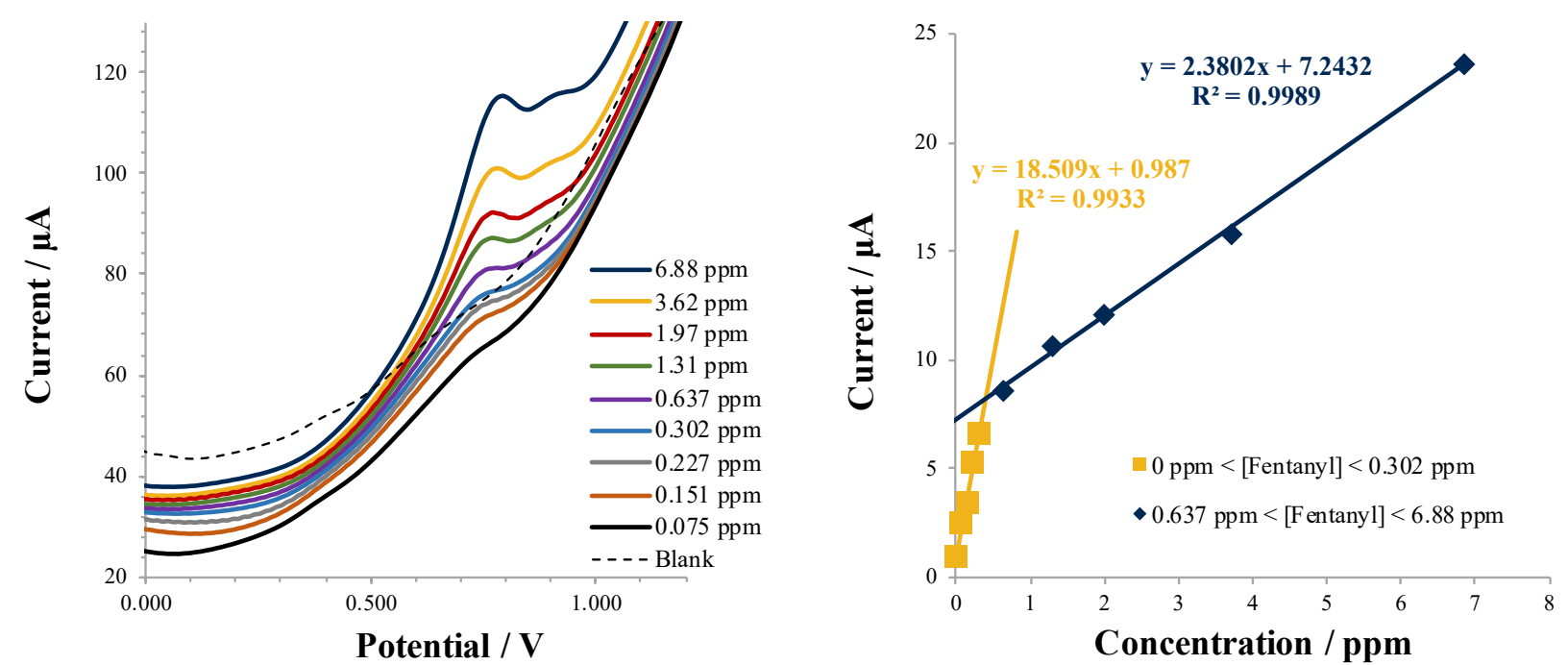

Figure 30: AdS-SWV for increasing concentrations of fentanyl in Tris-HCl at a modified $S P C_{R h n p s}$ (left) and constructed calibration curve for fentanyl (right).

Multiwalled carbon nanotubes were explored as modifications to the working electrode surface ( $\left.\mathrm{SPC}_{\mathrm{MWCNT}} \mathrm{E}\right)$. Surface exploration occurred before the use of the 320 second equilibration time. Therefore, these experiments were carried out using the optimized SWV technique along with a 20 second equilibration time. Linearity was achieved using MWCNTs; however, only a single peak was able to be seen in the potential range corresponding to fentanyl (Figure 31). Furthermore, the method was unable to detect the lowest concentration tested, which was 336 $\mathrm{ng} / \mathrm{mL}$. Three replicate curves were analyzed. 
Part II: Electrochemical Analysis of Fentanyl
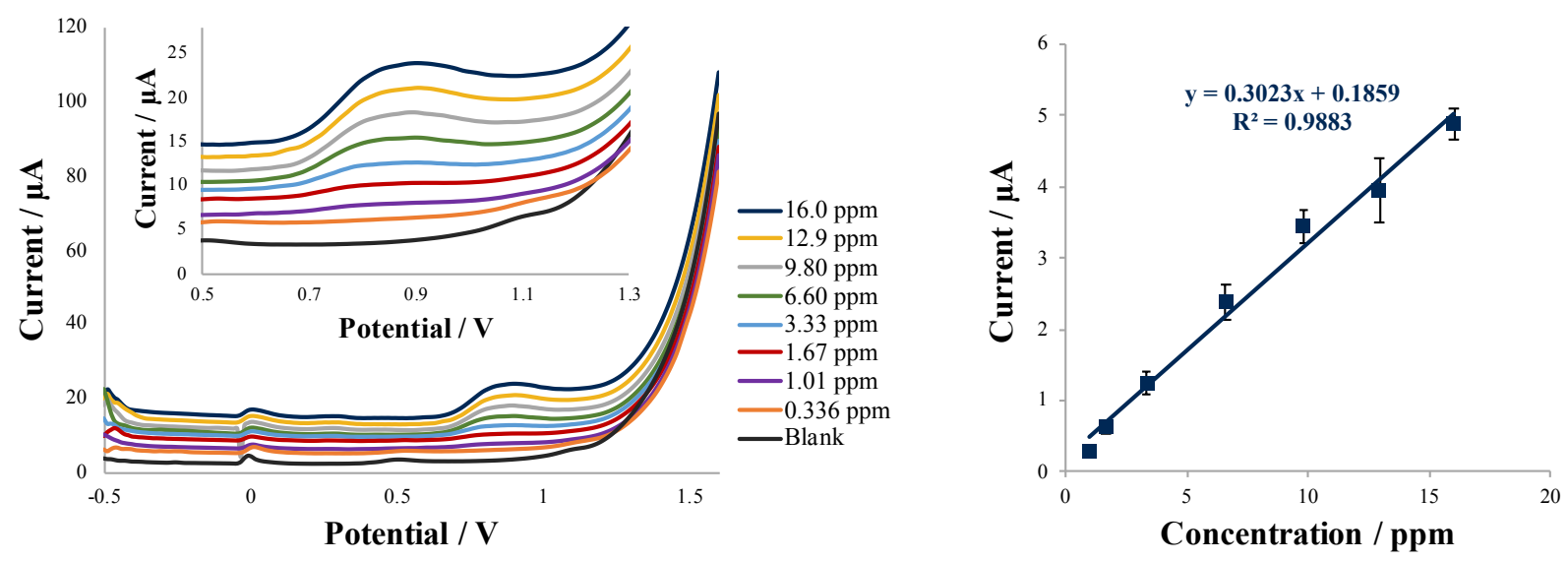

Figure 31: SWV for increasing concentrations of fentanyl in Tris-HCl at a modified SPC $C_{M C N T E}$ (left) and constructed calibration curve for fentanyl (right).

The use of graphene oxide was investigated next. Following the reduction of the graphene oxide, the surface was analyzed similarly to the MWCNTs. Two replicates using $\mathrm{SPC}_{\mathrm{rGOEs}}$ were assessed. Extremely poor linearity was observed for this approach and demonstrated a ' $U$ ' shape in the constructed calibration curve from two replicate experiments. The resulting voltammograms and calibration curve can be seen in Figure 32. A high background current could be seen that diminished with each new concentration, owing to the seemingly flipped voltammograms with the highest concentration below lower concentrations. At higher concentrations of fentanyl, the fentanyl peaks could be distinguished from the background and both peak I and peak II were observable. 

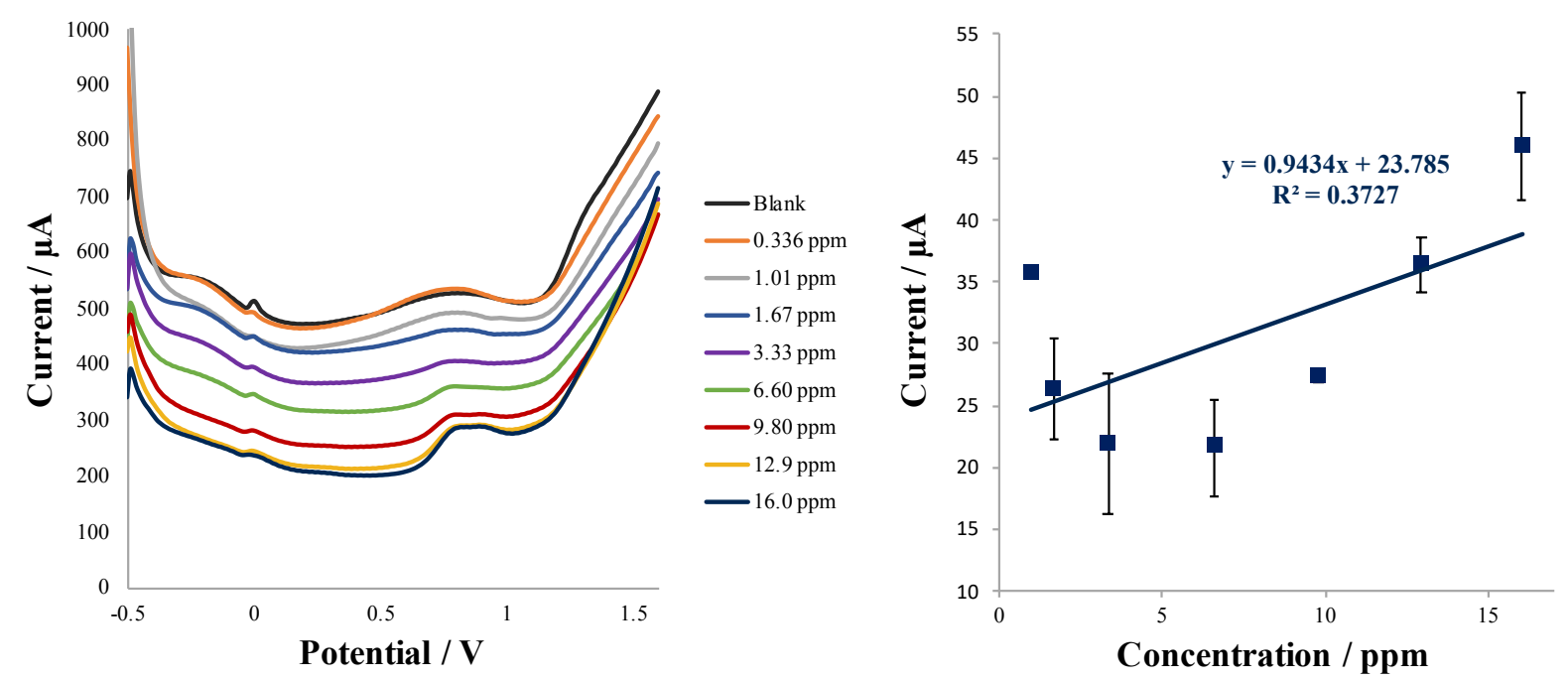

Figure 32: SWV for increasing concentrations of fentanyl in Tris-HCl at a modified $S P C_{r G O E}$ (left) and constructed calibration curve for fentanyl (right).

Gold and silver nanoparticles were explored due to their simplicity of formation on the electrode surface and inherent ability to boost the signal. Silver solutions of $0.1 \mathrm{mM}$ and $5 \mathrm{mM}$ were investigated with a deposition time of 10 seconds. Similar linearity was achieved for both conditions; however, the replicate performed from the deposition of the $0.1 \mathrm{mM}$ silver solution demonstrated higher slopes in the calibration curve, suggesting greater sensitivity. Figure 33 shows the resulting adsorptive stripping square-wave voltammograms for analysis of fentanyl. Both peak I and peak II can be seen at their corresponding potentials, as well as a large peak around $0 \mathrm{~V}$. This peak was seen to diminish with each successive concentration run and can be attributed to the interaction of silver and chloride within the system.

Gold nanoparticles also allowed for the detection of fentanyl. However, only a single peak was present rather than both peaks. Also, a large oxidation peak was observed at approximately 1 $\mathrm{V}$. This can be attributed to the oxidation related to the presence of the gold nanoparticles on the surface of the electrode. This peak, due to the presence of gold, was seen in previous experiments involving the use of gold nanoparticles. It was determined that the use of gold nanoparticles might not provide an acceptable detection platform for fentanyl due to the fentanyl peaks appearing beside the large gold peak. The fentanyl peak was also observed to be broad with this modification approach. 

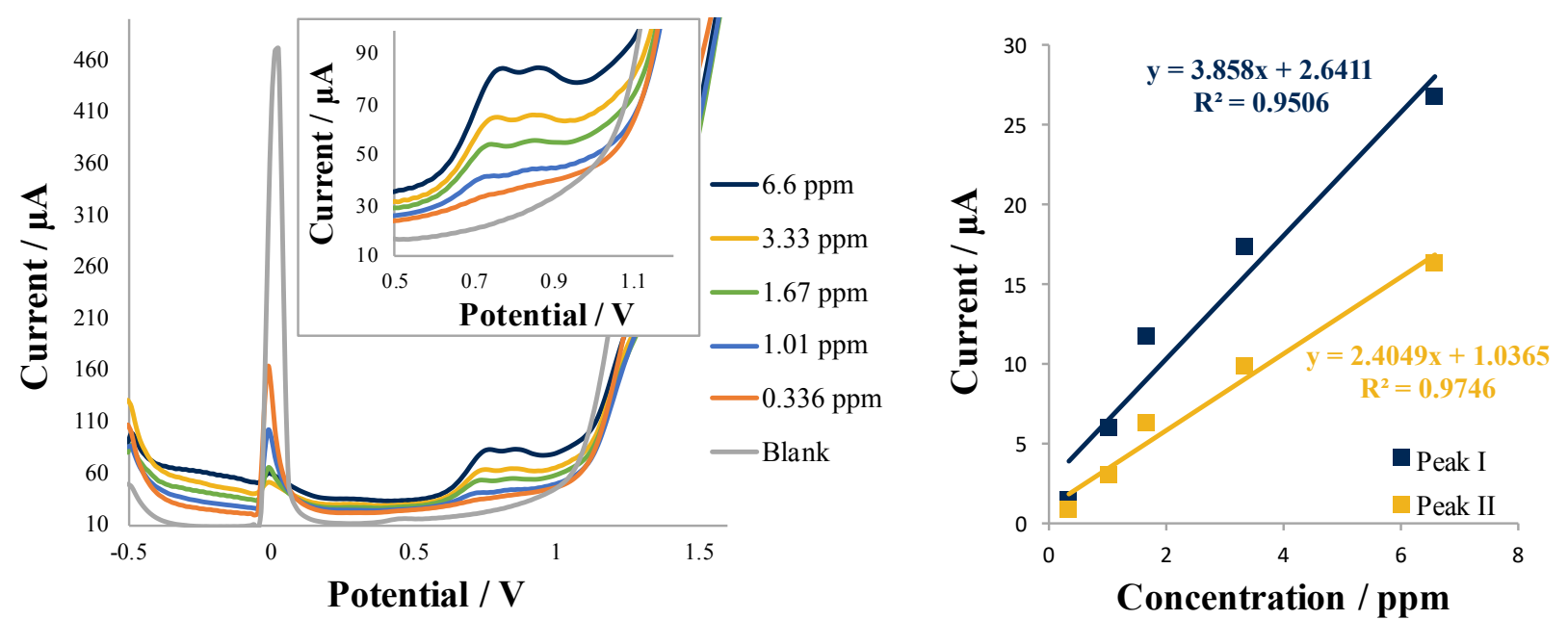

Figure 33: SWV for increasing concentrations of fentanyl in Tris-HCl at a modified $S P C_{\text {Agnps }} E$ (left) and constructed calibration curve for fentanyl (right).

The combination of silver nanoparticles with MWCNTs was explored. For each electrode, a silver solution of $0.1 \mathrm{mM}$ was used for the deposition of the nanoparticles for 10 seconds. MWCNT solutions were made to $0.015,0.075,0.15,0.3,3 \mathrm{mg} / \mathrm{mL}$ and added to the electrode surface. The electrode response was assessed based on peak current height and on peak shape for both peak I and peak II for fentanyl. The $\mathrm{Ag}_{\mathrm{nps}} / \mathrm{MWCNT}(3 \mathrm{mg} / \mathrm{mL})$ electrode produced the highest current response for the concentrations tested. However, peak II was lost in the lowest concentration, and a noisier signal was observed. The second-best modification was with the MWCNT concentration of $0.075 \mathrm{mg} / \mathrm{mL}$. Both peaks were seen with higher peak currents than the other electrodes. Peak shape was also improved from the remaining electrodes. This approach could prove a useful detection strategy; however, sensitivity would need to be improved since the lowest concentration tested was $671 \mathrm{ng} / \mathrm{mL}$. Figure 34 demonstrates the voltammograms and calibration curve obtained for the electrode modified with $\mathrm{Ag}_{\mathrm{nps}}$ and $0.075 \mathrm{mg} / \mathrm{mL} \mathrm{MWCNTs}$. The linearity achieved using this modification technique was not as desirable; however, other concentrations and replicates would be needed to assess the variability and to determine if two regions of linearity are present. 

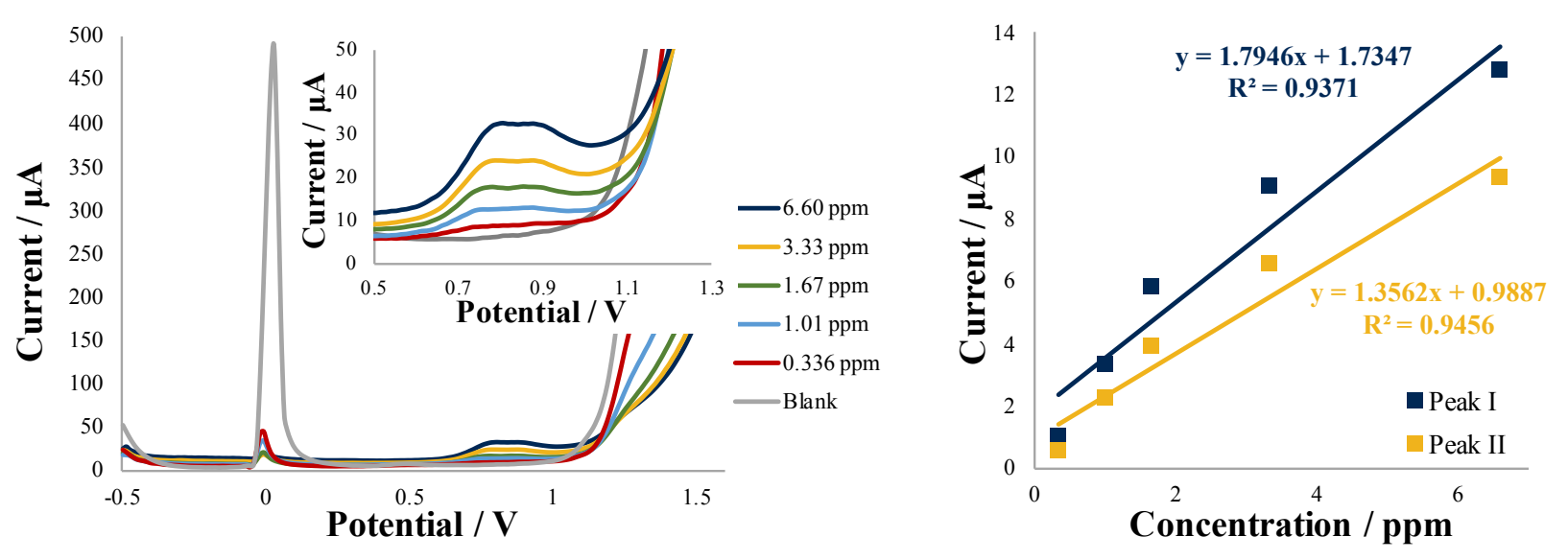

Figure 34: SWV for increasing concentrations of fentanyl in Tris-HCl at a modified $S P C_{\text {Agnps/MWCNTE (left) }}$ and constructed calibration curve for fentanyl (right).

Other possible modifications and detection methods were attempted including the use of SPCEs with carbon paths, unmodified and with silver nanoparticles; a glassy carbon conventional electrode; and a conventional silver electrode. These methods demonstrated poor linearity and were not investigated further. However, the use of the conventional silver and glassy carbon electrodes could represent a possibility for testing, as lower concentrations were not examined to determine two regions of linearity. Nonetheless, the use of conventional electrodes would pose a problem for work performed in the field. A comparison of the tested modifications, including the final method on bare carbon can be found in Table 5 .

\subsection{Anodic Stripping Square-Wave Voltammetry}

Rather than utilizing the anodic pre-treatment and 320 second equilibration time, the use of an anodic stripping method was investigated for use prior to the application of the square-wave method for the detection of fentanyl. This approach was explored due to the proposed adsorption mechanism, which could improve detection capabilities through amperometric adsorption of fentanyl before oxidation. Anodic stripping conditions were assessed previously, and a potential of $0.1 \mathrm{~V}$ was determined to be optimal based on peak potential, slope, and correlation coefficient. No significant difference was seen between 60 seconds versus 120 seconds versus 520 seconds. Anodic stripping at $0.1 \mathrm{~V}$ for 120 seconds was considered to be the best parameter due to the shape 
Part II: Electrochemical Analysis of Fentanyl

of the peaks. However, the performance was comparable to the pre-treatment method and was not further explored.

Table 5: Comparison between electrode modifications/detection methods

\begin{tabular}{|c|c|c|c|c|c|}
\hline Modification & Technique & $\begin{array}{l}\text { Lowest Concentration } \\
\text { Detected }(\mu \mathrm{g} / \mathrm{mL})\end{array}$ & $\begin{array}{l}\text { Linear Range } \\
(\mu \mathrm{g} / \mathrm{mL})\end{array}$ & $\mathrm{R}^{2}$ & $\begin{array}{l}\text { Current in } 1 \mu \mathrm{g} / \mathrm{mL} \\
\text { Concentration }(\mu \mathrm{A})\end{array}$ \\
\hline Pre-treatment & $\begin{array}{l}\text { AdS- } \\
\text { SWV }\end{array}$ & 0.076 & $\begin{array}{c}0.075 \text { to } 0.64 \\
1.3 \text { to } 6.9\end{array}$ & $\begin{array}{l}0.9948 \\
0.9909\end{array}$ & $\begin{array}{l}4.9(0.64 \mu \mathrm{g} / \mathrm{mL}) \\
8.54(1.3 \mu \mathrm{g} / \mathrm{mL})\end{array}$ \\
\hline Rh nps & $\begin{array}{l}\text { AdS- } \\
\text { SWV }\end{array}$ & 0.076 & $\begin{array}{c}0.075 \text { to } 0.64 \\
1.3 \text { to } 6.6\end{array}$ & $\begin{array}{l}0.9933 \\
0.9989\end{array}$ & $8.62(0.64 \mu \mathrm{g} / \mathrm{mL})$ \\
\hline MWCNTs & SWV & 1.01 & 1.01 to 16 & 0.9883 & 0.290 \\
\hline rGO & SWV & 0.336 & NA & $\begin{array}{l}\text { Not } \\
\text { Linear }\end{array}$ & -- \\
\hline Ag nps & SWV & 0.336 & 0.336 to 6.6 & 0.9506 & 5.90 \\
\hline Ag/MWCNTs & SWV & 0.336 & 0.336 to 6.6 & 0.9370 & 3.33 \\
\hline Au nps & SWV & 0.336 & 0.336 to 6.6 & 0.9977 & 1.96 \\
\hline
\end{tabular}




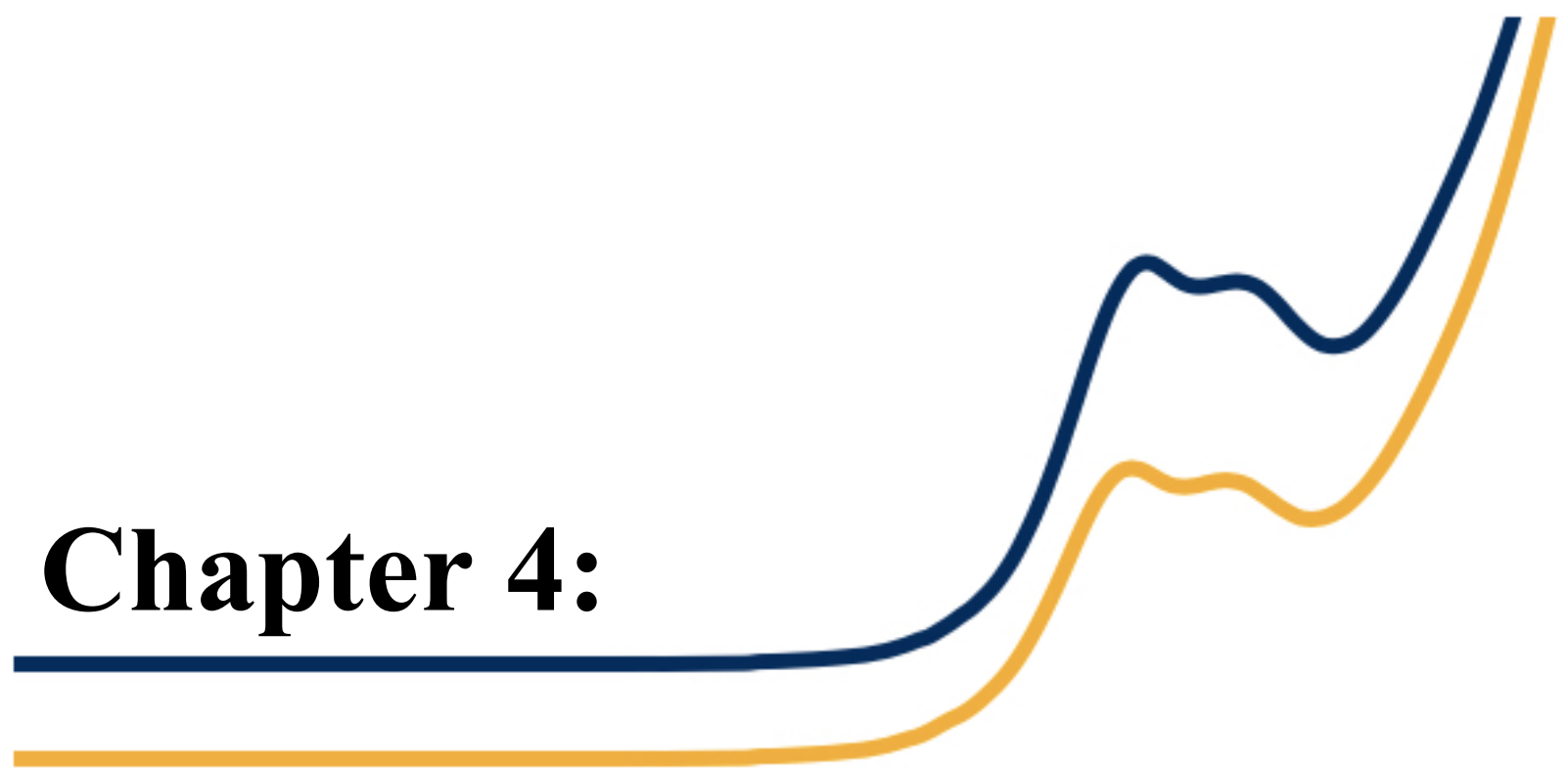

Conclusions and Future Work 


\subsection{Conclusions}

The electroactivity of fentanyl at a screen-printed carbon electrode was demonstrated through the use of adsorptive stripping square-wave voltammetry. The electro-oxidation was observed to take place through two separate electron transfer events, giving rise to two oxidation peaks. The first peak, referred to as peak I, was present at a potential of approximately $0.75 \mathrm{~V}$. The second peak, referred to as peak II, was present at approximately $0.88 \mathrm{~V}$. The oxidative behavior of fentanyl was attributed to the presence of the amine groups within the molecule. The electrooxidative mechanism for fentanyl is hypothesized to be a result of oxidation of the tertiary amine present in the piperidine ring, resulting in dealkylation to form norfentanyl.

The presence of peak I was attributed to the oxidation of adsorbed fentanyl species, while peak II was attributed to the oxidation of diffuse fentanyl species. The demonstrated detection method was shown to provide good reproducibility and limits of detection for analysis within an electrochemical cell and analysis within a drop on the electrode surface. The limit of detection for analysis within a cell was calculated to be approximately $145 \mathrm{ng} / \mathrm{mL}$ fentanyl, and the limit of detection within a drop was calculated to be about $530 \mathrm{ng} / \mathrm{mL}$. However, fentanyl was able to be detected below these calculated values, as evidenced by the presence of oxidation peaks as low as $76 \mathrm{ng} / \mathrm{mL}$ for cell and approximately $300 \mathrm{ng} / \mathrm{mL}$ for drop analysis. A peak could be observed for analysis in a cell at $46 \mathrm{ng} / \mathrm{mL}$ fentanyl. The reproducibility of the method, expressed as RSD, in the cell, and in the drop, was $12 \%$ and $13 \%$ for peak I and $18 \%$ and $15 \%$ for peak II, respectively. The use of unmodified SPCEs allowed this detection method to be simple, cheap, reproducible, and did not suffer from electrode degradation due to storage time or conditions. Although the electrode surface was unmodified, it was shown that there was minimal interference from commonly encountered drugs and adulterants found with fentanyl. Methamphetamine, caffeine, and acetaminophen did not interfere with the detection of fentanyl, allowing peak I and peak II to be observed at various ratios, providing identification. Analysis of cocaine and quinine resulted in oxidation peaks overlapping with peak II of fentanyl. At dilute concentration ratios, the oxidation of fentanyl was not able to be detected when present with quinine and, to a lesser extent, cocaine. However, with less dilute concentration ratios, peak I of fentanyl was observed, allowing for the identification of fentanyl within the sample and generation of the identification criteria, the presence of a peak at approximately $0.75 \mathrm{~V}$. The presence of the second peak along with the ratio 
between peak I and peak II may allow for further information to be discovered about the sample such as the presence of a mixture versus a pure sample.

The oxidative mechanism was proposed based on the literature available for the oxidation of amines, as well as, voltammetric data present for fentanyl and related compounds. The proposed mechanism rejects some previously hypothesized oxidation mechanisms of tertiary amines where the presence of two peaks was observed. Where others have suggested the two-step oxidation process of the tertiary amine followed by the oxidation of the newly formed secondary amine product, we agree with literature supporting the effect of adsorption of the tertiary diamine to the electrode surface and present the mechanism whereby the observed peaks are a result of oxidation of species diffusing to the surface and of species adsorbed to the surface, shifting the potential.

Several other detection surfaces were investigated and were shown to have promise in future work. The use of rhodium, gold, and silver nanoparticles, as well as MWCNTs, was demonstrated to achieve response to fentanyl with acceptable linearity. However, the gold and silver surfaces and MWCNTs did not demonstrate the same sensitivity as seen with rhodium or the AdS-SWV SPCE method, although the fully optimized method was not explored.

The combined use of anodic stripping with SWV was investigated and shown to demonstrate good linearity and peak shape, however, there was not a noticeable advantage to using this process over the pre-treatment process, as evidenced by the calibration curves obtained.

\subsection{Future Work}

Future work related to this project will be focused on the improvements of the electrochemical sensor for fentanyl. Modifications to the electrode surface will be explored in more depth in order to extend the limits of detection lower and achieve better reproducibility. To this end, the electrochemical technique will be further investigated and optimized to determine a method that is suitable for the analysis of multiple analytes in such a way that selectivity can be achieved with low detection limits. More work will be conducted to improve the quasi-quantitative nature of the identification of the target analytes to provide better quantitative data. Fentanyl analogs may be explored to determine the selectivity of the technique and other techniques for differentiating between the fentanyl analogs. Furthermore, the electro-oxidative mechanism of fentanyl may be investigated through the analysis of similar compounds, metabolites, degradation 
products, and precursors to elucidate the oxidative mechanism further. The analysis of these compounds will provide information regarding the oxidative behavior resulting in peak I and peak II seen in this work. Investigations into the reasons behind the two regions of linearity will also be undertaken to explain this observed phenomenon.

Expansion of the capabilities of simultaneous multi-analyte analysis will be explored to provide a means of presumptive identification of unknown substances in the field. Future work may also focus on the oral fluid matrix. To this end, methods will be developed to allow for the electrochemical detection of target analytes in oral fluid both in the laboratory and in the field. Field analysis will require minimal sample preparation and ease of testing. These investigations will be targeted toward improving the capabilities of electrochemical detection methodologies for use in forensic science to provide more data toward the future incorporation of electrochemistry into the SWGDRUG categories. 
Part III: LC/MS/MS Analysis of Fentanyl and its Analogs in Oral Fluid

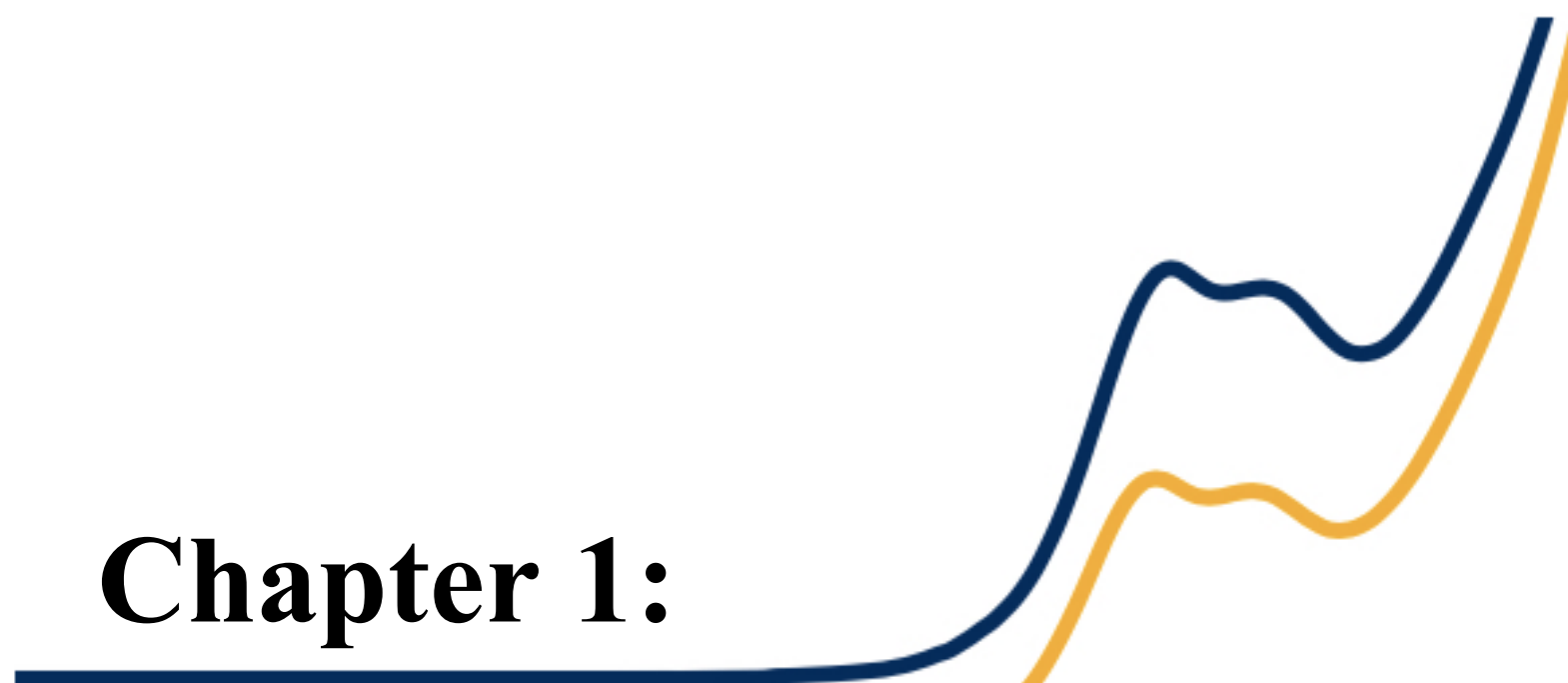

Relevant Literature Review 


\subsection{Oral Fluid}

The combined secretions from the serous-cells and mucous-cells of the parotid, sublingual, and submandibular salivary glands in the human mouth contribute to the make-up of oral fluid. ${ }^{75}$ These glands secrete approximately $25 \%, 4 \%$, and $71 \%$ of the liquid termed oral fluid, respectively. ${ }^{40,75}$ Between $800-1000$ other minor glands contribute to this mixture. The composition of the oral fluid is: $98 \%$ water, $0.7 \%$ proteins (amylases), and $0.26 \%$ glycoproteins (mucins) and electrolytes. These values are attributed to the different types of cells, and generally, results in a slightly acidic environment, although the $\mathrm{pH}$ of oral fluid can range between 5.5 to 7.9. ${ }^{75}$ The serous cells produce an aqueous solution that is high in electrolytes and is colorless, while the mucosal contribution contains a viscous mixture of proteins, polysaccharides, and glycoproteins. Approximately $500-1500 \mathrm{~mL}$ of oral fluid is produced at an average rate of 0.6 $\mathrm{mL} / \mathrm{min}$ for a single day. ${ }^{75}$

The use of oral fluid as a matrix for analysis dates back to the mid-19 $9^{\text {th }}$ century and early 1900 s but was first proposed for detecting drugs in forensic settings in the 1970 s. $^{75}$ This has progressed to be targeted toward driving under the influence (DUI) cases for on-site testing since oral fluid collection is considered non-invasive. ${ }^{40,75}$

The "metabolic profile" of drugs in oral fluid differs from that of blood and urine. The detection window in this matrix is several hours up to two days or more for some analytes. ${ }^{75}$ Oral fluid has the advantage of being able to collect a sample that is non-invasive without the problems associated with gender concerns, accessibility, and adulteration. ${ }^{40,75}$ Furthermore, the presence of drugs within blood and plasma should be accessible within the saliva since oral fluid can be thought of as a filtrate of blood and is derived from plasma. ${ }^{40,75}$ The major advantage of an oral fluid matrix is that the parent drugs are often present in higher concentration versus their metabolites when compared to blood. However, comparisons between oral fluid concentrations and the levels of the drug in the blood are rarely reliable. ${ }^{75}$ Finally, the low protein content means that there should be less binding of drugs than in plasma. ${ }^{40}$

Multiple challenges are also encountered with an oral fluid matrix. One of those disadvantages is the detection window for drugs, but this is also an advantage because oral fluid can then be used to assess recent drug use. ${ }^{75}$ Other disadvantages are changes in $\mathrm{pH}(\mathrm{pH} 7.4$ for 
stimulated saliva), ${ }^{40}$ low analyte levels, and contamination from drugs administered orally or through intranasal administration. ${ }^{75}$

Drugs enter the oral fluid through three mechanisms: passive diffusion, ultrafiltration, and active secretion from blood. These mechanisms are affected by the molecular weight of the compound, degree of ionization in oral fluid $\mathrm{pH}$, and lipid solubility. ${ }^{75}$ Ionized, protein-bound, and molecules greater than $500 \mathrm{Da}$ are generally hindered. Typically, basic and ionized drugs are more easily partitioned from the blood to the oral fluid and have oral fluid-blood partition (OF/P) ratios greater than $1 .{ }^{75}$ Common opioids such as codeine, methadone, and morphine have OF/Ps of 8.8, 2.9, and 9.8, respectively. This suggests that the concentrations of these drugs can be found higher in oral fluid than in blood. Additionally, an approximate 100-fold increase in the partition ratio can be seen when the drug is snorted or smoked. ${ }^{75}$ Being a basic compound with a $\mathrm{pK}_{\mathrm{a}}$ of 8.4 , fentanyl will be expected to have an OF/P ratio greater than 1 since it will remain in the ionized state at physiological $\mathrm{pH}$ and may be transported into the oral fluid with the aid of $p$-glycoprotein. ${ }^{76}$ In chronic pain patients, the average oral fluid concentration was found to be $4.5 \pm 2.6 \mathrm{ng} / \mathrm{mL}$ for fentanyl and $1.5 \pm 0.8 \mathrm{ng} / \mathrm{mL}$ for norfentanyl. ${ }^{77}$ Another study from 11 cancer patients with a transdermal patch had an average oral fluid concentration of $3.334 \mathrm{ng} / \mathrm{mL}$ for fentanyl and 0.517 $\mathrm{ng} / \mathrm{mL}$ for norfentanyl compared with $0.785 \mathrm{ng} / \mathrm{mL}$ and $0.531 \mathrm{ng} / \mathrm{mL}$ in plasma, respectively. ${ }^{27}$

Oral fluid collection generally consists of spitting into vials or using an absorbent material. In order to stabilize the samples, appropriate buffers must be used to house the oral fluid samples. ${ }^{75}$ The Driving Under the Influence of Drugs (DRUID) project in Europe examined the usefulness of oral fluid along with blood samples. This program determined general equivalent cut-off values for 23 substances in oral fluid including codeine $(94 \mathrm{ng} / \mathrm{mL})$, methadone $(22 \mathrm{ng} / \mathrm{mL})$, morphine $(95 \mathrm{ng} / \mathrm{mL})$, and 6-MAM (16 ng/mL). ${ }^{75}$ However, collection performed via stimulation comes with challenges for some drugs and can result in lower drug concentrations than unstimulated saliva. ${ }^{40}$

A major advantage of using oral fluid as a matrix is that the analysis can proceed immediately without additional sample preparation steps. A simple dilute-and-shoot approach (oral fluid diluted in buffer), could be used alone or in combination with other sample preparation schemes involving, for example, solid-phase extraction or liquid-liquid extraction. ${ }^{75} \mathrm{~A}$ crucial aspect to consider is the presence of mucoproteins, which can bind some drugs and interfere with the overall analysis. Interferences by mucin can be alleviated using a cycle of freeze-thaw. Currently, many forensic laboratories use immunoassays as a presumptive, fast drug-class 
Part III: LC/MS/MS Analysis of Fentanyl and its Analogs in Oral Fluid

identification method followed by different mass spectrometric platforms for confirmatory methods employing either GC-MS, GC-MS/MS, LC-MS, or LC-MS/MS. ${ }^{75}$

\subsection{Method Validation: LC/MS}

Method validation is a critical aspect of analytical protocols in order to ensure the reliability and reproducibility of the analyses conducted. Several organizations have written and issued documents detailing their recommendations on how to conduct validation studies and what parameters are essential to consider for various types of analyses. ${ }^{78}$ Validation, as provided by ISO 17025, is the "confirmation by examination and provision of objective evidence that the particular requirements for a specific intended use are fulfilled." ${ }^{79}$ Commonly, the term "fit for purpose"79 is used when discussing method validation or methods in general. Although this term can be vague or arbitrary, Eurachem, the American Academy of Forensic Science Standards Board (ASB), and other entities have attempted to outline parameters that they deem critical to the assessment of the "success" of methods.

\subsubsection{Quality of Results}

The quality of a result can be expressed using various performance measures. Typically, these include bias and precision. ${ }^{79}$ Bias refers to the assessment of the trueness of a measurement. As trueness cannot be measured since it is based on an infinite number of measurements, bias becomes the approximation of this concept and is the difference between the mean value of the results and the known value, expressed as percent bias. ${ }^{79}$ ASB recommends that bias be assessed in triplicate at low, medium, and high concentrations over five different runs using a pooled fortified matrix with a maximum acceptable bias of $\pm 20 \% .{ }^{65}$

Precision provides a measure of how repeatable the method's results are ${ }^{79}$ and is generally expressed as the coefficient of variation:

$$
\% C V=\frac{s}{\bar{x}}(100)
$$

where $\mathrm{s}$ is the standard deviation of the results and $\bar{x}$ is the mean of the results. ${ }^{65}$ Of interest to LC/MS methods are two types of precision that should be assessed: within-run 
precision and between-run precision. It is recommended that these studies be carried out in the same way as the bias studies with a maximum acceptable $\% \mathrm{CV}$ of $\pm 20 \%$.

\subsubsection{Calibration Model and Carryover}

For quantitative procedures, a working range of concentrations is generally determined for analysis. In order to analyze and evaluate samples within this range, it must be shown that the analytical response has a relationship with the analyte concentration. ${ }^{65}$ In general, linear models are used most often, and both the correlation coefficient and residual plots ${ }^{65,78}$ should be used when assessing a model. It should be noted that methods spanning more than one order of magnitude will generally demonstrate heteroscedasticity and be considered candidates for weighted models. ${ }^{78}$ The assessment should be performed on the combined data from 5 replicates of at least six different non-zero calibrators. ${ }^{65}$ Carryover must also be assessed for methods due to the possible contamination of subsequent samples during the analysis process. The procedure for addressing carryover is to analyze a blank sample following high concentrations. It is recommended that carryover be assessed in triplicate for all analytes. ${ }^{65}$

\subsubsection{Interference/Selectivity Studies}

LC/MS methods need to be highly selective for the chosen analytes in the presence of interfering compounds. Interference can affect the method response through signal enhancement or suppression of the signal, and in turn, affect quantitation. ${ }^{79}$ Analysis of interferences can be accomplished in several ways. The first is to demonstrate that there are no interferences from the chosen matrix through the analysis of at least ten different matrix sources. ${ }^{65}$ Commonly encountered substances that may be expected to be present in authentic samples must also be analyzed to determine that they will not interfere with the analysis. ${ }^{78}$ This can be accomplished by analyzing authentic samples or analyzing the compounds using the method. ${ }^{65}$ Lastly, when utilizing isotopically labeled internal standards, it should be demonstrated that the internal standards are free from non-labeled compounds by analyzing the internal standards through the monitoring of the analyte transitions. ${ }^{65}$ 


\subsubsection{Recovery, Matrix Effects, and Process Efficiency}

Recovery (RE) refers to the ability to obtain the analyte after it has undergone sample processing such as solid-phase or liquid-liquid extraction. Although ASB standard 036 does not specifically address recovery, some journals require that recovery be assessed ${ }^{78}$ and is commonplace in method development for LC/MS. Matrix effects (ME), also referred to as ionization suppression or enhancement, is a familiar yet not fully understood phenomenon. ${ }^{78,80}$ Considering LC/MS, matrix effects can be the result of substances that may co-elute with analytes of interest, whether they are also detected or not. ${ }^{80}$ The presence of these components during the ionization process can inhibit or enhance the ionization of the parent molecule, resulting in increased or decreased signal. ${ }^{80}$ Process efficiency (PE) refers to the overall ability of the method to achieve the desired results and is also not specifically addressed in ASB Standard 036 or Eurachem. Matuszewski et al. describe a protocol for the assessment of recovery, matrix effects, and process efficiency. ${ }^{80}$ The procedure describes the preparation of 3 different sets of samples. The first set (A) consisted of neat samples, the second set (B) consisted of processed samples spiked after extraction, and the third set (C) consisted of processed samples spiked before extraction. ${ }^{80}$ The following equations were used to calculate the percent RE, ME, and PE:

$$
\begin{aligned}
& R E=\frac{C}{B} \times 100 \\
& M E=\frac{B}{A} \times 100 \\
& P E=\frac{C}{A} \times 100
\end{aligned}
$$

where the values of RE, ME, and PE are considered "perfect" at 100\%. ${ }^{80}$ However, ASB standard 036 suggests that the calculation of matrix effect be determined from $0 \%$, where positive values refer to ionization enhancement and negative values refer to ionization suppression by subtracting the resulting fraction from $1 .^{65}$ These validation parameters should be assessed at both low and high concentrations using at least 10 sources of matrix, if possible, with matrix effects not exceeding $\pm 25 \%{ }^{65}$ 


\subsubsection{Limit of Detection and Lowest Limit of Quantitation}

The limit of detection (LOD) refers to the lowest concentration where the target analyte can be reliably identified but does not fulfill the requirements for quantification. ${ }^{78}$ This differs from the lowest limit of quantification, which is the lowest concentration of the analyte that can be reliably quantified. ${ }^{78} \mathrm{~A}$ number of methods have been outlined in the literature for the determination of the limit of detection. These include using the lowest non-zero calibrator, estimation through the use of reference materials, and estimation using a linear calibration curve. The LOD, when determined using a linear calibration curve, is calculated through the following equation:

$$
L O D=\frac{3.3 \times s_{y}}{A v g_{m}}
$$

where $S_{y}$ is the standard deviation of the $y$-intercept of at least three calibration curves and $\operatorname{Avg}_{\mathrm{m}}$ is the average slope of the three curves. ${ }^{65}$ The LLOQ can also be assessed using several methods. ${ }^{65}$ However, it is common to report the LLOQ as the lowest non-zero calibrator as it is generally considered inappropriate to perform quantitation outside of the established calibration curve. A rule-of-thumb for assessing the LLOQ includes determining if the signal-to-noise ratio is greater than or equal to $10 .^{78}$ The LLOQ should be assessed in triplicate over three runs. ${ }^{65}$

\subsubsection{Stability}

Many reasons require the stability of samples to be assessed, including storage for subsequent or later analysis such as in forensic science, instrument malfunctions or errors, and storage or holding of processed samples. Therefore, it is necessary to ensure that target analytes will still be detected under the abovementioned conditions. Several reports and guidelines recommend the assessment of processed sample stability in the freezer, refrigerator, or autosampler and assessment of freeze/thaw stability. ${ }^{78}$ It is recommended that stability be measured at low and high concentrations of fortified matrix measured in triplicate. Analysis of samples is performed by comparing the peak area or the peak area ratio of time zero samples to each subsequent stability run. ${ }^{65}$ Time intervals can be chosen to span set times or times commonly 
Part III: LC/MS/MS Analysis of Fentanyl and its Analogs in Oral Fluid

encountered during analysis, and freeze/thaw samples should be assessed over three freeze/thaw cycles. ${ }^{65}$ 


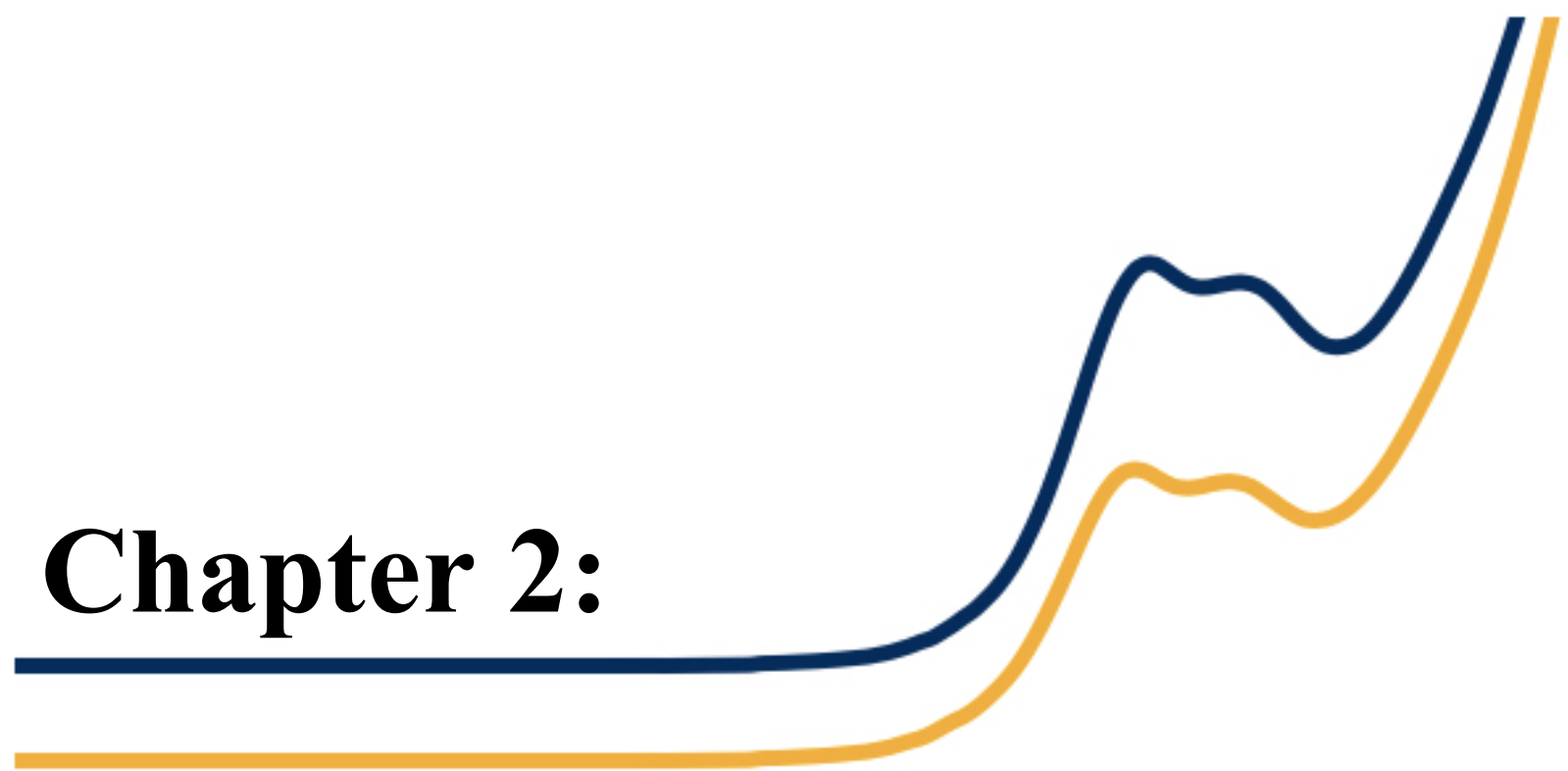

Materials, Methods, and Experimental Design 


\subsection{Materials}

2'-fluoro ortho-fluorofentanyl, 4-ANPP, 4-Fluoroisobutyrl fentanyl, Acetyl fentanyl, Acrylfentanyl, Carfentanil, Despropionyl para-Fluorofentanyl, Fentanyl, Furanyl fentanyl, Methoxyacetyl fentanyl, Norfentanyl, U-47700, Valeryl fentanyl, Morphine, Codeine, Oxycodone, Hydrocodone, 6-monoacetylmorphine, Amphetamine, Ethylmorphine, Methamphetamine, Benzoylecgonine, Norbuprenorphine, Buprenorphine, and Methadone standards, as well as, D5-Norfentanyl, D5-Fentanyl, D5-Acryl fentanyl, D5-Furanyl fentanyl, D5Carfentanil, D6-U-47700, and D5-Valeryl fentanyl internal standards were purchased from Cerilliant (Round Rock, TX).

Methanol (Optima $\left.{ }^{\circledR}\right)$, formic acid (Optima $\left.{ }^{\circledR}\right)$, Ammonium formate, Methylene chloride $\left(\right.$ Optima $\left.{ }^{\circledR}\right)$, 2-propanol (Optima $\left.{ }^{\circledR}\right)$, concentrated Hydrochloric acid (Trace grade) and concentrated Ammonium hydroxide were purchased from Fisher Scientific (Fair Lawn, NJ). Monobasic sodium phosphate, dibasic sodium phosphate, sodium hydroxide, and potassium chloride were purchased from Sigma-Aldrich (St. Louis, MO). A Millipore Direct-Q ${ }^{\circledR}$ UV water purification system (Billerica, MA) was used to obtain purified water (18.2 M $\Omega$ ). Bond Elut Certify, $130 \mathrm{mg} 3 \mathrm{~mL}$ solid-phase extraction (SPE) cartridges were obtained from Agilent (Santa Clara, CA). The oral fluid was provided by non-opioid using volunteers through expectoration and was pooled $(n=7)$ for use with the confirmatory method validation. Blank matrix was analyzed to ensure that the matrix was free from target analytes.

\subsection{Instrumentation}

An Agilent 1290 Infinity II Liquid Chromatography system with an Agilent 6470 Triple Quadrupole Mass Spectrometer (Santa Clara, CA) was utilized for confirmatory analysis with Agilent MassHunter software. Solid-phase extraction was performed utilizing a Positive Pressure Manifold from UCT (Bristol, PA).

\subsection{Preparation of Standard Working Solutions}

Stock solutions of standards and internal standards (ISTD) were prepared in methanol to $100 \mu \mathrm{g} / \mathrm{mL}$ or $10 \mu \mathrm{g} / \mathrm{mL}$. Methanolic calibrator solutions were prepared to $0.1,0.5,1,5,10,25$, 
and $50 \mathrm{ng} / \mathrm{mL}$ containing all standards. ISTD concentration was prepared at $25 \mathrm{ng} / \mathrm{mL}$ in calibrator samples. Negative controls were prepared with only ISTD. All standard solutions were stored at $20{ }^{\circ} \mathrm{C}$ in amber vials.

\subsection{Extraction Procedure}

Fortification or spiking of the mix of standard solutions was utilized to achieve the desired concentrations. ISTD was spiked into the oral fluid samples to achieve $25 \mathrm{ng} / \mathrm{mL}$. Cartridges were conditioned with $1 \mathrm{~mL}$ of methanol followed by $1 \mathrm{~mL}$ of water then $1 \mathrm{~mL}$ of $10 \mathrm{mM}$ phosphate buffered saline (PBS) supplemented with $100 \mathrm{mM} \mathrm{KCl}$. The oral fluid was loaded onto the column for a final volume of $100 \mu \mathrm{L}$. The column was dried under approximately 20 psi nitrogen for 3-5 minutes. Elution was then carried out using $1 \mathrm{~mL}$ of methylene chloride:isopropyl alcohol:ammonium hydroxide (78:20:2). The eluted volume was then evaporated under nitrogen at approximately $45^{\circ} \mathrm{C}$. The samples were reconstituted in $100 \mu \mathrm{L}$ of mobile phase A:mobile phase B (95:5). A total of $1 \mu \mathrm{L}$ was injected on the LC/MS/MS.

\subsection{Liquid Chromatography}

Chromatographic separation was completed using an Agilent RR-HD Zorbax Eclipse Plus C18 column (3.0 x 100 mm, 1.8 microns) with an Agilent SB-C18 pre-column $(3.0 \times 5$ mm, 1.8 microns). The column temperature was maintained at room temperature. A gradient elution at 0.5 $\mathrm{mL} / \mathrm{min}$ with a $0.1 \%$ formic acid and $5 \mathrm{mM}$ ammonium formate in water (mobile phase A) and $0.1 \%$ formic acid in methanol (mobile phase B) was used to achieve separation (Table 6).

\begin{tabular}{|c|c|c|}
\hline Time (min) & A (\%) & B (\%) \\
\hline 0.00 & 95 & 5 \\
\hline 1.00 & 95 & 5 \\
\hline 8.50 & 5 & 95 \\
\hline
\end{tabular}

\subsection{Mass Spectrometry}

Dynamic multiple reaction monitoring (dMRM) was used for data acquisition in positive mode electrospray ionization. A minimum of two transitions per analyte and internal standard were used for analysis (Table 7). Source parameters were optimized using MassHunter Source 
Part III: LC/MS/MS Analysis of Fentanyl and its Analogs in Oral Fluid

Optimizer and were as follows: gas temperature $325^{\circ} \mathrm{C}$, gas flow $5 \mathrm{~L} / \mathrm{min}$, nebulizer 25 psi, sheath gas temperature $350^{\circ} \mathrm{C}$, sheath gas flow $11 \mathrm{~L} / \mathrm{min}$, and capillary voltage $3500 \mathrm{~V}$.

Table 7: Optimized parameters for analysis of analytes by LC/MS/MS.

\begin{tabular}{|c|c|c|c|c|c|c|}
\hline Analyte & $\begin{array}{c}\text { Retention } \\
\text { Time } \\
\text { (min) }\end{array}$ & $\begin{array}{c}\text { Quant } \\
\text { Transition } \\
(\mathrm{m} / \mathrm{z})\end{array}$ & $\begin{array}{c}\text { Qualifier } \\
\text { Transition } \\
(\mathrm{m} / \mathrm{z})\end{array}$ & $\begin{array}{l}\text { Fragmentor } \\
\text { Voltage } \\
\text { (V) }\end{array}$ & $\begin{array}{l}\text { Collision } \\
\text { Energy (V) }\end{array}$ & Internal Standard \\
\hline Norfentanyl & 5.57 & $233-84$ & $\begin{array}{c}233-150 \\
233-55\end{array}$ & 107 & $20,20,44$ & D5-Norfentanyl \\
\hline $\begin{array}{l}\text { Methoxy acetyl } \\
\text { fentanyl }\end{array}$ & 6.00 & $353-188$ & $\begin{array}{c}353-105 \\
353-77\end{array}$ & 92 & $24,44,108$ & D5-Fentanyl \\
\hline Acetyl Fentanyl & 6.13 & $323-105$ & $\begin{array}{c}323-188 \\
323-77\end{array}$ & 98 & $44,24,96$ & D5-Fentanyl \\
\hline 4-ANPP & 6.41 & $281-105$ & $281-188$ & 122 & 36,16 & D5-Fentanyl \\
\hline Acryl Fentanyl & 6.49 & $335-105$ & $\begin{array}{c}335-188 \\
335-77\end{array}$ & 94 & $44,24,94$ & D5-Acryl fentanyl \\
\hline $\begin{array}{l}\text { Despropionyl para- } \\
\text { fluorofentanyl }\end{array}$ & 6.54 & $299-188$ & $\begin{array}{c}299-105 \\
299-77\end{array}$ & 98 & $20,40,88$ & D5-Fentanyl \\
\hline Furanyl Fentanyl & 6.57 & $375-188$ & $375-105$ & 146 & 24,48 & D5-Furanyl fentanyl \\
\hline Fentanyl & 6.61 & $337-188$ & $337-105$ & 146 & 24,48 & D5-Fentanyl \\
\hline Carfentanil & 6.75 & $395-335$ & $\begin{array}{l}395-113 \\
395-105\end{array}$ & 136 & $16,36,56$ & D5-Carfentanil \\
\hline $\begin{array}{l}\text { 2'-fluoro ortho- } \\
\text { fluorofentanyl }\end{array}$ & 6.81 & $373-206$ & $\begin{array}{l}373-123 \\
373-103\end{array}$ & 106 & $28,48,64$ & D5-Fentanyl \\
\hline U-47700 & 6.89 & $329-173$ & $\begin{array}{l}329-145 \\
329-109\end{array}$ & 86 & $36,64,88$ & D6-U-47700 \\
\hline FIBF & 7.04 & $369-188$ & $\begin{array}{c}369-105 \\
369-77\end{array}$ & 92 & $28,48,108$ & D5-Fentanyl \\
\hline Valeryl Fentanyl & 7.43 & $365-188$ & $\begin{array}{c}365-105 \\
365-77\end{array}$ & 100 & $28,48,108$ & D5-Valeryl fentanyl \\
\hline
\end{tabular}

\subsection{Validation}

This method was validated following the recommendations from the American Academy of Forensic Science Standards Board (ASB). Linearity was determined using seven non-zero calibrators. The lowest limit of quantitation (LLOQ) was evaluated as the lowest non-zero calibrator analyzed a total of six times. The limit of detection was determined through serial dilution and evaluated based on signal-to-noise for identification of the quantifier ion. Bias and precision were assessed in pooled oral fluid samples through spiking during SPE. Low (0.5 $\mathrm{ng} / \mathrm{mL})$, medium $(5 \mathrm{ng} / \mathrm{mL})$, and high $(25 \mathrm{ng} / \mathrm{mL})$ concentrations were assessed in triplicate over five runs. Matrix effects and recovery were assessed at low $(0.5 \mathrm{ng} / \mathrm{mL})$ and high $(5 \mathrm{ng} / \mathrm{mL})$ 
concentrations through analysis of sets A, B and C. Set A consisted of neat methanolic samples spiked with standard and ISTD to the desired concentration. Set B consisted of extracted oral fluid samples spiked with standard and ISTD during reconstitution. Set C consisted of extraction of spiked oral fluid samples.

A low and high for set A were each injected a total of 6 times. Sets B and C were analyzed in duplicate with ten replicates each (replicates 1-7 were individual oral fluid matrix, while replicates 8-10 were pooled matrix from the seven donors). Matrix effects, recovery, and process efficiency were assessed as described in equations 5, 6, and 7. Interferences were evaluated by analyzing twelve additional common drugs at $1 \mu \mathrm{g} / \mathrm{mL}$ in methanolic solution. The drugs tested were: Morphine, Codeine, Oxycodone, Hydrocodone, 6-monoacetylmorphine, Amphetamine, Ethylmorphine, Methamphetamine, Benzoylecgonine, Norbuprenorphine, Buprenorphine, and Methadone. Carryover was assessed through the use of a negative QC sample immediately following the highest calibrator in every run. Processed sample stability was assessed for three freeze/thaw cycles using fortified matrix. Autosampler stability was assessed at room temperature over 72 hours. Freezer stability was assessed at $-20^{\circ} \mathrm{C}$ over 72 hours. 
Part III: LC/MS/MS Analysis of Fentanyl and its Analogs in Oral Fluid

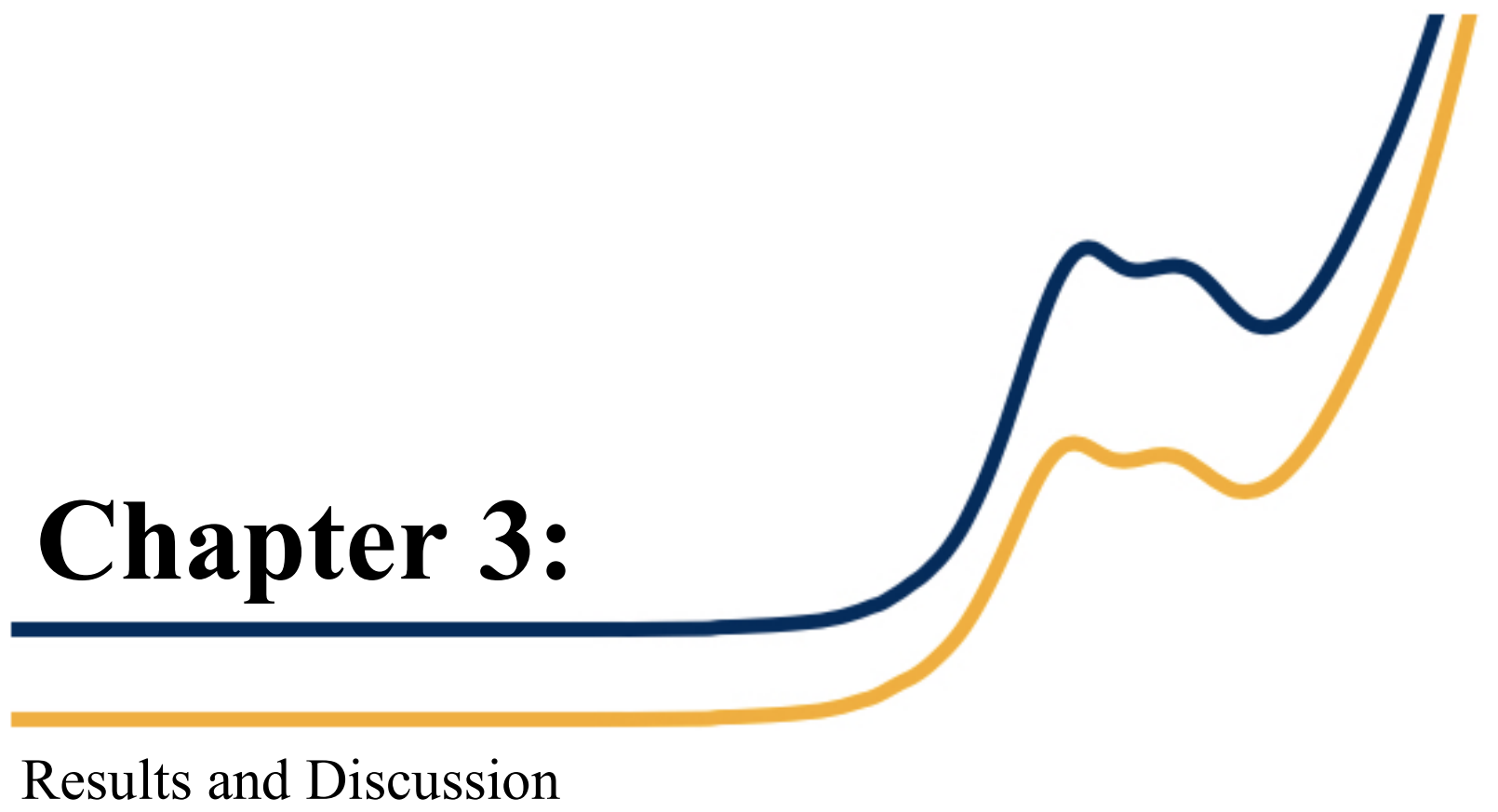




\subsection{Chromatographic Separation}

Chromatographic separation was achieved through the use of the developed dMRM method. No isobaric interferences were present, and acceptable peak resolution was achieved. Figure 35 demonstrates the resolution between the analyte peaks of interest.

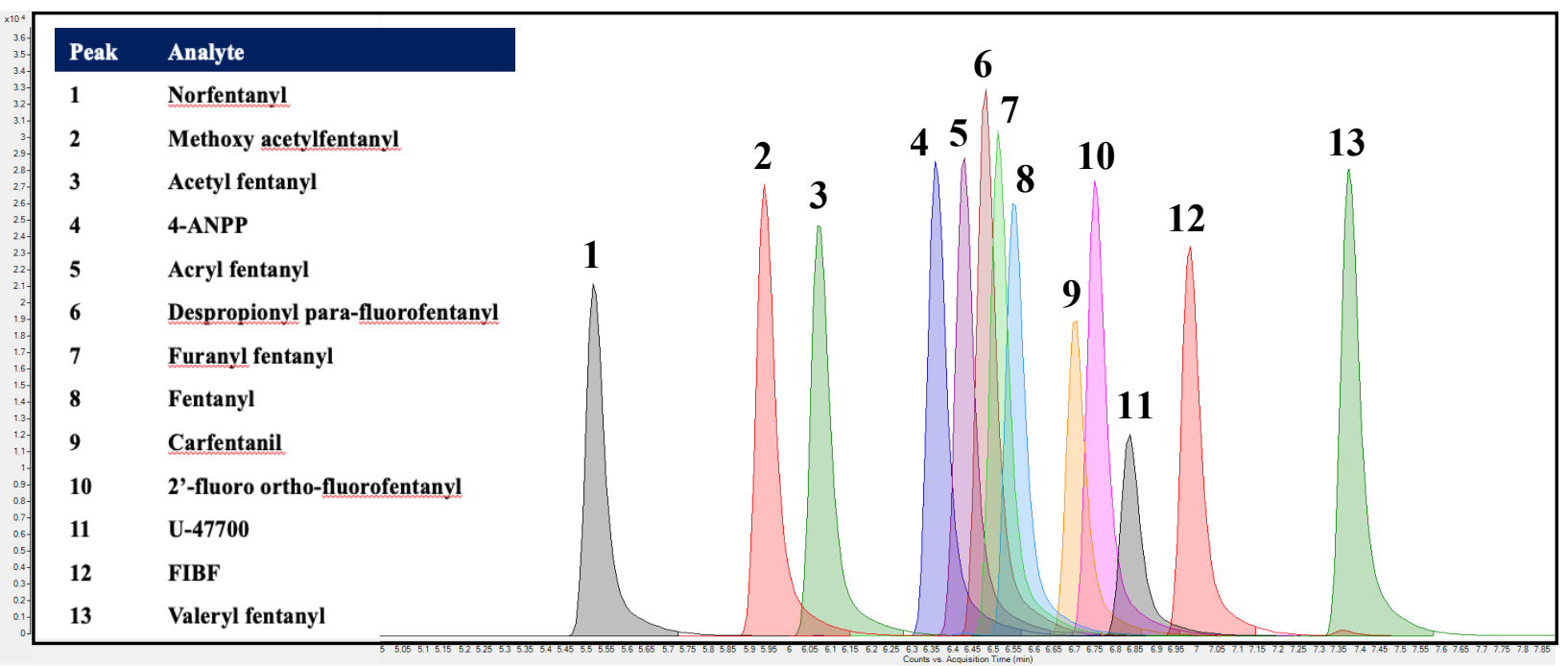

Figure 35: Chromatographic separation of analytes of interest using dMRM.

\subsection{LC/MS/MS Method Validation}

The calibration model was determined to be linear, and all analytes had an $\mathrm{R}^{2}$ value that was $\geq 0.9992$. Weighting of $1 / \mathrm{x}$ was utilized for the calibration range between 0.1 and $50 \mathrm{ng} / \mathrm{mL}$. LODs were within ASB recommendation with signal-to-noise ratios of the quantifying ion greater than 3 at $0.01 \mathrm{ng} / \mathrm{mL}$ for the majority of drugs. LLOQs were within ASB recommendations with signal-to-noise ratios greater than 10 and bias and precision less than $\pm 20 \%$ at $0.1 \mathrm{ng} / \mathrm{mL}$ (Table 8). 
Part III: LC/MS/MS Analysis of Fentanyl and its Analogs in Oral Fluid

Table 8: Linearity, limit of detection (LOD), and lowest limit of quantitation (LLOQ) for the analytes

\begin{tabular}{|c|c|c|c|c|c|}
\hline Analyte & $\begin{array}{c}\mathrm{y} \text {-intercept } \\
(\text { mean } \pm \mathrm{SD}, \mathrm{n}=5)\end{array}$ & $\begin{array}{c}\text { Slope } \\
(\text { mean } \pm \mathrm{SD}, \mathrm{n}=5)\end{array}$ & $\begin{array}{l}\mathrm{R}^{2} \text { range } \\
(\mathrm{n}=5)\end{array}$ & $\begin{array}{c}\mathrm{LOD} \\
(\mathrm{ng} / \mathrm{mL})\end{array}$ & $\begin{array}{l}\mathrm{LLOQ} \\
(\mathrm{ng} / \mathrm{mL})\end{array}$ \\
\hline Norfentanyl & $0.0081 \pm 0.0038$ & $0.0403 \pm 0.0011$ & $\begin{array}{c}0.9999- \\
0.9994\end{array}$ & 0.01 & 0.1 \\
\hline Methoxyacetyl fentanyl & $0.0056 \pm 0.0039$ & $0.0319 \pm 0.0010$ & $\begin{array}{c}0.9999- \\
0.9994\end{array}$ & 0.01 & 0.1 \\
\hline Acetyl Fentanyl & $0.0047 \pm 0.0038$ & $0.0313 \pm 0.0013$ & $\begin{array}{c}0.9999- \\
0.9995\end{array}$ & 0.01 & 0.1 \\
\hline 4-ANPP & $0.0092 \pm 0.0075$ & $0.0531 \pm 0.0013$ & $\begin{array}{c}0.9999- \\
0.9993\end{array}$ & 0.01 & 0.1 \\
\hline Acryl Fentanyl & $0.0105 \pm 0.0057$ & $0.0479 \pm 0.0014$ & $\begin{array}{c}0.9999- \\
0.9993\end{array}$ & 0.01 & 0.1 \\
\hline Despropionyl para-fluorofentanyl & $0.0055 \pm 0.0049$ & $0.0437 \pm 0.0012$ & $\begin{array}{c}0.9999- \\
0.9993\end{array}$ & 0.01 & 0.1 \\
\hline Furanyl Fentanyl & $0.0118 \pm 0.0089$ & $0.0633 \pm 0.0024$ & $\begin{array}{c}0.9999- \\
0.9995\end{array}$ & 0.1 & 0.5 \\
\hline Fentanyl & $0.0071 \pm 0.0057$ & $0.0375 \pm 0.0013$ & $\begin{array}{c}0.9999- \\
0.9992\end{array}$ & 0.01 & 0.1 \\
\hline Carfentanil & $0.0103 \pm 0.0077$ & $0.0614 \pm 0.0019$ & $\begin{array}{c}0.9999- \\
0.9994\end{array}$ & 0.1 & 0.5 \\
\hline 2'-fluoro ortho-fluorofentanyl & $0.0067 \pm 0.0039$ & $0.0342 \pm 0.0011$ & $\begin{array}{c}0.9999- \\
0.9994\end{array}$ & 0.01 & 0.1 \\
\hline U-47700 & $0.0068 \pm 0.0024$ & $0.0366 \pm 0.0009$ & $\begin{array}{c}0.9998- \\
0.9993\end{array}$ & 0.01 & 0.1 \\
\hline FIBF & $0.0047 \pm 0.0037$ & $0.0251 \pm 0.0009$ & $\begin{array}{c}0.9999- \\
0.9992\end{array}$ & 0.01 & 0.1 \\
\hline Valeryl Fentanyl & $0.0094 \pm 0.0055$ & $0.0498 \pm 0.0013$ & $\begin{array}{c}0.9999- \\
0.9996\end{array}$ & 0.01 & 0.1 \\
\hline
\end{tabular}

Bias and precision data are summarized in Table 9. Bias ranged between -15 to $6 \%$ for the low, -15 to $0.5 \%$ for the medium, and -11 to $6 \%$ for the high concentration except for Acetyl fentanyl and Methoxyacetyl fentanyl. Within-run precision was calculated as the percent coefficient of variation ( $\% \mathrm{CV})$ and ranged from 4 to $24 \%$ for the low, 4 to $25 \%$ for the medium, 7 to $31 \%$ for the high concentration. Several drugs were above the acceptable $20 \%$ CV (4-ANPP, Despropionyl para-fluorofentanyl at medium and high, Methoxyacetyl fentanyl at high, and Norfentanyl at low and medium). Between-run precision was assessed as \% $\mathrm{CV}$ and ranged from 6 to $23 \%$ for the low, 4 to $20 \%$ for the medium, and 5 to $23 \%$ for the high concentration. Two drugs were above the acceptable 20\% CV (Despropionyl para-fluorofentanyl for low and high and Norfentanyl for the medium concentration). 
Table 9: Bias and precision data for the analytes tested

\begin{tabular}{|c|c|c|c|c|c|c|c|c|c|}
\hline \multirow[t]{2}{*}{ Analyte } & \multicolumn{3}{|c|}{$\begin{array}{c}\text { Accuracy, Bias }(\%) \\
(\mathrm{n}=15)\end{array}$} & \multicolumn{3}{|c|}{$\begin{array}{l}\text { Within-run Precision } \\
(\% \mathrm{CV})(\mathrm{n}=3)\end{array}$} & \multicolumn{3}{|c|}{$\begin{array}{c}\text { Between-run Precision } \\
(\% \mathrm{CV})(\mathrm{n}=15)\end{array}$} \\
\hline & LOW & MED & HIGH & LOW & MED & HIGH & LOW & MED & $\mathrm{HIGH}$ \\
\hline $\begin{array}{l}\text { 2'fluoro ortho- } \\
\text { fluorofentanyl }\end{array}$ & 5.4 & -3.0 & -8.1 & 16 & 11 & 12 & 9.4 & 7.9 & 10 \\
\hline 4-ANPP & -7.4 & -5.4 & 6.1 & 24 & 21 & 29 & 19 & 17 & 19 \\
\hline 4-Fluoroisobutyrl fentanyl & -7.4 & -11 & -11 & 4.1 & 5.5 & 10 & 6.4 & 3.9 & 7.9 \\
\hline Acetyl fentanyl & 33 & 23 & 27 & 13 & 12 & 17 & 11 & 8.4 & 9.1 \\
\hline Acryl fentanyl & 3.5 & -0.6 & -0.7 & 8.0 & 4.3 & 7.3 & 7.5 & 4.2 & 4.6 \\
\hline Carfentanil & 5.4 & -0.2 & -1.9 & 13 & 6.7 & 8.5 & 9.9 & 4.7 & 6.0 \\
\hline $\begin{array}{l}\text { Despropionyl para- } \\
\text { fluorofentanyl }\end{array}$ & -13 & -15 & -1.4 & 14 & 25 & 31 & 23 & 19 & 23 \\
\hline Fentanyl & 4.3 & -0.3 & -1.2 & 16 & 5.8 & 9.1 & 9.9 & 3.9 & 5.6 \\
\hline Furanyl fentanyl & 3.3 & -1.3 & -2.6 & 11 & 5.5 & 6.9 & 6.3 & 4.3 & 5.1 \\
\hline Methoxy acetyl fentanyl & 44 & 33 & 40 & 13 & 15 & 26 & 11 & 11 & 14 \\
\hline Norfentanyl & -15 & -7.4 & 3.7 & 22 & 21 & 18 & 16 & 20 & 12 \\
\hline U-47700 & 0.3 & 0.5 & -0.8 & 11 & 9.5 & 9.1 & 9.0 & 6.0 & 6.3 \\
\hline Valeryl fentanyl & 5.9 & -1.5 & -3.0 & 14 & 11 & 11 & 10 & 5.8 & 6.1 \\
\hline
\end{tabular}

Matrix effects, recovery, and process efficiency are presented in Table 10. All analytes were within the required $\pm 25 \%$ for matrix effects with the exception of Despropionyl parafluorofentanyl. However, Depropionyl para-fluorofentanyl was shown to have an acceptable bias at low, medium, and high concentrations, although precision suffered slightly. Recovery was greater than $87 \%$ for all analytes. Process efficiency was above $71 \%$ for all analytes and above $80 \%$ for analytes that met matrix effect parameters. Matrix effects were, in general, positive, demonstrating ion enhancement for the majority of analytes. 
Part III: LC/MS/MS Analysis of Fentanyl and its Analogs in Oral Fluid

Table 10: Matrix effect, recovery, and process efficiency for analytes tested

\begin{tabular}{|c|c|c|c|c|c|c|}
\hline \multirow[t]{3}{*}{ Compound } & \multicolumn{2}{|c|}{ Matrix Effects ${ }^{\mathrm{a}}$} & \multicolumn{2}{|c|}{ Recovery } & \multicolumn{2}{|c|}{ Process Efficiency } \\
\hline & LOW & HIGH & LOW & HIGH & LOW & $\mathrm{HIGH}$ \\
\hline & Mean $^{\mathrm{b}}$ & Mean & Mean & Mean & Mean & Mean \\
\hline $\begin{array}{l}\text { 2'fluoro ortho- } \\
\text { fluorofentanyl }\end{array}$ & -3.0 & -6.5 & 90 & 89 & 87 & 84 \\
\hline 4-ANPP & -15 & -22 & 116 & 125 & 99 & 98 \\
\hline $\begin{array}{l}\text { 4-Fluoroisobutyrl } \\
\text { fentanyl }\end{array}$ & -12 & -8.0 & 96 & 87 & 85 & 80 \\
\hline Acetyl fentanyl & 19 & 15 & 107 & 107 & 127 & 123 \\
\hline Acryl fentanyl & 1.1 & 0.8 & 96 & 97 & 97 & 97 \\
\hline Carfentanil & 0.7 & 1.7 & 101 & 98 & 102 & 99 \\
\hline $\begin{array}{l}\text { Despropionyl } \\
\text { para- } \\
\text { flurorofentanyl }\end{array}$ & -27 & -31 & 98 & 114 & 71 & 79 \\
\hline Fentanyl & 1.1 & 0.6 & 100 & 100 & 101 & 100 \\
\hline Furanyl fentanyl & 0.02 & 0.06 & 101 & 97 & 101 & 97 \\
\hline $\begin{array}{l}\text { Methoxy acetyl } \\
\text { fentanyl }\end{array}$ & 25 & 21 & 110 & 110 & 137 & 134 \\
\hline Norfentanyl & 3.0 & 1.2 & 105 & 105 & 109 & 107 \\
\hline U-47700 & -2.7 & -0.6 & 106 & 103 & 103 & 103 \\
\hline Valeryl fentanyl & -0.7 & 1.6 & 105 & 96 & 104 & 97 \\
\hline
\end{tabular}

Selectivity and interference were assessed through the analysis of other drugs of abuse commonly encountered in the forensic laboratory. No interference with the target analytes was seen (Figure 36). No interference from the deuterated internal standards was evident in the analysis. 
Chapter 3: Results and Discussion

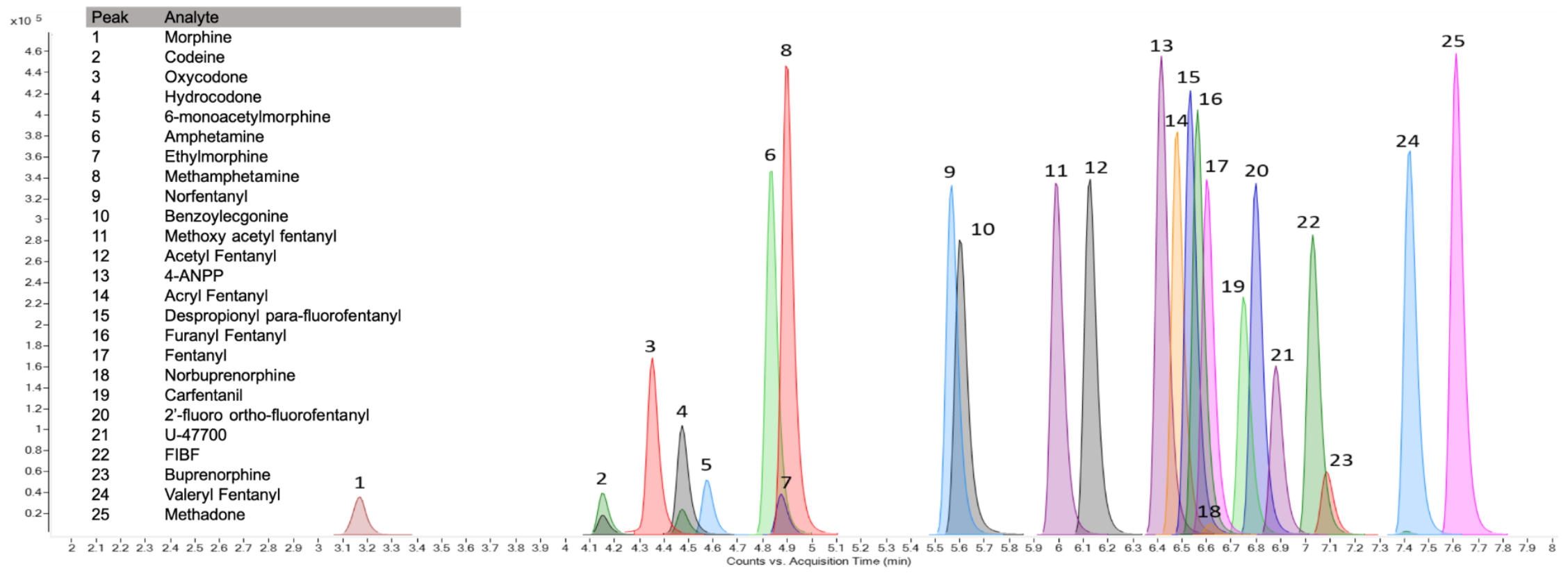

Figure 36: Chromatographic separation of 25 drugs of abuse, including the 13 target analytes. 
Part III: LC/MS/MS Analysis of Fentanyl and its Analogs in Oral Fluid

Freeze/Thaw stability was assessed for three freeze/thaw cycles. Relative response to the internal standard was used to compare to the time zero samples. Pooled, fortified oral fluid was aliquoted and tested for time zero and frozen for 24 hours. Unassisted thaw at room temperature was used to thaw the samples prior to analysis. All analytes were stable within $20 \%$ of time zero, except for 4-ANPP and Despropionyl para-fluorofentanyl (at the high concentration). 2'-fluoro ortho-fluorofentanyl (at the high concentration), Furanyl fentanyl (at the low concentration), Methoxyacetyl fentanyl, and Valeryl fentanyl (at the low concentration) fell outside the 20\% threshold before 72 hours. Table 11 shows the stability of the analytes through three freeze/thaw cycles over 72 hours, assessed as the percent difference from time zero.

Table 11: Freeze/thaw stability for three cycles over 72 hours for pooled fortified matrix

\begin{tabular}{|c|c|c|c|c|c|c|}
\hline \multirow[t]{3}{*}{ Compound } & \multicolumn{2}{|c|}{ Thaw 1} & \multicolumn{2}{|c|}{ Thaw 2} & \multicolumn{2}{|c|}{ Thaw 3} \\
\hline & LOW & HIGH & LOW & HIGH & LOW & $\mathrm{HIGH}$ \\
\hline & Mean & Mean & Mean & Mean & Mean & Mean \\
\hline $\begin{array}{l}\text { 2'fluoro ortho- } \\
\text { fluorofentanyl }\end{array}$ & -9.6 & 3.7 & -1.8 & 28 & -0.6 & 21 \\
\hline 4-ANPP & -31 & -47 & -21 & -37 & -27 & -49 \\
\hline $\begin{array}{l}\text { 4-Fluoroisobutyrl } \\
\text { fentanyl }\end{array}$ & -8.7 & 0.1 & -3.2 & 18 & 5.9 & 3.5 \\
\hline Acetyl fentanyl & -4.7 & -17 & -7.0 & -19 & -10 & -19 \\
\hline Acryl fentanyl & -5.3 & 0.09 & -1.8 & 7.1 & -0.2 & -0.5 \\
\hline Carfentanil & -3.5 & 1.6 & -0.3 & 5.1 & -2.0 & -2.0 \\
\hline $\begin{array}{l}\text { Despropionyl } \\
\text { para- } \\
\text { flurorofentanyl }\end{array}$ & -7.7 & -47 & 9.2 & -35 & 4.2 & -49 \\
\hline Fentanyl & 2.9 & -3.6 & 6.1 & 1.0 & 3.4 & -3.5 \\
\hline Furanyl fentanyl & -15 & -3.2 & -19 & 2.1 & -21 & -6.7 \\
\hline $\begin{array}{l}\text { Methoxy acetyl } \\
\text { fentanyl }\end{array}$ & -7.0 & -20 & -16 & -26 & -18 & -24 \\
\hline Norfentanyl & -7.6 & -0.09 & -9.4 & 7.8 & 4.8 & 10 \\
\hline U-47700 & -10 & -11 & -7.1 & -3.0 & -13 & -5.2 \\
\hline Valeryl fentanyl & -16 & -5.0 & -19 & -1.8 & -28 & -6.0 \\
\hline
\end{tabular}


Processed sample stability was assessed at room temperature storage in the autosampler and storage at $-20^{\circ} \mathrm{C}$ for 24,48 , and 72 hours as the percent difference from time zero (Tables 12 and 13). Again, 4-ANPP and Despropionyl para-fluorfentanyl were not stable as processed samples in $100 \mu \mathrm{L}$, and Acryl fentanyl (at the low concentration) was not stable at the 72-hour mark at room temperature. Furanyl fentanyl and Valeryl fentanyl fell outside of $20 \%$ for the low concentration at 48 hours but were below $20 \%$ at 72 hours. Therefore, it was considered that these analytes were stable. All analytes were stable for 72 hours with freezer storage at $-20^{\circ} \mathrm{C}$ except Despropionyl para-fluorofentanyl (at the low concentration). Plots demonstrating the stability of all 13 analytes under the various conditions can be found in Figure 37. Individual analyte stability plots can be found in the appendix, which demonstrate the stability of each analyte along with the $\pm 20 \%$ threshold lines (Figures A5-A7).

Table 12: Room temperature autosampler stability of analytes for processed samples over 72 hours in the fortified, pooled matrix

\begin{tabular}{|c|c|c|c|c|c|c|}
\hline \multirow[t]{3}{*}{ Compound } & \multicolumn{2}{|c|}{24 hours } & \multicolumn{2}{|c|}{48 hours } & \multicolumn{2}{|c|}{72 hours } \\
\hline & LOW & HIGH & LOW & $\mathrm{HIGH}$ & LOW & $\mathrm{HIGH}$ \\
\hline & Mean $^{b}$ & Mean & Mean & Mean & Mean & Mean \\
\hline $\begin{array}{l}\text { 2'fluoro ortho- } \\
\text { fluorofentanyl }\end{array}$ & 3.3 & 11 & 12 & 2.7 & 6.2 & 6.7 \\
\hline 4-ANPP & -21 & -24 & -26 & -27 & -51 & -28 \\
\hline $\begin{array}{l}\text { 4-Fluoroisobutyrl } \\
\text { fentanyl }\end{array}$ & 1.5 & -2.4 & 14 & -3.7 & -2.3 & -6.4 \\
\hline Acetyl fentanyl & -3.5 & -8.9 & -1.2 & -7.1 & 0.9 & -7.0 \\
\hline Acryl fentanyl & 1.0 & -1.0 & -0.9 & -0.3 & -32 & 1.0 \\
\hline Carfentanil & -0.4 & 1.4 & -2.9 & -0.6 & -1.9 & -2.6 \\
\hline $\begin{array}{l}\text { Despropionyl } \\
\text { para- } \\
\text { flurorofentanyl }\end{array}$ & 3.3 & -28 & -7.0 & -29 & -38 & -34 \\
\hline Fentanyl & 4.9 & -3.5 & 4.8 & -5.1 & 5.5 & -4.0 \\
\hline Furanyl fentanyl & -6.9 & -0.1 & -32 & -3.4 & -9.8 & -0.6 \\
\hline $\begin{array}{l}\text { Methoxy acetyl } \\
\text { fentanyl }\end{array}$ & 0.07 & -6.8 & -0.9 & -4.0 & 6.3 & -3.6 \\
\hline Norfentanyl & -3.0 & -2.0 & -4.4 & -0.9 & -4.6 & -1.8 \\
\hline $\mathrm{U}-47700$ & -3.7 & -1.3 & -3.4 & 0.08 & -8.7 & -6.5 \\
\hline Valeryl fentanyl & -19 & -2.7 & -27 & -3.9 & -19 & -5.3 \\
\hline
\end{tabular}


Part III: LC/MS/MS Analysis of Fentanyl and its Analogs in Oral Fluid

Table 13: Freezer stability of analytes for processed samples over 72 hours in the fortified, pooled matrix

\begin{tabular}{|c|c|c|c|c|c|c|}
\hline \multirow[t]{3}{*}{ Compound } & \multicolumn{2}{|c|}{24 hours } & \multicolumn{2}{|c|}{48 hours } & \multicolumn{2}{|c|}{72 hours } \\
\hline & LOW & HIGH & LOW & HIGH & LOW & $\mathrm{HIGH}$ \\
\hline & Mean $^{\mathrm{b}}$ & Mean & Mean & Mean & Mean & Mean \\
\hline $\begin{array}{l}\text { 2'fluoro ortho- } \\
\text { fluorofentanyl }\end{array}$ & 8.2 & 2.6 & 11 & 2.0 & 8.8 & 3.3 \\
\hline 4-ANPP & -13 & -7.8 & -19 & -10 & 19 & -8.9 \\
\hline $\begin{array}{l}\text { 4-Fluoroisobutyrl } \\
\text { fentanyl }\end{array}$ & 4.7 & -4.1 & 0.07 & -13 & 13 & -9.0 \\
\hline Acetyl fentanyl & 1.9 & 3.9 & 1.3 & 5.9 & 6.9 & 8.7 \\
\hline Acryl fentanyl & -1.9 & -0.3 & 2.0 & -0.5 & 0.4 & 0.6 \\
\hline Carfentanil & -13 & -0.5 & -2.5 & -4.0 & 0.5 & 0.8 \\
\hline $\begin{array}{l}\text { Despropionyl } \\
\text { para- } \\
\text { flurorofentanyl }\end{array}$ & -47 & -15 & -45 & -20 & -93 & -20 \\
\hline Fentanyl & -1.3 & -2.1 & -2.6 & -2.4 & 2.0 & -1.7 \\
\hline Furanyl fentanyl & -0.2 & -0.3 & 4.7 & -1.4 & -1.7 & -0.6 \\
\hline $\begin{array}{l}\text { Methoxy acetyl } \\
\text { fentanyl }\end{array}$ & 7.2 & 8.8 & 11 & 13 & 16 & 15.6 \\
\hline Norfentanyl & 1.8 & -0.2 & -1.4 & 0.9 & 3.9 & 1.9 \\
\hline U-47700 & -8.9 & 1.9 & -7.5 & 4.7 & -0.5 & 4.1 \\
\hline Valeryl fentanyl & -6.6 & 1.5 & -9.1 & 3.1 & 5.9 & 2.5 \\
\hline
\end{tabular}
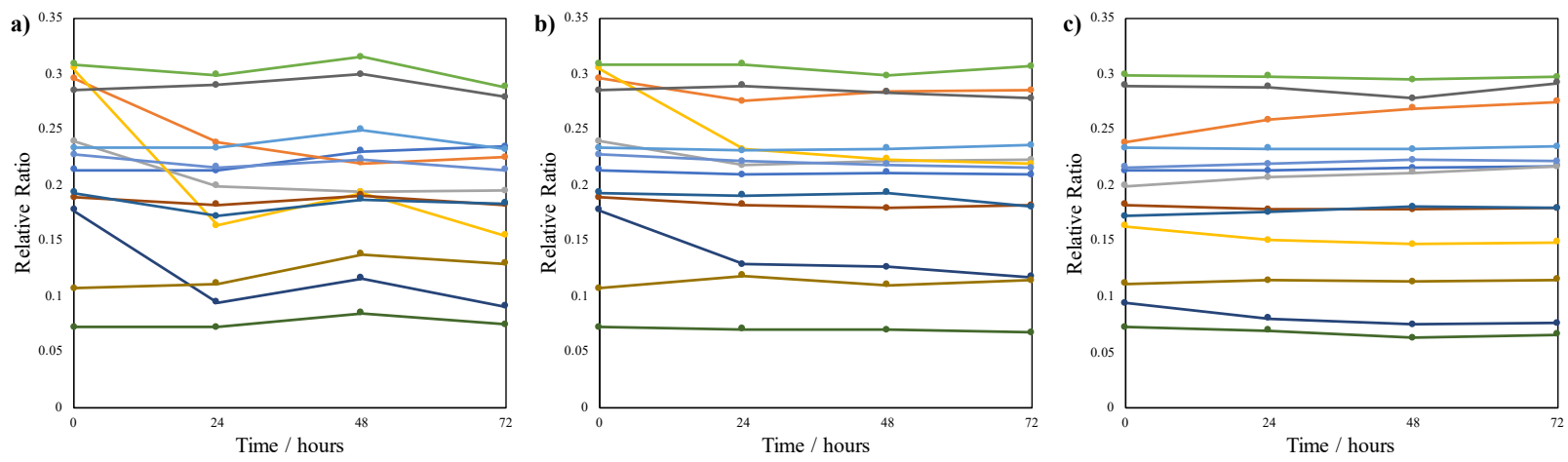

$$
\begin{array}{ll}
\rightarrow \text {-Norfentanyl } & \rightarrow \text {-Methoxyacetyl fentanyl } \\
\rightarrow \text {-Acryl fentanyl } & \rightarrow \text {-Furanyl fentanyl } \\
\rightarrow \text {-Carfentanil } & \rightarrow \text {-'-fluoro ortho-fluorofent }
\end{array}
$$$$
\text { -Acetyl fentanyl }
$$$$
\text { -Despropionyl para-fluorofentanyl }
$$

$\rightarrow-2$-fluoro ortho-fluorofentanyl

$\rightarrow$ U-47700

$\rightarrow$ FIBF

Figure 37: Graphical depiction of the stability of all analytes over 72 hours for freeze/thaw (a), autosampler at room temperature (b), and freezer at $-20^{\circ} \mathrm{C}(\mathrm{c})$. 


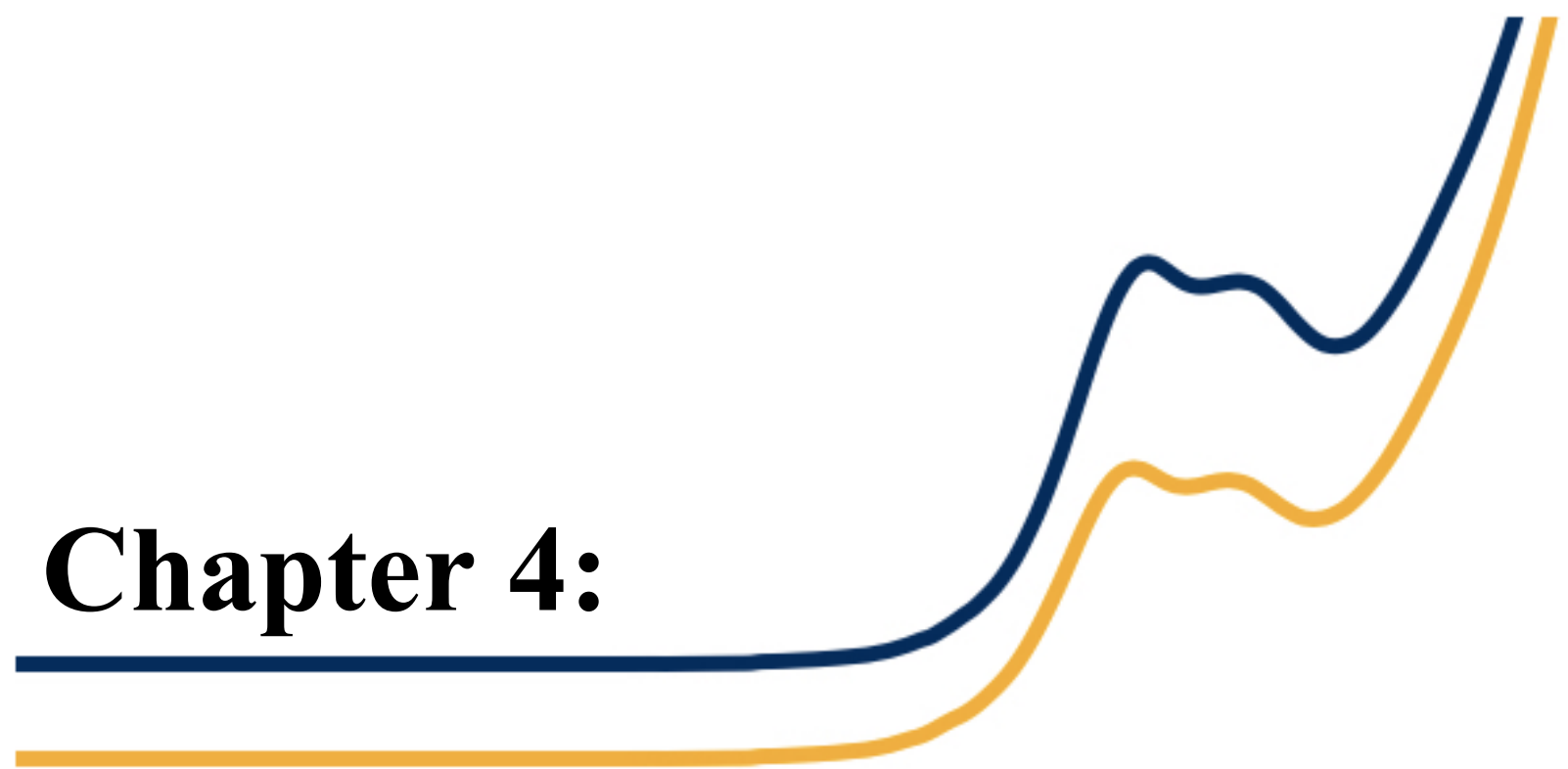

Conclusions and Future Work 


\subsection{Conclusions}

Chromatographic separation was achieved for the panel of target drugs without interference from common drugs of abuse. Several regions of slight co-elution were observed but did not pose any issue as the precursor ions were different. Limits of detection were determined by serial dilution of a standard mix and the assessment of a minimum of 5 calibration curves. The LODs were determined to be $0.01 \mathrm{ng} / \mathrm{mL}$ for all target analytes except Carfentanil and Furanyl fentanyl, which had LODs between $0.01 \mathrm{ng} / \mathrm{mL}$ and $0.1 \mathrm{ng} / \mathrm{mL}$. The majority of the analytes were within acceptable bias and precision limits as specified by the ASB Standard 036. ${ }^{65}$ A solid-phase extraction protocol was explored using spiked oral fluid samples. The wash step was eliminated and resulted in an average recovery for the fentanyl analogs of 102\%. Lastly, stability was assessed over three freeze/thaw cycles, 72 hours of processed freezer storage, and 72 hours of processed autosampler storage. The majority of analytes were shown to be stable under all conditions.

\subsection{Future Work}

Future work on detection of fentanyl using the LC/MS/MS method will include the analysis of more fentanyl analog compounds to expand the method and detection capabilities. Work should also be conducted on authentic oral fluid samples to determine the usefulness of the method on casework-like samples. 


\section{Part IV: Overall Conclusions}

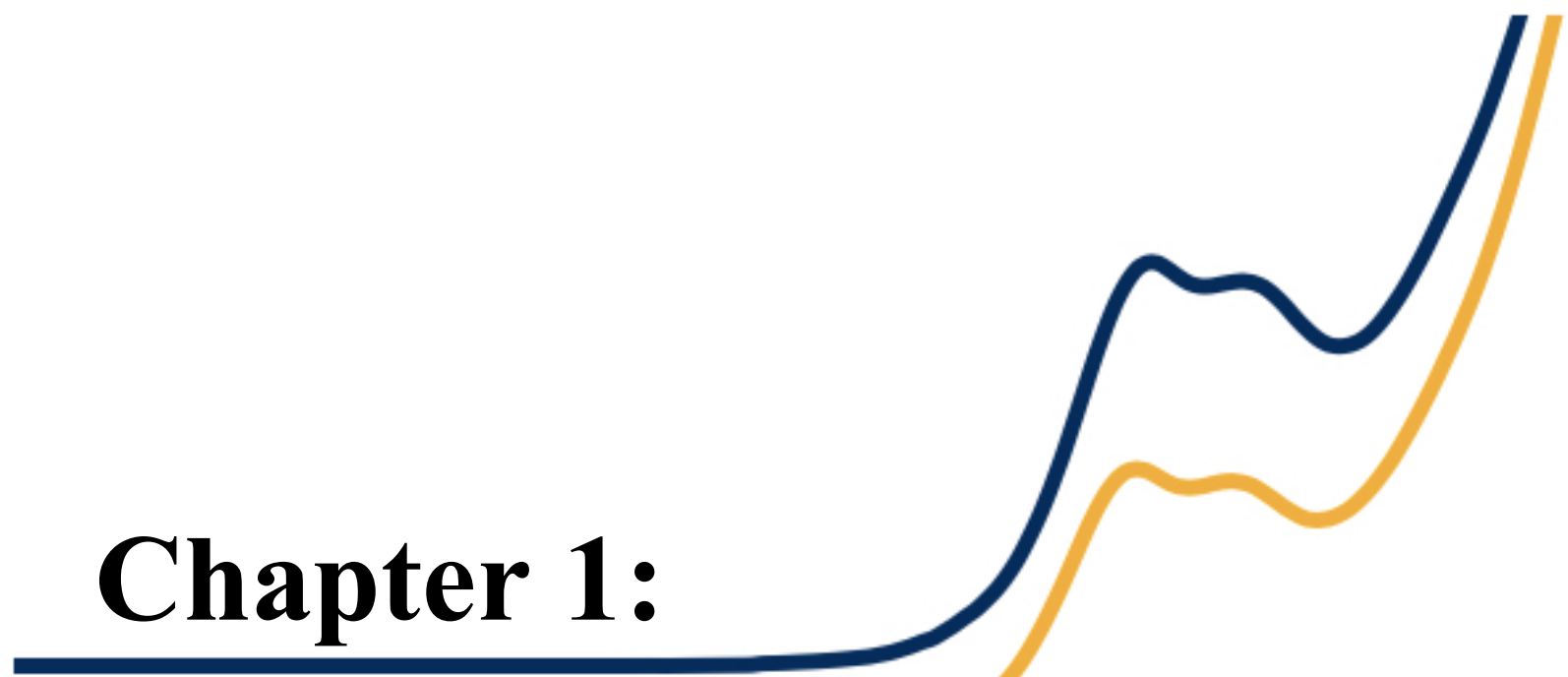

Overall Conclusions 


\subsection{Overall Conclusions}

This thesis demonstrated the use of an electrochemical detection method for fentanyl capable of performing detection measurements in cell and on small sample sizes such as a drop. This approach represents a fast, simple, and cost-effective strategy for the detection of fentanyl in seized drug samples or unknowns. Detection capability was shown to be achieved in the low partsper-billion range with minimal interferences from other commonly encountered drugs. The electrochemical method demonstrates excellent promise for the detection of not only fentanyl, but other illicit drugs as well.

An LC/MS/MS method was also presented for the confirmatory analysis of fentanyl and its analogs in oral fluid. This dMRM method followed validation protocol to describe the identification and quantitation of 13 fentanyl and fentanyl-related compounds, at sub-parts-perbillion levels, without interference from other commonly analyzed drugs. Use of the oral fluid matrix is desirable due to the non-invasive collection of the sample and information regarding recent drug use.

Together the two methods represent an identification method for fentanyl that could be utilized within the laboratory or in the field with the capability of performing quasi-quantitation and a confirmatory method through liquid chromatography-tandem mass spectrometry, validated for use in oral fluid samples. The combined use of these techniques seeks to emulate the SWGDRUG requirement for a category A with a category A, B, or C technique, although electrochemistry has, to this point, not been included in the list of acceptable techniques. This thesis seeks to advance the scientific knowledge regarding the detection of fentanyl and the use of electrochemistry for the detection of drugs of abuse. Implementation of electrochemical techniques in laboratories or in the field could provide a solution to reducing the backlog of cases due, in part, to the opioid epidemic within the country, and could provide a more cost-effective and straightforward screening approach. 


\section{Part V: Other \\ Electrochemical \\ $\underline{\text { Methods }}$}

Chapter 1:

Other Methods 
Several other electrochemical methods were employed during the course of this study to either become more proficient at the techniques and methods utilized, to attempt detection of fentanyl using another electrochemical method, or for the analysis of different compounds. These methods are summarized in brief within this chapter.

\subsection{Enzymatic Biosensor for the Detection of Codeine in Preparation for Fentanyl}

The development of an enzymatic biosensor for fentanyl was initially investigated based on reports of enzymatic modifications providing excellent sensitivity and selectivity for their target analytes. Initial work focused on the reproduction of a codeine sensor developed by colleagues in Spain using cytochrome P450 2D6 (CYP2D6). ${ }^{19}$ The work then focused on the development of a fentanyl sensor using SPEs modified with cytochrome P450 3A4 (CYP3A4).

\subsubsection{Materials and Methods}

Codeine was purchased from Cayman Chemical Company (Ann Arbor, MI). 200 proof ethanol was purchased from Decon Laboratories. Acetonitrile (Optima $\left.{ }^{\circledR}\right)$ was purchased from Fisher Scientific (Fair Lawn, NJ). Sodium hydroxide, potassium chloride, 4nitrobenzenediazonium tetrafluoroborate salt, tetrabutylammonium tetrafluoroborate, $N$ hydroxysuccinimide (NHS), $N$-(3-dimethylaminopropyl)- $N$ '-ethylcarbodiimide $\quad$ (EDC), Monobasic sodium phosphate, and dibasic sodium phosphate were purchased from Sigma Aldrich (St. Louis, MO). Cytochrome P450 2D6 was purchased from U.S. Biological.

Screen-printed carbon electrodes (SPCEs) were obtained from the Universidad de Burgos analytical chemistry department. SPCEs were fabricated using a DEK 248 screen-printing system (DEK, Weymouth, UK) with polyester screens with stencils. SPCEs contained conductive silver tracks, $\mathrm{Ag} / \mathrm{AgCl}$ pseudo-reference electrode, and carbon working $\left(0.126 \mathrm{~cm}^{2}\right)$ and counter electrodes.

A $4 \mathrm{mM}$ diazonium salt solution was prepared by adding 4-nitrobenzenediazonium tetrafluoroborate to $100 \mathrm{mM}$ tetrabutylammonium tetrafluoroborate in acetonitrile. A 9:1, 
water:ethanol mixture was prepared and supplemented with $100 \mathrm{mM} \mathrm{KCl}$. A solution of $40 \mathrm{mM}$ NHS as well as a solution of $80 \mathrm{mM}$ EDC was prepared in $10 \mathrm{mM}$ phosphate buffer $\mathrm{pH} 4$. Lastly, $100 \mathrm{mM}$ phosphate buffer $\mathrm{pH} 7$ was prepared and used as the supporting electrolyte.

Modification of SPCEs through covalent attachment of CYP2D6 for analysis of codeine was performed in a 3-step immobilization procedure. A self-assembling monolayer (SAM) was created through the addition of a $50 \mu \mathrm{L}$ drop of the diazonium salt solution Cyclic voltammetry was then performed between $800 \mathrm{mV}$ and $-400 \mathrm{mV}$ at a scan rate of $200 \mathrm{mV} / \mathrm{sec}$. for two cycles. A $100 \mathrm{uL}$ drop of 9:1 (water:ethanol) was placed on the electrode surface followed by cyclic voltammetry between $0 \mathrm{~V}$ and $-1700 \mathrm{mV}$ at a rate of $200 \mathrm{mV} / \mathrm{sec}$. for two cycles. Lastly, the electrode was washed with the $\mathrm{pH} 4$ phosphate buffer and 0.5 to $1 \mu \mathrm{L}$ of the CYP2D6 enzyme solution was placed on the working electrode followed by $2 \mu \mathrm{L}$ of NHS and $1 \mu \mathrm{L}$ of EDC. The electrode was then left at $4^{\circ} \mathrm{C}$ for 90 minutes. These concentrations and volumes were adjusted to explore if better signal could be obtained. The modification approach is demonstrated in Figure 37.

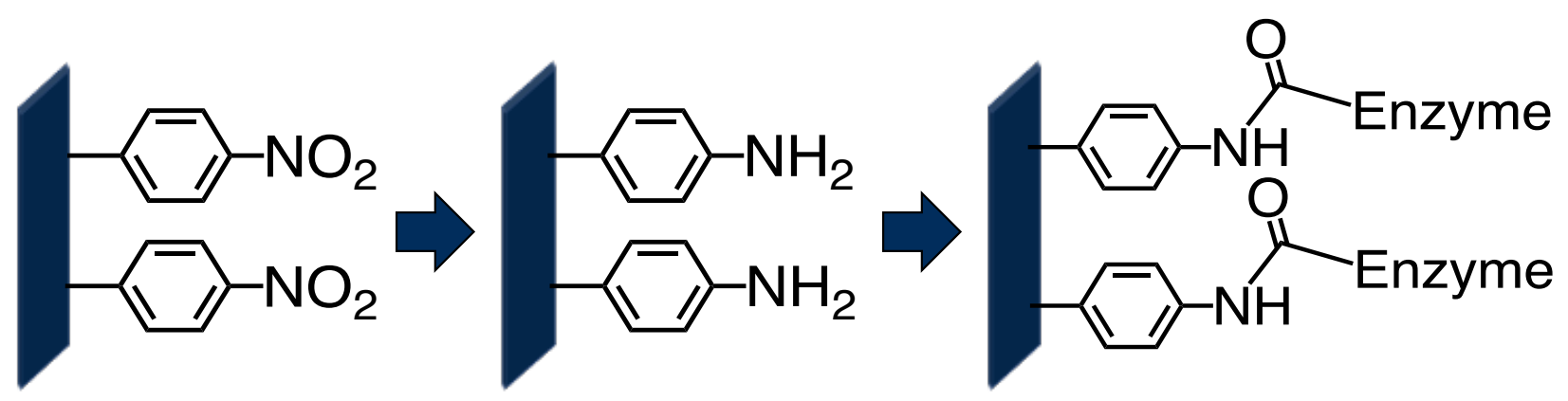

Figure 38: Covalent attachment strategy via self-assembled monolayer for CYP2D6 on carbon surface of SPCE.

Codeine $(1 \mathrm{mg})$ was prepared in $100 \mathrm{mM}$ phosphate buffer $\mathrm{pH} 7$ to a stock solution of 1 $\mathrm{mg} / \mathrm{mL}(1000 \mu \mathrm{g} / \mathrm{mL})$. Further concentrations were prepared as needed. Analysis of codeine was conducted in a $5 \mathrm{~mL}$ electrochemical cell containing $100 \mathrm{mM}$ phosphate buffer $\mathrm{pH} 7$ and as a drop on the electrode surface using chronoamperometry through the application of $200 \mathrm{mV}$ in a stirred solution. After allowing the background current to equilibrate, standard additions of codeine were added to the cell at equal time intervals and the current was recorded. Analysis of codeine was also conducted through addition to a $150 \mu \mathrm{L}$ drop. 


\subsubsection{Instrumentation}

Electrochemical measurements were carried out using the PalmSens4 potentiostat with PSTrace software (Randhoeve, Netherlands) and Autolab PGSTAT with NOVA software from Metrohm (Ionenstrasse, Switzerland). Measurement of solution $\mathrm{pH}$ was achieved using a Mettler Toledo FiveEasy Plus pH meter (Columbus, OH). A Millipore Direct-Q® UV water purification system (Billerica, MA) was used to obtain purified water.

\subsubsection{Results and Discussion}

The first step in the covalent attachment of the enzyme represents the attachment of the diazonium salt to the carbon working electrode. This was followed by the reduction of the nitro groups to amine groups allowing for the covalent attachment of the enzyme to the amine group. The resulting cyclic voltammograms for the attachment can be seen in Figure 38. These were in partial agreement with the literature. Where the literature suggests that there should be a large reduction peak resulting from the nitro group reduction around $-1.2 \mathrm{~V}$, only a small wave was observed. The enzyme modification was checked using cyclic voltammetry in the supporting electrolyte and was found to be different than presented in the literature, although for a different CYP enzyme (Figure 39). ${ }^{17}$ This could reflect a problem encountered during the covalent attachment of the enzyme, suggesting that the enzyme may not be adequately bound or is missing.
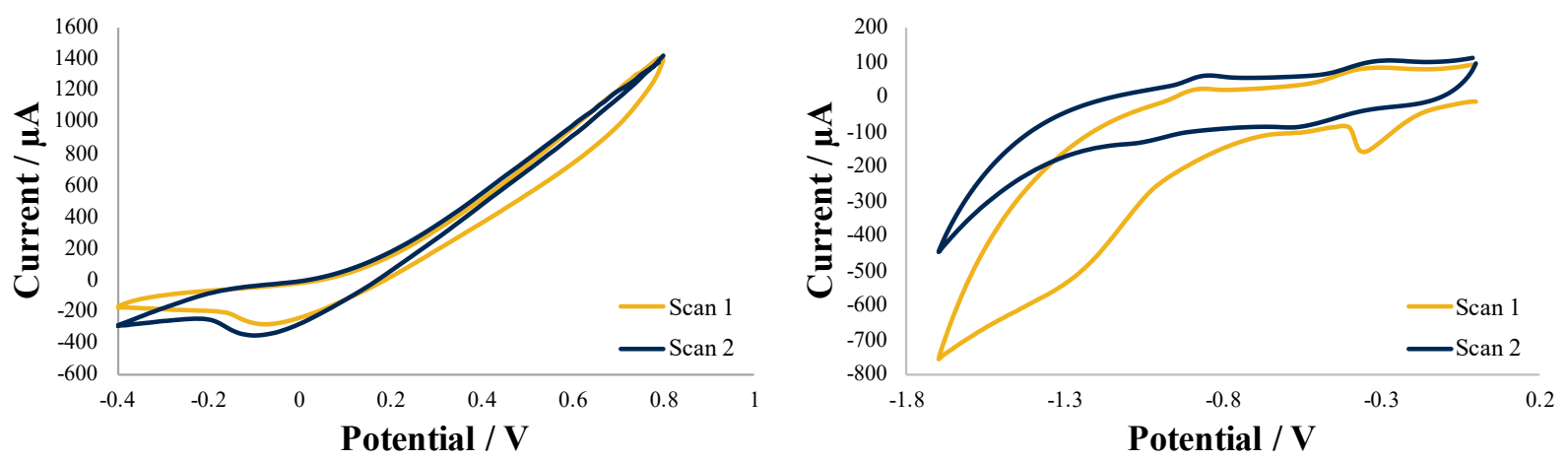

Figure 39: Attachment to the diazonium salt to the working electrode surface (left) and reduction of the nitro groups to amine groups(right). 


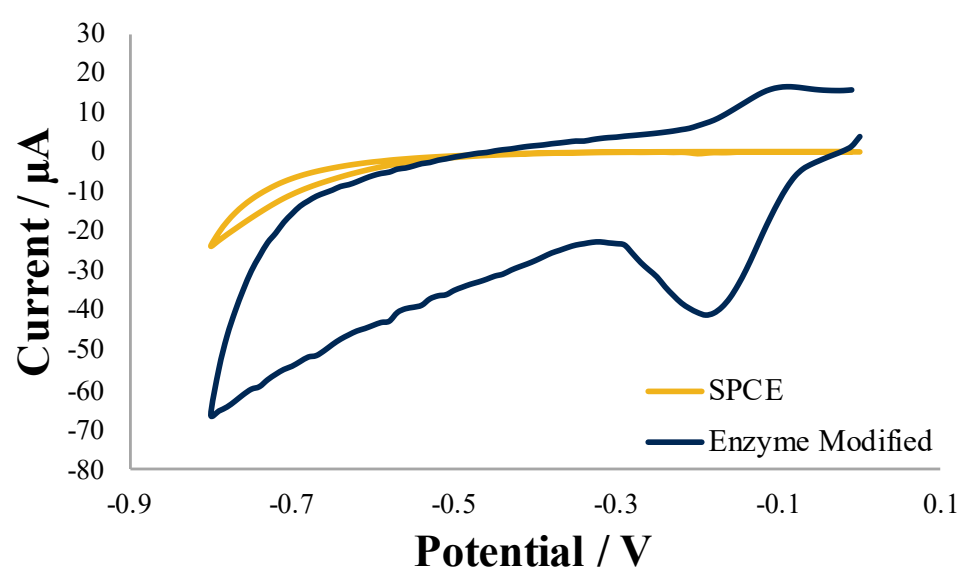

Figure 40: Cyclic voltammetry in PBS buffer demonstrating the difference between the blank SPCE and the enzyme-modified SPCE.

It was determined that there was too much noise present in measurements utilizing our instrumentation within the lab. This could have been due to the magnetic stir plate or several other instruments etc. drawing power from the building. Therefore, it was impractical to perform the analysis in a stirred solution. It was determined that analysis could be performed in a drop on the electrode surface through standard addition. Through the addition of codeine, it appeared that a current response was observed. Codeine was analyzed between $3.5 \mu \mathrm{g} / \mathrm{mL}$ and $15.5 \mu \mathrm{g} / \mathrm{mL}$ and resulted in a linear relationship with a coefficient of determination of 0.9872 . However, there were concerns regarding the shape of the resulting chronoamperogram as each step current continued a constant decrease in current (Figure 40).
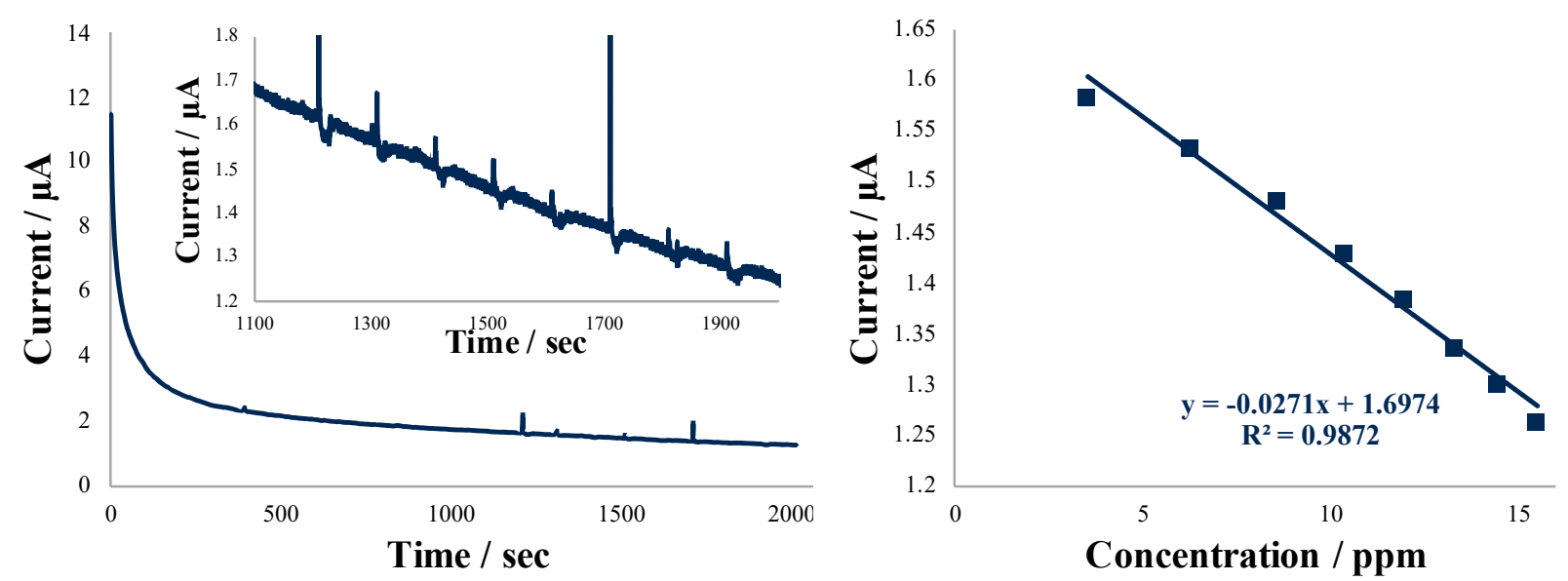

Figure 41: Chronoamperogram for the standard addition of $20 \mu \mathrm{L}$ of $30 \mu \mathrm{g} / \mathrm{mL}$ codeine to a $150 \mu \mathrm{L}$ drop of phosphate buffer (left) and resulting calibration curve (right). 


\subsection{Enzymatic Biosensor for the Detection of Fentanyl}

Due to CYP3A4 being implicated as the major CYP mediated metabolic pathway for fentanyl, an exploration into the use of CYP3A4 for the detection of fentanyl using screen-printed electrodes was performed. Immobilization of the enzyme was attempted through the modification

of a procedure described by Joseph et al., ${ }^{81}$ as well as, covalent attachment using the previously mentioned diazonium salt technique.

\subsubsection{Materials and Methods}

Fentanyl citrate was purchased from Cayman Chemical Company (Ann Arbor, MI). Ethanol 200 proof was purchased from Decon Laboratories. Glycerol was purchased from MP. Sodium hydroxide, 3-mercapto-1-propanesulfonic acid (MPS), poly-(diallyldimethylammonium chloride) 20\% wt. (PDDA), potassium chloride, monobasic sodium phosphate, and dibasic sodium phosphate were purchased from Sigma Aldrich (St. Louis, MO). Cytochrome P450 3A4 was purchased from Aviva Systems Biology at $1 \mathrm{mg} / \mathrm{mL}$.

A $1 \mathrm{mM}$ MPS solution was prepared in $18.2 \mathrm{M} \Omega$ water. A $2 \mathrm{mg} / \mathrm{mL}$ solution of PDDA was prepared in 18.2 $\mathrm{M} \Omega$ water. An electrode cleaning solution was prepared at a 60:39:1 ratio of ethanol:water: $\mathrm{NaOH}$. A $50 \mathrm{mM}$ phosphate buffer supplemented with $100 \mathrm{mM} \mathrm{KCl}(\mathrm{PBS})$ was prepared and used to prepare a solution of CYP3A4. Enzyme preparation was completed by aliquoting $1.5 \mu \mathrm{L}$ of enzyme solution with $1.5 \mu \mathrm{L} 100 \mathrm{mM}$ PBS pH 7 into a PCR tube and frozen. Before use, one tube was removed and thawed unassisted at room temperature and $4 \mu \mathrm{L}$ of $6.2 \%$ glycerol solution was added. The fentanyl solution was prepared from fentanyl citrate in PBS solution. To this end, $2 \mathrm{~mL}$ of PBS was added to a vial containing $10 \mathrm{mg}$ of fentanyl citrate for a concentration of $3125 \mu \mathrm{g} / \mathrm{mL}$ fentanyl. From this stock solution, concentrations of $0.5,1,2,4,6$, 8, 10, 50, and $100 \mu \mathrm{g} / \mathrm{mL}$ were made in PBS. A $50 \mathrm{mM}$ phosphate buffer supplemented with 100 $\mathrm{mM}$ sodium nitrate $\left(\mathrm{PBS} / \mathrm{NO}_{3}\right)$ was prepared.

Screen-printed gold electrodes (SPAuEs) were obtained from the Universidad de Burgos analytical chemistry department. SPCEs were fabricated using a DEK 248 screen-printing system 
(DEK, Weymouth, UK) with polyester screens with stencils. SPCEs contained conductive silver tracks, $\mathrm{Ag} / \mathrm{AgCl}$ pseudo-reference electrode, gold working electrode $\left(0.126 \mathrm{~cm}^{2}\right)$, and carbon counter electrode.

Prior to the immobilization of the enzyme, the gold working electrode was first cleaned by placing a drop of the 60:39:1 cleaning solution on the electrode and allowing it to sit for 30 seconds to a minute before being polished with an alumina slurry. Immobilization was achieved by placing a drop of the MPS solution on only the working electrode. This drop was allowed to evaporate or was dried under nitrogen. A drop of PDDA was then placed on the working electrode for 20 minutes before being rinsed and dried under a stream of nitrogen. Experiments were also conducted by allowing the PDDA solution to dry thoroughly on the electrode surface. Finally, a $1.5 \mu \mathrm{L}$ drop of the enzyme/glycerol solution was placed on the electrode surface for 20 minutes before being rinsed and dried under nitrogen. Again, allowing the enzyme solution to dry completely on the electrode was also investigated. The immobilization strategy is shown below in Figure 41.

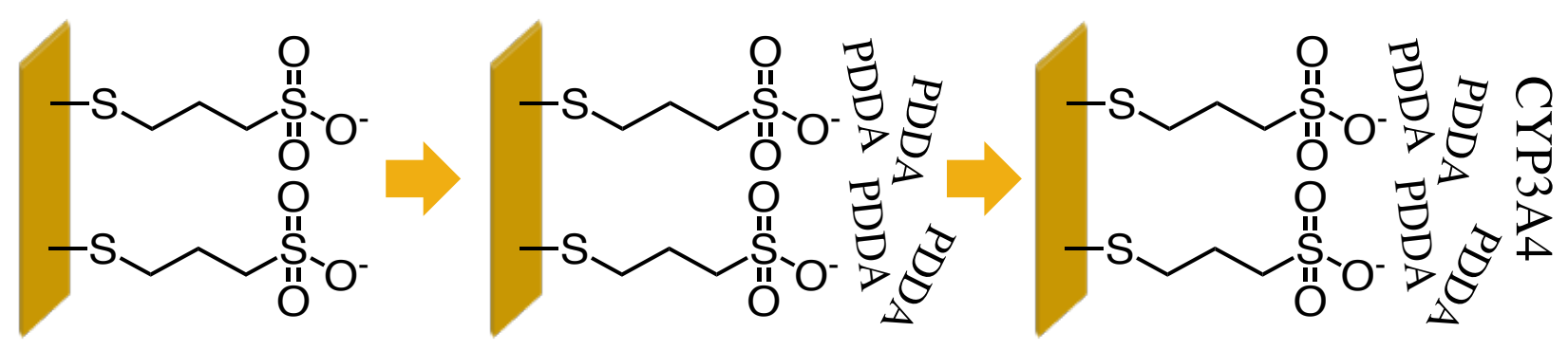

Figure 42: Immobilization strategy via self-assembled monolayer for CYP3A4 on the gold surface of the $S P_{A u} E$.

Amperometric measurement of fentanyl was performed in a $100 \mu \mathrm{L}$ drop of fentanyl and supporting electrolyte on the surface of the enzyme-modified gold electrode $\left(\mathrm{SP}_{\mathrm{Au} / \mathrm{CYP}} \mathrm{E}\right)$. To this end, a potential of $-450 \mathrm{mV}$ was applied for 60 seconds. Each concentration, including a buffer blank, was analyzed individually on the same electrode. 


\subsubsection{Instrumentation}

Electrochemical measurements were carried out using the PalmSens4 potentiostat with PSTrace software (Randhoeve, Netherlands) and the Autolab PGSTAT with NOVA software from Metrohm (Ionenstrasse, Switzerland). Measurement of solution $\mathrm{pH}$ was achieved using a Mettler Toledo FiveEasy Plus pH meter (Columbus, OH). A Millipore Direct-Q® UV water purification system (Billerica, MA) was used to obtain purified water.

\subsubsection{Results and Discussion}

Several problems encountering noise were observed when attempting to perform amperometric detection in a cell with stirring. Furthermore, the resulting chronoamperograms did not demonstrate a current response to fentanyl in an observable and reproducible way. Therefore, an amperometric analysis was carried out as a drop on the surface of the electrode. To this end, a chosen time of 60 seconds was used for the analysis of fentanyl following a similar amperometric approach as described in the literature. ${ }^{82,83}$ Analysis was carried out between fentanyl concentrations of $0.5 \mu \mathrm{g} / \mathrm{mL}$ and $100 \mu \mathrm{g} / \mathrm{mL}$. Acceptable linearity was observed with an average RSD of $17 \%$ across the concentration range (Figure 42 ).
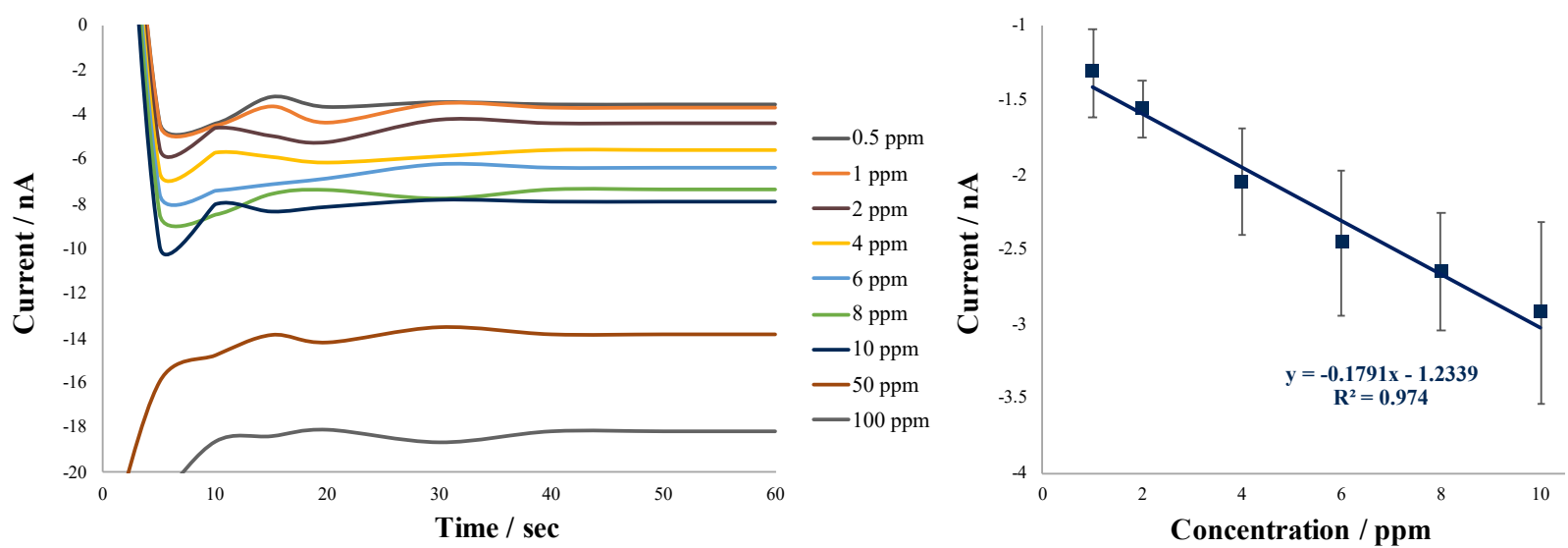

Figure 43: Constructed chronoamperograms for fentanyl detection at a SP $P_{A u}$ in a $100 \mu \mathrm{L}$ drop of $50 \mathrm{mM}$ $P B S$ supplemented with $100 \mathrm{mM} \mathrm{NaNO}$ (left) and calibration curve $(n=4)$ with $\mathrm{RSD}$ of $17 \%$. 
Attempts were made to characterize the surface of the modified electrode in order to determine that the modification procedure was achieved before the immobilization of the CYP3A4 enzyme. Scanning electron microscopy-energy dispersive X-ray spectroscopy (SEM-EDS) was used for imaging and elemental analysis of the surface. Elemental analysis of a bare $\mathrm{SP}_{\mathrm{Au}} \mathrm{E}$ demonstrated peaks due to the presence of gold, analysis of the MPS modified electrode ( $\left.\mathrm{SP}_{\mathrm{Au} / \mathrm{MPSE}}\right)$ demonstrated the presence of sodium and a shoulder in the large gold peak corresponding to sulfur. As shown in the structure of MPS and outlined in the literature,${ }^{61}$ Both sulfur and sodium are present in the structure, suggesting the presence of the MPS self-assembled monolayer on the surface of the gold electrode. Lastly, the electrode surface of the MPS/PDDA modified electrode was analyzed ( $\left.\mathrm{SP}_{\mathrm{Au} / \mathrm{MPS} / \mathrm{PDDA}} \mathrm{E}\right)$. The loss of the presence of sodium demonstrates the negative charge on the MPS layer allowing for positively charged PDDA molecule. The presence of chlorine may be attributed to the counter ion of PDDA; however, there was evidence of chlorine in the MPS modified electrode as well, but the peak was more pronounced in the PDDA layer. Figure 43 demonstrates the acquired SEM-EDS spectra.

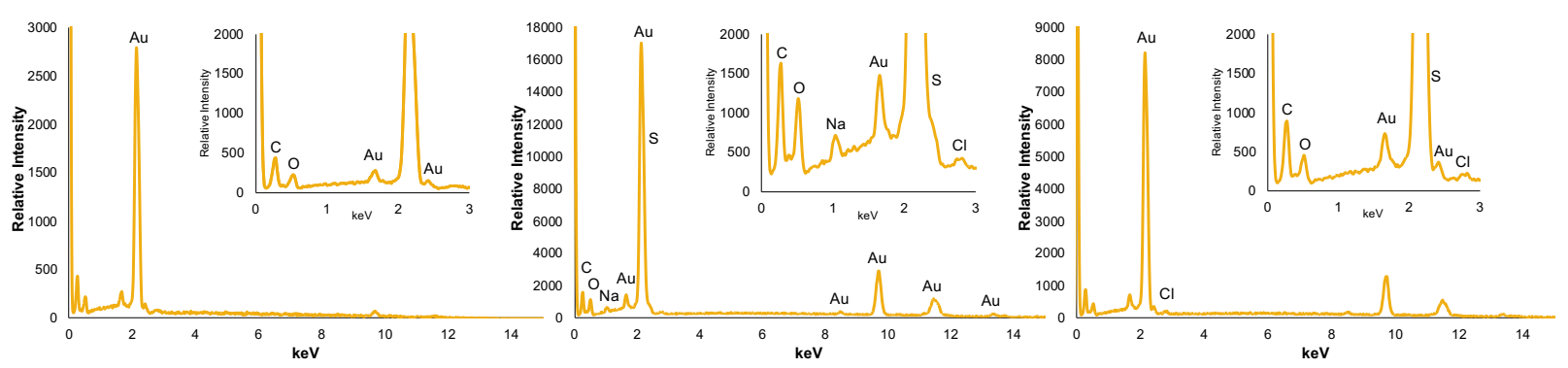

Figure 44: SEM-EDS spectra for a bare $S P_{A u} E$ (left), MPS modified $S P_{A u / M P S} E$ (middle), and PDDA modified $S P_{A u / M P S / P D D A} E$ (right).

The use of both SEM and atomic force microscopy (AFM) was used to attempt to visualize and characterize the electrode surface as well. Little to no difference was observed between the modification steps of the electrode with SEM of AFM. The gold surface of the $\mathrm{SP}_{\mathrm{Au}} \mathrm{E}$ was observed to be tightly packed spherical particles at high magnification and at low magnification, surface imperfections were visible on the working electrode (Figure 44). AFM was not useful in discerning surface differences due to the modification of the electrode. AFM images plotting height can be seen in Figure 45. 
Part V: Other Electrochemical Methods
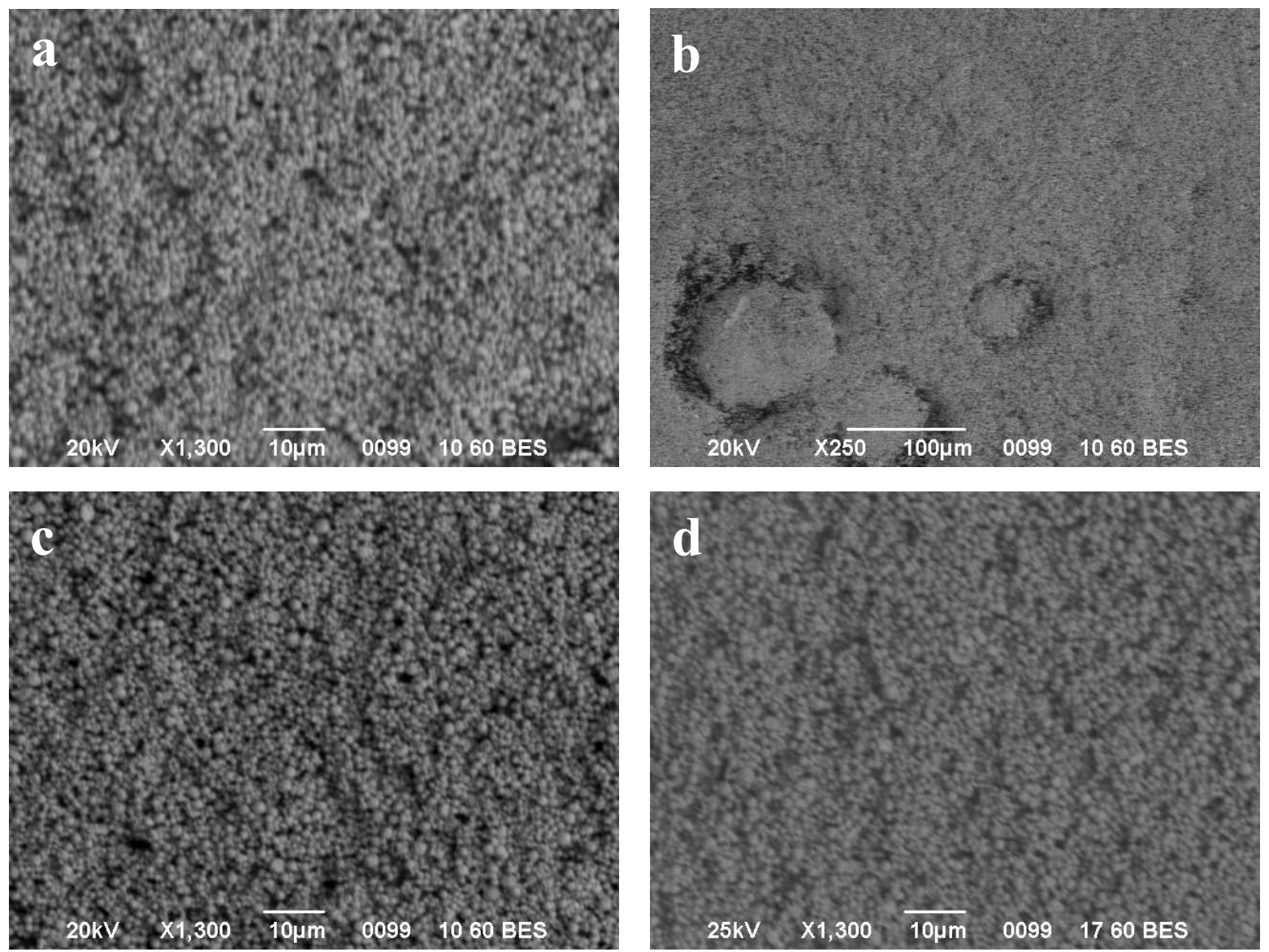

Figure 45: SEM images for the blank $S P_{A u} E$ ( $a$ and $b$ ), the MPS modified electrode (c), and the MPS/PDDA modified electrode (d).
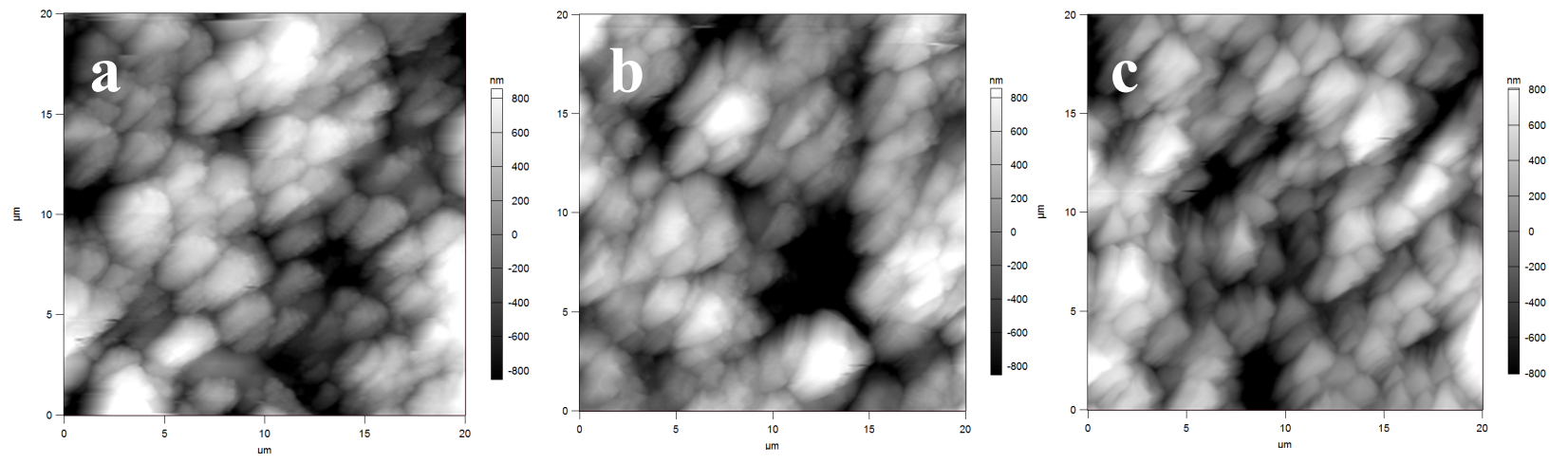

Figure 46: AFM images plotting the height of the blank $S P_{A u} E(a), M P S$ modified electrode (b), and MPS/PDDA modified electrode (c). 


\subsubsection{Conclusion}

Although it was initially observed to demonstrate a response to fentanyl, this method was not appropriate due to the determination that the signal was from chloride in solution. Upon further investigation of the amperometric sensor, it was discovered that the current response was likely due to chloride ion in the PBS buffer and not to fentanyl itself. This lack of signal for fentanyl could be due to loss of the enzyme from the electrode into the solution, loss of conformation of the enzyme resulting in lost activity, too small an enzyme concentration, or inappropriate potential applied. Later experiments performed using square-wave voltammetry demonstrated the oxidation of fentanyl at a positive potential. However, the potential applied to the enzyme-modified electrode was required for the electron transfer with the enzyme and was not directly involved in the electron transfer with fentanyl. The potential applied was similar to what is found in the literature for CYP 3A4. ${ }^{84-86}$ Failure of this method prompted exploration into other methods for the detection of fentanyl using screen-printed carbon electrodes. Although the use of only PBS supplemented with nitrate was attempted, signal response to fentanyl could not be confirmed and this method as abandoned for detection of fentanyl.

\subsection{Detection of the Synthetic Cannabinoid PB-22}

The detection of PB-22 was investigated to explore electrochemical methods for the detection of synthetic cannabinoids. The redox process of PB-22 was explored using a conventional platinum electrode following a modified procedure by Dronova et al. ${ }^{87}$

\subsubsection{Materials and Methods}

PB-22 was purchased as neat solid from Cayman Chemical (Ann Arbor, MI). Conventional platinum working and auxiliary electrodes and a $\mathrm{Ag} / \mathrm{AgCl}$ reference electrode were purchased from Metrohm (Ionenstrasse, Switzerland). Tetrabutylammonium perchlorate (TBAP) was purchased from Acros Organics (Fair Lawn, NJ). Acetonitrile (Optima $\left.{ }^{\circledR}\right)$, Methanol (Optima ${ }^{\circledR}$ ), and sulfuric acid (Trace Metal Grade) were purchased from Fisher Scientific (Fair Lawn, NJ). 
Supporting electrolyte was prepared as $10 \mathrm{mM}$ TBAP in acetonitrile. PB-22 was prepared by dissolving $5 \mathrm{mg}$ of neat solid in $10 \mathrm{~mL}$ of methanol for a final concentration of $500 \mu \mathrm{g} / \mathrm{mL}$. A solution of $0.5 \mathrm{M}$ sulfuric acid was prepared from concentrated. The platinum working electrode was polished using alumina powder and water in figure-eights in both directions for 30-60 seconds. Following a brief rinsing of the electrode with water, it was electrochemically cleaned in $0.5 \mathrm{M}$ sulfuric acid using cyclic voltammetry between $-0.21 \mathrm{~V}$ to $1.5 \mathrm{~V}$ vs. $\mathrm{Ag} / \mathrm{AgCl}$ for 20 scans at a rate of $0.2 \mathrm{~V} / \mathrm{sec}$.

Analysis of PB-22 was conducted in the electrochemical cell at concentrations between $0.208 \mu \mathrm{g} / \mathrm{mL}$ and $6.7 \mu \mathrm{g} / \mathrm{mL}$. Differential pulse voltammetry was carried out from $1.8 \mathrm{~V}$. to $0.7 \mathrm{~V}$ with a step of $-0.008 \mathrm{~V}$, modulation amplitude of $0.14 \mathrm{~V}$, modulation time of 0.05 seconds, and an interval time of 1 second. The working electrode surface was polished and electrochemically cleaned between all measurements.

\subsubsection{Instrumentation}

Electrochemical measurements were carried out using the Autolab PGSTAT with NOVA software from Metrohm (Ionenstrasse, Switzerland). A Millipore Direct-Q ${ }^{\circledR}$ UV water purification system (Billerica, MA) was used to obtain purified water.

\subsubsection{Results and Discussion}

The importance of the polishing and cleaning procedure was demonstrated through the analysis of replicate concentrations of PB-22. Repeatability was excellent for analysis of samples following a thorough cleaning of the working electrode. However, when attempting to analyze samples without the cleaning step, the peak heights were not reproducible. The cyclic voltammogram for the cleaning procedure can be seen in Figure 46. 


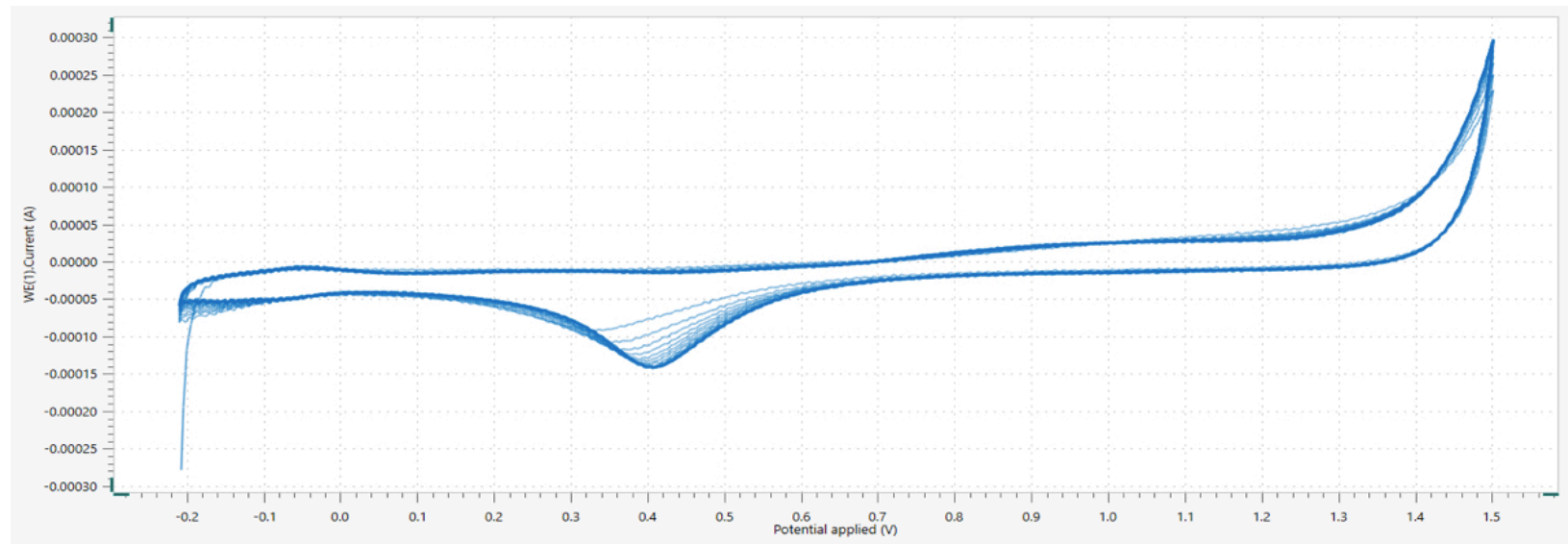

Figure 47: Cyclic voltammogram in $0.5 \mathrm{M}$ sulfuric acid for electrochemical cleaning the platinum working electrode.

Electrochemical detection of PB-22 was achieved utilizing a platinum working electrode with platinum auxiliary electrode and $\mathrm{Ag} / \mathrm{AgCl}$ reference electrode. Analysis concentrations were achieved through dilution of the previous samples. The differential pulse method utilized yielded an observable reduction peak at the lowest concentration of approximately 208 parts-per-billion. The average peak height at the lowest concentration was approximately $-5.5 \times 10^{-7} \mathrm{~A}$. The noise was estimated to be between 100-200 nA making the current response between 3 and 5 times the noise level. Standard deviation of peak height between replicates was between $\sim-3.4 \times 10^{-7} \mathrm{~A}$ and $-1.1 \times 10^{-6} \mathrm{~A}$ for the smallest and largest concentrations, respectively. A standard curve was generated that included the largest concentration and had an $\mathrm{R}^{2}$ value of 0.9814 . By removing the highest concentration, the new $\mathrm{R}^{2}$ value of 0.99955 was achieved (Figure 47).
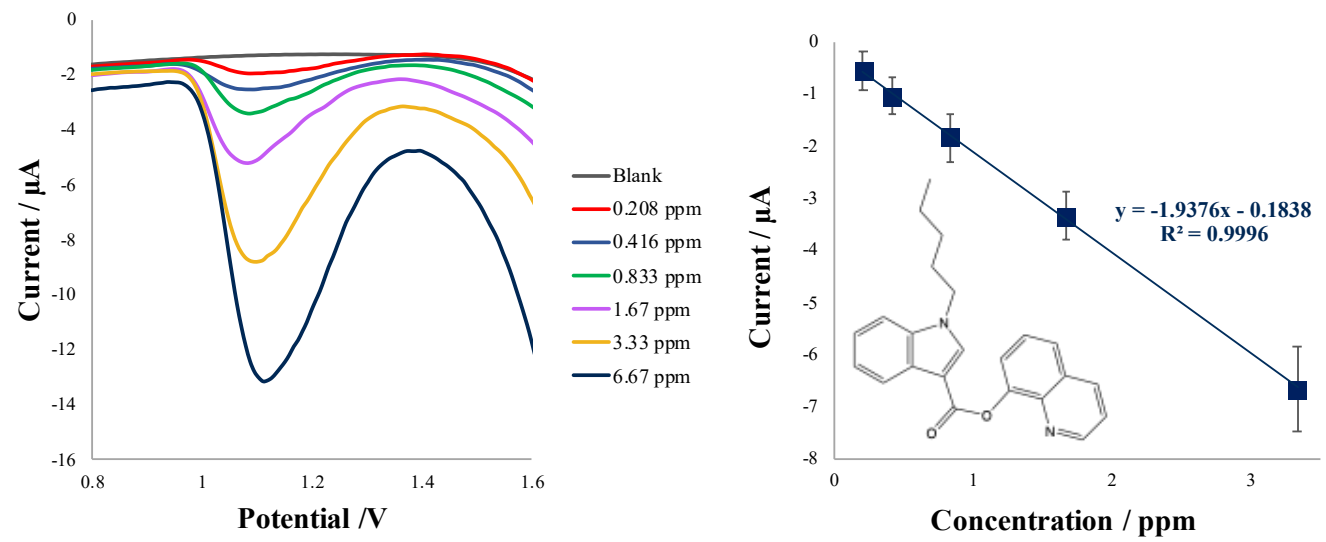

Figure 48: Differential pulse voltammograms for analysis of PB-22 in an electrochemical cell (left) and the resulting calibration curve for 3 replicates (right). 
Part V: Other Electrochemical Methods

\subsubsection{Conclusions}

Detection of the synthetic cannabinoid PB-22 was demonstrated to be achievable utilizing the differential pulse method described above. Excellent linearity was achieved for the method between $\sim 0.200 \mu \mathrm{g} / \mathrm{mL}$ and $3.33 \mu \mathrm{g} / \mathrm{mL}$. This method differed from the majority of electrochemical methods due to the analysis of the analyte in an organic medium rather than an aqueous medium. Future work will entail the detection of other synthetic cannabinoids using both conventional electrodes and organic-resistant screen-printed electrodes. 


\section{References}

(1) About the U.S. Opioid Epidemic. United States Department of Health and Human Services. https://www.hhs.gov/opioids/about-the-epidemic/index.html\#us-epidemic.

(2) Public Health Emergency Declarations. United States Department of Health and Human Services. https://www.phe.gov/emergency/news/healthactions/phe/Pages/default.aspx.

(3) Opioid Overdose. Centers for Disease Control and Prevention. https://www.cdc.gov/drugoverdose/index.html.

(4) Leventhal, R. West Virginia calls in the National Guard to tackle opioid crisis http://www.foxnews.com/health/2018/02/09/west-virginia-calls-in-national-guard-totackle-opioid-crisis.html.

(5) Opioid Overdose: Drug Overdose Death Data. Centers for Disease Control and Prevention. https://www.cdc.gov/drugoverdose/data/statedeaths.html.

(6) Behavioral Health Barometer: West Virginia, Volume 4: Indicators as measured through the 2015 National Survey on Drug Use and Health, the National Survey of Substance Abuse Treatment Services, and the Uniform Reporting System; United States Department of Health and Human Services, 2017; Vol. 4.

(7) Zawilska, J. B. Front. Psychiatry 2017, 8 (JUN), 1-14.

(8) Menendez, M. J.; Weedn, V.W. Collaboration in the Fight Against Fentanyl. Organized Crime Drug Enforcement Task Forces and George Washington University.

(9) Head, J. M. Synthetic Drug Threats in the United States: 2017 Update. Special Testing and Research Laboratory, Drug Enforcement Administration.

(10) Synthetic Opioid Overdose Data. Centers for Disease Control and Prevention. https://www.cdc.gov/drugoverdose/data/fentanyl.html.

(11) Vardanyan, R. S.; Hruby, V. Future Med Chem. 2014, 6 (4), 385-412.

(12) Smith, J. P.; Randviir, E. P.; Banks, C. E. Forensic Science: A Multidisciplinary Approach. 2016,

(13) Lowe, E. R.; Banks, C. E.; Compton, R. G. Anal. Bioanal. Chem. 2005, 383 (3), 523-531.

(14) Merli, D.; Zamboni, D.; Protti, S.; Pesavento, M.; Profumo, A. Talanta 2014, 130, 456461.

(15) Jiang, B.; Wang, M.; Chen, Y.; Xie, J.; Xiang, Y. Biosens. Bioelectron. 2012, 32 (1), 305308. 
(16) de Oliveira, L. S.; Poles, A. P. dos S.; Balbino, M. A.; de Menezes, M. M. T.; de Andrade, J. F.; Dockal, E. R.; Tristão, H. M.; de Oliveira, M. F. Sensors (Switzerland) 2013, 13 (6), 7668-7679.

(17) Asturias-Arribas, L.; Alonso-Lomillo, M. A.; Domínguez-Renedo, O.; Arcos-Martínez, M. J. Talanta 2013, 105, 131-134.

(18) Smith, J. P.; Metters, J. P.; Irving, C.; Sutcliffe, O. B.; Banks, C. E. Analyst 2014, 139 (2), 389-400.

(19) Asturias-Arribas, L.; Asunción Alonso-Lomillo, M.; Domínguez-Renedo, O.; Julia ArcosMartínez, M. Talanta 2014, 129, 315-319.

(20) Garrido, J. M. P. J.; Delerue-Matos, C.; Borges, F.; Macedo, T. R. A.; Oliveira-Brett, A. M. Electroanalysis 2004, 16 (18), 1497-1502.

(21) Narcotics (Opioids). Drug Enforcement Administration. https://www.dea.gov/taxonomy/term/331.

(22) Smith, H. S. Mayo Clin. Proc. 2009, 84 (7), 613-624.

(23) Opioid painkillers: How they work and why they can be risky. National Safety Council, 2014.

(24) Feierman, D. E.; Lasker, J. M. Drug Metab. Dispos. 1996, 24 (9), 932-939.

(25) Tateishi, T.; Krivoruk, Y.; Ueng, Y.-F.; Wood, A. J. J.; Guengerich, F. P.; Wood, M. Anesth. Analg. 1996, 82 (1), 167-172.

(26) Baselt, R. C.; Cravey, R. H. Disposition of Toxic Drugs and Chemicals in Man, 3rd ed.; Year Book Medical Publishers, Inc., 1989.

(27) Bista, S. R.; Lobb, M.; Haywood, A.; Hardy, J.; Tapuni, A.; Norris, R. J. Chromatogr. B 2014, 960, 27-33.

(28) Sisco, E.; Verkouteren, J.; Staymates, J.; Lawrence, J. Forensic Chem. 2017, 4, 108-115.

(29) Laboratory guide for fentanyl identification, naming, and metabolism. Caymen Chemical Company, 2018.

(30) Dai, H.; Xu, H.; Wu, X.; Chi, Y.; Chen, G. Anal. Chim. Acta 2009, 647, 60-65.

(31) Elbardisy, H. M.; Foster, C. W.; Cumba, L.; Antonides, L. H.; Gilbert, N.; Schofield, C. J.; Belal, T. S.; Talaat, W.; Sutcliffe, O. B.; Daabees, H. G.; Banks, C. E. Anal. Methods 2019, 11 (8), 1053-1063.

(32) Guo, H.; Hu, N.; Lin, S. Talanta 1994, 41 (11), 1929-1932. 
(33) Goodchild, S. A.; Hubble, L. J.; Mishra, R. K.; Li, Z.; Goud, K. Y.; Barfidokht, A.; Shah, R.; Bagot, K. S.; McIntosh, A. J. S.; Wang, J. Anal. Chem. 2019, 91 (5), 3747-3753.

(34) Barfidokht, A.; Mishra, R. K.; Seenivasan, R.; Liu, S.; Hubble, L. J.; Wang, J.; Hall, D. A. Sensors Actuators, B Chem. 2019.

(35) Ahmar, H.; Fakhari, A. R.; Tabani, H.; Shahsavani, A. Electrochim. Acta 2013, 96, 117123.

(36) Strobel, H. A.; Heineman, W. R. Chemical Instrumentation: A Systematic Approach, 3rd ed.; John Wiley \& Sons, Inc., 1989.

(37) Farghaly, O. A.; Abdel Hameed, R. S.; Abu-Nawwas, A.-A. H. Int. J. Electrochem. Sci. 2014, 9, 3287-3318.

(38) Cunha-Silva, H.; Arcos-Martinez, M. J. Sensors Actuators, B Chem. 2019, 282, 603-608.

(39) Balbino, M. A.; de Oliveira, L. S.; Eleotério, I. C.; Oiye, E. N.; Ribeiro, M. F. M.; McCord, B. R.; Ipolito, A. J.; de Oliveira, M. F. J. Forensic Sci. 2016, 61 (4), 1067-1073.

(40) Townsend, S.; Fanning, L.; O’Kennedy, R. Anal. Lett. 2008, 41, 925-948.

(41) Putzbach, W.; Ronkainen, N. J. Sensors 2013, 13, 4811-4840.

(42) da Silva, E. T. S. G.; Souto, D. E. P.; Barragan, J. T. C.; de F. Giarola, J.; de Moraes, A. C. M.; Kubota, L. T. ChemElectroChem 2017, 4 (4), 778-794.

(43) Zhu, C.; Yang, G.; Li, H.; Du, D.; Lin, Y. Anal. Chem. 2015, 87 (1), 230-249.

(44) Ronkainen, N. J.; Halsall, H. B.; Heineman, W. R. Chem. Soc. Rev. 2010, 39, 1747-1763.

(45) Schneider, E.; Clark, D. S. Biosens. Bioelectron. 2013, 39 (1), 1-13.

(46) Li, M.; Li, R.; Li, C. M.; Wu, N. Front. Biosci. 2011, S3, 1308-1331.

(47) Yang, M.; Kabulski, J. L.; Wollenberg, L.; Chen, X.; Subramanian, M.; Tracy, T. S.; Lederman, D.; Gannett, P. M.; Wu, N. Drug Metab. Dispos. 2009, 37 (4), 892-899.

(48) Sweeney, B. P.; Bromilow, J. Anaesthesia 2006, 61, 159-177.

(49) Poulos, T. L. Chem. Rev. 2014, 114, 3919-3962.

(50) Denisov, I. G.; Makris, T. M.; Sligar, S. G.; Schlichting, I. Chem. Rev. 2005, 105, 22532277.

(51) Gilardi, G.; Nardo, G. Di. Rend. Lincei 2017, 28 (s1), S159-S167.

(52) CYP2D6 gene:Cytochrome P450 family 2 subfamily D member 6. National Institute of Health: United States National Library of Medicine. https:/ghr.nlm.nih.gov/gene/CYP2D6\#conditions (accessed Mar 19, 2018). 
(53) UniProtKB-P10635 (CP2D6_HUMAN) https://www.uniprot.org/uniprot/P10635 (accessed Mar 19, 2018).

(54) Rowland, P.; Blaney, F. E.; Smyth, M. G.; Jones, J. J.; Leydon, V. R.; Oxbrow, A. K.; Lewis, C. J.; Tennant, M. G.; Modi, S.; Eggleston, D. S.; Chenery, R. J.; Bridges, A. M.; Cytochrome, P.; Structure, C. J. Biol. Chem. 2006, 281 (11), 7614-7622.

(55) Gundert-Remy, U.; Bernauer, U.; Blömeke, B.; Döring, B.; Fabian, E.; Goebel, C.; Hessel, S.; Jäckh, C.; Lampen, A.; Oesch, F.; Petzinger, E.; Völkel, W.; Roos, P. H. Drug Metab. Rev. 2914, 46 (3), 291-324.

(56) Samer, C. F.; Lorenzini, K. I.; Rollason, V.; Daali, Y.; Desmeules, J. A. Mol. Diagnosis Ther. 2013, 17 (3), 165-184.

(57) Yano, J. K.; Wester, M. R.; Schoch, G. A.; Griffin, K. J.; Stout, C. D.; Johnson, E. F. J. Biol. Chem. 2004, 279 (37), 38091-38094.

(58) Wang, J. Analyst 2005, 130 (4), 421-426.

(59) Sanghavi, B. J.; Wolfbeis, O. S.; Hirsch, T.; Swami, N. S. Microchim. Acta 2015, 182 (12), $1-41$.

(60) Haddad, A.; Comanescu, M. A.; Green, O.; Kubic, T. A.; Lombardi, J. R. Anal. Chem. 2018, 90 (21), 12678-12685.

(61) Cheng, H. W.; Thiagarajan, S.; Chen, S. M. Int. J. Electrochem. Sci. 2011, 6 (9), 41504163.

(62) Barquero-Quirós, M.; Domínguez-Renedo, O.; Alonso-Lomillo, M. A.; Arcos-Martínez, M. J. Sensors (Switzerland) 2014, 14 (5), 8203-8216.

(63) Barquero-Quirós, M.; Arcos-Martínez, M. J. Sensors (Switzerland) 2016, 16 (10), 1-19.

(64) Renedo, O. D.; Alonso-Lomillo, M. A.; Martínez, M. J. A. Talanta 2007, 73 (2), 202-219.

(65) ASB Standard 036, First Edition Standard Practices for Method Validation in Forensic Toxicology; 2017.

(66) Behpour, M.; Valipour, A.; Keshavarz, M. Mater. Sci. Eng. C 2014, 42, 500-505.

(67) Shahrokhian, S.; Kamalzadeh, Z.; Saberi, R. S. Electroanalysis 2011, 23 (12), 2925-2934.

(68) Ghorbani-Bidkorbeh, F.; Shahrokhian, S.; Mohammadi, A.; Dinarvand, R. J. Electroanal. Chem. 2010, 638 (2), 212-217.

(69) Majidi, M. R.; Ghaderi, S.; Asadpour-Zeynali, K.; Dastangoo, H. Mater. Sci. Eng. C 2015, $57,257-264$. 
(70) Marinetti, L. J.; Ehlers, B. J. J. Anal. Toxicol. 2014, 38 (8), 592-598.

(71) Masui, M. J. Chem. Soc. B 1968, 01, 973-976.

(72) Frisell, W. R.; Chung, C. W.; Mackenzie, C. G. J. Biol. Chem. 1959, 234 (5), 1297-1301.

(73) Smith, J. R. L. J. Chem. Soc., Perkin Trans. 2 1977, 01 (13), 1732-1736.

(74) Hegde, R. N.; Hosamani, R. R.; Nandibewoor, S. T. Colloids Surfaces B Biointerfaces 2009, 72 (2), 259-265.

(75) Guidelines for Testing Drugs under International Control in Hair, Sweat and Oral Fluid: Manual for use by national drug analysis laboratories; United Nations Office on Drugs and Crime, Vienna, 2014.

(76) Griswold, M. K.; Chai, P. R.; Krotulski, A. J.; Friscia, M.; Chapman, B. P.; Varma, N.; Boyer, E. W.; Logan, B. K.; Babu, K. M. J. Med. Toxicol. 2017, 13 (4), 287-292.

(77) Heltsley, R.; DePriest, A.; Blank, D. L.; Crouch, D.J.; Robert, T.; Marshall, L.; Medors, V.M.; Caplan, Y.H.; Cone, E.J. J. Anal. Toxicol. 2011, 36 (January), 75-80.

(78) Peters, F. T. In Applications of LC-MS in Toxicology; Polettini, A., Ed.; Pharmaceutical Press: London, 2006; pp 71-90.

(79) Eurachem Guide: The Fitness for Purpose of Analytical Methods - A laboratory guide to method validation and related topics, 2nd ed.; Magnusson, B., Örnemark, U., Eds.; 2014.

(80) Matuszewski, B. K.; Constanzer, M. L.; Chavez-Eng, C. M. Anal. Chem. 2003, 75 (13), 3019-3030.

(81) Joseph, S.; Rusling, J. F.; Lvov, Y. M.; Friedberg, T.; Fuhr, U. Biochem. Pharmacol. 2003, 65 (11), 1817-1826.

(82) Nicholas, P.; Pittson, R.; Hart, J. P. Food Chem. 2018, 241, 122-126.

(83) Jia, W.; Bandodkar, A. J.; Valdés-Ramírez, G.; Windmiller, J. R.; Yang, Z.; Ramírez, J.; Chan, G.; Wang, J. Anal. Chem. 2013, 85 (14), 6553-6560.

(84) Xue, Q.; Kato, D.; Kamata, T.; Guo, Q.; You, T.; Niwa, O. Analyst 2013, 138 (21), 64636468.

(85) Müller, M.; Agarwal, N.; Kim, J. Biosensors 2016, 6 (3), 1-15.

(86) Ndangili, P. M.; Jijana, A. M.; Baker, P. G. L.; Iwuoha, E. I. J. Electroanal. Chem. 2011, $653(1-2), 67-74$.

(87) Dronova, M.; Smolianitski, E.; Lev, O. Anal. Chem. 2016, 88 (8), 4487-4494. 


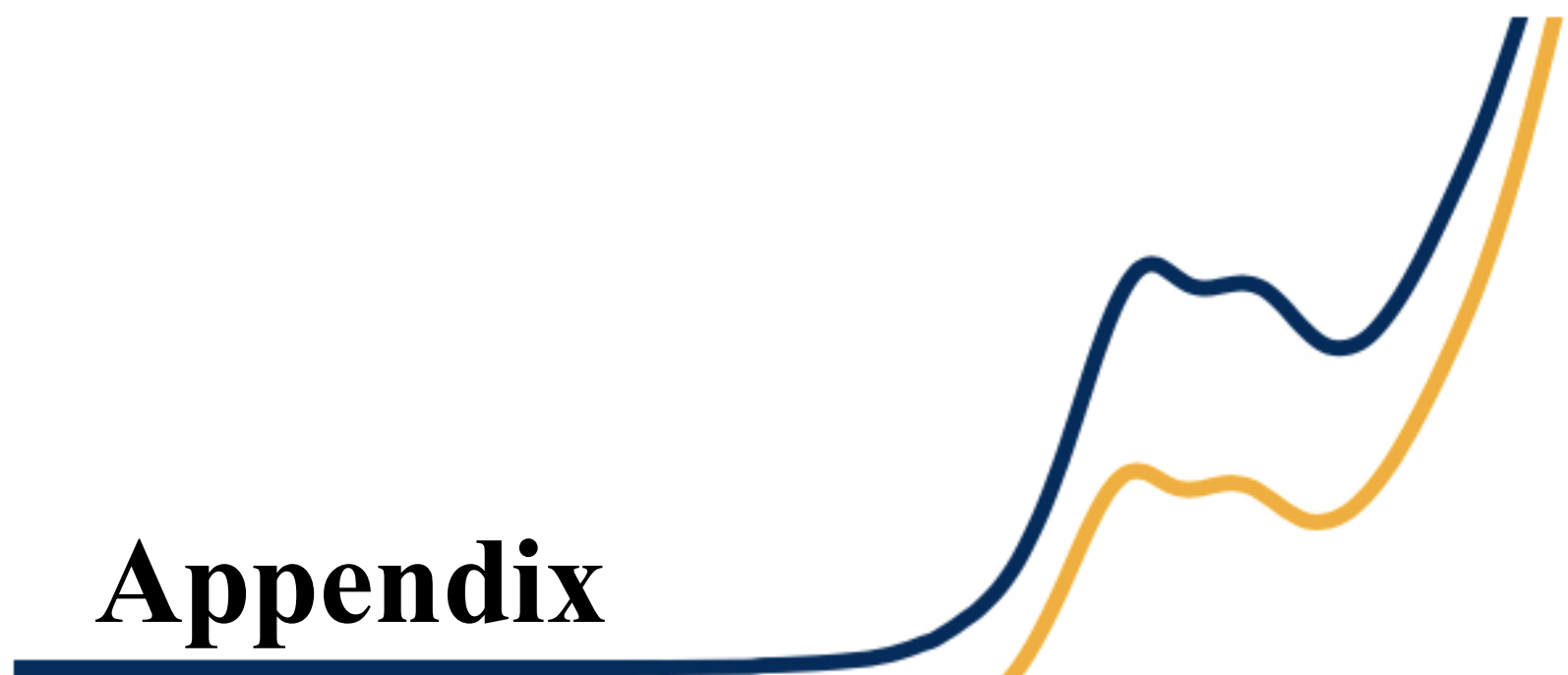


Table A1: Structures of Target Analytes

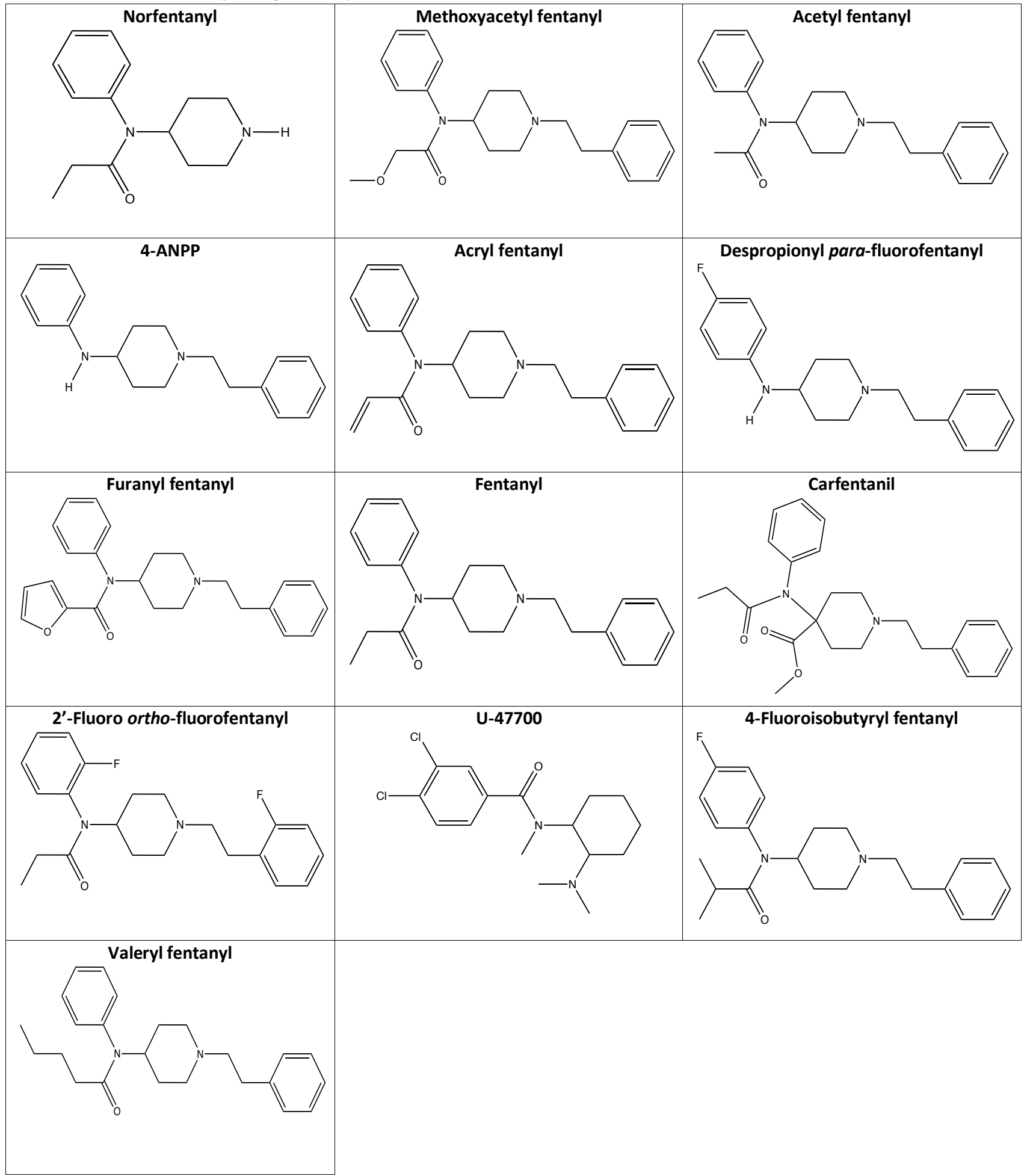




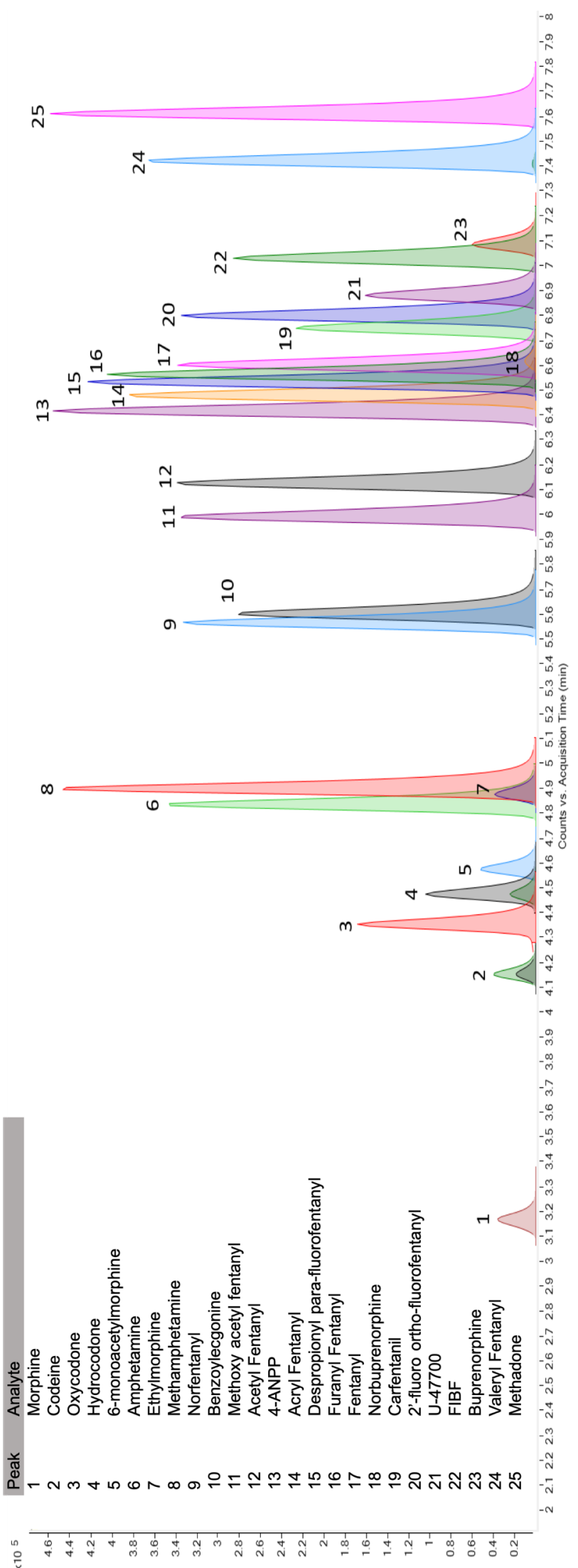

Figure A1: Chromatographic separation of analytes of interest and other illicit drugs. 


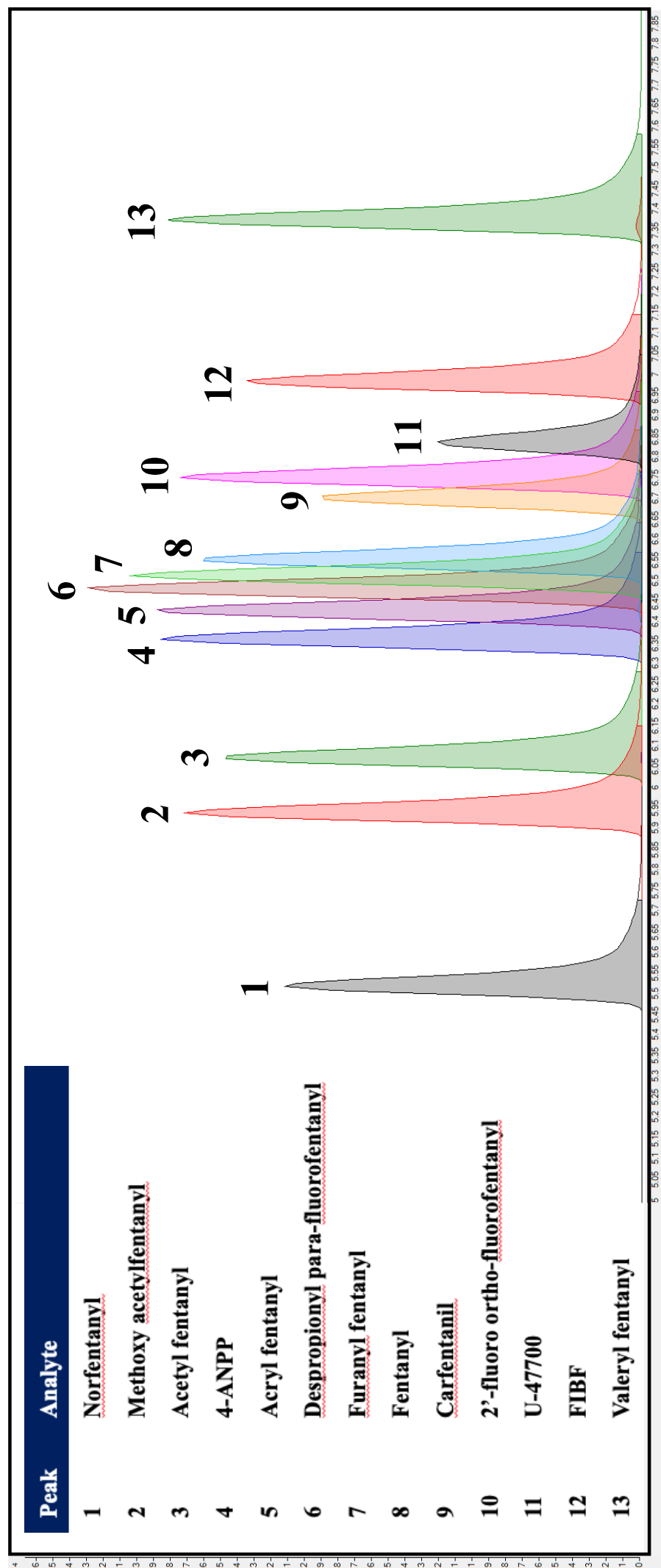

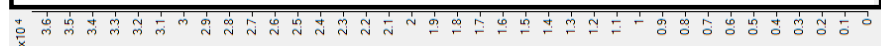

Figure A2: Chromatographic separation of analytes of interest. 


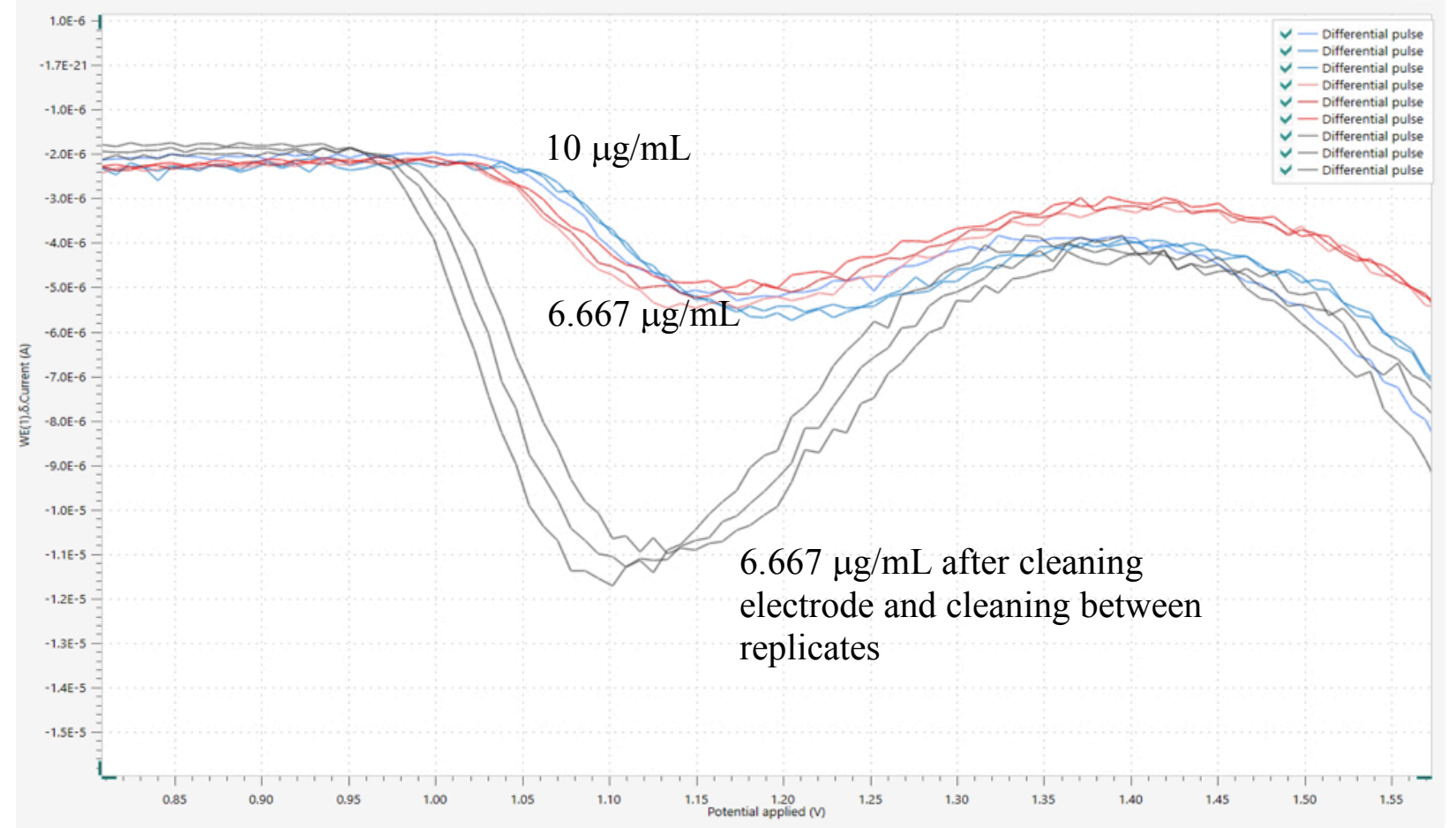

Figure A3: Differential pulse voltammograms for PB-22 demonstrating the need to clean the electrode surface.

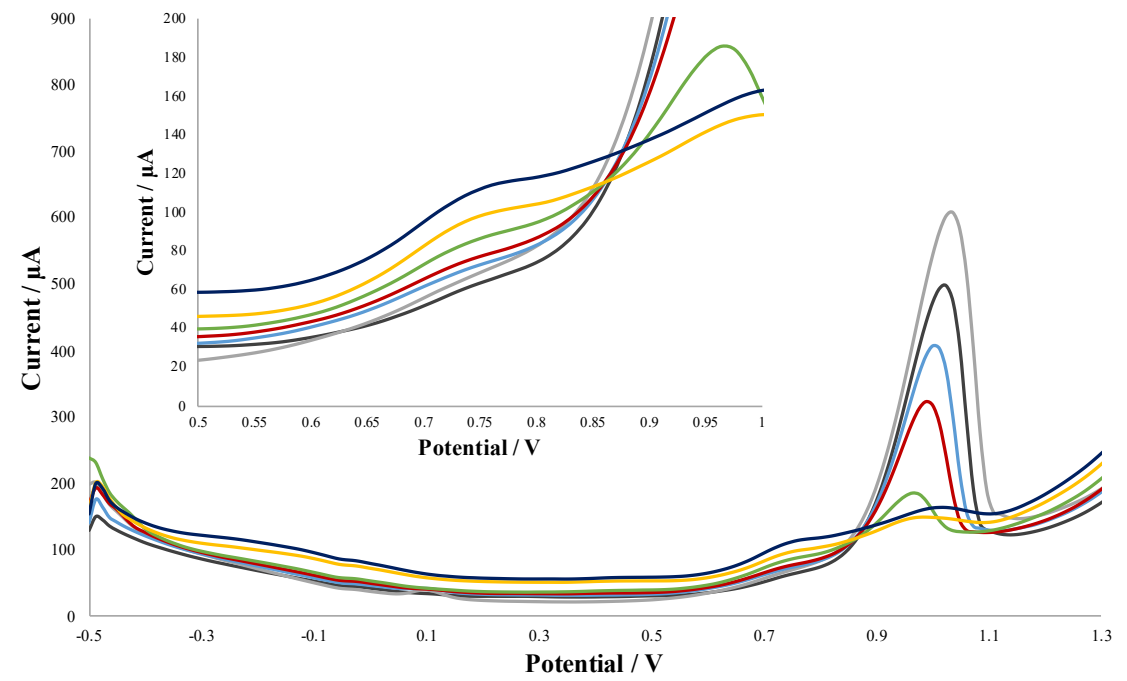

Figure A4: Adsorptive stripping square-wave voltammograms for the detection of fentanyl at gold nanoparticle modified SPCE in Tris- $\mathrm{HCl}$. 


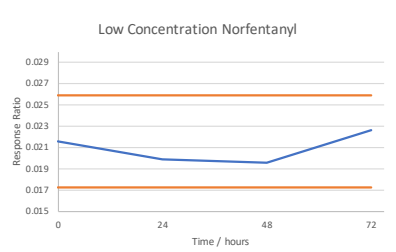

High Concentration Norfentanyl

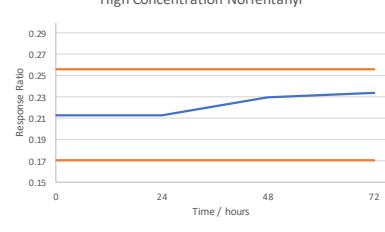

Low Concentration Acryl fentanyl

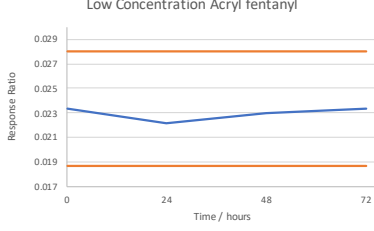

High Concentration Acryl fentany

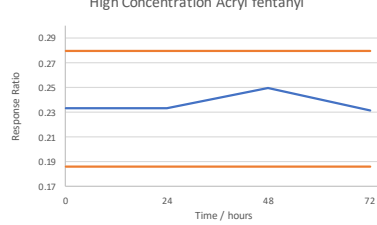

Low Concentration Carfentanil
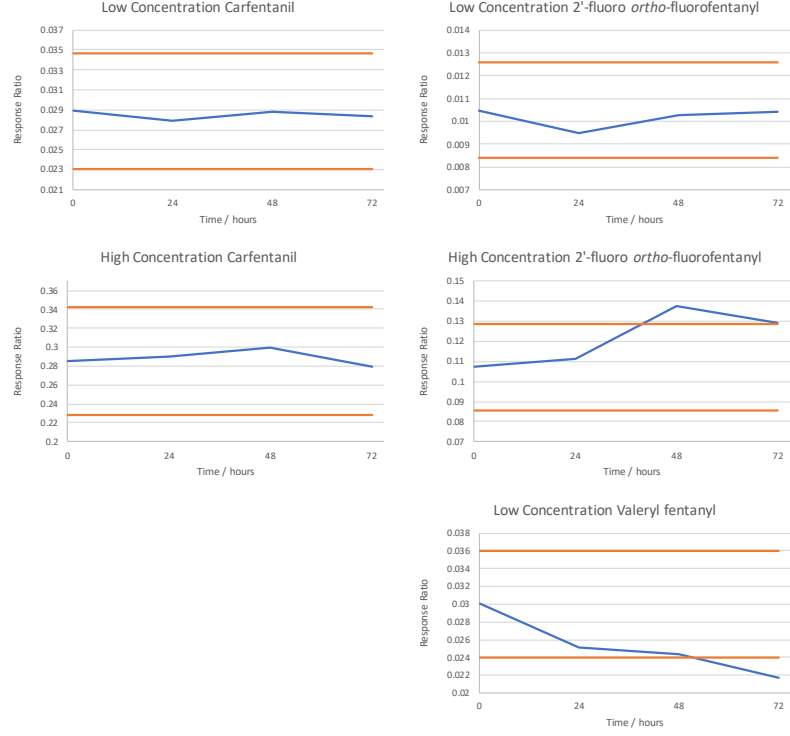
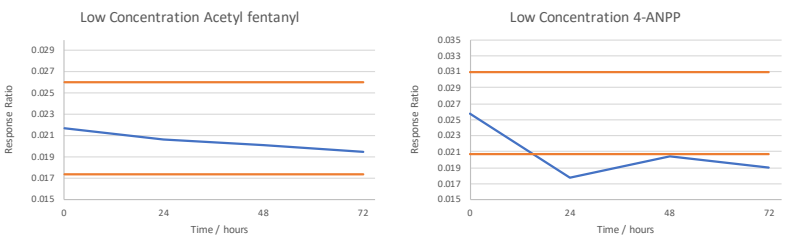

High Concentration Acetyl fentanyl

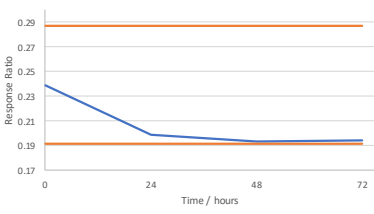

High Concentration 4-ANP

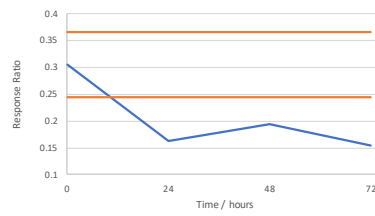

Low Concentration Fentanyl
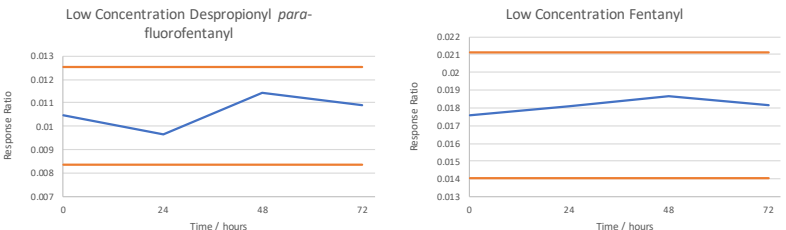

High Concentration Fentanyl
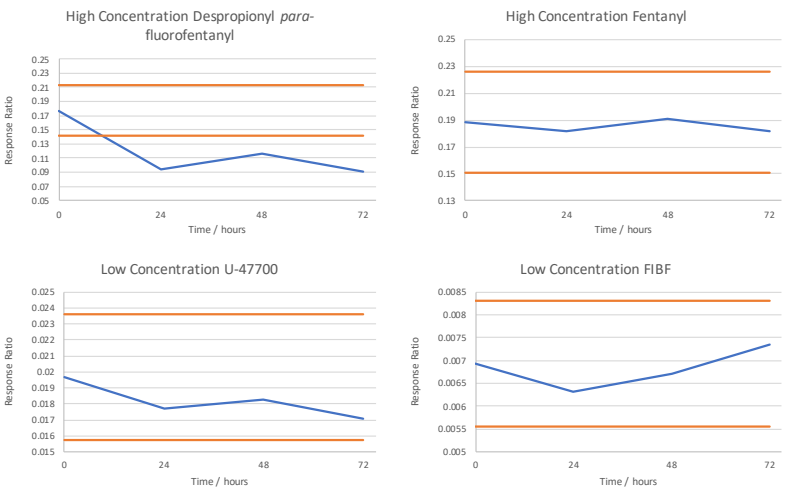

High Concentration U-47700
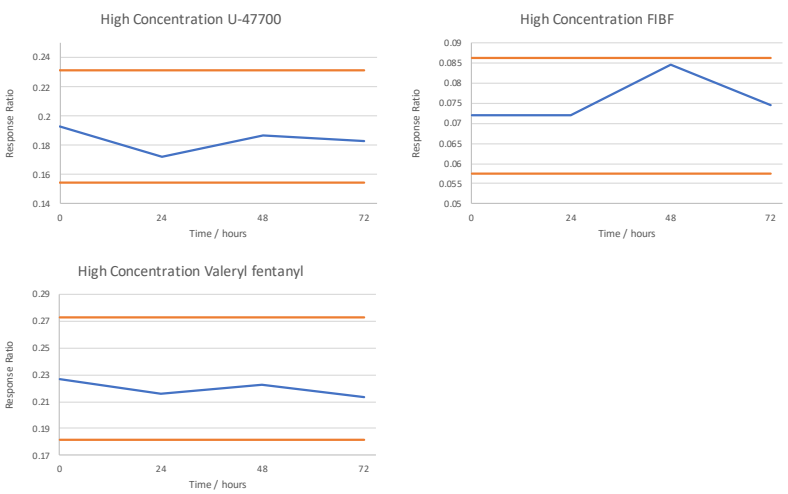

Figure A5: Plots demonstrating response ratio versus time, representing the stability of analytes at both the low and high concentrations over three freeze/thaw cycles. 
Low Concentration Norfentanyl

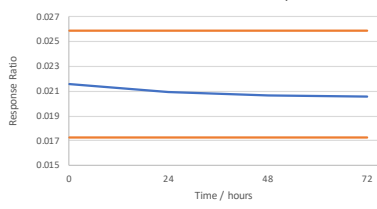

High Concentration Norfentanyl

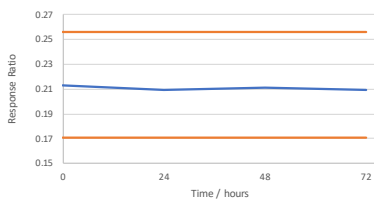

Low Concentration Acryl fentanyl

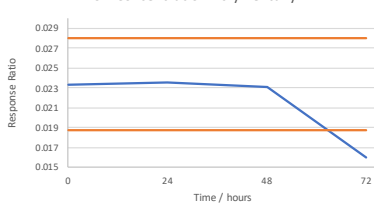

High Concentration Acryl fentany

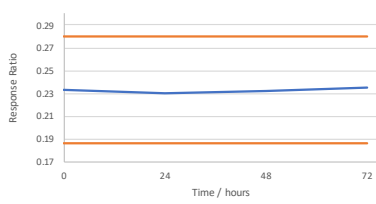

Low Concentration Carfentanil

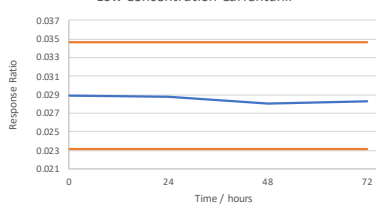

High Concentration Carfentanil

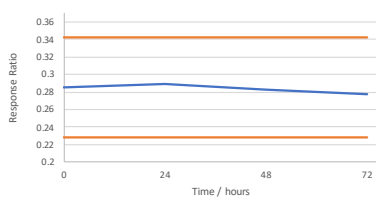

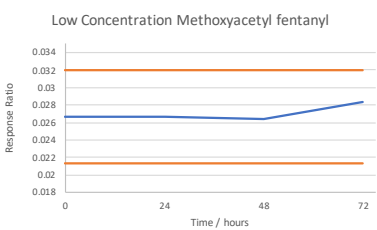

High Concentration Methoxyacetyl fentanyl

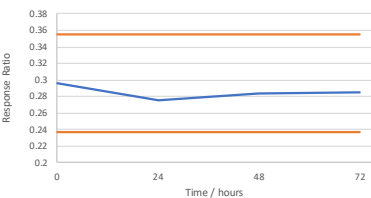

ow Concentration Furanyl fentanyl

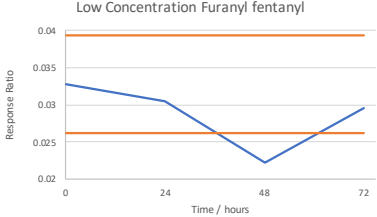

High Concentration Furanyl fentany

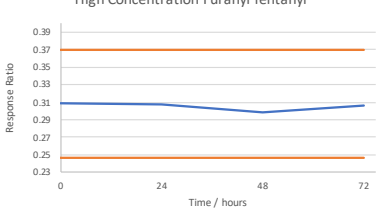

Low Concentration 2' fleoro ortho flvorentany

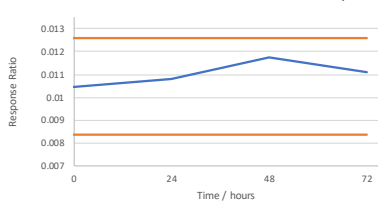

High Concentration 2'-fluoro ortho-fluoro
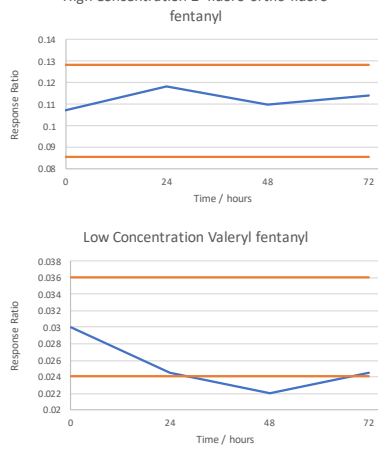

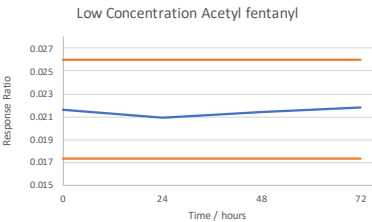

High Concentration Acetyl fentanyl

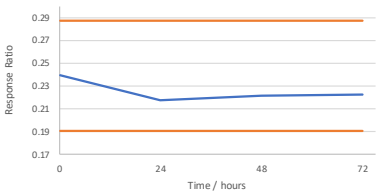

Low Concentration Despropionyl para-

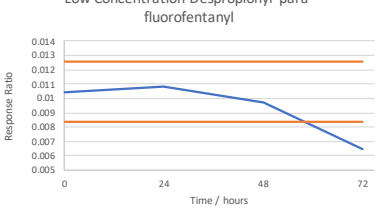

High Concentration Despropionyl para

fluorofentanyl

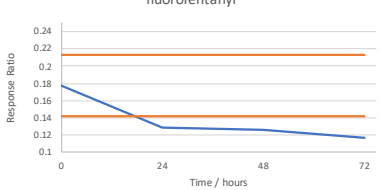

Low Concentration U-47700

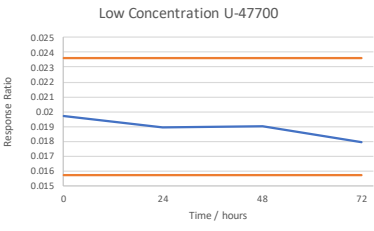

High Concentration U-47700

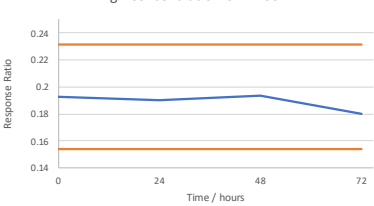

High Concentration Valeryl fentanyl

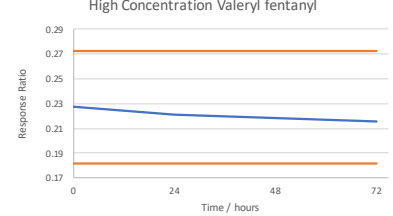

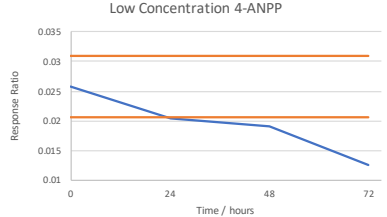

High Concentration 4-ANPP

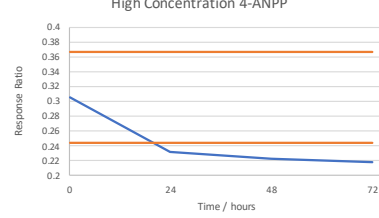

Low Concentration Fentany

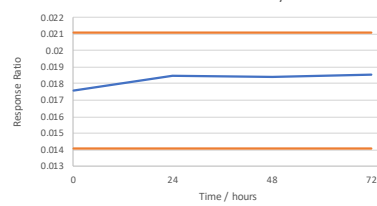

High Concentration Fentany

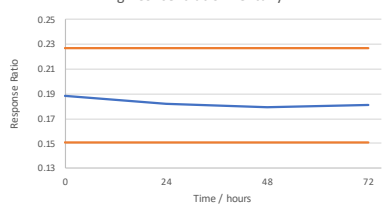

Low Concentration FIBF

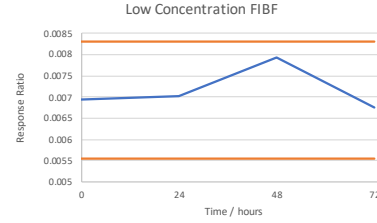

High Concentration FIBF

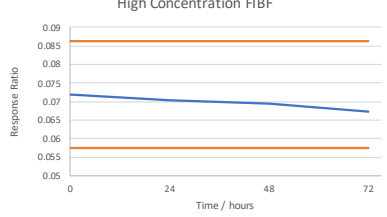

Figure A6: Plots demonstrating response ratio versus time, representing the stability of analytes at both the low and high concentrations over 72 hours at room temperature for autosampler processed sample stability. 

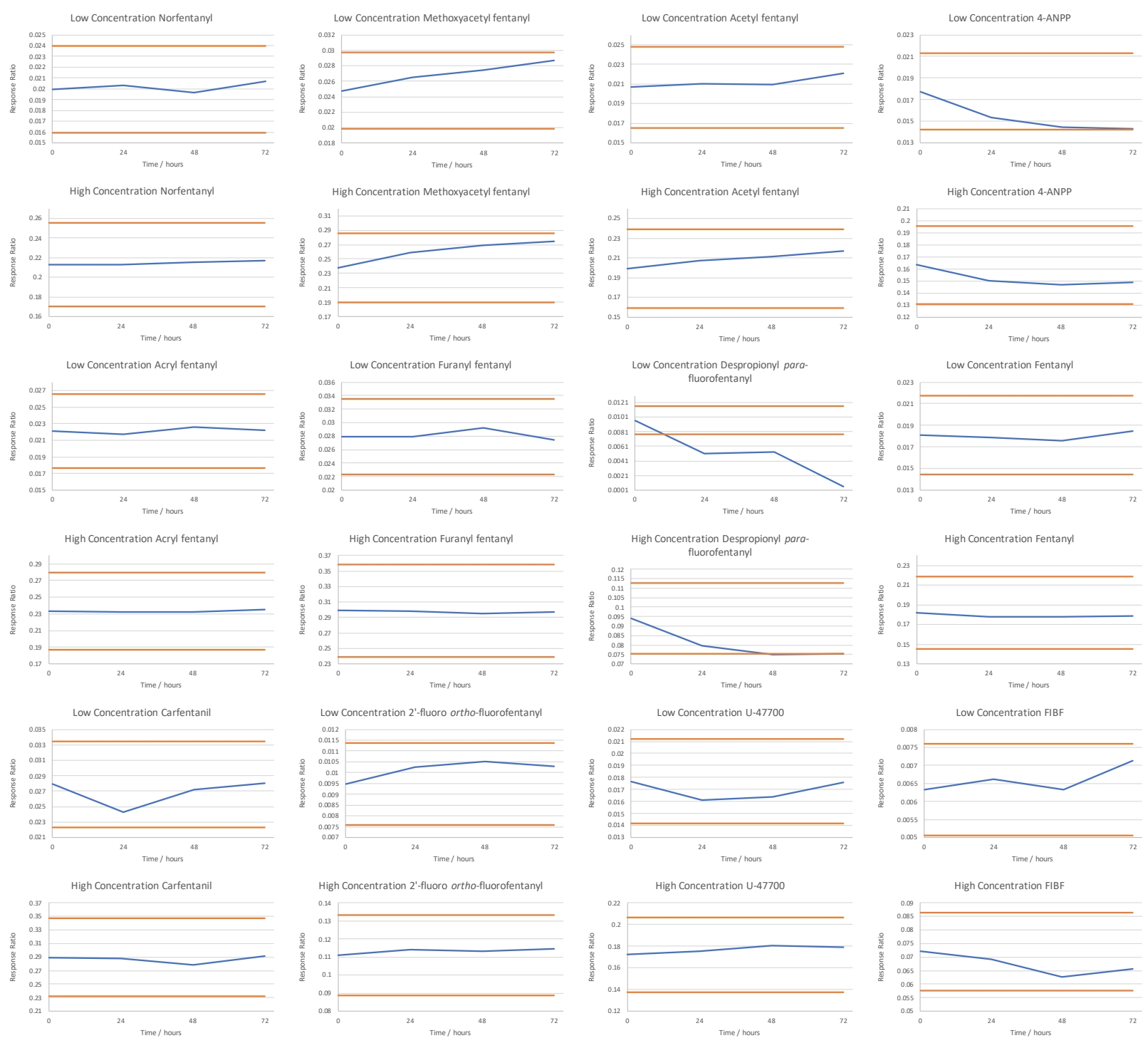

Figure A7: Plots demonstrating response ratio versus time, representing the stability of analytes at both the low and high concentrations over 72 hours at $-20^{\circ} \mathrm{C}$ for processed sample freezer stability.
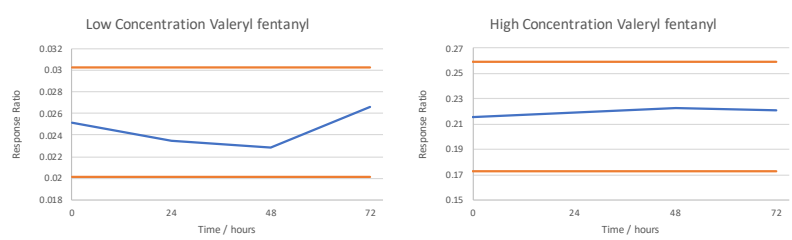